\title{
Mind the gap: collaboration between practice, policy and research in local public health
}

Citation for published version (APA):

Jansen, M. W. J. (2007). Mind the gap: collaboration between practice, policy and research in local public health. [Doctoral Thesis, Maastricht University]. Datawyse / Universitaire Pers Maastricht.

https://doi.org/10.26481/dis.20070628mj

Document status and date:

Published: 01/01/2007

DOI:

10.26481/dis.20070628mj

Document Version:

Publisher's PDF, also known as Version of record

\section{Please check the document version of this publication:}

- A submitted manuscript is the version of the article upon submission and before peer-review. There can be important differences between the submitted version and the official published version of record.

People interested in the research are advised to contact the author for the final version of the publication, or visit the DOI to the publisher's website.

- The final author version and the galley proof are versions of the publication after peer review.

- The final published version features the final layout of the paper including the volume, issue and page numbers.

Link to publication

\footnotetext{
General rights rights.

- You may freely distribute the URL identifying the publication in the public portal. please follow below link for the End User Agreement:

www.umlib.nl/taverne-license

Take down policy

If you believe that this document breaches copyright please contact us at:

repository@maastrichtuniversity.nl

providing details and we will investigate your claim.
}

Copyright and moral rights for the publications made accessible in the public portal are retained by the authors and/or other copyright owners and it is a condition of accessing publications that users recognise and abide by the legal requirements associated with these

- Users may download and print one copy of any publication from the public portal for the purpose of private study or research.

- You may not further distribute the material or use it for any profit-making activity or commercial gain

If the publication is distributed under the terms of Article $25 \mathrm{fa}$ of the Dutch Copyright Act, indicated by the "Taverne" license above, 


\section{Mind the gap: \\ Collaboration between practice, policy and research in local public health}

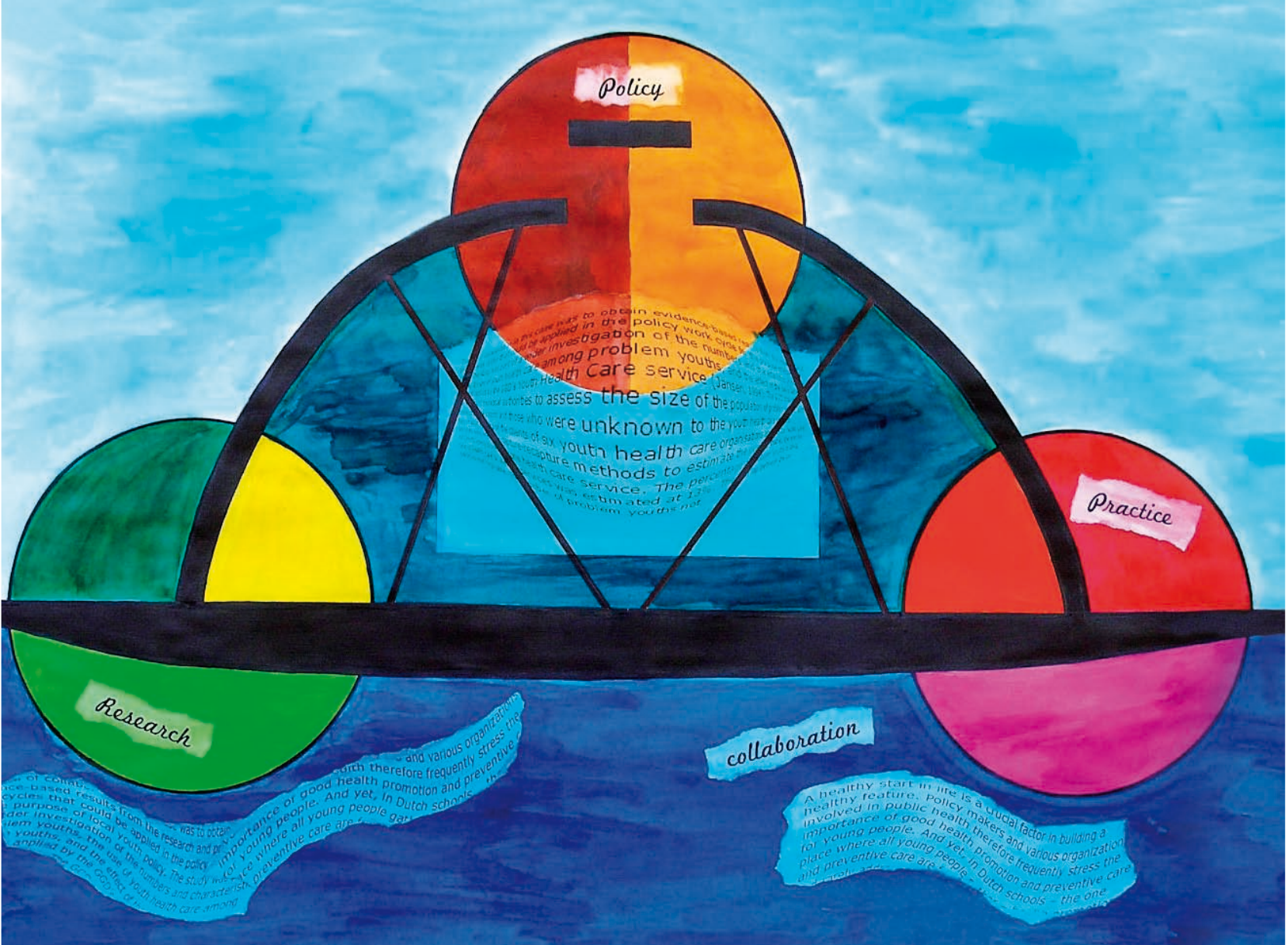

Maria Jansen 


$$
\begin{aligned}
& \text { Mind the gap: } \\
& \text { Collaboration between practice, } \\
& \text { policy and research in local public health }
\end{aligned}
$$


(C) M. W. J. Jansen, Maastricht 2007

Cover design: (C) Wouter J. van Dongen, Désirée M. van Dongen, Sophie I. van Dongen Layout: Wouter J. van Dongen

Production: Datawyse / Universitaire Pers Maastricht

\section{ISBN 978-90-5278-626-1}

All rights reserved. Illustrations and brief excerpts from this publication may be used for scientific and educational purpose provided that the source is acknowledged. 


\title{
Mind the gap: \\ Collaboration between practice, policy and research in local public health
}

\author{
PROEFSCHRIFT \\ ter verkrijging van de graad van doctor aan de Universiteit Maastricht, \\ op gezag van de Rector Magnificus, Prof. mr. G.P.M.F. Mols \\ volgens het besluit van het College van Decanen, \\ in het openbaar te verdedigen op donderdag 28 juni 2007 om 14.00 uur \\ door
}

Maria Wilhelmina Jacoba Jansen

$\underline{\underline{M}}$ 


\section{Promotores}

Prof. dr. N.K. de Vries

Prof. dr. G.J. Kok

Prof. dr. J.A.M. van Oers (Universiteit van Tilburg)

\section{Beoordelingscommissie}

Prof. dr. H.W. van den Borne (voorzitter)

Dr. P.T. van Assema

Prof. dr. T.E.D. van der Grinten (Erasmus Universiteit Rotterdam)

Dr. ir. J.M.M. Meijers

Prof. dr. C. Spreeuwenberg 
Ter nagedachtenis aan mijn vader 


\section{Contents}

$\begin{array}{ll}\text { Prologue } & 9\end{array}$

Chapter 1. Background and problem definition 11

Introduction 11

$\begin{array}{ll}\text { Public health } & 12\end{array}$

Public health policy $\quad 15$

Public health practice $\quad 18$

Public health research $\quad 19$

Problem statement 20

Research questions and design $\quad 25$

Multiple-case study 25

$\begin{array}{ll}\text { Outline of the thesis } & 27\end{array}$

Chapter 2. Policy, practice and research 31

Introduction 31

Policy cycle $\quad 32$

Practice cycle $\quad 37$

Research cycle 41

Which gaps need to be bridged? $\quad 45$

Summary $\quad 54$

Chapter 3. Determinants, theories and practical strategies 55

Introduction 55

The logic model $\quad 57$

Administrative level $\quad 58$

Institutional level $\quad 62$

Individual level $\quad 65$

$\begin{array}{ll}\text { Overview of practical strategies } & 67\end{array}$

$\begin{array}{ll}\text { Summary } & 70\end{array}$

$\begin{array}{ll}\text { Chapter 4. Expert meeting } & 71\end{array}$

Introduction $\quad 71$

Design and respondents $\quad 71$

Results $\quad 73$

$\begin{array}{ll}\text { Conclusion } & 75\end{array}$ 
$\begin{array}{ll}\text { Chapter 5. Multiple-case study } & 81\end{array}$

Outline 81

The cases $\quad 82$

Analysis 83

The risk of subjectivity $\quad 84$

5.1. Case 1. Introduction

Estimating the size of the population of problem youths in the Dutch town of Maastricht, using Frank-Snijders estimators

Case 1. Overview of applied practical strategies

5.2. Case 2. Introduction

Sexually transmitted diseases in the Dutch province of Limburg in 1997: prevalence figures based on a survey among general practitioners and specialists and on data from microbiology laboratories

Case 2. Overview of applied practical strategies

5.3. Case 3. Introduction

Effects of extended opening hours of gambling centres on problem gambling Case 3. Overview of applied practical strategies

5.4. Case 4. Introduction

Systematic approach to hepatitis B vaccination programmes for risk groups: a literature study

Case 4. Overview of applied practical strategies

5.5. Case 5. Introduction

The tailored approach of the 'Schoolbeat' programme: new concepts for health promotion in schools in the Netherlands Case 5. Overview of applied practical strategies

5.6. Case 6. Introduction

Continuation processes for a successful preventive intervention: Heartbeat 2. The concept of sustainability and the use of outcome indicators

Case 6. Overview of applied practical strategies

Generalizing the findings of the multiple-case study

Results

Overall concluding remarks 
Chapter 6. General discussion and future prospects

Introduction

General conclusions

Strengths and limitations of this study

234

Discussion and recommendations

Future prospects

References

Summary

Samenvatting

Dankwoord

Curriculum vitae 


\section{Prologue}

The Regional Public Health Service (GGD) is an important player in the field of public health. Commissioned by the local government, the GGD implements various tasks to protect and promote public health. I have been working with the GGD since 1989, which means that I have eighteen years of experience in this field. During this period, I was confronted with persistent problems concerning the collaboration between the three domains of public health policy, practice and research - a collaboration which I deemed necessary to achieve effective public health. I became more and more fascinated by the question why this collaboration was so difficult to achieve and why it so often failed. In the end, this question became the starting point for this dissertation.

Problems dealt with in the field of public health are highly diverse. For instance, the problems I have addressed over the last eighteen years have included estimating the size of a population of problem youths; assessing the reliability of the registration of sexually transmitted diseases; advising on local policy measures to reduce problem gambling; developing a hepatitis B vaccination campaign to reach hidden risk groups; reorganizing the supply of services to schools to improve healthy school policy, and developing a prevention programme to reduce cardiovascular diseases. This dissertation is based on an analysis of these six cases. Each case concerns a research question about a public health problem with practical and policy implications. I have published or submitted scientific articles about these six public health cases, which are also included in this dissertation. These articles were intended to communicate the empirical findings on the object of study. These examples are also used in the dissertation for an examination of the question why it is so difficult to coordinate public health policy, practice and research. The cases illustrate the weak and strong points of collaboration between practice, policy and research within public health, that is, what goes wrong and why, but also what goes right and why.

The articles are presented in the sequence in which they were written, and show a certain development over time, which can be traced back to my own professional career and the professional developments that took place at the GGD. I started work as a health promoter in 1989, after which I became coordinator of AIDS and addiction prevention, manager of a division of the GGD, institutional innovator, and finally programme manager of the Academic Collaborative Centre of Public Health in Limburg.

The central question in this dissertation, concerning the collaboration between practice, policy and research in local public health, was addressed as fol- 


\section{PROLOGUE}

lows. I first developed a theoretical model to explain the poor collaboration, after which I studied the relevant theory to identify options to improve the collaboration, which resulted in a series of change options. I then used the six specific public health examples as cases and reflected on my eighteen years of experience. Since the articles as such gave little or no information on the issues raised in the general research question, I supplemented the information by means of data from other sources. In this way I tried to find an answer to my question about the intractable problem of collaboration between public health practice, policy and research. 


\section{Background and problem definition}

\section{INTRODUCTION}

Public health has often been defined in terms of its aim and goals rather than by referring to any specific body of knowledge ${ }^{1-4}$. Public health includes: (i) policy, as it is inherently a political enterprise; (ii) practice, as policies need to be implemented to create social action and to organize service delivery and (iii) research, to assess the prevalence and determinants of health problems and the effectiveness and cost-benefit ratio of implemented activities. Public health goals can only be achieved if many different disciplines contribute, including social epidemiology, biostatistics, behavioural science, biomedicine, genetics, nutrition, psychology, social science, social administration, public policy, organizational development, innovation policy, strategic management and social marketing.

Ideally, policy, practice and research should be collaborative and mutually dependent partners, uniting many different disciplines. In reality, however, the field of public health lacks sufficient connections between academic research, practice and policy. The three domains do not easily converge, despite universal calls for collaboration in order to support public health. In the early twenty-first century, the need to strengthen public health by linking public health policy with research and practice is becoming increasingly apparent, giving rise to calls for (i) creating synergism, (ii) generating sufficient critical mass, (iii) increasing public health visibility and (iv) achieving the necessary multidisciplinarity to obtain more solid evidence ${ }^{5-25}$.

This dissertation focuses on the question what barriers are preventing an interlinked development and how these barriers can be overcome. It explores the reasons for the lack of coherence between public health policy, practice and research in the Netherlands. It argues that the lack of connections can be traced back to ambiguous and conflicting goals and expectations, having their origin in the niche character of the component areas of public health. Each area operates independently and has its own professional standards, frame of reference, organizational structure, formal and informal networks ${ }^{26-30}$. The dissertation proposes a model for initiating and sustaining collaboration. This introductory 


\section{CHAPTER 1}

chapter starts by discussing the main concepts of policy, practice and research, followed by an outline of the problem statement, the research questions and the study design.

\section{PUBLIC HEALTH}

Public health is the process of mobilizing and engaging local, national and international resources to ensure conditions in which people can be healthy. Since its early days, public health has been conceived in terms of three relatively distinct missions, which have more or less succeeded each other in time: (i) public health as a reaction to epidemics, (ii) public health as a form of restrictive policy to regulate communal life and (iii) public health as a means to improve quality of life, sometimes referred to as 'betterment" ${ }^{31}$. In other words, the mission of public health has consecutively been characterized by reactive, regulatory and proactive approaches. The first phase evolved during the eighteenth and nineteenth centuries. It is known as the sanitary movement, prompted by the epidemiological studies made by Snow and Budd, showing the water-mediated transmission of cholera and typhoid. The bacteriological revolution, started by the work of Louis Pasteur and Robert Koch, provided enormous benefits to medicine and public health through vaccination and immunization campaigns. Regulatory standards on housing and environmental conditions as well as water supply were introduced, giving rise to the second phase of public health development, from the late nineteenth through the mid-twentieth century, referred to here as restrictive policy. Restrictive policy refers to various actions taken at all levels of government to enforce regulations for the common good. This policy included aspects like regulating hours and conditions of work, investigating forensic matters, instigating rules on burial, on the treatment of cattle, rules on travellers and prostitutes, and caring for the poor. The recognition of the health effects of nutrition, inspired by the work of pioneers such as James Lind and Joseph Goldberger, was an important milestone in the further development of public health. In the first half of the twentieth century, food and labelling standards, food fortification and food supply to the poor were promoted and rules for market places and the quality of commodities were enforced. Preventive care for women and children became a public health issue and maternal and child health services were set up. As diseases caused by infectious agents and vitamin shortages were brought under control, cardiovascular diseases, trauma and cancer became the predominant causes of death in the $1960 \mathrm{~s}^{3}$. Reliable mortality and morbidity statistics became available as a part of modern public health, providing a better understanding of the avoidable and remediable causes of disease. For instance, the relationship between tobacco use and lung cancer was demonstrated from the geographic variation in cancer incidence ${ }^{32,33}$, 


\section{BACKGROUND AND PROBLEM DEFINITION}

and other lifestyle factors also became the focus of scientific investigations. Industrialized countries witnessed a major shift in epidemiological patterns from infectious to non-infectious diseases. This epidemiological shift made it clear that lifestyle determinants have a major impact on the occurrence of highly prevalent chronic diseases like cardiovascular diseases and cancer ${ }^{3,31}$.

The recognition of differential mortality and morbidity patterns led to the most recent phase in the development of public health. Its new mission is not merely reactive or regulatory, but proactive, trying to reduce preventable or avoidable mortality and morbidity and to improve people's quality of life $\mathrm{e}^{3,31,34}$. In this respect, the Lalonde report was a landmark publication, proposing a comprehensive policy on avoidable mortality and morbidity. The WHO has established a long-term programme called 'Health For All by the year 2000' (HFA 2000), specifically to tackle issues pertaining to inequities in health and healthy life expectancy. The concept of HFA 2000 refers to a wider framework, which is also defined as the New Public Health, marking a shift away from the biomedical emphasis on the individual towards a focus on social and environmental factors in the aetiology of health problems. This wider framework encompasses all modalities, including clinical and prevention-oriented services, to effectively and economically preserve, protect and promote the health of the individual and of society. It also considers ethical issues which relate to health expenditure, priorities and social philosophy $3,35,36$. The Health For All programme is aiming at the attainment of health at a level that should permit all citizens to lead a socially and economically productive life. The first leading principle for HFA 2000 was expressed at the 1978 WHO conference in AlmaAta, and focused on primary health care, dealing not only with health care as such but also with its social and economic context. Some years later, the industrialized welfare states called for a programme to tackle health inequities, beyond the ideology of primary health care. This resulted in the second leading principle for HFA 2000, relating to health promotion, which was formulated at the WHO conference at Ottawa in 1986. Health promotion has become an indispensable aspect of public health since the Ottawa Charter, aiming to address lifestyle determinants, which are more complex than the determinants of vector-born infectious diseases (i.e., those transmitted via intermediate hosts), in terms of causation and means of prevention. Health promotion is defined as the process of enabling people to increase their control over, and to improve, their own health ${ }^{37}$.

The Ottawa Charter defined five domains of health promotion: building healthy public policies, creating supportive environments, strengthening community action, developing personal skills, and re-orienting health services. Health issues are often beyond the capacity of individuals or communities and require a healthy environment and a healthy public policy to facilitate healthy behaviour ${ }^{38-43}$. The Netherlands has acted upon the HFA 2000 commitment, 


\section{CHAPTER 1}

and the Dutch government published a memorandum called Nota 2000 in 1986, proposing a future-oriented, strategic health policy. Unfortunately, the memorandum was evaluated as having limited feasibility ${ }^{44}$. Nowadays, health promotion at the individual, group and community levels, implemented in various settings like family, school, community, work and clinical medical care, is gradually becoming common practice in the Netherlands.

The proactive public health mission was reaffirmed in the general accepted definition proposed by Donald Acheson, a senior professor of public health in the United Kingdom. In view of the increasing social disparities, he specified public health as 'the science and art of preventing disease, prolonging life and promoting health through the organized efforts of society'. Nowadays, health is not merely the absence of disease but is regarded as a state of physical, social and mental well-being, with special focus on the quality of life, as is expressed in the World Health Organization's definition. Achieving this goal requires a broad scope of strategies, and assessment of their effectiveness ${ }^{45,46}$.

The organization and management of the public health system affects the health and well-being of individuals and populations ${ }^{47}$. Progress in public health is facilitated by supplying services and allocating resources. Resource allocation is sensitive to economic and political factors and impacts on the priorities in public health policy. The agenda of public health policy is becoming dominated by issues associated with the aging and increasing longevity of the population, social health inequalities, ecological and environmental imbalances, unhealthy lifestyles in affluent societies, and sustainable resources. These issues fit in with the current mission of betterment, and imply major redistributions in power and wealth ${ }^{36,48}$. The term public health, as it is used in this thesis, should be interpreted in the broader sense, involving personal, environmental, economic and political aspects.

As stated above, public health has been defined in terms of its aims and goals, rather than by any specific discipline or body of knowledge. Mission, goals and methods have changed over time. The transition from the reactive to the regulatory approach gradually arose with the growing consciousness that public health practice, as it had emerged from medical science, needed formal policy to become embedded in society. Linking research and practice to policy and vice versa proved not to be an easy process, and in the course of history, many public health issues were heatedly debated before practical applications and policy reforms were achieved ${ }^{49}$. For instance, the time that elapsed between the initial evidence that citrus fruits prevented scurvy and the adoption of a universal preventive policy within the British navy was 264 years ${ }^{50}$. Various cholera epidemics passed, killing thousands of people, before policy guidelines were accepted. Those who believed in water-mediated cholera outbreaks were opposed by powerful forces, before water filtration legislation became common practice. With the transition from the regulatory to the proactive approach, the 


\section{BACKGROUND AND PROBLEM DEFINITION}

public health field came to realize the complexity of the evidence. So far, many discoveries have resulted from serendipity, followed by scientific research under carefully controlled conditions in order to prove their general validity. The evidence about proactive actions for the betterment of people, however, inevitably raises ethical, technical, practical, political, organizational and economic issues, making the evidence extremely complex ${ }^{20,50-53}$. In view of the substantial potential offered by preventive action and health promotion, the need for evidence is obvious. This concerns research-based evidence, evidence-based practice as well as evidence-based policy. For example, the scientific evidence about the relation between physical inactivity and obesity is not sufficient to induce policymakers and practitioners to work on a series of feasible policy and practice options. As with many public health problems, this example clearly shows the need for long-term collaboration, because clear-cut technical solutions are often not acceptable and policy options within the public arena have to await a suitable political climate $20,50-56$. For instance, the public acceptability of a policy on passive smoking was shaped by many different factors, only one of which was the scientific evidence, and it took many decades to effectuate local, national and European policies on tobacco ${ }^{57}$. More than ever, the complexity of evidence stresses the need for sustained collaboration between practice, policy and research.

\section{PUBLIC HEALTH POLICY}

Public health policy concerns those publicly organized activities for which individuals or the market cannot or will not take responsibility and which relate to health issues at a collective or individual level. It is not based on individual health care requests as in cure and care. Public health policy must be founded in law, public administration and financing in order to meet the preconditions for healthy living. In the Netherlands, national and local governments, and for some aspects also provincial governments, have separate responsibilities for public health ${ }^{58}$. The legislation consists of a range of laws, dealing with collective prevention, medical care after accidents and disasters, emergency care, quality of commodities, infectious diseases, tobacco, screening and early detection, alcohol, catering, welfare, and quality assurance of preventive care. The Dutch Health Care Inspectorate is the official monitoring institute, which reports its findings to the Ministry of Health, Welfare and Sport.

Collective prevention, being part of public health policy, was the responsibility of the Minister of Health, Welfare and Sport until 1989, when it was decentralized to local authorities as stipulated by the Dutch Public Health Act (in Dutch: Wet Collectieve Preventie Volksgezondheid, WCPV). This law determines the basic statutory duties on collective prevention in global terms. From 


\section{CHAPTER 1}

1989 onwards, local authorities, i.e., the Regional Public Health Services (referred to below as GGDs) have been invited to implement detailed programmes tailored to the local setting. Decentralization was deemed to be necessary to reduce the distance between the authorities and civilians and to make services fit in more closely with civilians' needs. Under the WCPV, the municipal authorities became responsible for the following tasks: attuning prevention to curative medicine; epidemiological assessment of the health status of the population; monitoring health aspects of administrative decisions; health promotion and health education; environmental health care; technical hygiene to control microbial threats; public mental health care including a safety net for vulnerable people and refugees; surveillance and control of infectious disease including aids, sexually transmitted diseases and tuberculosis; and preventive youth health care ${ }^{59,60}$.

The decentralization process was monitored by the Inspectorate, which reported extremely disappointing results in 1995. This in turn resulted in the installation of a national steering committee for the reinforcement of public health (commissie Lemstra). Policy recommendations for the Minister made by this committee included a review of the law on collective prevention and the establishment of a Platform for Public Health Care in order to put public health on the agenda at the Ministry of Health, Welfare and Sport, the Ministry of the Interior, the Association of Municipalities in the Netherlands, and the national association of GGDs ${ }^{61,62}$. The Platform had to improve and financially facilitate public health practice with a one-off budget of 18 million euros for the 20022006 period. The WCPV was amended in 2003, leading to the obligation to issue a public health policy memorandum every four years, as an impetus to priority setting and programme implementation, both at the national and the local level ${ }^{63}$. The national policy programme was given high prominence by the Ministry of Health, Welfare and Sport because the Netherlands was being confronted with a decline in healthy life expectancy compared to other European countries, after being at the top of the league table for many years ${ }^{64-73}$. The national policy programme provides a framework for municipal policy programmes.

The 2003 amendment to the WCPV led to a new category of relatively inexperienced public health stakeholders at the local level, which partly replaced the GGDs as primary stakeholders, and took over responsibilities from the GGDs. These stakeholders were public administrators, civil servants and aldermen responsible for public health, all of them having many other responsibilities besides public health. Their collaboration with the GGDs varied per municipality, depending on the advisory tasks municipal authorities allocated to the GGD. The Association of Municipalities in the Netherlands (VNG) recommended that local policy makers should use evidence-based public health programmes, implicitly assuming that local stakeholders had sufficient knowl- 


\section{BACKGROUND AND PROBLEM DEFINITION}

edge of the evidence on public health ${ }^{74,75}$. In 2005 , however, the VNG concluded that civil servants were insufficiently equipped in terms of both available time and capacity to accomplish the task of drafting local public health policy ${ }^{76}$.

Just as in 1995, local governments were once again confronted with unfavourable evaluation results in 2005. The Dutch Health Care Inspectorate concluded that the evidence, the quality and the implementation of local public health policy were unsatisfactory ${ }^{77}$. The criticism was clear: the new explicit role of the municipal authorities in defining public health policy had improved but not yet attained its full capacity. Furthermore, public health policy was still insufficiently prepared for emerging health problems such as obesity, large-scale epidemics, psycho-social problems among young people and acute care for those with serious mental disorders jeopardizing public safety. The central role in providing public health services was not yet being sufficiently guided by local public health policy. About half of the municipal health departments had not even set up a systematic evaluation of programmes and policy. Based on these results, the Inspectorate recommended to the Ministry to establish a national health promotion centre, to supervise and support municipalities on local public health. In response, the Ministry has taken steps to set up centres of expertise at the National Institute of Public Health and the Environment (RIVM) to support local policy on infectious disease, youth health care, screening and health promotion ${ }^{78}$. Once again, the Inspectorate is emphasizing the need for quality improvement in public health, without commenting on budgets, either national or local. Public health policy and its implementation are largely funded by the local governments. Nationally, $1 \%$ of the annual health care budget is invested in prevention and public health ${ }^{79}$.

An overview of the domain of Dutch public health policy shows that its weaknesses result from a continuous struggle to determine the scale of the regions for which policies are to be implemented, the corresponding mandatory power and the available resources. Although decentralized implementation, tailored to the people whom it concerns, is a positive starting point, experience since 1989 has shown that decentralized responsibility has not resulted in the desired reinforcement of public health policy at the local level, neither within the proximal policy areas of public health and welfare nor in the more distant policy areas such as economic and social affairs. Local authorities lack the time and competence to investigate the minimum requirements for local public health policy ${ }^{80}$. Moreover, several public health experts in the Netherlands and abroad have recognized the difficulties of influencing policymakers to actually give priority to public health issues and to make policies health-sensitive. $\mathrm{Na}$ tionally assessed directives, based on minimum requirements for effective local public health policy, may provide an impetus to local policy makers to put public health on the policy agendas of different policy departments to promote a comprehensive and integrated view. An integrated view covers the desired pub- 


\section{CHAPTER 1}

lic health goals for all policy areas (in Dutch: facetbeleid) in order to make local policies health-sensitive ${ }^{6,49}$. What is needed is a set of instruments to support intersectoral decision-making across the different policy areas, such as the Health Impact Assessment (HIA) and the Environmental Impact Assessment (EIA) instruments ${ }^{80-85}$. The HIA and EIA offer a combination of procedures, methods and tools by which a policy or a programme may be judged as to its potential effects on the health of a population, and the distribution of these effects within the population ${ }^{86}$. If these instruments are developed further, their systematic application may help sensitize policy-makers to the public health effects of policies ${ }^{87,88}$.

The magnitude and success of public health efforts unequivocally depend on the resources available ${ }^{89}$. Until 1989, national mandatory guidelines about minimum local investments per inhabitant were part of public health policy and these quantified budgets were made available to local authorities. The reintroduction of such public health investments quantified per inhabitant may be considered, regardless of whether the origin of the budget is national or local. This origin, it must be realized, is a political debate.

\section{PUBLIC HEALTH PRACTICE}

Public health practice is the primary domain of the GGDs, which co-operate with a broad range of agencies (private as well as public) such as welfare, education, housing, police, health care, mental health care, child health care, traffic safety, etc. It is especially since the development of its proactive mission that public health practice requires intersectoral collaboration, since most public health problems are multi-causal in nature.

Public health practice by GGDs started in the nineteenth century and expanded only slowly until the 1960s and $1970 \mathrm{~s}^{90,91}$. This practice has acquired a more mature status since the 1970s, resulting in a comprehensive national network of GGDs by 199092. The Public Health Act (WCPV) obliges all municipalities to establish and maintain a GGD. GGDs are the agencies implementing public health policy, which have to ensure that municipal authorities operate according to national public health policies and directives 93,94 . Since the introduction in 2003 of the mandatory obligation to write local public health policy memorandums, GGDs have been compelled to turn their rather closed professional policy into collaborations with the civil service in order to advise on local public health programmes. Based on the WCPV, GGDs need to employ professional staff with expertise in social medicine, health promotion, epidemiology, social nursing, dental care, behavioural science and information science. These professionals are the main public health practitioners. In retrospect, the practical contribution to public health started with the contributions made by 


\section{BACKGROUND AND PROBLEM DEFINITION}

professionals from social medicine (occupational medicine, youth health care, public health care) and social nursing, followed by epidemiologists and health promoters. In 2000, the Inspectorate concluded that the quality of health promotion practice was below professional standards ${ }^{95}$. A task force for the reevaluation of health promotion implementation (in Dutch: Task Force Herijking Gezondheidsbevordering-functies) was installed at the request of the Ministry. The Task Force has made recommendations for the improvement of the professional standards of health promoters ${ }^{96}$.

Local authorities are the principal financiers of the GGDs. To give an impression of the magnitude of current budgets, the GGD for the Southern Limburg region is employing a staff of 0.6 full-time equivalents (fte) on active service per 1000 inhabitants (2006 figures). The annual budget requested for 2006 amounts to 17 euros per inhabitant, including all tasks under the WCPV and acute medical care for accidents and disasters, while the national mean is 23.50 euros per inhabitant $\mathrm{t}^{97,98}$. The relationship between local authorities and GGDs is often characterized as ambivalent, because the local authorities both own the GGD in a financial sense and make the decisions on local public health policy. As proposals or regulations have budgetary consequences which might result in unpredictable public commotion in the political arena, local policy makers are extremely reticent, and keep a certain distance from practice.

Occupational health, although an important field within public health, is beyond the scope of this dissertation (as well as that of the legal and operational framework of the WCPV). After World War II, occupational health became an important discipline within the GGDs' public health services. Since 1998, however, specialized private agencies (called Arbo-diensten in Dutch) took over the responsibility for worker health, as a result of amendments to national legislation and changes in financial responsibilities. Employers became responsible for the workplace environment and occupational safety of their employees. Since that time, policy and organizational development in occupational health care have come to differ greatly from collective prevention and emergency care after disasters, for which the GGDs bear the statutory responsibility. Occupational health is no longer a core business of the GGDs and was therefore excluded from the current research project.

\section{PUBLIC HEALTH RESEARCH}

Public health research is the domain of universities and research institutes. According to a 2003 survey by the Dutch Advisory Council on Health Research, universities provided $37 \%$ of the total public health research, research institutes $55 \%$, and GGDs $8 \%{ }^{28}$. The total public health research volume is estimated to be 730 full-time equivalents, 272 of which are located at universities, corre- 


\section{CHAPTER 1}

sponding to about $4 \%$ of the total university health research capacity. University research is rather fragmented in the Netherlands. Some universities have departments for social or community medicine, while other universities have focused on the development of health promotion, health policy, health economics or epidemiology. The main non-university research institutes contributing to public health research are the National Institute of Public Health and the Environment (RIVM), the Netherlands Organisation for Applied Scientific Research (TNO), the Dutch Institute for Health Care Research (Nivel), the National Institute on Health Promotion and Disease Prevention (NIGZ), the Consumer Safety Institute (Stichting Consument en Veiligheid), and the Netherlands Institute on Mental Health and Addiction (Trimbos-instituut). Public health research as implemented by GGDs is mainly limited to monitoring the health status of the population. The spread of research over a large number of groups means that the potential for synergy is not used to the best advantage.

The major academic disciplines involved in public health research are social medicine, epidemiology, health promotion and health organization, together with related clinical and laboratory disciplines. However, from the 1970s onwards, the vitality of the academic discipline of social medicine has declined $^{99}$. Since social physicians were in practice made responsible for an increased volume of routine check-ups there was an influx of less innovative and more routine-based social physicians, without research aspirations. The decreased academic vitality and the increased routine tasks have reinforced each other, further widening the gap between practice and research ${ }^{17,100}$. Furthermore, the increasing competition by epidemiologists, health promoters and experts on health economics and organization, who are supposed to contribute to public health research from a multidisciplinary point of view, has caused a further decline in the professional status of social medicine.

The Advisory Council on Health Research concludes that the knowledgeinfrastructure, i.e. "the comprehensive structural provisions aimed at generating and applying high quality expertise to public health practice", is unsatisfactory ${ }^{28}$.

\section{PROBLEM STATEMENT}

The evidence that is necessary to attain public health goals can only be generated by the combined contributions of research, practice and policy. Although evidence-based practice and evidence-based policy have become extremely popular concepts within the field of public health research $5,20,50,101-108$, the rhetoric has so far largely exceeded reality. 


\section{Niches and pace}

Public health development is to be conceived as emanating from three more or less independent 'niches'. The term niche stems from biology and stands for the whole complex of environmental conditions necessary to maintain a particular species. In the sense in which the term is used here, niches are characterized by specific ideologies, values and norms, internal orientation, specific communication and niche language, independence, nternal codes of behaviour, and self-directed improvement processes. They have dynamics of their own, with a strong desire to protect themselves against the outside world. These characteristics also apply to the niches of policy, practice and research. Each niche has its own culture expressed in a mission, goals, strategies, organizational climate, interpersonal relations, professional standards, criteria for evidence, hierarchy, formal and informal networks, working methods, communication structure, language and accountability 5,27,29,57,109.

The niche character can be explained from the constructivist approach to networks ${ }^{110}$. This approach implies that people construct their own reality depending on the network - or niche - in which they work and live, giving meaning to their lives in a complex world. Each niche creates its own social and cognitive dimension, including rules with regard to language, procedures, habits, interpersonal contacts, informal behaviour etc. This results in a frame of reference which can only be observed by the actors within the niche, since it is not possible to perceive that which is unknown and beyond one's own world. The niche is closed to outside actors, which is the effect of the frame of reference. Actors within the niche reinforce the frame of reference because acting in this way gives meaning to their lives, whereas critical appraisal of one's own frame of reference by others is considered a threat, making innovations or changes extremely difficult. Proposals to familiarize oneself with other frames of reference in order to judge one's own usually meet with negative reactions, and judgements about the other niches are often no more than prejudices.

A further complication is the difference in work pace between the niches: it is extremely difficult to keep each process in pace with the often unpredictable needs of the others. Both of these gaps, in pace and in niche characteristics, seem to be widening because of the current emphasis on academic rigour and abstraction. Some authors have even stated that the differences are so great that no overlap exists ${ }^{27,49,111}$.

\section{Urgent need for evidence}

Although the 2003 amendment to the law (WCPV) has meant that policy and practice are forced to collaborate, the new nature of this relationship is not 


\section{CHAPTER 1}

without conflicts ${ }^{112}$. Although the niche members individually and independently express the need to develop public health progress in relation to risk patterns and diseases of modern life, through concerted actions by policy, practice and research, the unfavourable judgments by the Inspectorate on municipal public health policy in 1995 and 2005 demonstrate that such concerted action is still rare. Over the last decades, the research domain has developed many evidence-based interventions, but their practical implementation and the attention devoted to policy implications have lagged far behind. The Advisory Council on Health Research has recommended a concentration of research, a more solid academic foundation for public health policy and practice, and the establishment and maintenance of so called Academic Collaborative Centres for Public Health (called Academische Werkplaats in Dutch) to improve links between services, training and research 28,113 . The assumption that collaboration between practice, research and policy will result in more solid evidence and higher quality standards in public health is widely supported ${ }^{11,17,20,30,51,52,113-125}$. In spite of the perceived urgent need for evidence, many professionals have experienced how difficult it is to start and maintain a process of utilizing each other's expertise and to create an interface between the niches. Until now, thoughts about ways of shaping an interface between policy, practice and research have received relatively little attention. How to initiate and sustain collaboration is therefore the central issue of this dissertation.

\section{Causes of the persistence of niches}

There are various reasons for the persistence of the niche phenomenon and also of the problem of pacing.

Firstly, until 2005, the Ministry of Health, Welfare and Sport did not formally regulate collaboration between public health research, policy and practice, a necessary prerequisite for the advancement of Dutch public health policy. Recently, however, the Ministry has followed the recommendation of the Advisory Council on Health Research by providing extra resources for research and innovation over the next four years (2005-2010). This measure intends to improve cooperation between science, practice, policy and (postgraduate) education, which should ultimately lead to accessible, high-quality products, services and facilities in the field of public health ${ }^{126}$. Centres of expertise for public health will be initiated, in imitation of the existing centres of expertise for general practitioners, public mental health and rehabilitation medicine. Since this was the subject of recently formulated national policy, this aspect will not be further discussed in the present thesis. The effect of the ministerial measure will be evaluated at the national level, and the results are expected in 2011.

Secondly, existing organizations lack a formal communication structure to bridge the gaps between the niches. Although communication is sometimes 
facilitated by interpersonal contacts or the exchange of documents, multidisciplinary working groups are only formed in exceptional cases. Researchers, policy makers and practitioners do not have easy access to publications, databases, seminars, meetings, libraries etc. from the other niches, nor do they submit articles outside their own niche. There seems to be no urgently perceived need among policy makers, researchers and practitioners to organize direct communication lines themselves, not even on an informal basis. Although there have been some exceptions to this pattern, even these have their limitations. For example, a long-standing productive collaboration has developed in the Southern Limburg region between the local GGD, Maastricht University (UM) and the Maastricht University Hospital (azM). So far, however, this collaboration has often taken the form of temporary projects, which depend on co-incidental interpersonal relations. It mostly serves domain-specific goals, and is not consistently applied in all relevant public health areas. And sometimes it does not even yield simultaneous benefits to all partners. Other examples of collaborations between GGDs and universities include those between the Rotterdam Erasmus University, the Academic Medical Center at the University of Amsterdam, Tilburg University, the VU University Medical Center, Groningen University and the corresponding regional or municipal GGDs. To meet local public health policy objectives, the GGDs' practical purposes and the academic goals for research and teaching, the scope and effectiveness of these collaborations need to be further optimized.

Thirdly, the professionals involved in each niche are not sufficiently aware of the major differences between the niches with regard to values, ideologies, professional norms and goals of each niche. Policy makers are involved in processes of negotiation, bargaining and adjustment between different interest groups, in which formal and informal power, political salience, legitimacy, feasibility, public acceptability, public visibility, personal immediacy and public support play important roles. Their goal is to decide about the levels and allocation of resources in a specified period to achieve certain outcomes ${ }^{127-129}$. Researchers try to give rational and logical interpretations for real-life phenomena and act as 'dispassionate' creators of knowledge. Their goal is to add theories to the existing body of knowledge and to understand and explain specific phenomena ${ }^{130}$. Finally, practitioners intend to tailor their performance to the needs of their clients or community by improving their practices. Their goal is to contribute to solving the problems of individuals or groups and to improve the quality of decision-making in public health care ${ }^{59,60,93}$. These dissimilarities in professional norms result in differences in work pace between the niches, which impede interconnections. Practitioners often experience a sense of urgency, because people are in need, or an organization's performance remains below expectations. Practitioners expect immediate solutions from researchers, whereas researchers need thorough and lengthy investigations. Practitioners 


\section{CHAPTER 1}

then become restless and accuse researchers of delivering products that are far removed from reality and have no practical value. However, research demands high standards of reliability and validity of results and is therefore timeconsuming. Researchers accuse policy makers of pandering to political expediency, resulting in irrational outcomes, and of being complacent about the lack of evidence of many programmes used in practice. Since policy making is sometimes carried out under public pressure or pressure of time, especially in the case of crises or incidents, policy makers blame the researchers for delays in the process of decision- and policy-making, caused by evidence not being available. They want universities to teach students how to deal with policy matters and to train them in negotiation skills, while according to researchers even the time available to train them in academic skills is insufficient. The very high degree of disparities between the niches thus effectively keeps the three niches apart.

Fourthly, for professional and efficiency reasons, some parts of the work cycle in each niche are discussed and implemented with niche members only. This is no problem, provided that crucial points for the indispensable bridging of the gaps between the niches are recognized. These crucial points refer to steps marked within each work cycle, where shared commitment, concerted action or joint decision-making by all representatives of the policy, practice and research niches is essential. These crucial points are not always appropriately understood, although this is a prerequisite for sustained collaboration.

Fifthly, the existing differences between the niches lead to many barriers that prevent people from looking beyond the confines of their own niche and collaborate with the other niches. These barriers include differences in academic jargon and official and common parlance; lack of familiarity with the organizational structure of other niches, their hierarchy and formal and informal networks; lack of familiarity with the common codes of behaviour and how to address people; lack of time, capacity or inclination to work beyond routine in order to understand the other niche and perceptions of a status that does not match one's own niche status. This results in uncertainty at the individual level, whereas most people tend to seek safety and certainty.

To recapitulate: at the administrative and institutional levels, there is no common predefined objective that might bind the public health niches together. At the institutional and interpersonal levels, the general working methods differ widely and perceived barriers discourage collaborative efforts. Collaboration implies transformation of the relations between partners and change processes within the organization. 


\section{RESEARCH QUESTIONS AND DESIGN}

The general question underlying this dissertation is: which barriers prevent the collaborative development of public health practice, policy and research and how can the gaps between them be bridged? This question has been subdivided into the following, more specific, research questions:

1. What are the characteristics of the policy, practice and research niches and which steps within their work cycles are crucial for shared commitment, concerted action or joint decision-making?

2. Which plausible model of determinants can explain the lack of collaboration between policy, practice and research?

3. How can these determinants be influenced, based on theory, and what propositions can be made for practical strategies?

4. What can be learned about practical strategies from previous project-based collaborative structures?

5. Which practical strategies contribute to the development of collaboration?

A practical strategy is defined here as a specific technique for the application of theoretical methods in ways that it fits the population and the context (see chapter 3).

The niche phenomenon is not unique to the field of public health. For example, as far as public policy is concerned, a comparable gap between research, practice and policy is also apparent within sectors like public administration, traffic safety, social security, criminality/violence, women's emancipation, water management, infrastructure, education, employment, poverty, racial integration, etc. Since 1980, the policy-making process has become more interactive ${ }^{131}$, yet cooperation in collective endeavours is hampered by differences in the content, organization and culture of the three niches. This dissertation focuses on public health policy, but the insights thus gained might be transferable to other domains of professional practice, research and policy as well.

\section{MULTIPLE-CASE STUDY}

The most appropriate approach to answer the research questions seemed to be a multiple-case study. According to Yin ${ }^{132}$, case studies are a preferred strategy when questions of 'how' or 'why' are being posed, when the investigator has little control over events, and when the focus is on a contemporary phenomenon within a real-life context. A case study is a rather eclectic approach to reallife situations, where deliberate and active control over what is done to people is not feasible, even if it were ethically justifiable. This design is preferred when the object of study is characterized by great complexity ${ }^{132-134}$. In such cases, the 


\section{CHAPTER 1}

contextual circumstances are of great importance to the object of study. Case studies are in-depth investigations of specific units and are a useful way to explore phenomena that have not been rigorously researched yet, and especially to solve specific practical (real-life) problems. The role of theory development, prior to data collection, is one point of difference between case studies and related methods such as ethnography. The results of case studies are generalizable to theoretical propositions, not to populations, because the unit of analysis is not a sample from the population. The investigator's goal is to expand and generalize theories, rather than to determine frequencies. At the heart of the case study design is the idea that a case is studied in its own right, from a holistic point of view, to maintain the meaningful characteristics of real-life situations. Our research aimed to study the collaboration between policy, practice and research in the context of everyday institutionalized reality. The context of this collaboration is not clearly demarcated and often depends on the chosen subject and inherently also on the partners involved. It concerns a complex, contemporary phenomenon with many and varied key players. The outcome of a collaboration process is rather unpredictable and an investigator cannot directly, precisely and systematically manipulate the behaviour of the key players. Our goal was to make use of analytical generalization to propose a set of practical strategies for collaboration between policy, practice and research.

Compared to a one-case study, a multiple-case design has the advantage that the evidence is often regarded as more compelling. Overall, a multiple-case study approach is therefore regarded as more robust, provided that each case is still seen as holistic and embedded, and the findings are not pooled ${ }^{132}$. The cases selected for the present research project included project-based collaboration structures that were established during the 1991-2005 period. All cases were initiated by the GGD in the Southern Limburg region and were selected because they met the criteria of collaboration between policy (i.e. the local authorities in this region), practice (GGD) and research (Maastricht University and Maastricht University Hospital).

Six cases were analysed to study propositions with regard to elements stimulating collaboration. The unit of analysis is the 'case', to be described as a collaboration between policy, practice and research without restrictions on the strength of ties between the collaborative partners. Prior to data collection, theory and concepts were studied to develop an adequate model for the case studies. The cases and their outcomes were likely to be multifaceted and to be captured adequately only within a framework of different theories. The model summarized the theory-based practical strategies. These practical strategies were expected to change the determinants of collaboration. The theory-based practical strategies were evaluated by experts. Stakeholders for the expert meeting were identified by snowball sampling. They were selected from the fields of local policy, practice and research, and from national advisory bodies on health, 
such as the Netherlands Organisation for Health Research and Development (ZonMw), the National Institute of Public Health and the Environment (RIVM), and the Inspectorate for Health. The theory- and expert-based practical strategies provided clear guidance for the decision what data to collect. Data collection was based on documents and direct observations. Documents included annual reports, evaluation reports, official minutes of meetings and the articles enclosed in this dissertation. The strategy for analysing the data consisted of ordering the data in a matrix ${ }^{135}$ by tracing the proposed practical strategies in each case. These propositions helped to focus attention on certain data and ignore other data. This method also helped to organize the entire multiple-case study. Next, patterns were matched for the six cases, using collaboration as the dependent variable and practical strategies as the independent variables. If the results were as predicted, this method would allow solid conclusions to be drawn about the effect of the practical strategies on collaboration.

Case studies are one form of qualitative empirical research. Empirical research has to meet four quality requirements: trustworthiness, credibility, confirmability and data dependability. By contrast, parameters of design quality in quantitative research methods are internal validity, external validity, reliability and construct validity ${ }^{136}$. Internal validity deals with the soundness of the arguments (the data gathered) and the line along which reasoning has developed (the research design and analysis) ${ }^{137}$. A theoretical framework was developed to analyse the cases. External validity refers to opportunities to generalize the research findings. It can be properly ensured through virtual replication by using multiple cases to prove similarity in the results and theoretical replication by using a clear theoretical framework to test theories ${ }^{132}$. We analysed multiple cases to use the replication logic for analytical generalizations instead of statistical generalization. Virtual repeatability was used instead of actual repeatability, because exact replication is not possible in real-life situations ${ }^{137}$. To prevent threats to validity and reliability, the independent variables were defined as specifically as possible in concrete practical strategies.

In terms of evaluation research, this multiple-case study has contributed to the explanation of causal links in real-life interventions that are too complex for survey or experimental strategies. The dissertation describes effects of practical strategies for collaboration and finally provides an overview of practical strategies contributing to collaboration.

\section{OUTLINE OF THE THESIS}

Public health can be strengthened by collaboration between policy, practice and research, as is argued in chapter 1 . If local public health policy is to be supported by more solid evidence, this collaboration should arise at a local level. 


\section{CHAPTER 1}

Chapter 2 discusses the four steps in the work cycles of policy, practice and research, viz. describing the problem; deciding on a method; implementing the method and interpreting the outcome. Each work cycle starts with the identification of a problem, followed by a problem analysis and finding a method to solve it, which is then translated into an implementation design. Finally, the effects of the implementation are interpreted, which results in an evaluative conclusion about the contribution made by the method to solving the original problem. At first sight, these four steps are largely shared by the three domains, but a thorough analysis revealed many differences between the critical appraisal made by the practitioners, policy makers and researchers in each step of the work cycles. Important barriers that hamper collaboration are the disparities between the policy, practice and research niches, and the unequal paces of the work cycles. Bridging the gaps requires administrative authority, because the policy and practice domains are characterized by a rather hierarchic structure, and differences in some niche characteristics can only be overcome by influential and formal stakeholders. Next, organizational changes have to be made at the institutional level to facilitate individual behavioural change. Chapter 3 explores the determinants that explain the barriers to collaboration at the administrative, institutional and individual levels. Theory-based practical strategies are proposed which may contribute to a better understanding of the characteristics and work pace of each niche. The chapter ends with an overview of practical strategies which may promote collaboration at the administrative, institutional and individual levels. These theory-based strategies were submitted to experts from the fields of national and local policy, research and practice, who reflected on them in an expert meeting, as described in chapter 4 . The synthesis of the results of the expert meeting and the theory-based strategies was then used as a framework to evaluate the multiple cases. The extent to which these practical strategies were applied in the six public health cases that were studied is described in chapter 5. This multiple-case study evaluated the contributions and sensitivity of the various practical strategies. Concluding remarks are made on ways to bridge the gaps between the public health niches and their work paces. Chapter 6 provides a general discussion and recommendations. The figures 1.1 and 1.2 below outline the structure of the dissertation and the content of the six chapters. 
BACKGROUND AND PROBLEM DEFINITION

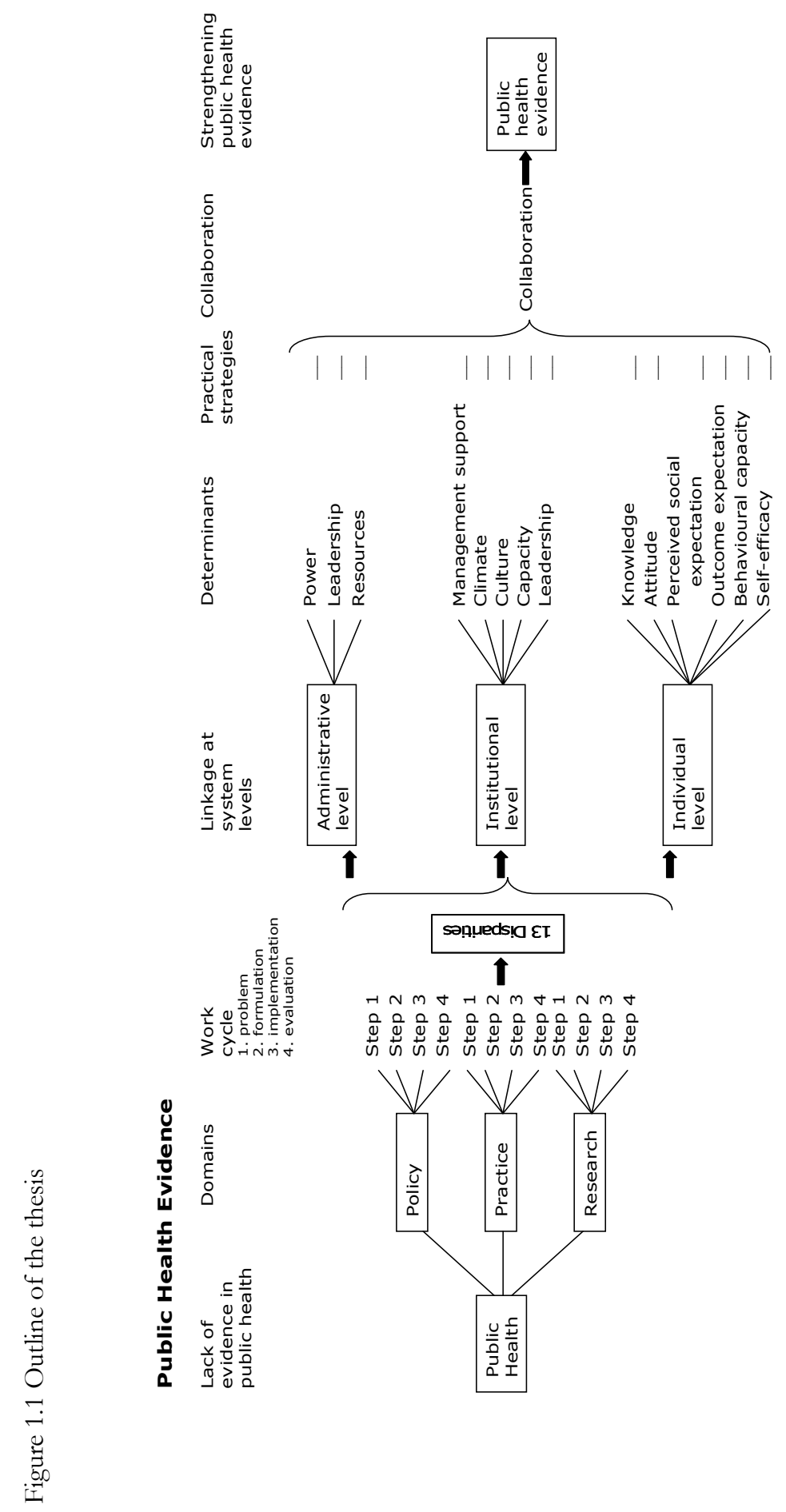




\section{CHAPTER 1}

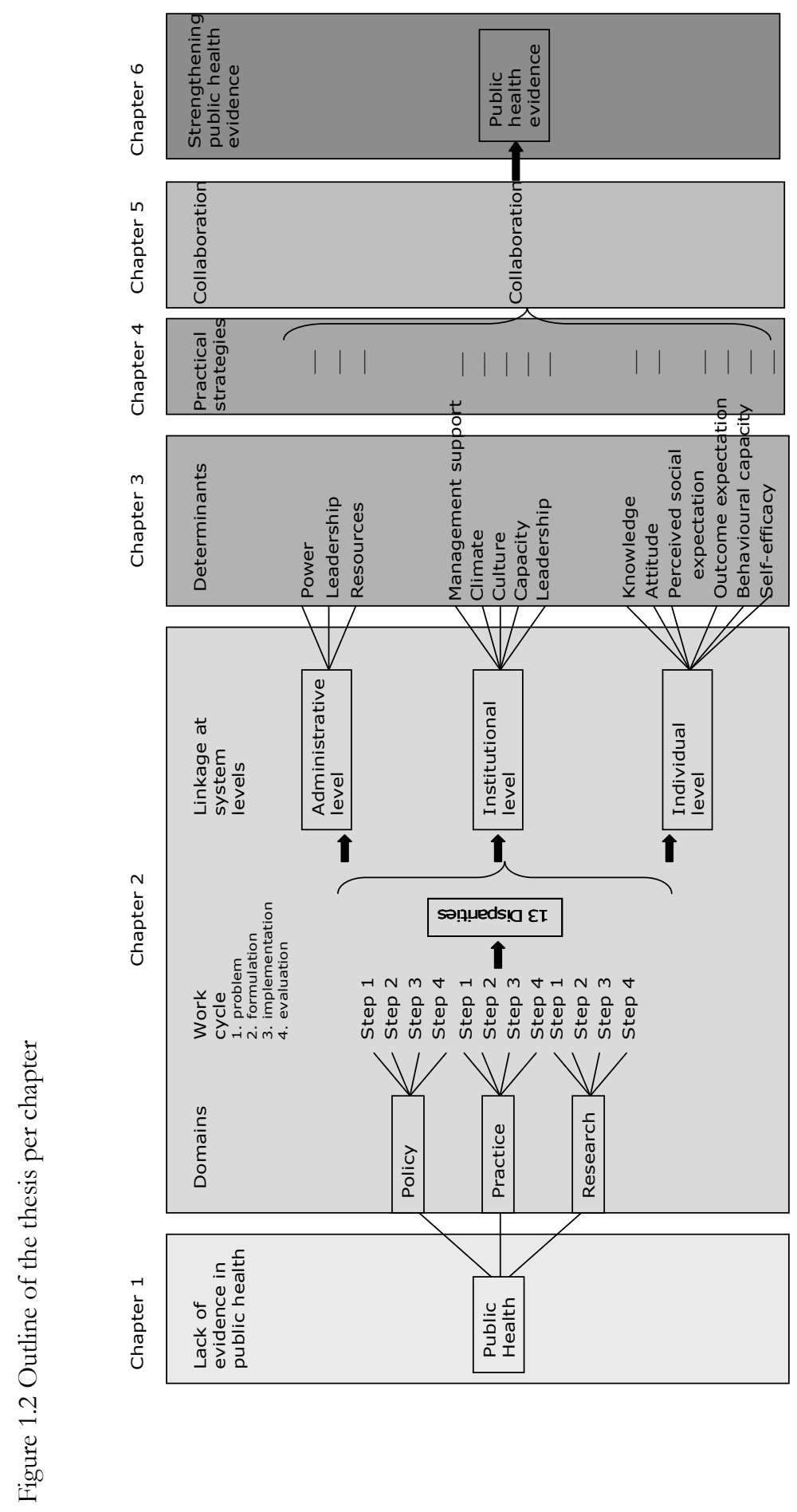




\section{Policy, practice and research}

\section{INTRODUCTION}

The professional activities within the policy, practice and research domains of public health can be characterized as work cycles. Such a work cycle shows a circular development pattern, with the actors and factors involved influencing each other and with a succession of clear-cut activities being recognizable. At first sight, the work cycles in these three domains seem to have much in common. In each domain, the first step is problem recognition, followed by an analysis of the problem and the formulation of an approach to solve it, which is step 2. Step 3 then involves the start of the implementation, and finally the effects are interpreted and evaluated in step 4 . Thus, each work cycle has the same successive steps: problem definition, approach formulation, implementation and interpretation. This chapter explores the successive steps of each work cycle, based on literature and expertise. It describes the frames of reference, professional standards, goals and characteristics of relevant stakeholders of policy, practice and research.

The work cycles are described according to the 'rational model', in which steps are sequentially organized. The current literature identifies three contrasting work cycle models of decision-making $87,138,139$ :

- a rational model, which is the classic interpretation that originates from rationalism and assumes that policies and decisions are made in a rational way, which means that policy makers, practitioners and researchers go through certain stages in a logical process to find the appropriate decision to achieve a predefined goal;

- an incremental model ${ }^{140}$, in which policy makers, practitioners and researchers negotiate with stakeholders about the goals and means, resulting in mutually agreed decisions consisting of small adjustments to the status quo;

- a mixed model ${ }^{141}$, in which 'high-order', fundamental decision-making processes are combined with incremental ones to overcome the shortcomings of both.

The rational model which we apply here was merely chosen as an instrument to structure the content of this chapter. As we discuss these work cycles it will become clear that the rational perspective is not a realistic reflection of reality, 


\section{CHAPTER 2}

and that the incremental perspective is in fact more prevalent. Work cycles proceed in a diffuse, open-ended, mistake-making interactive and iterative process $^{140,142}$.

This chapter aims to reveal the differences between the niches and the differences in pace, which are the main problems in the perceived interdependency between the domains. Finally, we assess those steps of the work cycle in which concerted action is crucial to achieve collaboration. Creating such concerted action requires administrative authority of the domains of policy and practice, in order to achieve a compromise that allows all three domains to simultaneously start with step 1. Concerted action in steps 2, 3 and 4 can be facilitated by organizational development at the institutional level, in order to motivate individuals to change their professional behaviour. Since the focus of this thesis is collaboration at the local level, the analysis in this chapter is presented from the perspective of local policy and local practice. The research work cycle is not specified in geographic or scale unit terms.

\section{POLICY CYCLE}

Policy is a long-term, continuously used, standing decision by which more specific proposals are judged on their acceptability in terms of means to be employed, ends to be pursued and the timeframe in which they have to fit. Public policy responds to social problems, in order to solve, reduce or prevent them. Basically, public policy is institutional, which implies that public problems can be solved by designing institutional actions, formal rules, procedures and routines $44,127,143$. The word institutional is used here to refer to the formal organizations that facilitate or restrict actors in their activities. In this dissertation, the term policy refers to public policy. Policy and politics each have a distinct role but are strongly interconnected, which is reflected in the description of the policy work cycle.

The sequential steps of the work cycle are the identification of a socially relevant problem (step 1), the formulation of an approach (step 2), the implementation of this approach (step 3) and the evaluation of the policy (step 4), see Figure 2.1, page 33.

\section{Step 1}

A problem is described as a discrepancy between negotiated and democratically defined, basic social principles and the current situation. The perception of problems therefore depends on a comparison between current normative standards and the real-life situation. Normative standards are influenced by the ruling political parties and public common sense. Problem definition is often interpreted as mainly a matter of strategic representation of the situation ${ }^{144}$. 
Figure 2.1 The regulatory policy cycle

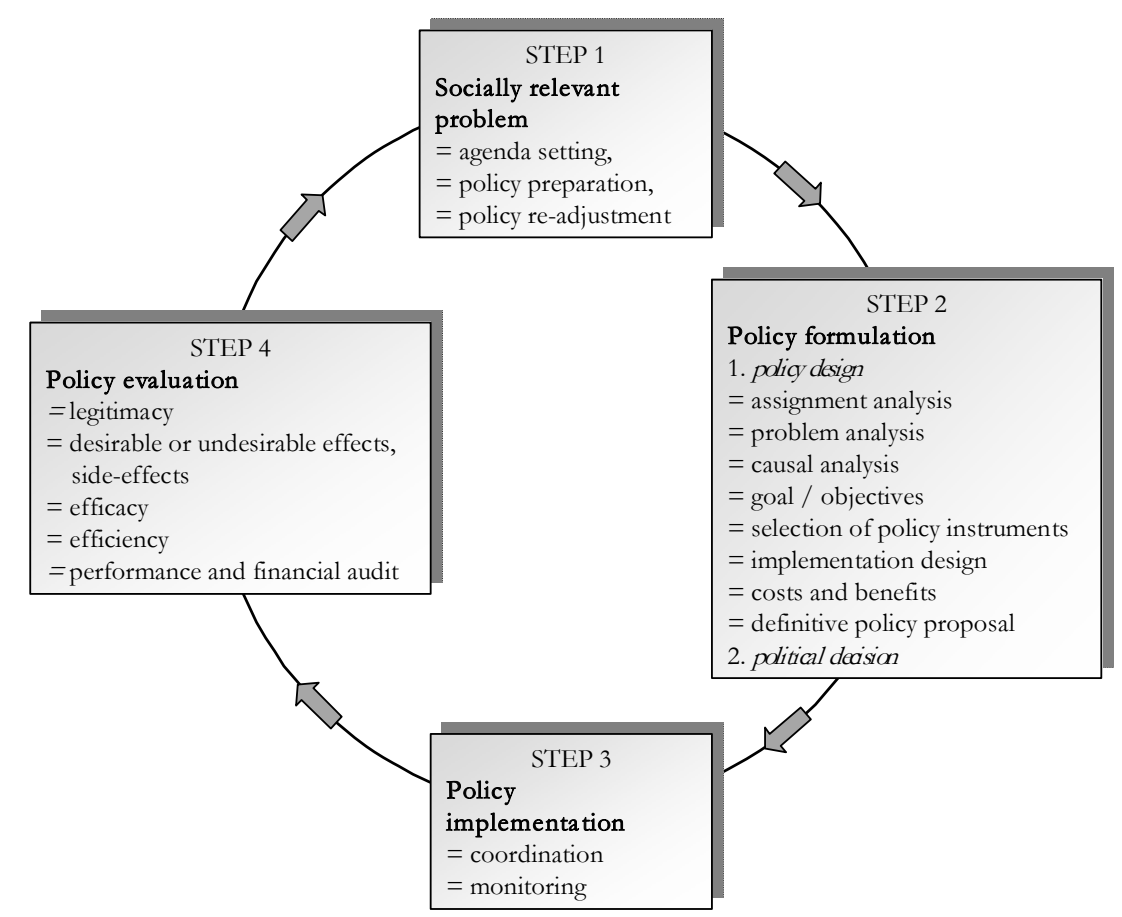

In the eyes of the public this may be regarded as window dressing or following the hype of the day. Agenda setting is a crucial aspect of step 1. How issues get onto the policy agenda or how they may be prevented from getting there because they go against the interests of those who are in power, is described in chapter 3 in the context of the theory of Kingdon ${ }^{145}$. Essential elements in Kingdon's theory are timing and social or policy entrepreneurship. Issues may be around a long time before they are propelled onto the policy agenda. Social and policy entrepreneurs may have an influential role in agenda-setting as they have formal and informal authority and they know how to perform in each domain. However, there seems to be no strong culture of social and policy entrepreneurship in the Netherlands with respect to public health ${ }^{112}$. Other important criteria for agenda-setting are whether the problem can be solved effectively, efficiently and efficaciously; whether causal determinants can be clearly identified; whether the involvement of the government is legitimated and the policy instruments are accepted by the prevailing political ideology and the majority of the population; and whether political salience, public visibility and personal immediacy are positively valued $30,48,146-149$. 


\section{CHAPTER 2}

Every four years, after the local elections, a new local government takes office and a new alderman may decide to put new problems on the political agenda. Lobbying for specific topics beforehand may influence the process of agenda-setting. The alderman responsible for the policy area and his or her political party are judged by the results achieved within a four-year timeframe. From the perspective of re-election, alderman's policy choices are influenced by the chances to be re-elected ${ }^{150}$.

\section{Step 2}

The formulation of policy (step 2) starts with an analysis of the policy domains that have to be involved. One has to decide whether a problem belongs to the education, public housing or public health department, or to all policy departments. Departments have their own legally defined authority status and informal power.

Designing a policy is inevitably subject to the influence of power, manifesting itself within the hierarchical structure of the policy process. The key players in local policy who decide on the design are the formal authorities, influenced by informal circuits. The formal power is in the hands of the mayor, aldermen and the municipal council. Policy proposals are prepared by civil servants, directed by the alderman responsible for the policy area involved. Strong leadership within the public administration will promote but may also hamper successful policy formulation. Strong leadership by an alderman will prevent civil servants from 'running the show', and from wheeling and dealing. Aldermen and councillors come and go, while civil servants stay in position when politicians are replaced. Civil servants may have huge power because of their expertise, knowledge and competence. They may even undermine political decisions, which is why they are sometimes called the fourth power ${ }^{129}$.

Public administrators take the complexity of the problem as a starting point. It is within the context of societal complexity that a policy becomes formulated, taking account of the complexity of the impact of solutions. The extent to which available knowledge is used to formulate policy to reduce complex social problems depends on the academic attitude of the civil servant and the knowledge management and knowledge infrastructure within the policy area, i.e. the specific department of the civil service that is involved ${ }^{139}$.

Councillors, mayor and aldermen are exposed to formal and informal external influences from the civil service, the media, advisory bodies, private investors, pressure groups or social movement groups and various private organizations ${ }^{44,131}$. Formal and informal contacts with persons who are accepted as respectable or authoritative by government policy makers belong to the political elite. This political elite has greater power, in the sense of legitimacy, information and coercion, than ordinary citizens, thanks to personal, affective ties ${ }^{36}$. Thus, policy formulation is closely affected by those who take part in it, both 
formally and informally. Real involvement of the target population and participation by intermediary organizations is often perceived as problematic ${ }^{131,151,152}$. Policy formulation can be characterized as a process of bargaining, lobbying, negotiation and the accommodation of different interests ${ }^{36,129,144,153,154}$. Step 2 also includes the selection of policy instruments, which can be divided into instruments directly affecting the target population, e.g., information, economic incentives (for instance subsidies) and legal directives, and institutional arrangements directly affecting the organizations made responsible for the implementation.

\section{Step 3}

Policy has to be implemented, depending on its resources and within a certain timeframe. The implementation phase is often insufficiently monitored. There is often no clear 'road map' showing what, where, when, how and by whom activities have to be implemented. Task allocation, responsibilities and competences are often left undefined. Constraints on implementation may be institutional impediments, lack of commitment amongst individuals or groups, unacceptability of policy by the target population because the policy solution is not their solution, excessive bureaucracy, insufficient freedom of implementation, insufficient resource allocation, inappropriate policy instruments, or a complex intersectoral network without hierarchy or administrative instruments to promote collaboration ${ }^{155}$. Despite the fact that local government claims to be in charge of local public health, the role of process manager often is unsatisfactorily implemented, especially when a range of partners from different sectors are involved ${ }^{156-158}$.

\section{Step 4}

The final step in the work cycle concerns the evaluation, ideally carried out using accepted methods and techniques of evaluation research. Policy evaluation is often considered unimportant, or at any rate, many policy programmes are not evaluated at all ${ }^{76}$. Specifically with regard to public health policy, there is no public accountability to estimate the impact of public policy on the health of the public ${ }^{159}$. Although evaluation of public health policy may face many problems there are methodological developments in establishing effect estimations and predictions ${ }^{160,161}$. In addition, policy decisions generally do not clearly specify the final goals, but merely state them in ambiguous terms of maximizing well-being, because the goal-seeking process serves consensus and cooperation, both in terms of party politics and with various external groups. Although such ambiguous goal formulation makes effect evaluations based on academic standards rather difficult ${ }^{144}$ it is possible to evaluate in terms of process criteria ${ }^{162,163}$. On the other hand, local government is obliged to audit its performance. Audits can be interpreted as an evaluation method. Audits are meant to 


\section{CHAPTER 2}

verify the degree of conformity with pre-defined requirements. Performance audits and financial audits are distinguished, both verifying the degree of conformity with defined performance indicators respectively budgets.

Evaluation research casts light on desirable and undesirable effects and side-effects, efficiency, efficacy, and - important for public policy - legitimacy, party politics, power strategies and public visibility ${ }^{164}$. These aspects play a rather important role in the accountability of civil servants to their aldermen ${ }^{165}$. Legitimacy during the formulation phase can be misjudged, can change over time or can be evaluated differently because of changes in political parties in power. Without public acceptance of government interference or the policy instruments selected, a policy can hardly be continued. Evaluation results add to the knowledge and expertise of the public administration. On the other hand, evaluation results are sometimes deliberately not published or communicated as this might be too risky to the political elite 129,144,164,166. Examples in the Netherlands include the Srebrenica debacle and the recent scandal about fraud in the Dutch construction industry.

\section{General}

The policy work cycle as a whole is a time-consuming procedure, taking up to four years (the time between elections). It is mainly carried out by civil servants, who are the bureaucrats of local government. This bureaucracy is the agency that administers the functions of the state in order to control the implementation, which is necessary for the political system. Civil servants are rewarded when they operate without failures, which may result in risk-avoiding behaviour. According to the eye of the beholder creativity is not a standard incentive within the policy work cycle ${ }^{167}$.

In recent decades, various models of policy construction have been developed, and applied policy science and the science of public administration have made considerable progress. The current view on policy-making assumes that the succession of steps in the work cycle is not sequential but rather disorderly. Each step can be followed by the next or can revert to the previous step, in what resembles an iterative process. This iterative model is more appropriate in an interactive, problem-solving policy-making process, and fits in better with the current complexity of social problems, than the classic interpretation of the sequential step model ${ }^{168}$. The step model should be understood as an idealized, normative or prescriptive model and the differentiation between the steps functions as a tool during the policy-making process. Lindblom ${ }^{140}$ has pointed out that this rational perspective is not realistic in social decision-making. Goals can not be predefined because of an abundance of values that are not agreed upon, an abundance of variables whose consequences cannot be controlled and the limited resources available to study all these values and variables. Therefore, Lindblom argues that what is feasible politically is only incrementally or margi- 
nally different from existing policies. This is not necessarily bad, because it concentrates the policy maker's analysis on familiar experience and thorough expertise. Stone even states that the advocates of rational policy decisionmaking "come close to promising that politics will become unnecessary"144 $\mathrm{p}$. ${ }^{241}$. She points out that policy makers can not always define the problem in detail, they do not have the time and information to allow them to make comprehensive predictions about the effects and efficiency of policy instruments, they are not value-free themselves, they have to work within administrative and bureaucratic constraints, they have to deal with political power conflicts, and past policy often determines present policy. Therefore, policy-making is very rarely revolutionary.

\section{PRACTICE CYCLE}

Practice literally means: the application of theory, or in other words, the accomplishment of activities based on theory. Practice aims to serve the needs of others, directly or indirectly. Practitioners in public health primarily want to solve problems and meet the needs and demands of their clients, with whom they have a direct, personal contact.

The sequential steps of the work cycle are the identification of a relevant problem in practice (step 1), the formulation of a practical programme (step 2), its implementation (step 3) and its evaluation (step 4), see figure 2.2, page 38.

\section{Step 1}

A problem is defined as a discrepancy between the actual situation and the needs and demands perceived by individuals, groups, communities and local authorities. The perception of problems depends on current normative standards compared to the actual situation as expressed in epidemiological findings, political priorities and public demands ${ }^{148}$. To recognize a problem, practitioners must perceive a difference between what currently exists in their consultation room or community and a more desirable state which they believe is attainable, modifiable and tractable. Problems in practice are concrete and detailed and practitioners focus on ways to act immediately, rather than on ways to reason or generalize, as researchers do ${ }^{169,170}$.

Every year, GGDs and local authorities make agreements on products to be delivered by the GGDs, in the so-called 'Diversified Products Provision Model' (in Dutch: Produkt-Keuze-Model). This process means that budgets and capacity are fixed. Product choices are determined by statutory regulations (Public Health Act, Infectious Diseases Act, Resolution Youth Care Act, Act on Disaster Control and Crisis Management), nationally assessed quality standards, and local public health policy ${ }^{60}$. The effect of the Diversified Products 


\section{CHAPTER 2}

Figure 2.2 The regulatory practice cycle

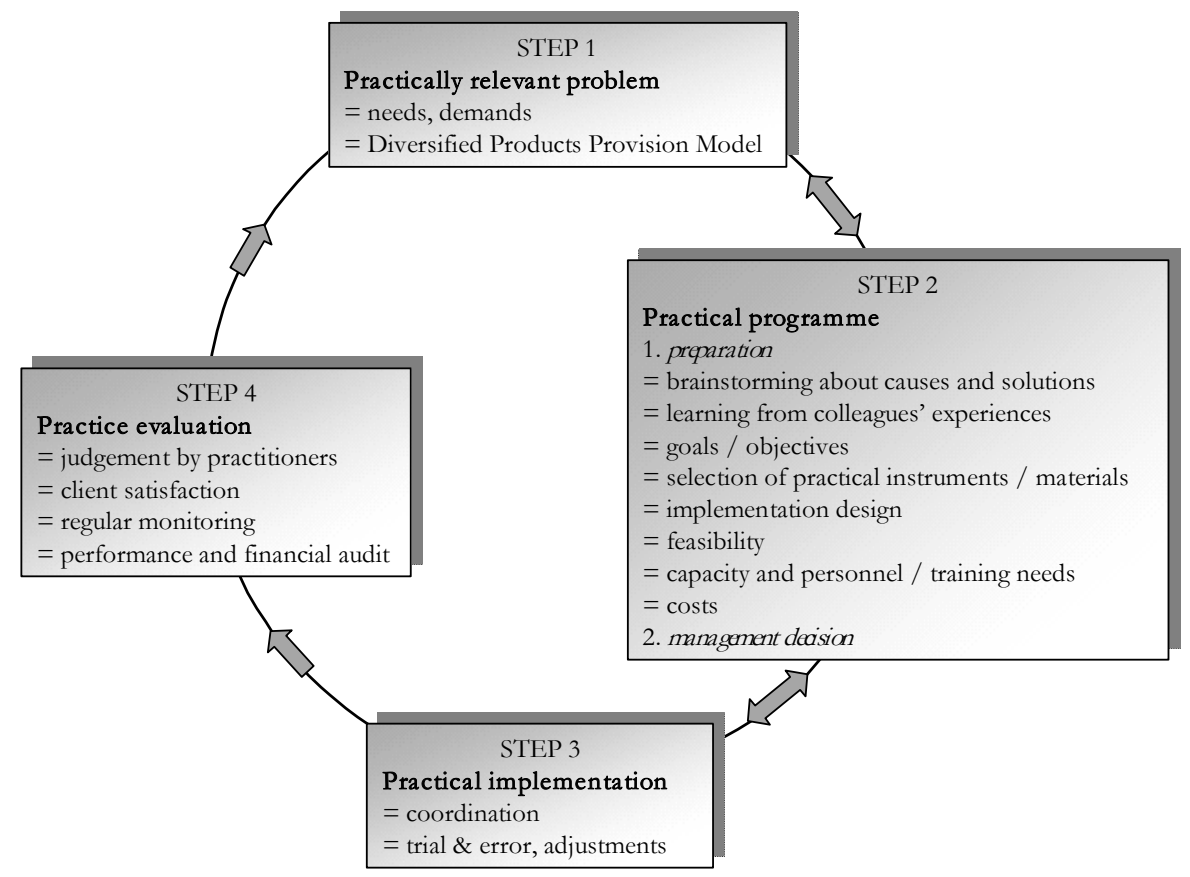

Provision Model is that GGD management can only make investments on the development of new programmes to solve identified problems when this is permitted by budget and capacity restrictions. Otherwise, negotiations with local authorities have to be initiated to increase budgets. After permission has been granted by the GGD management or the local authorities, step 2 can start. This implies a highly influential role of the local authorities.

\section{Step 2}

Practitioners almost always experience time constraints due to organizational and personal factors. The former relate to the practice domain as a going concern with an organizational duty to deliver products, fixed in scope and frequency, as determined each year in the Diversified Products Provision Model. Innovation is not a product in itself, unless the local authorities have decided to make innovation a priority in its own right. Personal factors relate to personal job satisfaction and professional attitude. Practitioners are intrinsically motivated to 'do' and to give meaning and status to their lives by directly solving or preventing problems ${ }^{169,170}$. The professional attitude involves the diagnostic process of predominantly non-acutely life-threatening diseases, although missing a diagnosis might have serious health consequences in the long run. The 
identification of individual problems by practitioners and the referrals they make are based on the method of differential diagnosis in which the optional causal probabilities are arranged in a decision tree and finally confirmed, rejected or falsified ${ }^{171-173}$. This procedure can be compared with the empirical cycle regarding the confirmation, rejection and falsification of a hypothesis. In case of non-specific complaints or ambiguous symptoms, physicians tend to retain their early hypotheses rather than reject them, even when subsequent facts support rejection ${ }^{174}$. Guidelines, based on medical technology assessment (MTA) or health technology assessment (HTA), are the best vehicles to introduce new insights into daily practice, so as to optimize patient care and problem diagnosis. Practice guidelines may be followed faithfully or occasionally ${ }^{175}$. The diagnosis of a problem at the community level requires an exhaustive behavioural, environmental and educational analysis ${ }^{176,177}$. Subsequently, the selection of practical strategies requires profound theoretical insights, which take a great deal of time to develop ${ }^{178}$. Practitioners, however, tend to regard theory as practically irrelevant and too time-consuming ${ }^{179}$ and their social commitment means that they want immediate solutions. Adopting ready-to-use practical strategies from colleagues is a fairly quick and easily accessible procedure ${ }^{180}$, which is often applied without a systematic validation of the context, as is recommended ${ }^{181}$. What is needed is a search for better and innovative ways of looking at problems and the development of strategies for change that are more effective to reduce current public health problems ${ }^{182}$. As innovative practice preparations require much time this tends to be discouraged by management. Furthermore, practitioners insufficiently anticipate the policy developments required to integrate programme activities in local policy ${ }^{96,152}$. Practitioners sometimes naively think that policy makers will automatically adopt their ideas because health is concerned ${ }^{179}$. Consultations about programme integration in local policy require long term discussions with civil servants.

Practitioners devote much attention to organizational constraints and the practical benefits of programme implementation. Their attitude makes them creative in terms of solving feasibility problems, unless resources or training facilities are totally inadequate. Agreement on the design is required by management. In the case of financial support or future budgetary risks local authorities have to decide, which results in decisions being delayed by some months, whereas the practitioners are in a hurry.

\section{Step 3}

If practitioners have designed the programme in close cooperation with all colleagues who have to use it, implementation is usually not a problem. Effective delivery may be impeded when the required capacity in terms of coaching and training is incorrectly estimated, the target population is insufficiently reached, no evidence-based interventions are used, or commitment and direc- 


\section{CHAPTER 2}

tives in a complex intersectoral implementation network are lacking. Early adopters (in Rogers' terminology) ${ }^{183}$ are in the frontline, driven by their enthusiasm and risk-taking behaviour. They serve as models, but function beyond routine practice, and their innovative strengths are liable to suffer from undermining activities by those who hold on to routine and conservatism, the socalled late adopters and laggards. Because a design almost never fits in perfectly with the working methods and contextual environment in which it is to become embedded, it has to be restructured and redefined. Incompletely worked out designs are improved by trial and error, sometimes resulting in 'muddling through'. Depending on the scope of the innovation and the available resources, practitioners generally want to turn a programme into a routine as quickly as possible.

\section{Step 4}

The final step of evaluation is an often neglected aspect in practical work, as it requires a theoretical rather than practical attitude. Evaluation is an attempt to assess the value of some service or approach, focusing on efficiency, efficacy, effectiveness, unanticipated outcomes, unanticipated processes, client satisfaction or job satisfaction. Evaluation can increase the quality and effectiveness of any initiative in practice by contributing to the processes of its planning, development and implementation ${ }^{184}$. However, evaluation comes at the bottom of the practitioners' list of priorities and budget items. At best, they might assess client satisfaction. Practitioners' work is not remunerated on the basis of health outcomes at the individual or population level, but on the basis of whether the Diversified Products Provision Model agreements are met. The WCPV regulations state that public health is to be monitored by the GGDs using health examinations and surveys amongst young people (0-19 yrs) and adults (18-75 yrs). However, these monitoring data are as yet not used for evaluation purposes.

GGDs are obliged to produce an annual report on their performance and financial auditing, under the so-called 'From policy planning to policy accountability' (Van Beleidsplanning Tot Beleidsverantwoording, VBTB) system. This planning and control cycle, however, functions as a productivity report rather than an evaluation of public health. It contains input data expressed in budgets, throughput data expressed in staff, and output data expressed in products. Outcome data, which can be documented in terms of life expectancy, prevalence of diseases or public health problems, are available from the two regular monitoring services but are not linked to the VBTB system. The effect on public health over time cannot be specified, quite apart from the general constraints and difficulties of measuring the effects of preventive public health services ${ }^{53,185-187}$. Any qualitative evaluative insight of public health practice in terms of a local trend may be attained by comparing the regular monitoring data with the 
VBTB over time, considering local circumstances and national monitoring data. Furthermore, academic research on programme effects is needed as the academic skills on the part of practitioners are insufficient.

\section{General}

The work cycle presented here is based on current practice within the Dutch context. Steps are passed through with a sense of urgency and the lifespan of the cycle is preferably as short as possible. The model proposed above describes an idealized situation. Not all practitioners will perceive the sequential steps. For some, work processes might even be linear as they follow instructions, while others work in a more disorderly way.

Strikingly, no applied scientific literature on the practice of public health in the Netherlands could be retrieved, apart from literature on quality improvement ${ }^{188}$ and a recently published book on the organization of effective health promotion ${ }^{189}$. The latter describes a common frame of reference on health promotion that can be used by practitioners to bridge the gap between them and policy makers and researchers. The book reviews the various problems practitioners, policy makers and researchers might face and offers alternatives to achieve unity in terms of language and modes of thought.

Evidence-based public health practice still is in its infancy. Of the 3000 projects which are currently being carried out in the Netherlands, only a small minority are sufficiently evidence-based ${ }^{190,191}$. The practice cycle as described here, intends to go beyond routine implementation. Inventive innovations require practice beyond routine ${ }^{192}$. In practice, some innovations create a high degree of uncertainty, which may provoke resistance. As a result, very few practice improvements are revolutionary.

\section{RESEARCH CYCLE}

Scientific research is defined as the systematic, controlled, empirical and critical investigation of hypothetical propositions about presumed relations among natural phenomena. Scientific research aims to produce explanations and predictions - and in case of applied science also solutions - relating to people's problems, and to add facts and theories to the body of knowledge. There are different ways to gain knowledge. One's own experiences represent a familiar and functional source of knowledge, closely related to the method of trial and error. Logical reasoning by induction (developing generalizations from specific observations) or deduction (developing specific predictions from general principles) is the next level of knowledge development. The scientific approach is the most systematized method of acquiring knowledge ${ }^{133,193}$. 


\section{CHAPTER 2}

In an idealized scientific study, the researcher moves in an orderly fashion from defining a scientifically relevant problem (step 1), through formulating the research question, designing the study (step 2) and collecting information (step 3 ), to evaluating and solving the problem (step 4), see figure 2.3, page 42. This orderly pattern is called the empirical cycle. The empirical cycle refers to the process in which evidence, rooted in objective reality (assuming that there is an objective reality that exists independent of human discovery or observation) and gathered through the human senses, is used as the basis for generating knowledge.

Figure 2.3 The empirical research cycle

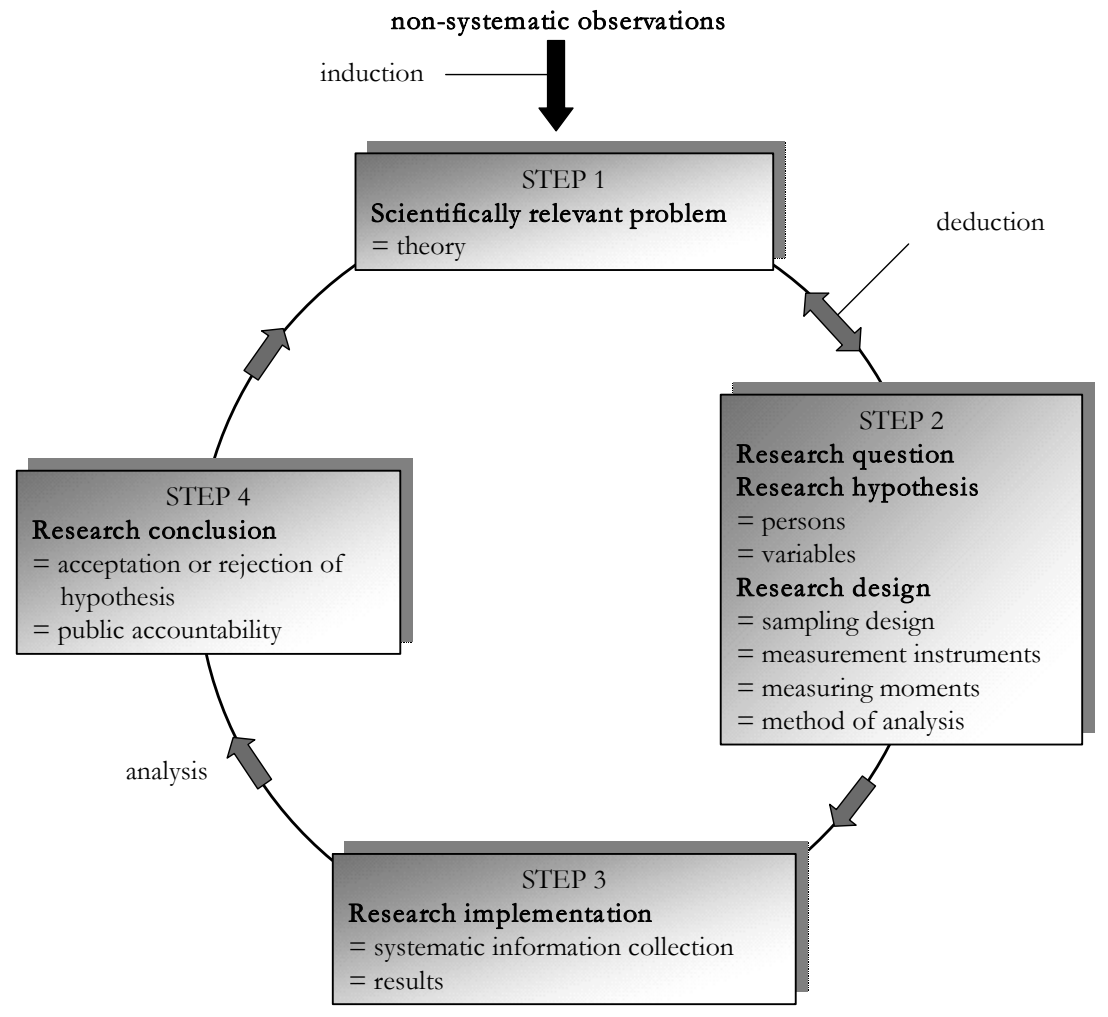

\section{Step 1}

In the research domain, a problem is described as a discrepancy between theory and reality, between different theories, between theory and practice, or between practice and desired practice ${ }^{193-195}$. A problem is perceived as scientifically relevant when, by systematic empirical observations, information can be accumulated or theories can be formulated to extend the existing knowledge base. The 
difference between basic and applied science is merely gradual, since many findings from basic research endeavours are ultimately applied to practical problems.

Step 1 is the generalization of non-systematic observations or perceived practical problems into a scientifically interesting problem that is based on theory. A theory is a set of interrelated concepts, definitions and propositions that presents a systematic view of events or situations by specifying relations among variables in order to explain and predict the events or situations ${ }^{133,196}$ p. 150 . Theories are by nature abstract, general, and testable, and originate from empirical data or observations that are generalized ${ }^{195}$. Concepts are the major components of a theory and are generally referred to as variables. The problem statement describes in detail what is the problem, why it is a problem, for whom it is a problem, and what causes are plausible and modifiable ${ }^{197}$. Problem statements originate from passionate researchers who integrate observations in a more abstract, generally valid picture of reality by creativeness and imagination. They prefer to do this without any hierarchical interference, which is perceived as an infringement of scientific autonomy. The most they will allow others to do is assess the research outlines, which may impose some restrictions on the research topics to be addressed.

\section{Step 2}

A hypothesis, formulated by deductive reasoning, is a tentative prediction or explanation of the relationship between two or more variables. The hypothesis serves as a link between the theory and the real-life situation. Descriptive, exploratory and phenomenological studies may not require a hypothesis beforehand, as their aim may be to develop hypotheses. The population to be studied and the variables involved are delimited. The research question(s) and the research design are thoroughly elaborated, which is generally a time-consuming process. Researchers tend to reduce complex problems to a range of more detailed problems that can be studied separately. Researchers need to be experts in developing the research design, sampling design, instruments to measure variables, timing and methods of analysis. There are many textbooks on principles and methods of different types of research $133,136,198-201$.

In public health research, humans are often the source of information, and great care must be exercised in ensuring that the rights of these humans are protected ${ }^{202}$. The procedure for obtaining informed consent needs to be addressed and implies extra time, pending the decision of a medical ethics committee.

\section{Step 3}

Based on the research design, the actual implementation of the research, corresponding to the empirical phase, can start. In many research studies, the em- 


\section{CHAPTER 2}

pirical phase is the most time-consuming part of the investigations, because of the large amounts of time that need to be invested for the data collection. Strikingly, many handbooks on research methodology omit general information about criteria for the feasibility of research implementation in a real-life setting. Researchers do not always sufficiently anticipate problems relating to matters like practical data collection or registration systems, controlled application and fidelity to the intervention in practice ${ }^{14,117}$, logistic requirements, identification of partners who need to be involved or committed, qualified staff, guarantees of soundness of the research design during implementation, recruitment procedures, resource availability, etc.

So far, most empirical implementation studies have been restricted to a particular context and content, which implies that hypothesis testing requires other empirical data from other universes to allow generalization and theorybuilding 203 . As the content, context and goals of the interventions vary considerably, there is an increasing demand for more implementation and diffusion research ${ }^{114,121,204-208}$. The scarcity of implementation and diffusion research ${ }^{209,210}$ results in insufficient evidence to allow innovations tried out in research to be introduced in practice or policy ${ }^{211}$.

\section{Step 4}

Testing hypotheses is essential in empirical investigations. Although such hypotheses can be accepted or rejected, it is inappropriate to speak in terms of definite proof, because this is incongruent with the limitations of the scientific approach. Scientists constantly seek objective, replicable evidence as a basis for understanding phenomena. The more frequently the same results are found, the greater the confidence. According to the eye of the beholder scientists are basically doubters, and this attitude results in conclusions with many nuances and poor applicability in policy and practice ${ }^{27}$.

Scientific researchers have a duty in terms of public accountability, and should communicate their findings to an audience. Four types of audiences are distinguished: the scientific forum, the institutions funding the research project, the practice forum and the public in general. Accountability to the scientific forum has a twofold function, i.e. to assess whether the results and the design can stand the test of scientific criticism, and to serve the body of knowledge ${ }^{201}$. The productivity of researchers is often assessed by the number of scientific publications in influential international journals with a high citation score. Due to this mechanism, publications in Dutch professional journals, specifically meant for the practice forum, are undervalued, impeding the dissemination of findings to practice. Reports and accounts intended for institutions funding the research project are usually not widely distributed. To address the public in general and politicians in particular, news media publications can serve political agenda-setting. Scientists are expected to retain a position of objectivity, keep- 
ing at a distance from policy or practice and avoiding public controversy ${ }^{212}$. Many researchers prefer to disseminate their results only within the scientific community. This conventional interpretation of the role of research is effectively keeping practice, policy and research apart ${ }^{22,30}$. To a growing number of scientists, however, their work is not restricted to the research setting and to the generation of knowledge. These scientists integrate the interpretation and application of knowledge in legislative, contextual and regulatory environments, while still emphasizing their objectivity and neutrality.

\section{General}

The steps described above represent an idealized conception of what researchers do. In actual reality, research rarely follows a neatly prescribed pattern of sequential steps. For instance, developments in step 2 might have consequences for the previously completed step 1 . Hence, just as in the policy and practice work cycles, the succession of the steps is not always strictly sequential, especially in descriptive and exploratory research. However, in explanatory research a rigorous implementation of step 2 before step 3 is of crucial importance and is a fairly standard procedure if a research project is to be eligible for funding. Due to the very time-consuming nature of this work cycle, careful prior planning and organization are essential, despite time constraints and deadlines. Research-based evidence has reached a fairly mature status as research methods have improved over time, for instance resulting in a growing body of knowledge about evidence-based medicine. However, evidence-based public health needs further progress. Progress in research is slow, step by step, and very few improvements in research are revolutionary.

\section{WHICH GAPS NEED TO BE BRIDGED?}

A review of these three work cycles and the description of current public health policy, practice and research of chapter one shows that there are some conspicuous disparities that strengthen the niche character. Improving the collaboration between the public health niches and their work cycles requires the following differences to be understood. Mutual understanding may subsequently reinforce mutual respect and collaboration.

1. Starting points are different as social, practical and scientific relevance do not automatically overlap. A synthesis of the different interpretations of 'relevance' is needed in order to attain the required interest from the practice, policy and scientific perspectives.

2. All local policy decisions, but not practical or research decisions, have economic consequences for municipalities, and major economic decisions are made by a small ruling elite, who decide to put a subject on the policy 


\section{CHAPTER 2}

agenda. Researchers and practitioners have no formal authority in local policy agenda setting, but they might play an influential informal role as social entrepreneurs.

3. Each niche is valued differently by the other niches in terms of social status. Policy makers, and even more so practitioners, assign high status to scientific research, due to its high intellectual requirements. Scientists and practitioners, on the other hand, perceive the policy-making process as highly bureaucratic, impenetrable, far removed from practice and rationality, and causing delay. Scientists generally perceive practice as socially relevant, but leave it to those with less academic capability, thereby implicitly lowering its social relevance and status. This perceived status difference widens the gap between the niches.

4. Practice and research have no formal decisionary power over policy formulation, whereas the political elite has. Occasionally, policy makers may invite researchers to intervene in the policy-making process by presenting objective, neutral, intellectual and valid information, free of political bias. Managers of private or public institutions sometimes belong to the political elite as social entrepreneurs, which may result in their exerting effective influence on policy formulation. Usually, however, the extent to which practitioners and researchers influence local policy formulation depends on their sources of power and is relatively limited.

5. The interconnections between the cycles are not clearly expressed. The process of decision-making in policy development is not a linear procedure from research to practice to policy. The same holds for the translation of research findings into practice. Instead, it involves a disorderly set of interconnections with back-and-forth steps that defies neat diagrams. It is therefore difficult to predict where the interconnections between the three work cycles will occur and to decide where these have to be made. However, a conceptualization of the possible interconnections and mutual expectations and a clear expression of the desired end state will contribute to interdependency and mutual obligations.

6. Evidence has different meanings in each cycle. The phrase 'evidencebased' refers to the conscious, explicit and judicious use of the best conclusive prevailing evidence that supports the decision-making process ${ }^{213}$. As the term 'evidence-based' is principally based on rationality, other interpretations of the term evidence have developed, reflecting the viewpoints of the parties concerned, as is obvious from the terms policy-based evidence and practice-based evidence180,214-221. The terms 'policy-based evidence' and 'practice-based evidence' add aspects that originate from the respective niche characteristics and consider the requirements and restrictions of each niche. This means that whereas rationality, empirical validity and logical precision are the decisive arguments for researchers, resulting 
in the concept of evidence-based research (which is a pleonasm), legitimacy, public acceptability, political salience, public visibility and public immediacy are important decisive arguments for policy makers to act or refrain from action, and these arguments shape the concept of policybased evidence. From the practitioners' perspective, meeting the needs of individuals and groups, as well as profitability and applicability, are the decisive arguments to act, expressed in the concept of practice-based evidence $7,16,24,25$. The different interpretations of evidence are often referred to as technical rationality against political rationality ${ }^{85}$.

7. Research and practice have so far had a focus on behavioural determinants, whereas policy instruments preferably should focus on the environmental determinants. Public health and health promotion research have put relatively great emphasis on personal determinants of health behaviour to influence lifestyle. So far, theories and research findings related to the environmental determinants have been unsatisfactory when it comes to formulating effective public health policy ${ }^{36,37,41,42}$. If the legitimate role of policy is to be linked to research and practice, the environmental dimension of health should be more explicitly defined ${ }^{57}$. Environment in this sense includes physical, social and cultural aspects. Policy instruments can contribute to making the healthy choice the easy choice because policy can legitimately use the environmental condition as a facilitator for behavioural change. Yet, it is a challenge to address the environmental determinants of health first and not to focus only on behavioural factors. Besides, policy actions focusing on behavioural determinants are considered moralistic and they may be politically controversial because they interfere in people's private lives.

8. The role and value of theory and practice are different in each niche. Theory is the starting point or the final goal of research and is regarded as indispensable. Within policy-making, the use of theory depends on the educational background and academic experience of civil servants. Practitioners tend to find theory rather irrelevant to their work, while researchers, on the other hand, tend to find practice-based knowledge scientifically irrelevant. The perception of theory and practice as a dichotomy has a long tradition in intellectual thought and has contributed to the divergence of the niches. Thinking in terms of a continuum or a synthesis will promote knowledge about public health evidence.

9. Scientists are regarded as thinkers, practitioners as doers, and policy makers as bureaucrats. These stereotypic images of each other's niches hamper collaboration, as they can become subconsciously fixed in people's minds. While research findings are often regarded as tentative by scientists, practitioners expect to receive clear guidance on how to act. A cautious scientific attitude may thus clash with a firm attitude towards action. Practitio- 


\section{CHAPTER 2}

ners may feel inhibited, while researchers must fight for the timeconsuming accuracy they strive for. The administrative function of the authorities often results in a controlling, bureaucratic attitude, which may conflict with the creative thinking and actions of researchers and practitioners. The political dimension that has to be taken into account by policy makers may lead to an opportunistic attitude that may conflict with the real-life problems practitioners are confronted with ${ }^{179}$. Each niche has arguments to act the way it does, and collaboration should combine the best of each of their approaches to achieve added value.

10. Adjustments during implementation are strongly discouraged in most research designs, whereas they are acceptable in policy and practice. As policy-related knowledge advances during the implementation stage (step 3) it influences policy formulation (step 2) and readjustments are made, sometimes causing practitioners and researchers to heave sighs of despair because the change process never seems to stop. However, once a research design has been selected and interventions have been defined, readjustments to the intervention are not allowed, sometimes demanding huge sacrifices and inflexibility from the practice field, which may even be confronted with client dissatisfaction ${ }^{222}$.

11. Each cycle has its own dynamics and lifespan. Some research projects, policies or practical programmes have a short lifespan, while others last a long time. Cycles end when, for instance, new research findings appear that disprove existing views, when practical implementation becomes counterproductive or routine, when the legitimacy of policy becomes undermined $^{223}$, or when the results are delivered without new problem statements arising. The policy work cycle in the Netherlands takes four years, i.e. from one local election to the next. However, unforeseen arguments within the political arena sometimes cause cycles to start during the period in between elections, and their duration can then hardly be predicted. The research project cycle takes about four years because $\mathrm{PhD}$ projects usually take four years. The practice cycle tries to speed up the process because practitioners feel a sense of urgency. This combination of different paces and the desired interconnections between the cycles (see point 6) makes meshing extremely complicated.

12. After a project has been evaluated, there is no longer a shared responsibility across the niches. After research findings have been evaluated, researchers no longer have a legitimate role related to their process criteria for accountability, leaving practitioners and policy makers to apply the research findings. As research tends to reduce the complexity of real-life problems to detailed sub-questions that are studied separately, it is often difficult to offer an integrated problem solution. Action on any substantial scale often has to wait for further analysis to address the complexity of 
contextual and institutional determinants in order to corroborate the evidence in practice or policy. During the initial phase, project conditions prevail, resulting in additional budget, training facilities, staff and other support. Continuation and sustainability are hampered by the fact that the additional support ends with the completion of the project, and the shared responsibility is dissolved.

13. Policy makers, practitioners and researchers each have a duty of public accountability, but in different ways. The reports required by the 'From policy planning to policy accountability' (VBTB) system and peerreviewed, scientific journals are, in theory, accessible to the general public, but access is hampered by a range of barriers relating to organizational structure and content. Data from process and effect evaluation are nearly exclusively used within the individual niches that produce them. The rate of intersectoral knowledge circulation is generally considered to be too slow.

These thirteen disparities are summarized in table 2.1. Numbers in the text and table 1 correspond and the thirteen disparities are classified in each of the four steps of the work cycles.

Table 2.1 Differences between the work cycles in the three niches

\begin{tabular}{|c|c|c|c|}
\hline & POLICY & PRACTICE & RESEARCH \\
\hline \multicolumn{4}{|l|}{$\begin{array}{l}\text { STEP } 1 \\
\text { PROBLEM }\end{array}$} \\
\hline 1. relevance & $\begin{array}{l}\text { socially relevant prob- } \\
\text { lem, i.e., solving social } \\
\text { problems }\end{array}$ & $\begin{array}{l}\text { practically relevant } \\
\text { problem, i.e. corre- } \\
\text { sponding to the public's } \\
\text { or client's requests or } \\
\text { needs due to problems } \\
\text { that are modifiable and } \\
\text { tractable }\end{array}$ & $\begin{array}{l}\text { scientifically relevant } \\
\text { problem, i.e. explain- } \\
\text { ing problems and } \\
\text { adding to the body of } \\
\text { knowledge }\end{array}$ \\
\hline 2. agenda setting & $\begin{array}{l}\text { influence on agenda } \\
\text { setting }\end{array}$ & $\begin{array}{l}\text { limited influence on } \\
\text { agenda setting }\end{array}$ & $\begin{array}{l}\text { very limited influence } \\
\text { on agenda setting }\end{array}$ \\
\hline 3. social status & bureaucratic status & $\begin{array}{l}\text { intermediate social } \\
\text { status }\end{array}$ & high social status \\
\hline \multicolumn{4}{|l|}{$\begin{array}{l}\text { STEP } 2 \\
\text { FORMULATION }\end{array}$} \\
\hline $\begin{array}{l}\text { 4. political in- } \\
\text { fluence }\end{array}$ & $\begin{array}{l}\text { influence of political } \\
\text { elite on policy formula- } \\
\text { tion }\end{array}$ & $\begin{array}{l}\text { sometimes indirect } \\
\text { influence on policy } \\
\text { formulation }\end{array}$ & $\begin{array}{l}\text { usually no influence } \\
\text { on policy formulation }\end{array}$ \\
\hline
\end{tabular}




\begin{tabular}{|c|c|c|c|}
\hline & POLICY & PRACTICE & RESEARCH \\
\hline $\begin{array}{l}\text { 5. interconnec- } \\
\text { tions and ex- } \\
\text { pectations }\end{array}$ & $\begin{array}{l}\text { insufficient transparency } \\
\text { of final goals and inter- } \\
\text { connections with re- } \\
\text { search and practice } \\
\text { cycles }\end{array}$ & $\begin{array}{l}\text { insufficient transpar- } \\
\text { ency of final goals and } \\
\text { interconnections with } \\
\text { research and policy } \\
\text { cycles }\end{array}$ & $\begin{array}{l}\text { insufficient transpar- } \\
\text { ency of final goals } \\
\text { and interconnections } \\
\text { with policy and } \\
\text { practice cycles }\end{array}$ \\
\hline 6. evidence & $\begin{array}{l}\text { policy-based evidence: } \\
\text { legitimacy, acceptability, } \\
\text { visibility, immediacy, } \\
\text { political salience }\end{array}$ & $\begin{array}{l}\text { practice-based evidence: } \\
\text { profitability, applicabil- } \\
\text { ity, feasibility }\end{array}$ & $\begin{array}{l}\text { evidence-based re- } \\
\text { search: rationality, } \\
\text { empirical validity, } \\
\text { logical precision }\end{array}$ \\
\hline $\begin{array}{l}\text { 7. legitimate } \\
\text { actions }\end{array}$ & $\begin{array}{l}\text { environmental ap- } \\
\text { proach, social, physical, } \\
\text { economic }\end{array}$ & $\begin{array}{l}\text { focus on individual } \\
\text { behavioural approach }\end{array}$ & $\begin{array}{l}\text { insufficient focus on } \\
\text { environmental ap- } \\
\text { proach }\end{array}$ \\
\hline $\begin{array}{l}\text { 8. value of theory } \\
\text { and practice }\end{array}$ & $\begin{array}{l}\text { theories are partly rele- } \\
\text { vant; } \\
\text { practical implementation } \\
\text { is relevant }\end{array}$ & $\begin{array}{l}\text { theories are irrelevant; } \\
\text { practical implementa- } \\
\text { tion is relevant }\end{array}$ & $\begin{array}{l}\text { theories are relevant; } \\
\text { practical implementa- } \\
\text { tion is partly relevant }\end{array}$ \\
\hline 9. work attitude & $\begin{array}{l}\text { work attitude of admin- } \\
\text { istrative control and } \\
\text { opportunism; some } \\
\text { creativity involved }\end{array}$ & $\begin{array}{l}\text { firm, action-directed } \\
\text { work attitude; 'quick } \\
\text { and dirty'; creativity } \\
\text { involved }\end{array}$ & $\begin{array}{l}\text { cautious work atti- } \\
\text { tude; detailed and } \\
\text { time-consuming; } \\
\text { creativity involved }\end{array}$ \\
\hline \multicolumn{4}{|l|}{$\begin{array}{l}\text { STEP } 3 \\
\text { IMPLEMENTATION }\end{array}$} \\
\hline 10. adjustments & $\begin{array}{l}\text { interim policy adjust- } \\
\text { ments }\end{array}$ & $\begin{array}{l}\text { interim practical ad- } \\
\text { justments; trial and } \\
\text { error }\end{array}$ & $\begin{array}{l}\text { no interim adjust- } \\
\text { ments }\end{array}$ \\
\hline \multicolumn{4}{|l|}{$\begin{array}{l}\text { STEP } 4 \\
\text { EVALUATION }\end{array}$} \\
\hline 11. lifespan & $\begin{array}{l}\text { unpredictable lifespan of } \\
\text { work cycle, maximum } 4 \\
\text { years }\end{array}$ & $\begin{array}{l}\text { preferably short lifespan } \\
\text { of work cycle }\end{array}$ & $\begin{array}{l}\text { predictable lifespan, } \\
\text { depending on re- } \\
\text { search design }\end{array}$ \\
\hline $\begin{array}{l}\text { 12. responsibility } \\
\text { after project }\end{array}$ & $\begin{array}{l}\text { policy implications } \\
\text { undefined due to com- } \\
\text { plexity; continuation } \\
\text { insufficiently anticipated }\end{array}$ & $\begin{array}{l}\text { practical implementa- } \\
\text { tion and contextual } \\
\text { implications still unde- } \\
\text { fined }\end{array}$ & $\begin{array}{l}\text { research task relating } \\
\text { to detailed sub- } \\
\text { questions; no further } \\
\text { responsibility after- } \\
\text { wards }\end{array}$ \\
\hline $\begin{array}{l}\text { 13. public account- } \\
\text { ability }\end{array}$ & $\begin{array}{l}\text { increasing public ac- } \\
\text { countability within own } \\
\text { niche }\end{array}$ & $\begin{array}{l}\text { limited public account- } \\
\text { ability; mainly financial } \\
\text { within own niche }\end{array}$ & $\begin{array}{l}\text { public accountability } \\
\text { by publications in } \\
\text { highly authoritative } \\
\text { journals within own } \\
\text { niche }\end{array}$ \\
\hline
\end{tabular}




\section{Concerted decision-making is crucial}

The above overview of disparities between the niches has ignored the nuances which certainly prevail in reality. It has pointed out the differences in terms of brief, simplified characterizations and stereotypes, mainly to address the difficulties that may have to be overcome when starting a collaboration process. Various cross-links are conceivable to bridge the gaps between the three niches. Theories of linkage, networks, coalitions and interorganizational relations confirm the positive effect of communication or network ties especially when groups are heterogeneous, as they reduce uncertainty and facilitate empowerment by interpersonal communication. Face-to-face encounters consistently emerge as the most efficient way to transfer knowledge and achieve higher quality $5,11,27,101,139,178,179,183,224-228$.

Personal ties should be put in the proper perspective of the steps in the three work cycles and the disparities between these three cycles. Some gaps between the niches can only be bridged by those who have legitimate power ${ }^{229}$. The niches of policy and practice are relatively hierarchic and bureaucratic in nature. When establishing public health policy, municipal councillors from various political parties have the final say on policy decisions that have been prepared by the mayor and aldermen. This preparation process involves contributions from civil servants, commissioned by the mayor and aldermen. After a policy has been decided upon, the councillors have to attentively monitor the quality of the policy implementation, and they may call the mayor and the aldermen to account in this respect. Councillors are members of the municipal council, while mayor and aldermen constitute the public administration and together they form the local government. The process from policy preparation to eventual decision-making is a hierarchic process, with civil servants at the bottom, civil service managers in between and the mayor, alderman and councillors at the top. In its activities in public health practice, a GGD is controlled by a board consisting of representatives of local government, in which the managing director of the GGD only plays an advisory role. Therefore, the obvious stakeholders who can effectively bridge the gaps between the policy and practice niches are the representatives of the local administrative level. They are the gatekeepers who exert a major influence on the rejection or adoption of planned changes. The research niche can be characterized as non-hierarchic, preferring academic autonomy. An authoritative stakeholder from the research field should be involved in the initial collaborative stages, in order to achieve commitment for collaboration at the highest system level. This stakeholder should be willing to act as a social entrepreneur and to negotiate at the local policy level about the possible contributions that could be made by research to policy and practice and vice versa. It does not matter whether such a stakeholder comes from the university board of governors (parallel to the adminis- 


\section{CHAPTER 2}

trative level) or from the level of research institutes. The stakeholder might be a university professor or a managing director of the public health research institute. At this highest level of negotiations strategic decisions are made.

To facilitate collaboration, organizational development is required at the institutional level, which is defined here as the formal organizations that facilitate or restrict actors in their activities. The main actors are the various managers, who have the responsibility to support and create the necessary preconditions for researchers, practitioners and civil servants to collaborate. At the level of institutional management tactical decisions are made, i.e. where preconditions are met. At the individual level the operational decisions are made. The involvement of the various actors is illustrated in table 2.2. Ideally, these actors collaborate in the vertical and horizontal direction, and sometimes also across.

Table 2.2 Matrix indicating the actors in the collaboration process

\begin{tabular}{|l|l|l|l|}
\hline SYSTEM LEVEL & $\begin{array}{l}\text { ACTORS FROM } \\
\text { POLICY NICHE }\end{array}$ & $\begin{array}{l}\text { ACTORS FROM } \\
\text { PRACTICE NICHE }\end{array}$ & $\begin{array}{l}\text { ACTORS FROM } \\
\text { RESEARCH NICHE }\end{array}$ \\
\hline $\begin{array}{l}\text { Administrative level } \\
\text { for strategic deci- } \\
\text { sions }\end{array}$ & $\begin{array}{l}\text { councillors, mayor, } \\
\text { aldermen }\end{array}$ & $\begin{array}{l}\text { GGD board and } \\
\text { managing director as } \\
\text { advisor or social entre- } \\
\text { preneur }\end{array}$ & $\begin{array}{l}\text { University Board, or a } \\
\text { professor or managing } \\
\text { director of research } \\
\text { institute as social }\end{array}$ \\
\hline $\begin{array}{l}\text { Institutional level } \\
\text { for tactical deci- } \\
\text { sions }\end{array}$ & $\begin{array}{l}\text { managers of civil } \\
\text { service departments }\end{array}$ & $\begin{array}{l}\text { GGD managers and } \\
\text { managers of other } \\
\text { practice institutes }\end{array}$ & $\begin{array}{l}\text { professors or pro- } \\
\text { gramme leaders of } \\
\text { research institute }\end{array}$ \\
\hline $\begin{array}{l}\text { Individual level for } \\
\text { operational deci- } \\
\text { sions }\end{array}$ & civil servants & practitioners & researchers \\
\hline
\end{tabular}

Step 1, and to some extent also step 2, of the work cycles needs the involvement of the actors at the administrative level, since these actors must commit themselves to addressing a socially, practically and scientifically relevant problem (point 1 in table 2.1) despite the differences between the niches. They should jointly decide to put it on the local public health agenda (point 2 in table 2.1), act as role models that will help reduce differences in perceived status (point 3 in table 2.1), recognize and confirm the added value of collaboration during policy formulation (point 4 in table 2.1) and articulate mutual responsibilities with regard to the work cycle sequence and interconnections (point 5 in table 2.1). This procedure needs a formal structure, with formalized agreements to meet mutual commitments as work cycles progress.

Other gaps between the niches are more easily overcome by interinstitutional and interpersonal connections in informal or semi-formal structures. These differences between niches concern the evidence question (point 6 in 
table 2.1), the nature of planned interventions (point 7 in table 2.1), the appreciation of theory and practice (point 8 in table 2.1), work attitudes (point 9 in table 2.1), implementation and adjustment procedures (point 10 in table 2.1), time span (point 11 in table 2.1) and evaluation and public accountability (points 12 and 13 in table 2.1). Personal ties provide gateways to the knowledge available in other niches and may result in affective ties, which in turn stimulate receptivity and commitment to the other niches. Professionals are thus given access to the internal structures of the other niches, their formal and informal networks, climate and culture, which can help them to cross niche barriers. Strong personal ties prevent domination of confrontational trends over co-operative ones $^{230,231}$. Formal and informal network ties and communication are balanced when collaboration exists at the administrative, institutional and individual levels.

As regards formal and informal ties, it is possible to identify the crucial moments for shared commitment, concerted action or joint decision-making. One important similarity between the work cycles concerns the recognition of a problem being the starting point for further action. Evidently, without a problem there is no reason to run the work cycle. The similarity of problem recognition as a starting point should be taken as an advantage. Step 1 of the work cycles requires joint decision-making as a formal basis for further collaboration. Subsequently, partners have to agree on step 2, i.e. research questions and designs, practical programmes and policy formulation, as well as their interconnections. Attuning the proposed working methods is one of the success factors in collaboration, as it creates compromises and commitment at the institutional and individual levels 232,233 . A solid implementation plan includes task assignment, training facilities and management arrangements, which makes step 3 relatively easy to carry out. Monitoring and regular feedback between policy, practice and research will suffice to solve everyday problems that are sure to arise. The final step, step 4, involves presenting the results. The challenge is to reach consensus about the evidence, to weigh alternatives, and to attempt to shape a new agenda transcending one's own niche responsibility. A new problem may be put on the agenda at the administrative level, leading back to step 1, in which the synthesis of practical, policy and scientific relevance has to be achieved once again. The links between step 4 and step 1 should be close, to anticipate continuation and to ensure the incremental evolution of public health by a series of concerted actions. The work cycle repetitions are associated with the process of revising and readjusting public health policy to attain a higher level of evidence. In other words, it is crucial to jointly start with step 1, i.e. the local problem. Next, it is in steps 1, 2 and 4 of the work cycles that it is crucial to make joint decisions. 


\section{CHAPTER 2}

\section{SUMMARY}

This chapter has analysed the work cycles of policy, practice and research, as they are carried out by the respective professionals. The existence of a special niche character was confirmed by an analysis showing thirteen disparities between the policy, practice and research work cycles. These disparities range from priorities in problem statements to legitimate power roles, appraisal of evidence, work attitudes, work pace, transparency of final goals and the attention devoted to evaluation and continuation. Our theory is that collaboration between the domains of policy, practice and research is hampered by these thirteen differences. Apparently, the niche character imposes restrictions, which make the individual behaviours of policy makers, practitioners and researchers incompatible, unless an interface is created that can overcome the differences. Building such an interface between policy, practice and research, something often recommended but so far never structurally realized, requires practical strategies to be developed to stimulate compatibility between researchers, policy makers and practitioners. As each work cycle starts with the recognition of a problem, the actors involved need to achieve a synthesis of social, practical and scientific relevance of local public health problems. The next chapter elaborates on the practical strategies to stimulate compatibility and offers a model, based on interpersonal interactions at the administrative, institutional and individual levels, which could help to unite the relevant niche perspectives. 


\section{Determinants, theories and practical strategies}

\section{INTRODUCTION}

The previous chapter analysed the disparities between the niches involved in public health, finally assessing the need to bridge the gaps between these niches. Based on this needs assessment, the present chapter addresses the question 'how to bridge'. It restricts itself to the field of public health, describing practical strategies that may foster the convergence of the heterogeneous niches. For this purpose it uses the Intervention Mapping framework ${ }^{178}$, which has a strong tradition in health promotion. The framework helps to systematically apply theories and empirical evidence to decide on intervention planning, implementation and evaluation. Intervention planning is defined in the present context as the successive implementation steps required to achieve collaboration, whereas the evaluation step is beyond the scope of this thesis. We have first developed a logic model. Logic models are graphic representations of the demonstrated or hypothesized causal relationships between concepts such as programme activities, programme output, and outcome or benefits ${ }^{178}$. Our model describes the relationships - not necessarily causal relations - between an organization's administrative structure, the institutional aspects, and operational preconditions to realize an interface between practice, policy and research. The model includes the determinants at each level, based on literature and expertise, and the change objective, which is collaboration. The model (see figure 3.1) formed the starting point for the intervention mapping procedure. Other models used to stimulate collaborative relationships are the DISC model (see chapter 4, the Schoolbeat case study ${ }^{234,235}$ which has been derived from the WIZ model236-240, and the Reference Framework by Saan and De Haes ${ }^{189}$. Although these models have much in common with the model presented here, they do not distinguish between the administrative, institutional and individual levels, and only serve as diagnostic tools. The distinction between higher and lower system levels is helpful in planning interventions to achieve collaboration between hierarchic working institutes, as each level makes its own contributions and the total system promotes reciprocity. Since we were interested in ways of bridging the gaps between niches and we searched for practical strategies to improve the diag- 


\section{CHAPTER 3}

nosed situation, we developed our own model. Based on this logic model, this chapter proposes theory-based practical strategies which are expected to change the determinants and subsequently address the current lack of collaboration. Effecting changes at multiple levels has a synergistic effect in creating and sustaining change.

This chapter first presents a short introduction to the logic model, followed by a discussion of each determinant at each system level, in order to allow methods to be chosen to change the determinants. Subsequently, to meet the objective of collaboration, it discusses how the methods were creatively translated into practical strategies. Intervention Mapping makes frequent use of the concepts of methods and strategies. In this context, a theoretical method is defined as a general technique or process for influencing changes in determinants, whereas a practical strategy is defined as a specific technique for the application of theoretical methods in a way that fits the target population and the context. Methods and strategies form a continuum that extends from abstract theoretical methods to practical strategies ${ }^{178}$. The list of proposed practical strategies at each system level was then adjusted on the basis of the opinions of experts in the field of public health (see chapter 4) and was finally used as a checklist in our multiple-case study (see chapter 5).

Figure 3.1 Logic model explaining factors that contribute to collaboration

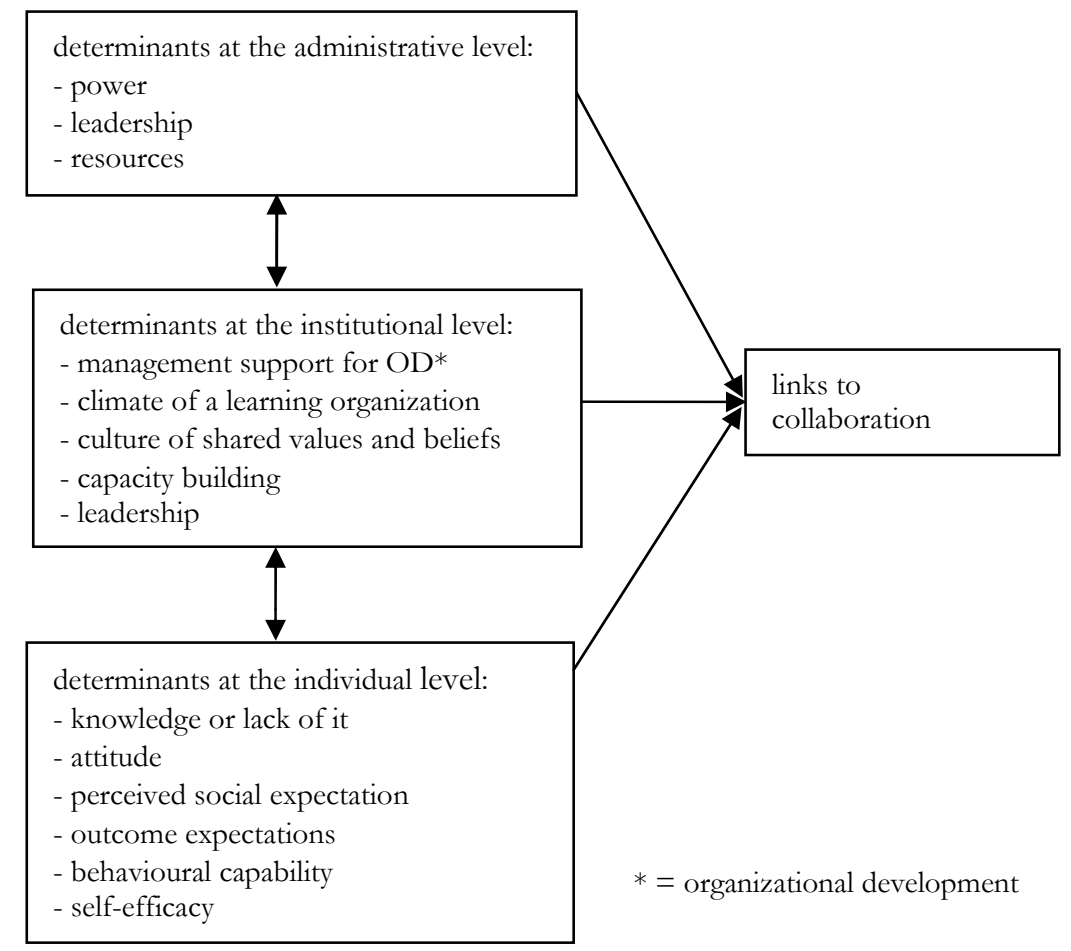




\section{THE LOGIC MODEL}

Collaboration is viewed as something achieved by individuals and the environment in which these individuals are embedded. In the case of the policy, practice and research niches, the environment includes the municipal administrative level, where decisions are made by the mayor, aldermen and councillors, the Board of the local GGD, and the University Board (the latter does not always operate as an actor because of the non-hierarchical structure of the university, and can be replaced by a social entrepreneur from the relevant research institute); the institutional level, where the managers are the main actors; and the individual level, including the professionals as the main actors. All the main actors operate as individuals within the environment. Based on the social ecology paradigm, there is a close relationship between individuals and their environmental conditions ${ }^{177,178}$. Each system level has its own internal hierarchy, structure and methods to influence individual behaviour. At the administrative level, power, leadership and resources are the main determinants of the success of attempts to influence or enforce collaboration. Practical strategies to change these determinants are advocacy, coalition building, lobbying, agenda setting and resource negotiations. These strategies are expected to contribute to solutions for the disparities between the niches. At the institutional level, the determinants include management support for organizational development to promote collaboration. Managers are the formal powerholders that come through the hierarchical organizational structure. Leadership is another important determinant within the process towards collaboration, and one which might be shared by niche members. Supported by the management, each niche leader has to (1) create the climate of a learning organization, acknowledging the reciprocity between the public health niches; (2) foster a culture of shared values and beliefs among practitioners, policy makers and researchers and (3) facilitate capacity building. These conditions result from performance objectives for managers at the institutional level and may remove barriers between the work cycles, thus promoting collaboration. At the group level, social influence occurs through interactions between the niche members, and such interactions change the determinants at the individual level. The main individual determinants relating to collaboration are knowledge, attitude, perceived social expectation, outcome expectation, behavioural capability and self-efficacy. Practical strategies to change these determinants will remove the barriers individuals perceive between the niches. The outcome of the interaction is then collaboration at the individual level.

The model illustrates the close relationship between the higher and lower system levels and indicates the reciprocity between professionals, institutes and boards of directors or governors. Although practical strategies may emerge at each system level, they incorporate the practical strategies of the lower levels. 


\section{CHAPTER 3}

Examples of practical strategies at the administrative level are advocacy and coalition building. These strategies are additional to the practical strategies of the institutional and individual system levels. Whereas strategic decisions at the administrative level create the conditions to facilitate or constrain institutional and individual change, individual behaviour can provide inputs to institutional and administrative changes. Individual behaviour may be shown by administrative officials, managers and practitioners, which implies that practical strategies at the individual level, such as knowledge or attitude, also apply to administrative officials and managers as individuals. Thus, each individual is influenced by, and can influence - directly or through an institute or board - the higher order system. For the sake of readability, the overview of practical strategies provided below only includes the new strategies that emerge at each higher order system level. It should be noted that these new strategies are the most important ones at the level concerned.

\section{ADMINISTRATIVE LEVEL}

Borrowing from the field of international relations, politics can be divided into high and low politics. High politics is defined as 'the maintenance of core values and long-term objectives of the state', whereas low politics is defined as 'not seen as involving fundamental questions relating to a state's national interest' 129,151 . The formalization of a linkage between policy, practice and research can be classified as 'low politics'. Policies that fall into the category of low politics are relatively open to special interest groups, making the linkage goal feasible.

At this level, three determinants are important related to the objective of collaboration: (1) power, (2) leadership and (3) resources. Theory shows that there are effective practical strategies to enforce a planned change towards collaboration.

\section{Power}

French and Raven identified six key power bases ${ }^{241}$ : reward power (the capability of controlling the distribution of rewards given or offered to the target), coercive power (the capacity to threaten and punish those who do not comply with requests or demands), legitimate power (authority that derives from the powerholder's legitimate right to require and demand obedience), referent power (influence based on the target's identification with, attraction to, or request to the powerholder), expert power (influence based on the target's belief that the powerholder possesses superior skills and abilities) and informational power (influence based on the potential use of informational resources, including rational argument, persuasion or tactical data). Greiner and Schein added 
the power of gaining the support of others ${ }^{242}$, as gaining the support of other key powerholders allows a critical mass for change to be achieved. Power is considered to be a relevant determinant in starting or avoiding collaboration between practice, policy and research.

The legitimate powerholders in public health often lack sufficient information and expertise on public health but may have reward, coercive or referent power. Researchers or practitioners at this level may also have referent power, which gives them the ability to put agreed policies into practice with consensus. Since the decentralization of Dutch local public health policy (1989), the policy makers and managing directors of the practice field often experience power conflicts. Directors from the practice domain have informational and expert power to advise on public health policy, while civil servants have the legitimate power to advise the local authorities and they have detailed knowledge of the procedures as well as key persons and relevant other policy domains. Civil servants may try to avoid collaboration between policy, practice and research, and they may seek to preserve the status quo in order to prevent that the informational and expert power of practitioners becomes further strengthened by the informational power of researchers. In other cases, the combined power of policy and practice is used to exclude the research domain, especially when political priorities require immediate action. The urgency is then used as an excuse to avoid a solid scientific analysis and evaluation. Practitioners and policy makers may also resist research that is critical of the things they have been doing ${ }^{27}$. Research and practice can also be too closely connected, using their combined power to set policy from behind the scenes, and suggesting that policy makers can understand neither research nor practice.

To change power relations the practical strategy of advocacy, or 'playing it straight', is the most widely used and successful strategy in organizational development ${ }^{243}$. Effective advocacy is based on legitimate, referent, informational and expert power. Since legitimate power can be lost, the other three are key assets. Advocacy may include methods of information, persuasion, negotiation or coercion. Advocacy may result in agenda setting, being the first step in the change process. Using advocacy, stakeholders must anticipate power changes to minimize the risk that one group will block changes.

To preserve the power balance, advocacy should acknowledge the historical roots of organizations and describe a common desired future, in which the synergism of the collaboration can result in power gains to each partner. In this situation, the core value of each organization (i.e., practical implementation of local public health policy by the local GGD, research and knowledge development by university staff and policy assessment by the local authorities) remains intact and each organization maintains autonomous programmes. The challenge is to create an envisioned future that originates from the synergism, to make programmes more evidence-based 244 . 


\section{CHAPTER 3}

\section{Leadership}

Leadership has been described as a specialized form of social interaction: a reciprocal, transactional and sometimes transformational process in which cooperating individuals are permitted to influence and motivate others to promote the attainment of group and individual goals ${ }^{245}$. This definition emphasizes the features of reciprocity, transactionality (i.e. the social exchange process), transformation (i.e. the change of members' beliefs, needs and values), cooperation and the goal-seeking process that motivates group members. Leadership is widely recognised as a critical success factor for the development of new collaborative networks ${ }^{246}$. Theories on leadership highlight the role of policy and social entrepreneurs, especially at the administrative level. In our situation, policy entrepreneurs are influential people who have sufficient background and credentials to understand the culture and methods of different local authority domains and the niches of research and practice ${ }^{119,145,225,247-250}$. Social entrepreneurs are stakeholders who emanate the notion of social responsibility; they value social justice and seek innovative solutions for socio-economic health inequalities. Social and policy entrepreneurs can have a significant impact on agenda setting. The absence of collaboration between policy, practice and research is thought to be mainly caused by the absence of social and policy entrepreneurs ${ }^{112}$. Influential persons both from research and practice may act as social and policy entrepreneurs, using referent, expert and informational power to stimulate commitment and reciprocity.

The local government, which has control over legislation and the funding of local public health policy, can be assumed to be the most influential agenda setter. Pressure from mass media and the public seems to be effective as far as low politics is concerned and can help to get issues on the political agenda. How issues reach the local government's policy agenda is a rather complex process, which is often highly unpredictable. According to Kingdon ${ }^{145}$, policy is made through three separate streams: the problem stream, held by problem owners; the policy stream, held by problem solvers; and the politics stream, held by formal decision-makers. The problem stream involves the recognition of problems, which is influenced by a number of factors, such as the magnitude and seriousness of an issue, a shocking event or a crisis. The politics stream is characterized by hidden participants, visible participants like the ruling political parties, each with their own political ideology, and the political mood of the citizens. Hidden participants are the specialists, like researchers who come up with interesting findings and who may 'leak' information to the media. The policy stream selects proposals and it only defines an existing condition as a problem if the problem solvers feel, or others persuade them to feel, that something needs to be changed and needs the attention of government officials. Problems and policies are only taken seriously by government when a 'window of opportunity' opens up in each of the three streams. The main point Kingdon 
makes is that the individual streams of problems, policy and politics have lives of their own. The independent streams flow through the social system. At certain moments, the streams will cross and if they all meet, a 'window of opportunity' opens. Politicians do not proceed from an identification of problems that have to be solved, but they await opportunities. Solutions to problems may be advocated for long periods before the opportunity arises for them to be accepted. A window of opportunity is only open for a short time, and policy or social entrepreneurs must adequately intervene at this most opportune moment. The theory of streams stresses the importance of policy or social entrepreneurs in linking partners coming from each stream. Powerholders and leaders from the problem stream, such as professors and GGD managers, can lobby and establish links to the policy and politics streams, allowing them to contribute to agenda setting. The contribution may be even greater when powerholders or leaders belong to the inner circle of local government. The alderman responsible for public health is assumed to have the greatest influence.

Stakeholders at the administrative level have to negotiate at the policy practice - research interfaces to build coalitions, attain consensus, articulate the mission and justify and consolidate the work of the institutions and individuals they represent. Coalitions are inter-organizational, cooperative and synergistic working alliances, which have been defined as organizations of individuals representing diverse organizations who agree to work together to achieve a common goal. Coalitions may be categorized by the type of membership (e.g., community members, professionals), patterns of formation (e.g., in response to an opportunity or a threat), types of function (e.g., resource sharing, technical assistance, planning and coordinating services) and types of structure that accommodate these functions (e.g., coalitions set up by organizations, networks, ad-hoc coalitions) ${ }^{233}$. To attain a collaboration between the three niches, the coalition members are professionals and policy makers who share public health goals, in an organization-set coalition that provides resources, services and mutual trus $\mathrm{t}^{230,231}$. Theories on coalition building describe the need to identify points of entry into each niche. The points of entry are: reducing the current public health decline by promoting evidence-based public health; pooling human and material resources; increasing the available capacity with a wider range of talent; minimizing duplications, maximizing power and achieving critical mass to achieve changes that cannot be brought about independently 251 .

\section{Resources}

Implementing a collaborative structure between policy, practice and research requires additional financial and human resources. If these extra resources are budgeted for and provided, the planned change is likely to occur. There might be an opportunity to apply for grants to finance additionally required shortterm investments during the transition stage. So far, project subsidies have 


\section{CHAPTER 3}

often allowed projects to get started, while leaving future finances undefined, which has often resulted in disappointing project termination. Therefore, longterm financial support must be covered by earmarked internal budgets. Decisions on budgets belong to the internal affairs of each organization. Agreement on the common goal and the planned change includes that all stakeholders have allocated budgets at their disposal, as financial problems might curb a stakeholder's enthusiasm. Stakeholders can use their formal power and make decisions in a top-down fashion, followed by internal negotiations on budgets with the organization's managers. Lack of lower management commitment can weaken the enforcement of the planned change.

\section{INSTITUTIONAL LEVEL}

The determinants affecting the initiation of a change process towards collaboration at the institutional level are: (1) management support for organizational development, (2) changes fitting in with the organization's climate, culture and capacity, and (3) leadership 211,252 .

\section{Management support for organizational development}

Management support for organizational development is necessary to facilitate the transition towards collaboration. An organization is a stable system of individuals who work together to achieve common goals through a hierarchy of ranks, division of tasks, and predetermined rules. Organizations are created to handle large-scale routine tasks through a pattern of regularized human relationships. The relatively high degree of structure in communication patterns and concerted human endeavours is in part due to this stability ${ }^{183,229}$. Lewin's change model states that there are forces that try to maintain the status quo and forces pushing for change because innovations (or improvements) are fundamental to the organization's survival. Implementing organizational change involves moving from the status quo to the desired future state and requires organizational development strategies (OD). OD has been characterized as 'a system-wide application of behavioural science knowledge to the planned development, improvement and reinforcement of strategies, structures and processes that lead to organizational effectiveness'. Lewin's model has been further worked out, resulting in a more comprehensive action research model in which different organizational and interorganizational development theories can be used in an eclectic approach to explain environmental influences on individuals and groups ${ }^{243}$. The action research model considers planned change as a cyclical process in which initial research (i.e. the diagnosis) about an organization - or in our case collaboration - provides information to guide subsequent action planning, intervention and evaluation. This model emphasizes the need for 
members of the organization to participate in the change process, plan changes and perceive ownership. Ownership is the opposite of resistance to change and refers to the willingness to take responsibility for the diagnostic data, the problem, the lack of capacity, its consequences and the need to reframe the reference ${ }^{110,243,252}$. Action planning includes three major aspects: activity planning (producing a road map that cites activities and interventions that must occur and that has been approved by top management), commitment planning (identification of key players whose commitment is needed and formulation of a strategy for gaining support) and change-management structures (a chief executive, a project manager, formal managers etc., whereby these key actors can change depending on the stage of the process). A communication design which clearly formulates communication channels is essential in keeping all partners involved. Process management and evaluation are important to monitor progress and to successfully adjust to goals, resources and internal supportive policies $^{156,253}$.

\section{Climate}

Theories on organizational development differentiate between organizational climate, culture and capacity 252 . Climate stresses the organization's 'personality', that is, how the organization is seen by outsiders. Collaboration between policy, practice and research requires an open-minded, actively learning and innovative organization that is willing to go beyond routine ${ }^{175,254,255}$. Structuring an open, learning climate requires formal statements and a policy of encouragement and reinforcement by rewarding initiatives and offering incentives, supported by PR policy (e.g., mass media, organizational media portrayals, behavioural journalism). Enabling factors such as knowledge management and knowledge infrastructure will strengthen the 'lifelong learning' climate ${ }^{139}$.

\section{Culture}

Culture has to do with shared beliefs and values among members. When researchers, practitioners and policy makers do not believe in the synergism of concerted actions, their collaboration will prove a dead end. The recognition of interdependency should be an acquired, shared value, as should mutual respect for the frames of reference of the other niches. Representatives of the research, practice and policy niches could be linked up, to experience each other's culture through short internships and to familiarize themselves with the organizational structure of the other niche, its hierarchy, social status, codes, norms and prejudices against other organizations. Cultures may move closer together, which can further facilitate the collaboration process ${ }^{142,256}$. Social support, increasing reciprocity (e.g., by material support or advice) and formal and informal consultation structures will contribute to a common culture of mutual understanding. 


\section{CHAPTER 3}

\section{Capacity}

Capacity building is essential to enhance the knowledge, skills, resources and commitment of key actors across research, policy and practice niches in order to reach a higher public health standard that is more responsive to community needs ${ }^{252,256-266}$. What is needed is the creation of a 'generic' capacity to tackle different problems, encouraging creative, divergent thinking and reflection amongst practitioners, researchers and policy makers, by providing a two-way flow of knowledge. The organizational diagnosis is the starting point for an inventory of specified capacity needs ${ }^{12}$. Feedback on these diagnostic data can ensure that individuals recognize the data, resulting in a sense of ownership. Organizational capacity is enhanced by being operationalized in job descriptions, job titles, recruitment of qualified staff, a professional development programme and human resource management ${ }^{19,119,256,267}$.

A kick-off conference may stimulate those involved to seek opportunities to incorporate policy, practice and research principles in management and staff members' own areas of expertise. It can help to increase understanding of the collaboration and may contribute to capacity building by reducing social barriers between the niche professionals through informal contacts.

Resources are central to organizational capacity building. This includes financial resources as well as infrastructural resources like time to study and access to tools such as libraries and publications, knowledge transfer through a clear knowledge infrastructure, availability of software packages and other services 256 .

\section{Leadership}

Just as at the administrative level, leadership is of eminent importance at the interorganizational level. At this level, leadership behaviour must be visible, as it functions as a role model. The style of leadership and management must foster lifelong learning and creative, divergent thinking, and encourage critical reflection, provided by exchanges between practitioners, policy makers and research$e^{142,268}$. Members of the three niches should learn from their mistakes instead of being punished. The theory of situational leadership states that leaders should be flexible. During the transformation to an interorganizational group of policy makers, researchers and practitioners, a leader should use a rather directive behavioural style, but over time, as the group develops, such a leader should shift to a more coaching, supporting and delegating style. A persuasive leadership style is preferred to an authoritative style $230,245-247,250,253$. Relevant behaviour by leaders to enhance innovative behaviour by co-workers involves offering intellectual stimulation, stimulating knowledge diffusion, providing vision, consulting, delegating, providing support for innovations, organizing feedback, recognizing, rewarding, providing resources, monitoring and assigning. 


\section{INDIVIDUAL LEVEL}

At the individual level, the outcome is mutually respectful co-operation between practitioners, policy makers and researchers, as a basis for intersectoral collaboration to improve the quality of the evidence on which public health should be based. There are many theories to explain individual and interpersonal behaviour. These theories have several constructs in common: knowledge and awareness, attitude, social influence and self-efficacy. Suggested additional determinants to improve the predictive validity for behaviour include moral norm, self-identity, habit, emotional factors, past behaviour and environmental factors ${ }^{269}$.

A typical characteristic of a niche is its close ties with the outside world. Knowledge, attitude, perceived social expectations and outcome expectations seem to be important personal determinants of both judging one's own frame of reference, for instance to improve evidence, and of entering another niche. The latter is also determined by behavioural capability and self-efficacy.

\section{Behavioural determinants}

Knowledge relates especially to the work cycle (i.e., the process) in a particular niche. Discussions surveying niche differences, like that presented in chapter 2 (see table 2.1), may help to alleviate an existing lack of knowledge. Group discussions offer opportunities not only to fill knowledge gaps, but also to learn about one another's operational processes and to reflect on one's own operational processes and professional standards. Written materials and training can contribute to an increase of knowledge.

Attitudes are positive or negative reactions to something, for instance collaboration, or to a specific behaviour. Two aspects should be distinguished: work attitudes in the different niches, which vary widely (see chapter 2 ), and the attitude towards the planned change, i.e., the process of collaboration. Practitioners, policy makers and researchers might not be convinced of the advantages of collaboration, an attitude which may be strengthened by unfamiliarity with the other organization's structure and its formal and informal networks, by personal interest, by their own past behaviour and by conservatism.

Perceived social expectations refer to the beliefs among policy makers about the way their colleagues expect them to act or behave and the way researchers and practitioners expect them to behave, as well as the motivation to comply. The same goes for researchers and practitioners. As a result of perceived status differences, different opinions about the role and value of theory, and different opinions about evidence, people may feel very uncomfortable and indecisive in a collaborative setting.

Outcome expectations refer to judgements about the likely consequence that a particular behaviour will produce, and evaluations of this outcome. When 


\section{CHAPTER 3}

practitioners do not judge the outcome of theory-based intervention to be beneficial and practicable, they can hardly be motivated to use theory, which impedes the collaboration with researchers.

Changing attitudes, perceived social expectations and outcome expectations requires arguments that are new to the participants, that are relevant and that are perceived as correct. Arguments should focus on the benefits of and barriers to collaboration. Awareness is often described as the first step in the change process and can confront niche members with differences in attitude, perceived social expectations and outcome expectations ${ }^{176,178}$. Multidisciplinary group discussions supply new arguments and new insights and may convince niche members of the benefits of collaboration. Social Cognitive Theory interventions are based on active learning, that is, learning by doing, which promotes performance and may change attitudes during the learning process. Correction of misinformation or incorrect actions and provision of incentives or assistance can reduce perceived barriers and can change attitudes, perceived norms and outcome expectations. Discussions of perceived social expectations might start from the norms that prevail within the other organization as regards the way work is usually carried out. Multidisciplinary group meetings to address issues of shared interest may adjust the content of joint public health proposals as well as the operational processes to create solutions and remove experienced barriers. This may result in affective support to gain social skills beyond people's own niche.

Behavioural capability means that if a person is to perform a specific behaviour, he must know what the behaviour is and how to perform it. Selfefficacy is someone's perception or judgement about accomplishing a certain level of performance. Looking beyond the boundaries of one's own niche and collaborating with other niches requires new capacities, and professionals must believe that they can acquire these capacities. According to Learning Theory and Social Cognitive Theory, interventions to build capacity and increase selfefficacy should make use of goal setting, active learning, reinforcement and guided practice $178,196,256$. Setting challenging but feasible goals stimulates people's performance, provided that they commit themselves to the goal, are able to achieve it and have access to organized feedback. It is important to provide training and guidance through various practical strategies, such as feedback by peer networking (one efficient form being an e-mail listserver to share information and assist each other)228,256; a training network and lectures to communicate new insights and to stimulate those who do not yet participate in the collaboration; individual in-service coaching after participation in master classes to learn to apply new knowledge and skills, especially to write reports intended for a readership beyond one's own niche. If people are to effectively engage in collaboration between research, policy and practice, they require a set of personal qualities, including enthusiasm, ambition, energy, patience, persistence, 
self-confidence, personal credibility, willingness to be flexible and take risks, commitment to learn from experience, and a sense of being engaged in meaningful projects and partnerships ${ }^{256}$. These qualities should be embedded in the training courses.

\section{OVERVIEW OF PRACTICAL STRATEGIES}

In this chapter we have tried to identify theory-based practical strategies that can be implemented to improve collaboration. In these practical strategies, the formal stakeholders decide on the outlines, after which management approves action plans relating to organizational development, climate, culture and capacity building. Such action plans can then be put into practice, with management guiding the implementation at institutional and individual level.

Preparing a collaborative effort requires practical strategies to bridge the gaps between the niches. The focus at the various system levels can differentiate between content and process. At the administrative level, the content of the public health problem in terms of general features is the starting point for collaboration, so practical strategies deal with the broad outlines of the content. The interpersonal process aspects are crucial, as coalitions will not be achieved between combating participants. At the institutional level, the collaboration process in itself needs to be worked on, with special focus on the organizational development plan. At this level, process factors are of crucial importance. At the individual level, the content of the public health problem and the collaboration process are tied together. For example, multidisciplinary group discussions will start with details of the content of the problem, which may result in improvement of the collaboration process as group members come to value each other's contributions, which in turn may stimulate affective ties, informal consultations and mutual respect.

Another essential aspect in relation to the policy cycle is the timing of the problem assessment. If a problem is to be addressed at the end of the policy cycle, when the local government has to show results for the previous period, the impact of practice and research on policy will be negligible, as the local government will not initiate new issues. However, if this is done just after local elections and the formation of a new local government, chances of success will be greater ${ }^{150}$. The timing of interconnections between the policy, research and practice cycles must be thoroughly considered to overcome the pacing problem discussed in chapter 2.

Given the actors and the focus in the collaboration process, the various practical strategies are summarized in table 3.1. This overview should not be interpreted as compulsory, but as a tool that can help decide on practical strategies that fit in with the actual state of collaboration between the niches. Niche 


\section{CHAPTER 3}

stakeholders and professionals may offer joint, sound judgements on the practical strategies they deem necessary and the extent to which they should be implemented, as well as those strategies that can be omitted. Implementation of these practical strategies is only one side of the matter. Another important aspect relating to the intention of the practical strategies is their quality ${ }^{188}$. For instance, using the practical strategy of role models might have an adverse effect if the wrong role model is selected. Or using the strategy of commitment planning might have adverse effects if the wrong key players are identified. Capacity building will fail if the content and the methods used in the training course are insufficiently tailored to the needs of the niche professionals. Therefore, the quality of the strategies is an essential precondition in the collaboration between policy, practice and research. The concept of quality is complex and includes effectiveness, relevance, reach, cost-benefit ratio, ethical justification, satisfaction among clients or society, appreciation by professionals and practical feasibility ${ }^{270}$. These output characteristics can only be achieved if certain requirements are met in designing and preparing the implementation of the practical strategies. Elaborating on quality management systems here is beyond the scope of this dissertation, but the collaboration between policy, practice and research should fit the operational methods that correspond to the concepts of the Dutch health care quality assurance policy. This policy is based on the model developed by the European Foundation for Quality Management, and hence called the EFQM model, while in Dutch it is known as the INK model (after the Instituut Nederlandse Kwaliteit) ${ }^{271}$. At present, the Dutch Harmonisation of Quality Review in Health Care and Welfare (HKZ), together with the health promotion sector, are working on a certification scheme for health promotion, applied to the practice of GGD work.

Table 3.1 Collaboration programme methods* and practical strategies** at high and low system levels

\begin{tabular}{|l|l|l|}
\hline $\begin{array}{l}\text { DETERMINANTS AT } \\
\text { ADMINISTRATIVE LEVEL }\end{array}$ & METHODS & PRACTICAL STRATEGIES \\
\hline $\begin{array}{l}\text { power } \\
\text { leadership }\end{array}$ & $\begin{array}{l}\text { policy advocacy } \\
\text { coalition } \\
\text { modelling } \\
\text { social or policy entrepre- } \\
\text { neurship }\end{array}$ & $\begin{array}{l}\text { advocacy } \\
\text { coalition building } \\
\text { sidestepping the formal proce- } \\
\text { dures, lobbying } \\
\text { agenda setting }\end{array}$ \\
\hline $\begin{array}{l}\text { Desources } \\
\text { INSTERMINANTS AT }\end{array}$ & negotiation \\
\hline $\begin{array}{l}\text { METHODS } \\
\text { Organizational development } \\
\text { focusing on climate, culture }\end{array}$ & organizational development & $\begin{array}{l}\text { diagnosis } \\
\text { action planning: activities and } \\
\text { interventions commitment }\end{array}$ \\
\hline
\end{tabular}




\begin{tabular}{|c|c|c|}
\hline $\begin{array}{l}\text { and capacity building in } \\
\text { relation to public health in } \\
\text { general or a specific se- } \\
\text { lected issue }\end{array}$ & & $\begin{array}{l}\text { intersectoral management struc- } \\
\text { ture } \\
\text { communication structure } \\
\text { evaluation } \\
\text { resources }\end{array}$ \\
\hline $\begin{array}{l}\text { climate of a learning organi- } \\
\text { zation }\end{array}$ & $\begin{array}{l}\text { goal setting } \\
\text { media portrayals } \\
\text { behavioural journalism }\end{array}$ & $\begin{array}{l}\text { mission statement } \\
\text { interviews with role models, PR }\end{array}$ \\
\hline $\begin{array}{l}\text { culture of shared values and } \\
\text { beliefs }\end{array}$ & $\begin{array}{l}\text { modelling (in particular by } \\
\text { the leader) } \\
\text { social support } \\
\text { increasing reciprocity }\end{array}$ & $\begin{array}{l}\text { internships } \\
\text { service delivery between niches } \\
\text { access to tools of other niches } \\
\text { formal and informal consultation } \\
\text { structures }\end{array}$ \\
\hline capacity building & $\begin{array}{l}\text { partnerships / group struc- } \\
\text { ture } \\
\text { enhancing experience \& } \\
\text { competence } \\
\text { removing social and envi- } \\
\text { ronmental barriers } \\
\text { increasing environmental } \\
\text { support }\end{array}$ & $\begin{array}{l}\text { diagnosis and feedback to staff } \\
\text { recruiting and retaining staff } \\
\text { professional development pro- } \\
\text { gramme } \\
\text { kick-off conference }\end{array}$ \\
\hline leadership & modelling & sidestepping the formal system \\
\hline $\begin{array}{l}\text { DETERMINANTS AT } \\
\text { INDIVIDUAL LEVEL }\end{array}$ & METHODS & PRACTICAL STRATEGIES \\
\hline knowledge & information & $\begin{array}{l}\text { diagnosis, feedback by (group) } \\
\text { discussion }\end{array}$ \\
\hline $\begin{array}{l}\text { attitude } \\
\text { perceived norms } \\
\text { outcome expectations }\end{array}$ & $\begin{array}{l}\text { arguments } \\
\text { active learning, direct experi- } \\
\quad \text { ence } \\
\text { shifting perspective } \\
\text { incentives, assistance }\end{array}$ & $\begin{array}{l}\text { group discussion in multidiscipli- } \\
\text { nary groups } \\
\text { learning by doing }\end{array}$ \\
\hline $\begin{array}{l}\text { behavioural capability } \\
\text { self-efficacy }\end{array}$ & $\begin{array}{l}\text { goal setting and feedback } \\
\text { social network } \\
\text { social support } \\
\text { active learning } \\
\text { reinforcement } \\
\text { guided practice }\end{array}$ & $\begin{array}{l}\text { detailed action plan, formats, } \\
\text { materials } \\
\text { feedback by peer networking } \\
\text { lectures and discussion } \\
\text { master classes with skills training } \\
\text { in-service coaching to write } \\
\text { reports or articles intended for } \\
\text { a readership beyond one's own } \\
\text { niche }\end{array}$ \\
\hline
\end{tabular}




\section{CHAPTER 3}

\section{SUMMARY}

In the previous chapters, we have stressed the need to develop evidence-based public health through collaboration between research, practice and policy. Next, we have explored the differences between the three niches involved in public health, including the unequal pacing of the work cycles, which impede collaboration. In the present chapter we have described a logic model that distinguishes the relevant determinants at each system level. We have presented a survey of theory-based practical strategies that may enhance opportunities for collaboration. The key aspect in this survey is the creation of a planned dialogue between policy, practice and research at the administrative, institutional and individual levels. We assume that a planned dialogue will contribute to a better understanding between niches, an increased intention to collaborate and a reduction of the disparities between the niches and the pacing problem. In the next chapter we evaluate this theory-based framework with the help of the opinions of experts from the field of public health practice, policy and research, to arrive at a more or less conclusive decision on practical strategies that can effectively support the collaboration between practice, policy and research. 


\section{Expert meeting}

\section{INTRODUCTION}

The research question this thesis tries to answer is that bridging the gaps between the three niches (policy, practice and research) will result in collaboration and in the long run in quality improvements and evidence-based public health practices and policies. How to build such bridges was the subject of chapter 3, where we proposed 30 practical strategies, based on theory, to achieve the desired interface. To find out whether these theory-based practical strategies correspond with the opinions of public health experts, an expert meeting was organized. The present chapter describes the design of the meeting with the experts, its results and how we reached consensus about the framework. Finally, it presents the framework of practical strategies which formed the starting point for the multiple-case study. This framework was used as a checklist to evaluate the six cases.

\section{DESIGN AND RESPONDENTS}

The goal of the expert meeting was to examine practical strategies for collaboration between policy, practice and research proposed by experts and to compare the degrees of priority they gave to these strategies with the theory-based practical strategies reported on in chapter 3.

Twenty-six Dutch experts from the domains of policy, practice and research were approached, using a snowball method. Eighteen expressed their willingness to participate (a response of 69\%), 15 of whom were present at the expert meeting and 3 - all from the policy domain - were interviewed individually because they were unable to attend. The main inclusion criterion was whether the person considered himself/herself an expert in the field of policy, practice or research, or combinations of these fields. Ages varied from 32 to 60 years, with equal numbers of men and women. All experts had a university degree, except one who had a degree in higher professional education; nine persons had a $\mathrm{PhD}$ degree. The respondents included policy makers (6, of whom 3 attended the expert meeting while 3 were individually interviewed), researchers (3), practitioners (4) and combinations (5). The experts represented 


\section{CHAPTER 4}

the following organizations: GGD (4 experts), municipal authorities (4 experts), the National Institute for Health Promotion and Disease Prevention (NIGZ, 2 experts), the Netherlands Organization for Health Research and Development (ZonMw, 1 expert), the Association of Care Organizations (Z-org, 1 expert), the Netherlands Organization for Applied Scientific Research (TNO, 1 expert), the Institute of Health Policy and Management of Rotterdam Erasmus University (Instituut Beleid en Management Gezondheidszorg, 1 expert), the Department of Health Promotion of Maastricht University (1 expert), the School of Politics and Public Administration of the Faculty of Law of the Tilburg University (1 expert), and the National Institute of Public Health and the Environment (RIVM, 2 experts). We had intended to involve the Dutch Health Care Inspectorate and the Ministry of Health, Welfare and Sport, but they were unable to send a representative. Policy makers were overrepresented among the group of non-responders (5 out of 8).

The group session lasted three hours and was led by the author of this thesis, who acted as moderator. The moderator followed a predetermined format, which involved a short introduction, followed by group discussions, a priority setting procedure, and provisional conclusions about the priorities. The meeting was tape-recorded and transcribed afterwards. Participants were asked to introduce themselves and to mention one important practical strategy to get the discussion going. The moderator gave a short introduction about the barriers between policy, practice and research which impede collaboration, and explained the aim of the meeting and the procedure. Thereafter, the group was split up into four smaller subgroups, each including representatives of the domain of policy, practice and research. Each subgroup had to conceive practical strategies that would contribute to collaboration. Next, the proposed strategies were summarized and categorized by the moderator, using three categories or system levels: the administrative level, i.e. the system level where the final decisions are made; the institutional level, i.e. the level of organizations which employ professionals; and the individual level, i.e. the individual practitioners, researchers and policy makers. As was described in chapter 3, practical strategies at the lowest system level may be added to the next higher system level, and these in turn to the highest system level (see chapter 3) to demonstrate the coherence between the levels.

Twenty-eight strategies emerged from the group discussions (see table 4.1, page 76); 7 strategies at the administrative level; 16 at the institutional level and 5 at the individual level. Strategies that were not mentioned by the subgroups but had been included in the theory-based framework were discussed and subsequently rejected or accepted by the experts. This concerned two strategies (intersectoral management and staffing policy / recruitment policy), which are underlined in table 4.1. A few more strategies were added later on as a result of further discussions during the meeting, or emerged from the interviews with 
individual participants; these are shown in italics. The numbered and underlined strategies were prioritized by the four subgroups (the ones in italics were not, as they were added to the list after the meeting). Finally, the four subgroups had to negotiate the priorities to be assigned to the practical strategies at each system level. They were asked to assign priority levels to half of the number of proposed strategies, to be rounded off if there were an odd number of strategies. Priority-setting was done by giving the highest score to the practical strategy with the highest priority. Participants were allowed to select 4 of the 7 strategies at the administrative level ( $\max$ score 4 , min score 1); 9 of the 17 at the institutional level ( $\max$ score 9 , min score 1 ) and 3 of the 5 at the individual level (max score 3 , min score 1). The scores of the subgroups were added up which resulted in a prioritized list of strategies to support collaboration. The practical strategies given high priority are shown in bold in table 4.1. A priority rank of 1 in the table means that the highest priority was given. Equal ranks refer to equal scores. The negotiations about priority-setting within the subgroups were characterized as involving compromise, mutual respect and making a deal. Whether this reflects the real-life negotiations is unclear.

After the expert meeting, the proposed strategies and priorities were compared with those distinguished within the theory-based framework (see chapter 3, table 3.1). The theory-based framework was used as a basic framework into which we tried to fit the experts' opinions. Because the experts had not defined their proposed practical strategies in detail at the meeting, the categories to which they were assigned were based on interpretation of the discussions at the meeting, and checked afterwards using the transcript of the meeting. Finally, to clarify the interpretation, each practical strategy was more precisely defined, making use of the concepts mentioned by the experts during the meeting and recorded in the transcript. The procedure of fitting the experts' opinions within the categories of the theory-based framework (table 4.1) was based on these final descriptions of practical strategies, which were related to the original determinants (appendix A, page 78). This procedure was supervised by three other persons who had attended the expert meeting, to avoid subjective judgements by the author.

\section{RESULTS}

The results of the group debates and the scores they awarded to the various proposed strategies are summarized in table 4.1. A comparison of the practical strategies proposed at the expert meeting and the theory-based framework of practical strategies showed that the experts' opinions generally matched the theory-based framework. Most of the differences could be solved by combining theory and expert opinion on the basis of concepts the experts mentioned dur- 


\section{CHAPTER 4}

ing the meeting. These combinations were included in the final descriptions of strategies. This allowed us to reconcile theory-based and expert-based strategies.

There was agreement on the importance of brokers, or social and policy entrepreneurs, at the highest system level. Collaboration as the guiding principle was given high priority, which reflects the importance of consulting the other domains. Since this principle has to be advocated at the highest system level, the strategy of advocacy was chosen as the appropriate working method to attain the outcome in terms of collaboration. Collaboration as the leading principle was subsequently included in the description of the practical strategy of advocacy. The practical strategy of coalition building includes elements like systematic involvement of partners, defining final outcomes and a common goal, as has been included in the description. The experts also mentioned the practical strategy of building networks, explicitly recommending its use at each system level.

At the institutional level, the highest priority was given to competence, staffing policy and recruitment policy. The expert meeting did not discuss the type of competence required, though this is of course an interesting question for the development of collaboration. Experts attached great significance to communication, as their second priority, and confirmed the urgency of understanding each other's language, mission and goals. One concept often mentioned during the meeting and in the interviews was that of establishing dialogues. The relevance of incentives was recognized, as well as the need for resource allocation, reciprocity, a mission statement, an intersectoral management structure and organizational development.

At the individual level, the main practical strategy was education. The moderator suggested to interpret this proposed strategy in a broad sense, including various educational methods such as multidisciplinary groups, learning by doing, master classes, feedback by peer networking, and lectures. One of the interviewed experts suggested an educational method based on the concept of the 'reflective practitioner' ${ }^{142,272}$ as an effective strategy to create convergence among professionals.

There were some remarkable differences between the theory-based framework and the experts' opinion. One practical strategy, viz. differentiation of the concept of evidence, did not fit within the theory-based framework categories. We considered this aspect to be the final outcome of collaboration. Another strategy discussed by the experts, viz. performance indicators for collaboration to ensure accountability to management, was judged as highly significant but was also lacking from the theoretical framework. So far, it has not been common practice to include performance indicators of collaboration in the regular institutional lists of performance indicators. Practitioners, researchers nor policy makers are rewarded for their efforts to achieve collaboration. 
The theory-based strategy of organizing a professional development programme involves personal career plans and the wish to accept new challenges. Evaluation and monitoring of the actions undertaken to meet these challenges requires performance indicators. Therefore, the professional development programme strategy was widened to include performance indicators and professional development. Collaboration can be regarded as a new challenge to professionals, which requires a development programme and performance indicators for its evaluation.

As the experts at the meeting had been asked not to do any preparatory work, there is of course less coherence between the practical strategies proposed at this meeting than in the theory-based framework, which was constructed after a thorough literature research. Nevertheless, most of the theorybased strategies were actually mentioned by the experts. We have included each proposed strategy in only one of the system levels of the theoretical framework. For example, the experts ascribed great impact to the role of brokers, without specifying the system level. We decided to classify the role of brokers at the administrative level, while the experts may have intended this role at both the administrative and institutional levels. The decision to ask the experts to assign priorities per system level limited the priority-setting to the selected level. Nevertheless, we conclude that the theory-based framework, supplemented with the opinions of experts, resulted in an acceptable framework which could be used as the starting point for the multiple-case study.

\section{CONCLUSION}

The systematic process of developing practical strategies based on theory yielded thirty strategies. These thirty strategies were evaluated by eighteen experts from the field of practice, policy, research or combinations. No one disputed that there is a need for collaboration between the domains of public health policy, practice and research. The group agreed that there are multiple practical strategies to promote such collaboration. Although the practical strategies mentioned by the experts and those based on theory were described differently, the meanings each were given highly corresponded. Discrepancies were solved by redefining the practical strategy by matching the meaning of experts and theory. The overview of redefined strategies was used as a checklist to evaluate the multiple-case study as is described in chapter 5 . 


\section{CHAPTER 4}

Table 4.1 Results of the expert meeting, categorized in terms of the theory-based framework of practical strategies

\begin{tabular}{|c|c|c|c|}
\hline $\begin{array}{l}\text { EXPERT MEETING: } \\
\text { PRACTICAL STRATEGIES AT } \\
\text { THE ADMINISTRATIVE LEVEL }\end{array}$ & $\begin{array}{l}\text { SUM } \\
\text { SCORE }\end{array}$ & $\begin{array}{l}\text { PRIORITY } \\
\text { RANKING }\end{array}$ & $\begin{array}{l}\text { THEORY-BASED FRAMEWORK: } \\
\text { PRACTICAL STRATEGIES AT THE } \\
\text { ADMINISTRATIVE LEVEL }\end{array}$ \\
\hline $\begin{array}{l}\text { 1. advocacy on collaboration as } \\
\text { the guiding principle }\end{array}$ & 6 & 2 & advocacy \\
\hline $\begin{array}{l}\text { 2. assessment of final outcomes } \\
\text { of collaboration }\end{array}$ & 5 & 3 & coalition building \\
\hline $\begin{array}{l}\text { 3. systematic involvement of } \\
\text { partners }\end{array}$ & 4 & & \\
\hline 4. networks & 5 & 3 & \\
\hline 5. common goals & & & \\
\hline 6. brokers & 10 & 1 & $\begin{array}{l}\text { sidestepping the formal system, } \\
\text { lobbying } \\
\text { agenda setting }\end{array}$ \\
\hline 7. resources & 7 & & resource negotiations \\
\hline $\begin{array}{l}\text { EXPERT MEETING: } \\
\text { PRACTICAL STRATEGIES AT } \\
\text { THE INSTTTUTIONAL LEVEL }\end{array}$ & & & $\begin{array}{l}\text { THEORY-BASED FRAMEWORK: } \\
\text { PRACTICAL STRATEGIES AT THE } \\
\text { INSTITUTIONAL LEVEL }\end{array}$ \\
\hline $\begin{array}{l}\text { 8. exploration, focusing on as- } \\
\text { pects of relations between } \\
\text { partners }\end{array}$ & 6 & & \\
\hline \multirow[t]{2}{*}{ 9. quality criteria } & 7 & & \\
\hline & & & diagnosis for OD* \\
\hline 10. organizational development & 8 & 8 & action planning for OD \\
\hline 11. incentives & 14 & 3 & $\begin{array}{l}\text { activities and interventions for } \\
\text { OD }\end{array}$ \\
\hline 12. intention to collaborate & 0 & & commitment \\
\hline intersectoral management & 10 & 7 & $\begin{array}{l}\text { intersectoral management struc- } \\
\text { ture }\end{array}$ \\
\hline \multirow[t]{2}{*}{$\begin{array}{l}\text { 13. communication } \\
\text { dialogue }\end{array}$} & 15 & 2 & communication structure \\
\hline & & & evaluation \\
\hline $\begin{array}{l}\text { 14. steering and control by re- } \\
\text { source allocation }\end{array}$ & 13 & 4 & resources \\
\hline 15. mission of the organization & 11 & 6 & mission statement \\
\hline \multirow[t]{2}{*}{ 16. reciprocity } & 13 & 4 & \\
\hline & & & interviews with role models \\
\hline \multirow[t]{2}{*}{ 17. internships } & 6 & & internships \\
\hline & & & $\begin{array}{l}\text { rendering services between } \\
\text { niches } \\
\text { access to tools of other niches }\end{array}$ \\
\hline
\end{tabular}




\begin{tabular}{|c|c|c|c|}
\hline $\begin{array}{l}\text { 18. formal and informal consulta- } \\
\text { tions }\end{array}$ & 8 & 8 & $\begin{array}{l}\text { formal and informal consulta- } \\
\text { tion structures }\end{array}$ \\
\hline 19. networks & 3 & & \\
\hline $\begin{array}{l}\text { 20. competence } \\
\text { staffing policy / recruitment } \\
\text { policy }\end{array}$ & 20 & 1 & $\begin{array}{l}\text { diagnosis and feedback to staff } \\
\text { concerning capacity } \\
\text { recruiting and retaining staff }\end{array}$ \\
\hline 21. training & 0 & & $\begin{array}{l}\text { professional development pro- } \\
\text { gramme }\end{array}$ \\
\hline \multirow[t]{2}{*}{ 22. conference, congress } & 0 & & kick-off conference \\
\hline & & & sidestepping the formal system \\
\hline \multicolumn{4}{|l|}{$\begin{array}{l}\text { 23. widening the concept of evi- } \\
\text { dence }\end{array}$} \\
\hline \multicolumn{4}{|l|}{$\begin{array}{l}\text { performance indicators on } \\
\text { collaboration to ensure ac- } \\
\text { countability to management }\end{array}$} \\
\hline $\begin{array}{l}\text { EXPERT MEETING: } \\
\text { PRACTICAL STRATEGIES AT } \\
\text { THE INDIVIDUAL LEVEL }\end{array}$ & & & $\begin{array}{l}\text { THEORY-BASED FRAMEWORK: } \\
\text { PRACTICAL STRATEGIES AT THE } \\
\text { INDIVIDUAL LEVEL }\end{array}$ \\
\hline 24. problem diagnosis & 6 & 1 & $\begin{array}{l}\text { diagnosis and feedback by } \\
\text { (group) discussion }\end{array}$ \\
\hline 25. attitude & 0 & & \\
\hline 26. education (various methods) & 2 & & $\begin{array}{l}\text { group discussion in multidisci- } \\
\text { plinary groups } \\
\text { learning by doing } \\
\text { feedback by peer networking } \\
\text { lectures and discussion } \\
\text { master classes with skills training }\end{array}$ \\
\hline $\begin{array}{l}\text { 27. division of roles between } \\
\text { partners }\end{array}$ & 0 & & $\begin{array}{l}\text { detailed action plan, formats, } \\
\text { materials }\end{array}$ \\
\hline \multicolumn{4}{|l|}{ reflective practitioner } \\
\hline $\begin{array}{l}\text { 28. publications beyond one's own } \\
\text { domain }\end{array}$ & 4 & 2 & $\begin{array}{l}\text { in-service coaching to write } \\
\text { reports or articles intended for } \\
\text { a readership beyond one's } \\
\text { own niche }\end{array}$ \\
\hline
\end{tabular}

underlined $=$ strategies from the theory-based framework, added and accepted during the expert meeting;

bold = assessed as priority during the expert meeting;

italics $=$ added after the expert meeting, based on discussion or individual interviews;

$* \mathrm{OD}=$ Organizational Development 


\section{CHAPTER 4}

\section{Appendix A}

Descriptions of practical strategies at the different system levels, based on theory and experts' opinions

DETERMINANTS AT
THE ADMINISTRA-
TIVE LEVEL

Power
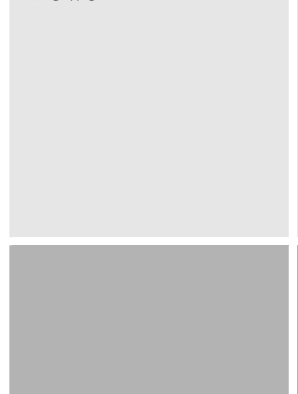

Leadership
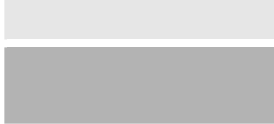

Resources

\section{DETERMINANTS AT THE INSTITUTIONAL LEVEL}
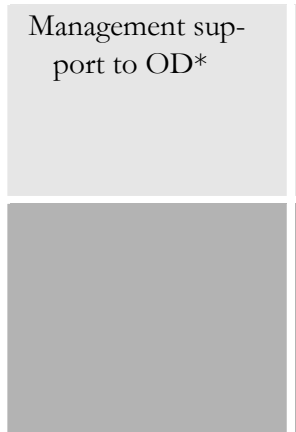

action planning: activities and interventions

commitment agement structure GIES AT THE ADMINISTRATIVE LEVEL

PRACTICAL STRATETIONAL LEVEL

diagnosis for OD* intersectoral man-

\section{DEFINITION}

consultation, information, persuasion, negotiation or coercion, focusing on collaboration between research, practice and policy as the guiding principle to influence the political or policy agenda in order to achieve a common goal related to a particular problem/content

a formal agreement to organize and systematically involve individuals representing various organizations who work together to achieve a common goal

consultation activities by at least one person/leader who operates as a broker or social entrepreneur to influence people's willingness to collaborate

items being formally put on the local political agenda

formal agreement about the resources needed

exploration of the collaboration process, investigating mission, goals, climate, culture and capacity, intended outcomes in terms of collaboration and willingness to take action within and beyond institutions

road map for the collaboration process, citing consecutively planned activities and interventions relating to climate, culture and capacity, which must be undertaken to achieve the desired collaboration outcomes and which is approved by the management

identification of key players whose commitment to collaboration is needed, and formulation of a strategy to gain support

change-management structure: a chief executive, a project manager, formal managers, steering committee, working groups 


\begin{tabular}{|c|c|c|}
\hline & $\begin{array}{l}\text { communication } \\
\text { structure }\end{array}$ & $\begin{array}{l}\text { an overview of the communication structure, } \\
\text { activities and planning between and beyond } \\
\text { the partners }\end{array}$ \\
\hline & evaluation & $\begin{array}{l}\text { systematic assessment of the effects of activi- } \\
\text { ties and interventions, focusing on the col- } \\
\text { laboration process indicators and intended } \\
\text { collaboration outcomes }\end{array}$ \\
\hline & resources & $\begin{array}{l}\text { steering and control by resource allocation; } \\
\text { allocated time and money and appointed } \\
\text { staff, access to services of other niches to } \\
\text { improve knowledge transfer, earmarked } \\
\text { budgets for capacity building }\end{array}$ \\
\hline \multirow[t]{2}{*}{$\begin{array}{l}\text { Climate of a learn- } \\
\text { ing organization }\end{array}$} & mission statement & $\begin{array}{l}\text { explicit mission statement tailored to the } \\
\text { organizational climate, including the intention } \\
\text { to be a learning organization by acknowledg- } \\
\text { ing the reciprocal relations between policy, } \\
\text { practice and research and being prepared to } \\
\text { collaborate in terms of content and process }\end{array}$ \\
\hline & $\begin{array}{l}\text { interviews with role } \\
\text { models }\end{array}$ & $\begin{array}{l}\text { printed and published interviews or PR } \\
\text { activities within or beyond the collaboration } \\
\text { structure, relating to the advantages of a } \\
\text { collaborative learning organization which } \\
\text { operates in partnership with other organiza- } \\
\text { tions }\end{array}$ \\
\hline \multirow[t]{3}{*}{$\begin{array}{l}\text { Culture of shared } \\
\text { values and beliefs }\end{array}$} & internships & $\begin{array}{l}\text { internships or secondments between the } \\
\text { collaborating institutions, to establish links } \\
\text { between the cultures of the various niches } \\
\text { and improve knowledge transfer }\end{array}$ \\
\hline & $\begin{array}{l}\text { rendering services } \\
\text { between niches, } \\
\text { access to tools of } \\
\text { other niches }\end{array}$ & $\begin{array}{l}\text { services are exchanged and access to tools is } \\
\text { arranged without barriers as part of the work- } \\
\text { ing culture }\end{array}$ \\
\hline & $\begin{array}{l}\text { formal and informal } \\
\text { consultation struc- } \\
\text { tures }\end{array}$ & $\begin{array}{l}\text { permanent working groups in which mem- } \\
\text { bers can easily and informally contact each } \\
\text { other for advice and knowledge transfer }\end{array}$ \\
\hline \multirow[t]{3}{*}{ Capacity building } & $\begin{array}{l}\text { diagnosis and feed- } \\
\text { back to staff concern- } \\
\text { ing capacity }\end{array}$ & $\begin{array}{l}\text { intermittent assessments of available and } \\
\text { required capacity, with feedback to the man- } \\
\text { agement of the organizations or the project } \\
\text { management }\end{array}$ \\
\hline & $\begin{array}{l}\text { recruiting and retain- } \\
\text { ing staff }\end{array}$ & $\begin{array}{l}\text { recruitment of extra staff or retention of staff } \\
\text { qualified to support the collaboration }\end{array}$ \\
\hline & $\begin{array}{l}\text { performance indica- } \\
\text { tors and professional } \\
\text { development }\end{array}$ & $\begin{array}{l}\text { accountability of staff to management in } \\
\text { terms of performance indicators for collabo- } \\
\text { ration, combined with professional develop- } \\
\text { ment to stimulate professionals to collaborate } \\
\text { with partners, supported by incentives and } \\
\text { approved by management }\end{array}$ \\
\hline
\end{tabular}




\begin{tabular}{|c|c|c|}
\hline & kick-off conference & $\begin{array}{l}\text { organizing a kick-off conference in which the } \\
\text { content and process of collaboration are } \\
\text { communicated formally and informally }\end{array}$ \\
\hline Leadership & $\begin{array}{l}\text { sidestepping the } \\
\text { formal system }\end{array}$ & $\begin{array}{l}\text { consultation activities by at least one per- } \\
\text { son/leader with hierarchic support or sup- } \\
\text { port from a social or policy entrepreneur, to } \\
\text { influence organizational development at } \\
\text { management level and to communicate about } \\
\text { progress with all partners }\end{array}$ \\
\hline $\begin{array}{l}\text { DETERMINANTS AT } \\
\text { THE INDIVIDUAL } \\
\text { LEVEL }\end{array}$ & $\begin{array}{l}\text { PRACTICAL STRATE- } \\
\text { GIES AT THE INDIVID- } \\
\text { UAL LEVEL }\end{array}$ & DEFINITION \\
\hline Lack of knowledge & $\begin{array}{l}\text { diagnosis and feed- } \\
\text { back by project leader }\end{array}$ & $\begin{array}{l}\text { intermittent assessment of the available and } \\
\text { required knowledge and expertise, with } \\
\text { feedback to the project leader }\end{array}$ \\
\hline \multirow[t]{2}{*}{$\begin{array}{l}\text { Attitude } \\
\text { Perceived norms } \\
\text { Outcome expecta- } \\
\text { tions }\end{array}$} & $\begin{array}{l}\text { group discussion in } \\
\text { multidisciplinary } \\
\text { groups }\end{array}$ & $\begin{array}{l}\text { formal and structured group discussions } \\
\text { between the partners to exchange arguments, } \\
\text { experiences etc., from different perspec- } \\
\text { tives/niches }\end{array}$ \\
\hline & learning by doing & $\begin{array}{l}\text { task assignment by the project leader to } \\
\text { group members and feedback on the imple- } \\
\text { mentation of these new tasks }\end{array}$ \\
\hline \multirow[t]{5}{*}{$\begin{array}{l}\text { Behavioural capa- } \\
\text { bility } \\
\text { Self-efficacy }\end{array}$} & $\begin{array}{l}\text { detailed action plan, } \\
\text { formats, materials }\end{array}$ & $\begin{array}{l}\text { written materials or verbal instructions on } \\
\text { what to do, how, where and when, including } \\
\text { division of tasks between partners }\end{array}$ \\
\hline & $\begin{array}{l}\text { feedback by peer } \\
\text { networking }\end{array}$ & $\begin{array}{l}\text { informal networks between like-minded } \\
\text { professionals (peers) to enhance capability } \\
\text { and self-efficacy }\end{array}$ \\
\hline & $\begin{array}{l}\text { lectures and discus- } \\
\text { sions }\end{array}$ & $\begin{array}{l}\text { lectures and discussions to explore future } \\
\text { implementation and improvements beyond } \\
\text { the current situation }\end{array}$ \\
\hline & $\begin{array}{l}\text { master classes with } \\
\text { skills training }\end{array}$ & $\begin{array}{l}\text { master classes offering skills training to teach } \\
\text { staff to apply new working methods }\end{array}$ \\
\hline & $\begin{array}{l}\text { in-service coaching to } \\
\text { write reports intended } \\
\text { for a readership } \\
\text { beyond one's own } \\
\text { niche }\end{array}$ & $\begin{array}{l}\text { on-the job coaching by a qualified profes- } \\
\text { sional (a kind of apprentice system) as re- } \\
\text { gards in-service coaching to write reports or } \\
\text { articles intended for a readership beyond } \\
\text { one's own niche to promote knowledge } \\
\text { transfer }\end{array}$ \\
\hline
\end{tabular}

* $\mathrm{OD}=$ Organizational Development 


\section{Multiple-case study}

\section{OUTLINE}

This chapter examines the nature and extent of the application of the practical strategies outlined in the previous chapters in six different public health cases. A case is defined here as an intended collaboration between the policy, practice and research domains (the niches) concerning a specific public health subject. To explore the phenomenon of collaboration between the public health niches, the nature and extent of the application of practical strategies for collaboration have been studied for each individual case. It was expected that the findings might collectively result in more generalizable propositions to promote collaboration.

The cases are derived from the author's experience in the field of public health during the last eighteen years. Each case concerns a public health research question with practical and policy implications. Therefore, collaboration was obviously intended. The enclosed articles communicate the empirical findings on the object of study. These articles as such give little or no information on the general research question of this dissertation. Since what we can learn from the case studies goes beyond the information provided in the articles integrated in this chapter, each case is introduced by a summary indicating the wider scope of the subject of study, and the goal of the collaboration. This is then followed by the article on that specific case, as it was published or submitted. In addition to the information presented in the published and submitted articles, other data sources have been used retrospectively, such as annual reports, evaluation reports, official minutes of meetings and direct observations to evaluate the application of the thirty practical strategies.

Our hypothesis was that the application of various practical strategies at each level would contribute first to collaboration and next to evidence-based public health, and we comment on this hypothesis for each of the cases. Rather than as a result of exhaustive scientific research, this multiple-case study should be regarded as an attempt to learn from past experiences in bridging the gaps between the niches of policy, practice and research. As we described in chapter 3 , each system level incorporates the practical strategies at all the lower system levels. For example, the practical strategy of advocacy at the administrative level requires practical strategies at the individual level of administrators, such as 


\section{CHAPTER 5}

learning by doing. As the strategies described at each level are the most important ones, only these strategies have been evaluated here.

For each case study, a describtion is given of facts and findings that influenced the evaluation of the application of practical strategies. The overall evaluative assessment is marked in parentheses; for example, [advocacy +] means that the practical strategy of advocacy was applied in that particular case, whereas [advocacy -] means that it was not applied. Finally, all evaluative assessments on the six cases have been summarized in table 5.1 (see pages 220221). Based on these evaluations, we have tried to formulate general conclusions with regard to the practical strategies, while at the same time doing justice to certain unique circumstances.

\section{THE CASES}

The case studies included in this dissertation are representative examples of public health problems and offer clear illustrations of public health problems in general. Together, they address each phase of the PRECEDE-PROCEED model by Green and Kreuter ${ }^{177}$ and the Intervention Mapping model by Bartholomew and colleagues ${ }^{178}$. The following cases are presented here:

- Estimating the size of the population of problem youths in the Dutch town of Maastricht, using Frank-Snijders estimators. The project was implemented between 1994 and 1998.

- Sexually transmitted diseases in Limburg in 1997; prevalence according to a survey among general practitioners, specialists and microbiology laboratories. The project was implemented between 1996 and 1998.

- Effects on problem gambling of extended opening hours of gambling centres. The project was discontinuously implemented during the 19911992 and 1999-2000 periods.

- A systematic approach to hepatitis B vaccination programmes for risk groups: a literature study. Implementation between 1998 and 2006 and continued thereafter.

- The tailored 'Schoolbeat' programme: new concepts for health promotion in schools in the Netherlands. The project was implemented between 2000 and 2006, and continued thereafter.

- Continuation processes for a successful preventive intervention: Heartbeat 2. The concept of sustainability and the use of outcome indicators. The community and high-risk approaches were implemented during the 19982004 period, and the high-risk approach continued since 2004.

Cases 1 and 2 related to epidemiological analysis, which can be interpreted as an objective assessment of the needs of specific groups which might have policy and practice consequences. Case 3 studied the effects of environmental factors 
that may influence health policy. Cases 4 and 5 focused on the preparation stage of interventions and stress the need for a systematic approach to organize practice and decide on policy. Finally, case 6 examined the factors that are relevant for the continuation and sustainability of a successful pilot programme.

\section{ANALYSIS}

To be able to draw conclusions about the contributions of practical strategies to collaboration, each practical strategy was assessed on the basis of the theoretical framework presented in chapter 3 and the results of the expert meeting reported in chapter 4 . The detailed descriptions of the strategies are shown in appendix A of chapter 4 . The evaluations of the application of practical strategies cover the total implementation period of each of the programmes examined in the case studies. Some practical strategies may extinguish over time, while others are reinforced. Since collaboration is a dynamic, iterative process which cannot be defined in detailed blueprints, we assessed the implementation of practical strategies over time in general terms, varying from intensive/good implementation $(+)$, mixed implementation $(+-)$, to no implementation $(-)$. In doubtful cases, we studied the field documents. Ideally, the collaboration in these cases should involve partners from practice, research and policy at the administrative and institutional levels. If only two of the three parties collaborated, our assessment was 'mixed implementation' (+-). At the individual level, the implementation of practical strategies might be delegated to one or two of the three partners, and so we assigned a judgement of 'good implementation' if the delegation agreements and task assignments for a practical strategy had been correctly implemented.

While assessing the cases, it sometimes proved rather difficult to distinguish between practical strategies and interventions. To recapitulate the definitions: a practical strategy is a way of organizing and operationalizing the theoretical method, and relates to the question of 'how to do' in a certain context with a specific group of people. An intervention is a set of sequentially planned actions or events intended to help achieve the goals that have been set, and concerns the question 'what to do'. The assessments of the six cases were based on the 'how to do' question, or in other words: how collaboration was promoted.

For each case, an overall sum score for the use of practical strategies was calculated by converting the assessments into numbers $(2$ for a $+; 1$ for a +and 0 for a - ). All practical strategies were given equal weight, as we did not have any indications that certain practical strategies had a stronger positive or negative impact on collaboration than others. The relationship between the overall score and successful collaboration is obviously not simply linear. Merely 


\section{CHAPTER 5}

summarizing superficially across the different cases by itself tells us little, though the numbers may make it easier to see whether the extent or intensity with which practical strategies are applied can predict the final outcome in terms of collaboration. However, we have to look carefully at the complex configuration of processes within each case, and understand the local dynamics, before we can see patterns that transcend the practical strategies in particular cases and allow general conclusions to be drawn ${ }^{135}$.

\section{THE RISK OF SUBJECTIVITY}

The assessment procedure was quite sensitive to subjectivity, as it was carried out by one person, viz. the author of this dissertation. Since this represented a serious threat to the validity of the study, I asked two independent judges to review the Schoolbeat case (case 5) and the Heartbeat case (case 6). The percentage of agreement between the two reviewers about the application of the 30 practical strategies was $67 \%$ for case 5 and $77 \%$ for case 6 , which was deemed acceptable. The levels of agreement on the sum scores were $94 \%$ and $98 \%$, respectively, and about the same for the subtotal per system level.

It should be kept in mind that the assessment procedure was carried out by professionals who work in the field of practice, i.e. the GGD (the Regional Public Health Service), as they might unconsciously attach greater value to strategies that are relevant to practitioners. 
MULTIPLE CASE STUDY

\subsection{CASE 1. INTRODUCTION}

\section{Estimating the size of the population of problem youths in the Dutch town of Maastricht, using Frank-Snijders estimators}

The goal of collaboration in this case was to obtain evidence-based results from the research and practice work cycles that could be applied in the policy work cycle for the purpose of local youth policy. The study was part of a wider investigation of the numbers and characteristics of problem youths, the use of youth health care among problem youths, and the effect of the early detection methods applied by the GGD's Youth Health Care service ${ }^{273}$. The GGD was asked by the Maastricht municipal authorities to assess the size of the population of problem youths, both those who were known and those who were unknown to the youth health care services. Registration systems of the clients of six youth health care organisations and mental health care services were linked by capture-recapture methods to estimate the overlap, that is, the number of clients using more than one health care service. The percentage of problem youths making use of health care or welfare services was estimated at $13 \%$. The overlap between the six services was negligible. The number of problem youths not receiving any care was estimated by a one-wave snowball procedure to be 2.5 to $3 \%$ of the Maastricht youths aged 12-24 years, as described in the article included below. Personal in-depth interviews were organized with 80 problem youths, identified by the street corner workers, asking them about the situation at home and at school, the way they spent their leisure time, their financial situation, their friends, drug use, quarrels and fights, contacts with the police or the judiciary, self-reported health and well-being. The same interviews were held with a control group of 80 youths, recruited at random in the street. Out of a total of 19 problems, the mean number mentioned by the control group was 2, against 9 amongst the problem youths; this difference was highly significant. The Youth Health Care records of the problem youths and the controls were screened over a maximum of 10 years to determine whether, in retrospect, signals could have been identified during the regular Youth Health Care examinations. Academic achievements, concentration, behavioural and emotional problems differed significantly between the groups. Fifty percent of the current problem youths had been identified through previous youth health care examinations. Finally, a meeting was organized with problem youths themselves to discuss the provisional recommendations and their viewpoints.

Recommendations were made to the policy makers on aspects such as a youth monitoring system, school health profiles, case managers for parents who experience problems raising their children, monitoring methods for school attendance and learning problems, improvement of school health promotion 


\section{CHAPTER 5}

activities, strengthening the welfare system, community participation by youths, and, last but not least, improved chances to find work, as youth unemployment was high, especially among the lower socio-economic groups ${ }^{274}$. 
Published (in Dutch) in Tijdschrift voor Gezondheidswetenschappen 1998;76:220-5

Estimating the size of the population of problem youths in the Dutch town of Maastricht, using Frank-Snijders estimators

Maria Jansen, MSc, coordinator Health Promotion and Education Division, GGD Zuid Limburg

Marinus Spreen, Assistant Professor at the Department of Statistics \& Measurement Theory, Groningen University (ICS, Rijksuniversiteit Groningen) 


\title{
CHAPTER 5
}

\begin{abstract}
A survey among the population of young people with behavioural problems in the Dutch town of Maastricht tried to estimate the size of the population, consisting of problem youths who were not known as such to youth care services. The municipal authorities were interested in their numbers as they wanted to use these data in developing local youth policy. Since problem youths constitute a so-called hidden population, we opted for an adjusted single-wave snowball sampling procedure. We successfully assembled a random initial sample, and used the Frank-Snijders estimators to calculate the size of the population of problem youths in Maastricht. The findings suggest that the town has 300-500 problem youths of Dutch ethnic origin in the 12-24 year age bracket. This corresponds to $2-3 \%$ of the Maastricht population in this age bracket. The method we used proved to be a valuable alternative option to assess the size of such a hidden population. On the other hand, it proved very difficult to gain access to the population of youngsters from ethnic minorities.
\end{abstract}

Key words: problem youths, population size estimates, 'hidden population', atrisk youngsters

\section{INTRODUCTION}

Recent media stories and reports from youth care services claim that the number of young people in the Netherlands who are in danger of developing serious social problems is rising. ${ }^{1}$ Alcohol and substance use among young people is on the rise, the number of youths arrested for assault, intimidation, manslaughter and murder has doubled since 1980, while the number of cases of violent robberies has tripled since 1980, ever more children are involved in parental divorce situations, and the number of reports of child abuse is increasing. ${ }^{1-4}$ Nationally, the proportion of problem youths has been estimated at $15 \% .^{5-9}$ Although some of these youths are clients of youth care services, many are not. The number of those not registered with such services is unknown, and these youths form a so-called hidden population. The Mastricht municipal authorities commissioned the regional public health service (GGD) to assess the size of this population, as they need to develop policies for these issues. ${ }^{10}$

The question the present study tried to answer was: how large is the population of problem youths who are not known as such to the youth care services (the 'hidden population')? 


\section{METHODS}

\section{Selection criteria for the target group}

Problems among young people are usually reflected in their behaviours, which can be divided into externalised and internalised problem behaviours. Internalised problem behaviours, also referred to as emotional problems, include inhibition and withdrawal, loneliness, eating disorders, depression, sense of powerlessness and suicidal thoughts; these behaviours appear to be more common among girls than boys. ${ }^{7}$ Externalised problem behaviours, also referred to as behavioural problems, are those behaviours that are visible to the people around the youths, and include aggression, truancy, vandalism, excessive use of stimulants and delinquency. This type of problem behaviour appears to be more common among boys than girls. $3,7,12$ The present study focused on externalised problem behaviours, as these behaviours inconvenience other people. The following selection criteria were used to define the target group:

- living in Maastricht;

- $\quad$ aged $12-24$ years;

- belonging to the group of young people exhibiting externalised problem behaviour, that is, showing one or more of the following behaviours:

a. irregular attendance at school, i.e., frequent truancy, being suspended or expelled from school;

b. excessive use of soft drugs and alcohol and/or any use of hard drugs like heroin or cocaine;

c. problems at home causing them to run away from home or live on the streets (homelessness, vagrancy);

d. frequent shoplifting and more serious crimes;

e. frequent extreme aggressiveness, as indicated by regular quarrels and fights.

\section{Single-wave snowball sampling technique}

Problem youths not registered with youth care services form a so-called hidden population, that is, a population whose members are difficult to approach or locate, or whose membership is difficult to determine. ${ }^{13}$ This means that no sampling frame is available. A potential alternative sampling frame might be obtained by using a random sample of the total population of the town aged between 12 and 24 years and selecting the problem youths within this sample. However, this approach turned out to be too expensive relative to the information that would be obtained. In addition, such a sampling procedure would result in serious selection bias, as homeless people, vagrants, truants, school dropouts etc. would be less likely to participate. We therefore opted for a sin- 


\section{CHAPTER 5}

gle-wave snowball sampling technique, a method commonly applied in studies into hidden populations. ${ }^{14,15}$

A single-wave snowball sample is obtained by taking a random sample of people from a network and then asking the people thus selected to name their contacts within the network. Theoretically, the use of the single-wave snowball sampling technique in the present study would require a random initial network sample and the existence of relations between the problem youths. The first of these preconditions could not be met in our study in Maastricht, as there was no suitable network sampling frame. The second requirement assumes that the problem youths form a network within which the behaviour to be researched in this case the externalised problem behaviour defined above - is the factor binding the target group together. ${ }^{16}$ This assumption seemed justified for problem youths who engage in truancy, vandalism, excessive use of stimulants, aggressive behaviour and petty or more serious crimes, based on the many media reports about youngsters 'hanging out' at favourite sites, as well as about gangs of youths and groups of young people causing a nuisance. This means that problem behaviour among young people can to some extent be regarded as a social phenomenon, and that the groups of problem youths can be regarded as a network population characterised by various relations.

Since no random network sample could be obtained, we opted for the best feasible alternative. This involved 'experts' identifying all of the sites frequented by the youngsters. The procedure yielded nine town districts that were particularly affected by nuisance by these youngsters. At the youngsters' favourite hanging-out site in each of these districts, we recruited one problem youth who was willing to act as an interviewer. This led to a total of twelve interviewers (including some reserve candidates). Each of them was expected to interview five of his/her peers, whom they had to recruit themselves. These are referred to as 'respondents' below. Asking the interviewers to recruit more than five respondents each proved infeasible. Each of the respondents was asked to provide information about ten other problem youths they knew, referred to as the 'nominees'. This meant that the interviewers acted as the access points to the network. The selection of interviewers and respondents was based on the criteria listed below.

\section{Selection criteria for interviewers}

The interviewers were selected on the basis of three criteria:

- Geographic criterion. One interviewer was selected for each favourite hanging-out site we had identified. This made allowance for the possibility that the overall network of problem youths consisted of a number of separate networks in the various districts.

- Sociometric criterion. The interviewers were not allowed to know each other. This made allowance for the possibility that the overall network 
consisted of a number of sub-networks that do not know each other, regardless of geographic locations. After the recruitment procedure had been completed, it turned out that only two interviewers knew each other.

- Ethnicity. Each ethnic group was represented by one interviewer. This allowed for the existence of closed, ethnicity-based sub-networks, as it was unclear to what extent problem youths of Moroccan, Turkish and Dutch origin hang out together.

In the end, four interviewers proved unsuitable for the study (one had died, one had been arrested and two failed to do their work properly). These included two interviewers of Moroccan origin. No interviewer of Turkish origin was found. This means that it was impossible to meet the ethnicity criterion, and that we were unable to assess the problems among youths from ethnic minorities.

\section{Selection criterion for respondents}

To ensure that respondents were independent (i.e., did not know each other and did not belong to the same network), the interviewers were instructed to recruit at certain locations within their district, subject to two restrictions: (i) they had to recruit outside their own circle of friends and (ii) they had to recruit at various moments and various favourite hanging-out sites.

\section{The questionnaire}

The questionnaire consisted of two parts. Part 1 included questions on a number of demographic data (age, gender, address) and the behavioural problems of the respondents themselves. Part 2 asked them to name ten other problem youths (the nominees) about whom the respondents were sure that they fitted the description of problem youths (i.e., met criteria a. to e. above). Although a respondent's perceptions of a nominee do not necessarily accurately reflect the nominee's actual problems, we assumed that the frequencies with which the nominees' behavioural problems were reported would provide some indication of the problems prevalent among these youths. Ten nominees per respondent proved to be the maximum that was feasible, as respondents did not know more problem youths. To prevent selection bias, in the sense of respondents nominating only their best friends, they were asked to select three friends (i.e. those with whom they had frequent and close contacts), three acquaintances (with whom they had less frequent and less intimate contacts) and four superficial acquaintances (whom they only knew by name).

This procedure was expected to yield data on 495 problem youths (five respondents each providing ten nominees in each of nine districts). Since the estimated prevalence of externalised problem behaviours among the 17,000 Maastricht inhabitants in the 12-24 year age bracket was $5-10 \%$, this would in 


\section{CHAPTER 5}

the ideal case mean that we would reach about one third to half $(29-58 \%)$ of the target group. The percentage would be lower if there were a considerable number of double nominations or if there were fewer respondents or nominees.

\section{Statistical analysis}

Frank-Snijders estimators (FS estimators) allow the unknown number of problem youths to be estimated from a single-wave snowball sampling. ${ }^{11}$ FS estimators involve drawing a random sample from a network and asking each of the independently selected individuals to name their contacts within the network. Frank and Snijders claim that the estimators they have defined can also be used for non-random samples, provided the samples do not deviate too much from randomness. ${ }^{11}$ We are convinced that the sampling procedure in our Maastricht study met this condition.

The FS estimators were used to estimate the total size $V$ of the population of problem youths, from an initial sample consisting of $n$ respondents. Both estimators use the ratio $q$ of the likelihood that a known respondent is mentioned when respondents name nominees (n-1)/V to the likelihood of an unknown individual being named $(\mathrm{V}-\mathrm{n}) / \mathrm{V}$. The assumption is always that chances are equal for everyone. The estimated values for this ratio,

$q=\frac{(n-1) V}{(V-n) V}=\frac{(n-1)}{(V-n)}$

are then substituted into the equation

$V=\frac{(n-1)}{q}+n$

The first estimator, $\mathrm{V}_{1}$, is based on the number of nominations by respondents. The number of times that respondents name other respondents as nominees is denoted by $r$. The total number of nominations by respondents which relate to nominees not yet known as problem youths (i.e. nominees who are not fellow respondents) is denoted by $s$. (If a nominee is mentioned several times, he is also counted the same number of times.) This estimator is defined as:

$V_{1}=\frac{(n-1) s}{r}+n$ 
The second estimator, $\mathrm{V}_{2}$, is based on the final sample, which consists of the nominees, including respondents mentioned as nominees. We define $t$ as the number of respondents named by other respondents (with a respondent who is mentioned several times being counted only once). The number of newly named problem youths is denoted by $m$ (with nominees who are mentioned several times being counted only once).

$V_{2}=\frac{(n-1) m}{t}+n$

Both of these estimators can be described using 'capture-recapture' terminology, if 'capture' is interpreted as being included in the initial network sample and 'recapture' as being nominated by respondents. It should be noted, however, that, unlike the situation in a capture-recapture procedure, the likelihood of a person being included in the initial network sample depends on the respondents' contacts. In addition, it is likely that the most central youths in the network (the key figures) will be nominated by respondents more often than the less popular ones (the peripheral figures), as the former tend to exhibit more emphatic externalised problem behaviour, attract more attention and are thus more likely to be named by others. Our sample design took this into account (see the selection criteria). Larger numbers of nominations and more double nominations indicate closer links within a network. A more specific discussion of the statistical aspects of the procedure can be found in Frank and Snijders' paper. ${ }^{11}$

\section{RESULTS}

\section{Checking the evaluation criteria}

Eight of the 12 interviewers cooperated fully with the study. They interviewed 37 respondents. The 8 interviewers themselves mentioned 57 peers (nominees), with an overlap of only 3 . This means that the interviewers' circles of friends and acquaintances overlapped very little, indicating that the sub-networks hardly knew each other. Although it later transpired that 2 of the 8 interviewers knew each other, only one nominee was named by both of them. The 8 interviewers and 37 respondents hardly knew each other, as is apparent from the fact that the interviewers themselves were mentioned as nominees only 6 times by the 37 respondents. The 37 respondents mentioned a total of 230 nominees, with 22 nominees being mentioned more than once. The average number of nominees per respondent was 6.2. About three quarters of the nominees $(76 \%)$ were boys, and one quarter (24\%) were girls. The mean age was 19 years (with a 


\section{CHAPTER 5}

standard deviation of 6 years). The mean age of the girls (18 years) was slightly lower than that of the boys. The small number of double nominations allowed us to conclude that the selection criteria for the interviewers and respondents had been sufficiently met.

\section{FS estimators}

Table 1 shows how many of the 37 respondents were assessed to be 'dependent' or 'independent' in terms of time and place (see the discussion of the selection criterion for respondents above). Since interviewers A and B knew each other, they were assessed as dependent. In view of this dependence between interviewers $\mathrm{A}$ and $\mathrm{B}$, two types of basic populations were formed, one based on information derived solely from interviewers $\mathrm{A}, \mathrm{C}, \ldots, \mathrm{H}$ and one based on information derived solely from interviewers $B, C, \ldots, H$. The total number of independent respondents in the first type of basic population was 24: $(A=2)+$ $(\mathrm{C}=2)+(\mathrm{D}=4)+(\mathrm{E}=3)+(\mathrm{F}=4)+(\mathrm{G}=4)+(\mathrm{H}=5)=24$. The second type included 25 respondents judged to be independent.

The individual basic populations were established by adding 3 not previously included respondents for the first type and 2 of such respondents for the second type, while taking care not to add more than one respondent to each interviewer, to avoid dependence. This process included all allowed combinations of respondents assessed to be dependent. Each combination yielded its own estimate. This means that $\mathrm{V}_{1}$ and $\mathrm{V}_{2}$ were estimated 30 times: 18 times for the first basic population $(3 \times 3 \times 2)$ and 12 times for the second basic population $(2 \times 3 \times 2)$. The mean value yielded the following values for the FS estimators:

$\mathrm{V}_{1}=346$, with a standard error of 84

$\mathrm{V}_{2}=301$, with a standard error of 34 .

Table 1 Total numbers of interviews that were dependent and independent in terms of time and place.

\begin{tabular}{|l|l|l|l|}
\hline Interviewer & $\begin{array}{l}\text { Number of inter- } \\
\text { views }\end{array}$ & $\begin{array}{l}\text { Number of dependent } \\
\text { respondents }\end{array}$ & $\begin{array}{l}\text { Number of independent } \\
\text { respondents }\end{array}$ \\
\hline A & 5 & 3 & 2 \\
\hline B & 5 & 2 & 3 \\
\hline C & 5 & 3 & 2 \\
\hline D & 4 & 0 & 4 \\
\hline E & 5 & 2 & 3 \\
\hline F & 4 & 0 & 4 \\
\hline G & 4 & 0 & 4 \\
\hline H & 5 & 0 & 5 \\
\hline Total & 37 & 10 & 27 \\
\hline Interviewers A and B & & \\
\hline
\end{tabular}


Assuming a normal distribution of the point estimates, the true number of problem youths should be within two standard errors from these estimators. This results in a maximum number of problem youths in the town of Maastricht of 514 according to estimator 1 and of 369 according to estimator 2. The minimum estimates are 178 and 233, respectively. Since underestimation is likely when dealing with such hard-to-reach populations, the real minimum number is probably larger. The suspicion that our method underestimated the numbers was confirmed by the fact that we were wholly unable to reach youths from ethnic minorities, and by the fact that the interviewers reported that the respondents were highly suspicious and usually hesitated for some time before participating in the interviews. In addition, incipient problem youths may well not be recognised as such by other problem youths, as the problem is not yet 'clinically manifest'. This phenomenon may also have resulted in central figures being more frequently mentioned in the sample than the more peripheral problem youths, notwithstanding all our attempts to prevent this. This would also result in underestimation by the FS estimators. Assuming that the sampling method we used was an acceptable alternative to a random sample, we therefore conclude that Maastricht has between 300 and 500 problem youths of Dutch origin, most of whom are not known as such to youth care services. This would be about $2-3 \%$ of the town's total population in the $12-24$ year age bracket. This estimate was assessed as realistic by the police, judiciary and various youth care services. Although the range of 300-500 is rather large, the local policymakers mostly wanted to know whether it was a problem of dozens, hundreds or thousands.

We were unable to estimate the number of problem youths of Moroccan origin. It was striking, however, that the 37 respondents of Dutch origin together mentioned only 2 nominees from ethnic minorities. And although the 6 respondents of Moroccan origin had been incorrectly selected, it is interesting to find that they exclusively named nominees of ethnic (i.e., Moroccan) origin. This suggests that the network of Moroccan youths has little or no contact with that of youngsters of native Dutch origin.

\section{DISCUSSION}

The procedure we used in the present study to estimate the number of problem youths has previously been used in several studies of addict populations: heroin users in the Dutch cities of Groningen ${ }^{14}$ and Utrecht ${ }^{17}$ and cocaine users in Barcelona, Rotterdam and Turin. ${ }^{15,18}$ One advantage of this sampling procedure is that the target group members themselves are the ones who do the interviewing, as they have easier access to other target group members than researchers. A disadvantage of the procedure is the risk of selection bias in various phases 


\section{CHAPTER 5}

of the process, viz. when interviewers are being selected, when respondents are being recruited and when respondents have to name nominees. We therefore defined certain criteria to avoid this possible bias, and checked afterwards whether they had been met. Our check suggested minimal selective response in the process of selecting interviewers. As regards the recruitment of respondents, the interviewers did report some reluctance to participate in the study (for fear of being targeted by the police). The fact that we were wholly unable to reach the problem youths of Moroccan origin confirms the risk of selection bias. We tried to prevent selective response as much as possible by ensuring anonymity, using trusted intermediaries to recruit the interviewers and instructing the interviewers carefully. Even so, there may have been some selective response because incipient problem behaviour is not yet 'clinically manifest'. We cannot exclude that applying the FS estimators to problem youths mostly yields the number of manifest cases.

We have only a general impression of whether the network sample we constructed is representative of the total population of problem youths, though experts expressed favourable opinions on its representativeness.

A second issue that needs to be addressed is that of the added value provided by the single-wave snowball sampling procedure and the FS estimators, compared to an estimate by experts based on their 'clinical judgement', especially for the purpose of rough estimates for policymaking purposes. Prior to our study, none of the experts in Maastricht, whether from the police, the judiciary, welfare services, youth care services or schools, were willing to express an opinion on the number of problem youths in the town. When we asked their opinion about our results afterwards, however, they indicated that the findings seemed realistic to them. Even if these experts had been willing to give an estimate before our study, the reliability of such estimates would have remained unclear. The procedure used in the present study would therefore appear to provide a valuable addition to expert opinions, especially for populations to which even experts have trouble getting access.

A major advantage of network sampling is that it yields not only an estimate of the size of the population of problem youths, but also provides some insight into the network structure and contact pattern of the group(s) and the nature of individual problems. This is a type of information that experts often do not possess, whereas it is important in designing preventive measures.

The present study detected 245 individual problem youths. The fact that the lower limit of our estimate is close to the total sample size suggests to us that we have succeeded in 'catching' a large proportion of the problem youth population in the town of Maastricht in our sample. The likelihood of being included in the sample depended on the relations between the respondents. If the respondents we found had all been central figures in their groups, the number of respondents nominated and the number of double nominations should 
have been high, which was not the case. This makes it likely that the network of problem youths in Maastricht consists of a number of unconnected subnetworks, which is actually not an unexpected conclusion. We do realise, however, that we may have missed some sub-networks, which may have led to underestimations.

Our findings allow the conclusion that a single-wave snowball sampling procedure may offer good opportunities for prevalence studies among hard-toreach populations, and that this method provides added value compared to informal estimates by experts based on their fieldwork experience.

A third issue is the reliability of data provided by peers. Since we asked the problem youths themselves to assess whether the nominees exhibited externalised behavioural problems, the question may be raised how reliable such second-hand information is. In a parallel study to the present research, 80 Maastricht problem youths and a control group of the same size took part in indepth interviews with social workers. The findings of these interviews in terms of the prevalence of behavioural problems corresponded remarkably well with those of our single-wave snowball approach. ${ }^{10}$

Although comparisons with findings of similar studies are impeded by differences in the populations studied, our finding that problem youths in Maastricht perceive a great deal of problem behaviour in their social environment indicates that problem behaviour among youngsters should be addressed not only at a personal level, but also at the levels of relationships and situations. This conclusion means that the social environment should be explicitly included in prevention efforts and social work. Just as drug addicts who have gone through detention and rehabilitation will often immediately return to their familiar social network within the drugs scene, problem youths who have been given individual counselling will tend to rejoin their friends and model their own behaviour on them again. As long as the social environment has not changed, or the youngsters have not succeeded in joining a different social network, there is every chance that they will relapse into their old behaviour.

\section{Acknowledgements}

This study was financially supported by the Maastricht municipal authorities. We owe a debt of gratitude to Tom Snijders of Groningen University and Ron Schellings of Maastricht University.

\section{References}

1. Ministerie van Volksgezondheid, Welzijn en Sport. Beleidskader Preventieve en Curatieve Jeugdzorg 1997-2000. Rijswijk, 1996.

2. De Zwart WM, Stam H, Kuipers SBM. Kerngegevens: roken, drinken, drugsgebruik en gokken onder scholieren vanaf 10 jaar. Utrecht: Trimbos-instituut, 1997. 


\section{CHAPTER 5}

3. Van Kampen J. De staat van jeugdig Nederland 1996. Delft: Stichting Jeugdinformatie Nederland, 1996.

4. Landelijke Stichting Buro's Vertrouwensarts inzake Kindermishandeling. Jaarverslag 1995. Utrecht, 1996.

5. Diekstra RFW, Van den Brande JL, Heymans G, Kohnstamm GA, Van Oudenhoven N, Simons PRJ. Jeugd in ontwikkeling: wetenschappelijke inzichten en overheidsbeleid. Den Haag: Wetenschappelijke Raad voor het Regeringsbeleid, 1992.

6. Diekstra RFW, Garnefski N. Scholierenonderzoek 1992. Leiden: Rijksuniversiteit Leiden, 1993.

7. Kousemaker NPJ. Zoeken, vinden, zorgen delen: de ontwikkeling van een paradigma voor onderkenning en pedagogische preventie van psychosociale problematiek in de jeugdgezondheidszorg. Dissertatie. Leiden: Rijksuniversiteit Leiden, 1996.

8. Verhulst FC. Mental health in Dutch children. Rotterdam: Department of Child Psychiatry, Academic Hospital Rotterdam, 1985.

9. De Wijs JPM, Kroesbergen HT, De Bruijn AJM, Gunning-Schepers JL, Ingleby JD. Preventie van psychosociale problematiek in de jeugdgezondheidszorg (II). T Soc Gezondheidsz 1997;75:300-4.

10. Jansen M. Die jeugd van tegenwoordig: een onderzoek naar de omvang en de kenmerken van de Maastrichtse probleemjongeren. Maastricht: GGD Zuidelijk Zuid-Limburg, 1996.

11. Frank O, Snijders TAB. Estimating the size of hidden populations using snowball sampling. J Official Statistics 1994;10:53-67.

12. Meeus W. De psychosociale ontwikkeling van adolescenten. In: Meeus W, 't Hart H (eds). Jongeren in Nederland. Amersfoort: Academische Uitgeverij Amersfoort, 1993.

13. Spreen M. Rare populations, hidden populations, and link-tracing designs: what and why? Bull Methodol Sociol 1992;26:34-58.

14. Intraval 1991. Door regelen in de maat: reacties van drugsgebruikers op maatregelen van overheid en burger. Groningen/Rotterdam: Intraval, 1991.

15. Intraval 1992. in grote lijnen: een onderzoek naar aard en omvang van cocaïnegebruik in Rotterdam. Groningen/Rotterdam: Intraval, 1993.

16. Hendriks VM, Blanken P, Adriaans NFP. Snowball sampling: a pilot study on cocaine use. Rotterdam: Instituut voor Verslavingsonderzoek, 1992.

17. Ten Den C, Bieleman B, De Bie E, Snippe J. Pijn in het hart: onderzoek naar aard en omvang van de harddrugsproblematiek in de stad Utrecht. Groningen/Rotterdam: Intraval, 1995.

18. Bieleman B, Diaz A, Merlo G, Kaplan ChD. Lines across Europe: nature and extent of cocaine use in Barcelona, Rotterdam and Turin, Amsterdam/Lisse: Swets \& Zeitlinger 1993. 


\title{
CASE 1. OVERVIEW OF APPLIED PRACTICAL STRATEGIES
}

\author{
The administrative level
}

The problem was put on the local policy agenda as a result of alarming reports from a local welfare service agency about street corner workers being confronted with an increasing number of problem youths (aged 12-24 yrs). Media reports about youths hanging around in the streets, jeopardizing public safety and civil order, created a favourable political climate [agenda setting +]. A 'window of opportunity' arose and the GGD used the opportunity to start consultations with representatives of the policy, research and practice domains to influence the political agenda. These consultations resulted in a research proposal to study the extent and nature of the problem ${ }^{273}$ which was debated by local politicians and agreed upon ${ }^{275}$ [advocacy +]. The municipal authorities were asked to provide a grant of half of the total investment that was necessary for the application of innovative epidemiological techniques and the incentives for respondents [resource negotiations +]. In anticipation of the reply of the municipal authorities, the practice and research work cycles were already started up, prompted by the sense of urgency among the practitioners. This meant that the GGD was taking certain financial risks, though these were deemed acceptable. The formal administrative decision to provide the grant came ten months later, just two months before the official presentation of the final research findings ${ }^{276}$.

No formal agreements were made between local authorities and institutional directors, except for a letter of confirmation of the task assignment from the municipal authorities related to the grant. The proposal which was accepted by local politics had to be jointly carried out by a local welfare service agency (Stichting Trajekt) and an addiction care service (Mondriaan Zorggroep), with the support of the Department of Psychiatry and Neuropsychology of Maastricht University. Support by the university department was not asked for until the research preparations started. The collaboration in this case cannot be regarded as a coalition, as by modern standards, a coalition is a more formal working partnership ${ }^{233}$. This was definitely not the case, as local authorities, the GGD Board and the professors or social entrepreneurs from the university never met to discuss either problem youths or collaboration [coalition building -]. Nor did the partners participate in any lobbying efforts to achieve collaboration between the policy, practice and research domains in tackling the problem [sidestepping the formal system - ]. There was one person who lobbied for collaboration, viz. the project leader, but she did not have the formal status at the administrative level to achieve it. 


\section{CHAPTER 5}

\section{The institutional level}

The actors that had to play a role in the process to facilitate collaboration were the managing director of the GGD, the heads of the welfare and addiction care services, a professor from the Department of Psychiatry and Neuropsychology of Maastricht University, and the head of the Department of Education, Culture, Welfare and Health of the Maastricht municipal administration. None of them explicitly articulated the need for organizational development to facilitate collaboration. None of the managers initiated activities to diagnose the collaboration process and goals [diagnosis-].

An action plan was drawn up, including only activities related to the project content, and the activities were restricted to the domains of practice and research. None of the managers paid any attention to the collaboration process as such. The policy domain was not involved, and no strategies were applied to get them involved [activities and interventions -]. The head of the municipal administration department (policy domain) never assigned civil servants to participate in the meetings to prepare the action plan. She only coordinated the financial aspects, i.e. the grant. The action plan did not identify the key players whose commitment was needed, nor did it anticipate a commitment strategy [commitment -]. The collaboration had a rather informal character, without a management structure of its own. GGD, the welfare agency and the addiction care service facilitated the professionals involved, in terms of both time and competence. Later, Maastricht University became involved, as a result of personal interest in the content [intersectoral management structure -].

For administrative reasons, formal agreements were made for the interim and final results to be communicated to municipal civil servants and the relevant alderman. Interim feedback was embedded in the existing structure of youth policy consultations (Beleidsoverleg Jeugd, BOJ), chaired by the alderman, in the presence of civil servants responsible for youth policy and public health policy, and involved public and private agencies. The BOJ met every two months. The final findings and recommendations were presented to the mayor and aldermen in the presence of the heads of the GGD, welfare agency and addiction care service, followed by a panel discussion with experts on education, compulsory education inspection, youth crime prevention, indecency offences, policing and youth health care. In retrospect, this communication structure was a one-way communication process, and mainly served the unilateral goals of the municipal administration to monitor the outcome of financial investments [communication structure +- ].

The evaluation design was assessed, though the assessment was restricted to content aspects. None of the institutional managers involved felt there was a need to evaluate the collaboration process as such [evaluation -]. 
Resources were available (or promised) thanks to a grant from the Department of Education, Culture, Welfare and Health of the Maastricht municipal administration. The institutional managers assigned staff to implement the activities. However, no budgets were earmarked for capacity building to disseminate the innovative epidemiological techniques used in the research to practitioners and to get them to adopt these techniques in everyday practice [resources +- ].

Practical strategies to formulate a mission statement on collaboration were not considered beforehand, nor was a PR policy to stress the innovative nature of the intersectoral collaboration [mission statement -], [interviews with role models -]. The issue was considered to belong to the regular tasks of the agencies involved. The representatives of the research and practice domains implicitly assumed that policy makers would be prepared to collaborate on the issue, and to subsequently apply the results, which is why no practical strategies were applied to achieve full transparency about the policy goals. Institutional managers approved the exchange of internships between the practice and research partners. These internships were organized to allow those involved to experience the problem first-hand, to sensitize working cultures and to implement research in the practice domain. The university's Department of Social Medicine agreed to organize an internship for the field research and the analysis. The internships were focused on research in a real-life setting to fill the knowledge gaps. The policy domain was not involved in the internships [internships +-]. Rendering services between domains and access to the tools of the other domains were achieved more or less automatically, but were restricted to the practice and research domains [rendering services, access to tools +- ]. A formal consultation structure was linked to the communication at the regular BOJ meetings. In addition, various consultations were organized between representatives of the practice and research domains [formal and informal consultations $+]$. The institutional management did not prepare practical strategies for capacity building [diagnosis and feedback -], [recruiting and retaining staff -], [performance indicators and professional development -].

No kick-off conference was organized by the institutional managers [kick off conference - ]. The project leader frequently contacted all partners involved and communicated about the progress of the research in the practice setting and about the policy recommendations. In retrospect, however, she lacked the hierarchical power or internal management support that would have allowed her to influence the organizational development at institutional management level among the other partners [sidestepping the formal system +- ]. 


\section{CHAPTER 5}

\section{The individual level}

At the individual level, various practical strategies were applied. An intersectoral working group was formed, chaired by the GGD, which met every six weeks, and included researchers and practitioners. The meetings were chaired by the project leader, who identified the initial situation, the progress and the collaboration process, and gave regular feedback to the individual professionals and, when necessary, to the managers [diagnosis and feedback +]. The practical strategies most frequently initiated by the project leader were group discussions, learning by doing and feedback by peer networking. The practitioners, who came from various fields, like addiction care, welfare, youth health care, street corner work, the police and the school health promotion service, formed a multidisciplinary group, together with researchers from the departments of Psychiatry and Neuropsychology, Social Medicine and Medical Statistics of Maastricht University. The expert of the Department of Medical Statistics was familiar with the statistical method of snowball sampling technique. There was enough room for discussion, and peers, for instance from the field of addiction, exchanged operational methods with practitioners from other fields [group discussion in multidisciplinary groups + ], [feedback by peer networking + ]. Learning by doing was stimulated by detailed written action plans, formats, instructions and other materials to facilitate individual performances [learning by doing +], [detailed action plan, formats, materials +]. No practical strategies were applied to organize lectures to explore future implications of the results or to continue the collaboration beyond the project period [lectures and discussion -]. The idea of organizing master classes to learn specific skills was not considered [master classes with skills training -]. In retrospect, a master class on the snowball technique and statistical analysis would have furthered the epidemiological expertise available at the GGD, allowing them to estimate the magnitude and problems of various 'hidden populations', such as asylum seekers, homeless people, problem youths, victims of sexual and other types of intimidation, etc.

Practitioners and researchers coached each other on publications and presentations. They published in a peer-reviewed Dutch journal and various professional journals within the research and practice domains. The project leader and the main researcher gave presentations at conferences, making knowledge accessible to a wider Dutch audience277,278. No practical strategies were applied to involve the policy domain in publishing efforts, because the civil servants were not closely involved in the actual running of the project. Stimulating researchers and practitioners to publish in policy journals proved unfeasible, due to the absence of representatives of the policy domain [inservice coaching to publish beyond one's own niche +-$]$. 


\section{Comments}

This case illustrates the weakness of an ad-hoc collaboration between the policy, practice and research domains. The collaboration was based on the content of a problem, on which individual professionals worked together intensively and enthusiastically, but for which no explicit links between institutions were created. Nor was there sufficient transparency about the goals expressed at the administrative level. In this case the problem was perceived as socially, practically and scientifically relevant, and the local authorities agreed to initiate further investigation, carried out under the responsibility of the GGD. The GGD then initiated the involvement of the research domain. Researchers and practitioners were able to report evidence-based results on problem youths. They jointly went through the first four steps of the work cycle (see chapter 2). Researchers and practitioners assumed that the evidence-based results would enter the policy work cycle at step 1 in order to strengthen local youth policy. As we concluded in chapter 2, steps 4 and 1 of the various work cycles should be closely linked, in order to improve public health incrementally by consecutive concerted actions. Step 1 requires the agreement of stakeholders at the administrative level to put an issue on the local policy agenda, to lobby for and advocate its relevance and to start resource negotiations. At this point, the administrative level had to initiate a new run of the work cycle. However, stakeholders at this level were not able to maximize power, despite media attention ${ }^{279}$. As institutional managers and social entrepreneurs had not achieved any linkage during the first cycle, they were not prepared to become actively involved in practical strategies like advocacy, lobbying, resource negotiations etc., which were necessary for the second run of the work cycle. The thirteen differences between the three work cycles were not given conscious attention and consideration. The municipal administration appeared to have interpreted the task they assigned to the GGD as not involving any further obligations on their part as regards the development of youth health policy. The collaboration evaporated as soon as the first run of the work cycle had been completed. Responsibilities after the project were unclear. The final goals and interconnections between the policy, practice and research domains had not been transparently defined beforehand. Gradually, the subject of problem youths disappeared from the policy agenda.

At institutional level, collaboration continued, but only on a contentoriented and ad hoc basis, as had already been common practice, and with less attention being devoted to problem youths, due to insufficient funding for further practice and research. Since practical strategies on working climate, working culture and capacity were hardly implemented, no progress was made in organizational development. Interventions from the professionals to change the inertia at the institutional and administrative levels did not materialize. 


\section{CHAPTER 5}

The collaboration between the practice and research domains contributed to evidence-based public health results, as was illustrated by the valid prevalence estimations of a hidden population, and the extent of their problems. However, the research instruments used, i.e. the snowball sampling technique and the statistical analysis, were not adopted into the GGD's routine practice, and youth health policy adjustments were very poorly. In this case research did neither lead to evidence-based practice nor evidence-based policy. We can conclude that ad-hoc collaborations between the policy, practice and research domains will not achieve their intended purpose when the administrative goals are unclear and the links at the institutional level are lacking, even when practitioners and researchers are able to collaborate with great enthusiasm. 
MULTIPLE CASE STUDY

\subsection{CASE 2. INTRODUCTION}

Sexually transmitted diseases in the Dutch province of Limburg in 1997: prevalence figures based on a survey among general practitioners and specialists and on data from microbiology laboratories

The ambition in this collaborative project was to improve the quality of the regional surveillance system for sexually transmitted diseases (STDs) by initiating a collaborative network of medical specialists from the Maastricht university hospital, the regional microbiology laboratories, the general practitioners and the GGD, in order to obtain valid data to strengthen STD policy in the province of Limburg.

Sexually transmitted diseases are regarded as a public health problem because of their high incidence and prevalence, the large number of asymptomatic infections, the chronic or persistent course of infections, their role in the development of specific forms of cancer, and the development of antimicrobial resistance, jeopardizing adequate treatment ${ }^{280}$. In the Netherlands, notification of some STDs (e.g., gonorrhoea, syphilis and acute hepatitis B) has been mandatory since 1976, while for others (e.g., Chlamydia trachomatis infections, condylomata acuminata, genital herpes) it is not. For those diseases for which notification is not mandatory, no accurate data are available locally. Developing a regional preventive policy requires systematic surveillance to obtain epidemiological data on incidence and prevalence. The prevailing national surveillance system, however, had too many shortcomings to support regional policy. A major shortcoming was the representativeness of the STD clinic populations that served as the surveillance population, as most of them lived in the western part of the Netherlands ${ }^{281}$. Since 1985, the GGD for Southern Limburg and the Maastricht university hospital have operated an STD clinic to address the problem of STDs and to improve the surveillance system and subsequently the local STD policy. The article included below illustrates the need for surveillance improvements. 


\section{CHAPTER 5}

Published (in Dutch) in Nederlands Tijdschrift voor Geneeskunde 2000;144:608-12

Sexually transmitted diseases in the Dutch province of Limburg in 1997: prevalence figures based on a survey among general practitioners and specialists and on data from microbiology laboratories

C.J.M. Henquet, MD, Department of Dermatology, University Hospital Maastricht

M.W.J. Jansen, MSc, GGD Zuid Limburg

P.J.S. Buwalda, MD, Department of Dermatology, Faculty of Medicine, Maastricht University

H.A.M. Neumann, Professor at the Department of Dermatology, University Hospital Maastricht 


\begin{abstract}
Objective. To determine the incidence of sexually transmitted diseases (STDs) and to compare data reported by general practitioners (GPs) and specialists with those reported by microbiological laboratories.

Design. Retrospective survey.

Method. All 593 GPs and gynaecologists, dermatologists and urologists in the Dutch province of Limburg were asked in 1998 to complete a questionnaire about the numbers of cases of Chlamydia trachomatis, condylomata acuminata, genital herpes and gonorrhoea in 1997, classified by sex, age and diagnostic test result. Data were compared with information gathered from the six laboratories of clinical microbiology. Results for gonorrhoea were compared with those of a 1985 study.

Results. The survey response rate was $75 \%$. A total of 2730 cases were reported (32 per 10,000 of the population). Infection with C. trachomatis was the most frequent STD (46\%), followed by condylomata acuminata $(28 \%)$, genital herpes $(17 \%)$ and gonorrhoea $(8 \%)$. Eighty-four percent of the diseases occurred in persons younger than 35 years of age, and $66 \%$ occurred in women. The GPs saw $79 \%$ of the STDs. They diagnosed $25 \%$ of the cases of 'gonorrhoea' solely on the basis of the clinical picture. Compared to 1985, the number of gonorrhoea cases in Limburg had dropped dramatically in 1997. Of those who answered the questions about warning partners of persons with STDs (about 50\% of the respondents), $87 \%$ reported that they had warned their partners. The laboratory data indicate that the incidence of $\mathrm{C}$. trachomatis infection was 3.2 and that of gonorrhoea 0.6 per 10,000 inhabitants of the province. According to the GPs and specialists, $50 \%$ of the cases of $\mathrm{C}$. trachomatis had been diagnosed on the basis of cultures, whereas the laboratories reported using cultures in only $2 \%$, ligase chain reaction in $20 \%$ and polymerase chain reaction in $78 \%$ of cases.
\end{abstract}

\title{
INTRODUCTION
}

Notwithstanding our greatly increased knowledge about the prevention of HIV infection and sexually transmitted diseases (STDs) in general, such diseases still cause considerable health problems in the Netherlands. Although recent decades have seen changes in sexual behaviour, there is still a considerable gap between knowledge and behaviour. ${ }^{1}$ Various registration systems show that there has been a shift from the 'classic' venereal diseases gonorrhoea and syphilis to 'new' STDs like Chlamydia trachomatis infections and condylomata acuminata. ${ }^{2-4}$ Reported cases of these infections have increased in recent years, partly as a result of improved diagnostics. Data on the prevalence of STDs in the Netherlands are only available from healthcare registration systems and 


\section{CHAPTER 5}

regional epidemiological databases.5,6 As a result of geographic bias (large cities in the west of the country being overrepresented) these data cannot simply be extrapolated to the general Dutch population or - in our case - that of the south-eastern province of Limburg. It is unknown to what extent the Limburg prevalence figures differ from those in the west of the country.

To assess the prevalence of STDs in Limburg, a written questionnaire survey was organised among healthcare workers in 1998, inquiring about the incidence of the three most common STDs, C. trachomatis infection, condylomata acuminata and herpes genitalis. The present study design, relating to the year 1997, was identical to that of a 1985 study into the prevalence of gonorrhoea in Limburg and also included gonorrhoea, in order to allow comparisons between the two assessments. ${ }^{6}$

This article discusses the 1997 prevalence of these four STDs in the province of Limburg, as well as the difference in the prevalence of gonorrhoea between 1985 and 1997 and the differences between the numbers reported by healthcare workers and by the clinical microbiology laboratories. Finally, it comments on the methods used and suggests improvements to the existing STD surveillance system, to prevention programmes in primary health care and to the supplementary diagnostic training offered to healthcare workers.

\section{METHODS}

In the summer of 1998, all 593 general practitioners (GPs), gynaecologists, dermatologists and urologists in the province of Limburg were sent a questionnaire on the prevalence of C. trachomatis infection, condylomata acuminata, herpes genitalis and gonorrhoea in 1997. They were asked to provide an estimate from memory, since few were using computerised patient files at that time. If the doctors had not responded after two weeks, the questionnaire was administered by telephone. Questions included in the survey concerned the numbers of cases of these four STDs, age and gender of the patients (and risk group in the case of gonorrhoea), the diagnostic workup used for gonorrhoea and C. trachomatis infection, and efforts undertaken to trace contacts. The GPs were explicitly asked to include only those cases they had diagnosed and treated themselves, to minimise the overlap between GPs and specialists.

The six laboratories of clinical microbiology in the province were asked to report the numbers of tests for C. trachomatis infection and gonorrhoea requested in 1997, and their results. They were asked to specify the diagnostic tests used for C. trachomatis infection (culture, enzyme-linked immunosorbent assay (ELISA), ligase chain reaction (LCR), polymerase chain reaction (PCR), serology, gene probes or other). 
The national Health Care Inspectorate provided data on the number of cases of gonorrhoea (which is a notifiable disease in the Netherlands) reported in Limburg in 1997. Gonorrhoea prevalence data for 1985 were derived from an earlier study. ${ }^{6}$

\section{RESULTS}

The response rate to the questionnaire survey was $75 \%(442 / 593)$. Response rates among the specialists were highest for the dermatologists $(24 / 30,80 \%)$, followed by the gynaecologists $(29 / 43,67 \%)$ and the urologists $(15 / 25,60 \%)$. Response among GPs was 76\% (374/495). Reasons given for non-response included illness $(4 \%)$, having terminated the practice $(12 \%)$, refusal and lack of data $(84 \%)$. All six clinical microbiology laboratories in Limburg provided data.

\section{Data from GPs and specialists}

The total number of cases of the four STDs reported by GPs and specialists was 2730 . C. trachomatis infection was the most commonly reported disease, with 1255 cases $(46 \%)$, followed by condylomata acuminata with 776 cases (28\%), herpes genitalis with 474 cases $(17 \%)$ and gonorrhoea with 225 cases $(8 \%)$. Of all STD cases, 1805 (66\%) occurred in women and 898 in men (while the patient's gender could not be ascertained in 27 cases). Among female patients, the most common STD was C. trachomatis infection (977 of the 1805 cases; 54\%), while the most common STD among men was condylomata acuminata $(329 / 898 ; 37 \%)$. The male-female ratios for the various STDs were $20 \%$ versus $80 \%$ for $\mathrm{C}$. trachomatis infection, $42 \%$ versus $58 \%$ for condylomata acuminata, $28 \%$ versus $72 \%$ for herpes genitalis and $85 \%$ versus $15 \%$ for gonorrhoea.

Thirty-seven percent of the cases of these four STDs (1013/2730) were diagnosed in patients younger than 25 years and 84\% (2283/2730) in patients younger than 35 . Condylomata acuminata and herpes genitalis were relatively common among persons older than 35 . Based on the $75 \%$ response rate and assuming this was non-selective, these data allowed us to calculate the 1997 incidence of these four STDs in Limburg (total population 1,136,199) as 32 per 10,000 inhabitants $(4 / 3 \times 2370 / 1,136,199)$. The incidence of C. trachomatis infection was 14.7 , that of condylomata acuminata 9.1 , that of herpes genitalis 5.6 and that of gonorrhoea 2.6 per 10,000 inhabitants.

The GPs diagnosed 79\% (2157) of the cases of the four STDs; 78\% (977) of the cases of C. trachomatis infection, 74\% (571) of the cases of condylomata acuminata, $85 \%$ (398) of the cases of herpes genitalis and 92\% (207) of the cases of gonorrhoea. The corresponding 1985 figure for gonorrhoea was $78 \%$. Thirty percent of the cases of gonorrhoea in 1997 (67/225) occurred in the risk 


\section{CHAPTER 5}

group of prostitutes or their clients, almost twice the percentage in 1985 (380/2421). In 1997, the GPs diagnosed $25 \%$ of the gonorrhoea cases $(51 / 207)$ purely on the basis of the clinical picture, compared to $18 \%(337 / 1884)$ in 1985. Forty-four percent of the healthcare workers (194) did not answer the question about warning partners of infected persons, with a maximum of $59 \%$ for gonorrhoea (261). Of the care workers who did answer this question, 87\% (268) indicated they would warn the patient's partner (or have him/her warned) in the case of C. trachomatis infection, while $90 \%$ (163) would do so in the case of gonorrhoea.

\section{Laboratory data}

Of the six laboratories that provided us with data on the numbers of test requests and the test results, four gave further details on the diagnostic tests they had used. Excluding serological tests, 363 (5.3\%) of the 6796 tests for C. trachomatis were positive. Of the tests for which specific information was available on the technique used, 284 of the positive test results $(78.2 \%)$ were based on LCR or PCR tests, $77(21.2 \%)$ on gene probe tests and $2(0.6 \%)$ on culture tests. Positive serological test results $(459 / 1517 ; 30 \%)$ could not be specified in terms of genital or pulmonary C. trachomatis infections, nor whether IgM or IgG antibodies were involved. There were also considerable differences between the laboratories in the percentage of positive serological test results (ranging from 0 to $78 \%$ ). In view of this spread and the fact that we regard serological tests as unsuitable for the diagnosis of current genital C. trachomatis infections, these serological test results were disregarded. Of the tests that led to positive results for C. trachomatis infection, $192(53 \%)$ had been requested by the GPs, $127(35 \%)$ by the gynaecologists, $29(8 \%)$ by the dermatologists, 4 $(1 \%)$ by the urologists and $11(3 \%)$ by others. The healthcare workers reported that $50 \%$ of the cases of $\mathrm{C}$. trachomatis had been diagnosed with the help of cultures, whereas the laboratories reported this in only $2 \%$ of the cases. The healthcare workers reported that $20 \%$ of the cases of C. trachomatis had been diagnosed with the help of LCR or PCR tests, whereas the laboratories reported this in $78 \%$ of the cases.

Of the 8054 cultures for Neisseria gonorrhoeae, $66(0.8 \%)$ were positive, compared to $5.3 \%$ in 1985 . The percentage of cases of gonorrhoea reported by the healthcare workers that were confirmed by a positive culture increased from $15(361 / 2421)$ in 1985 to $29(66 / 225)$ in 1997.

The data from the six laboratories show that the incidence of C. trachomatis infection in 1997 was $3.2(363 / 1,136,199)$ and that of gonorrhoea 0.6 $(66 / 1,136,199)$ per 10,000 inhabitants of the province of Limburg. 


\section{Data from the Health Care Inspectorate}

In 1997, 51 cases of gonorrhoea were reported to the national Health Care Inspectorate, that is, $23 \%$ of the number of cases reported by the healthcare workers in our survey, and $77 \%$ of the number reported to us by the six laboratories.

\section{DISCUSSION}

The order of frequency for the various STDs in the Dutch province of Limburg found in the present study corresponds to the 'STD top ten' published by STI AIDS Netherlands, the national institute for Sexual Transmitted Infections and AIDS control in the Netherlands. The huge drop in the number of gonorrhoea cases between 1985 and 1997 is in agreement with the national trend. ${ }^{2}$ This also explains the decreasing percentage of positive gonorrhoea cultures.

In agreement with national trends, most STDs occurred among those aged below 35 years $(84 \%$ in Limburg versus $75 \%$ nationally, based on data from the regional public health services). ${ }^{4}$ The most common STD among women was C. trachomatis infection, while that among men was condylomata acuminata. The male-female ratios we found for C. trachomatis infection (20\% versus $80 \%$ ) and for gonorrhoea (85\% versus 15\%) differed from those reported in a national study by the regional public health services (which reported ratios of $50 \%$ vs. $50 \%$ and $75 \%$ vs. $25 \%$, respectively). ${ }^{4}$ This may have been caused by differences in the populations studied: the population in our study largely corresponds to the population of general practice patients, with more consultations by women than by men. In view of the greater awareness among health care workers of C. trachomatis infection in women, this may have led to overreporting. Conversely, gonorrhoea may have been overreported among men, as the diagnosis of urethritis is often made by GPs without further diagnostic workup and is then recorded as gonorrhoea on the basis of the history.

In our study, $79 \%$ of patients with a potential STD who consulted a doctor were seen by their GP. There was a considerable discrepancy between the healthcare workers and the laboratories as regards the type of diagnostics they reported to have been used for $\mathrm{C}$. trachomatis infections. This was probably due to lack of knowledge among the healthcare workers about current diagnostic techniques. Samples taken from patients were often described as 'cultures', and GPs in particular were often unaware of amplification techniques like LCR and PCR. These amplification techniques are often preferred since they do not impose special requirements on the samples and the tests are valid and reliable. ${ }^{7-11}$ Knowledge of modern diagnostic techniques should be a routine component of the medical school curriculum and of supplementary and refresher 


\section{CHAPTER 5}

courses for doctors working in this field. Dermato-venereological centres working in the field of STDs could play an important role in this respect.

Primary healthcare has an important responsibility for STD prevention, particularly that of $\mathrm{C}$. trachomatis infection. Consistent condom use is an essential preventive measure. ${ }^{12-14}$ We feel that consultations for oral contraceptives offer a good opportunity to point out the importance of safe sex to young people, putting special emphasis on skills and risk perceptions. ${ }^{15,16}$ In view of their specific expertise in this area, the regional public health services can provide valuable support.

The high degree of non-response to our question about warning partners of infected persons suggests that this is not consistently being done. Since C. trachomatis infection is the main cause of secondary infertility among women, early detection, including consistently warning partners, is crucial. ${ }^{17-19}$ Hence, healthcare workers should be more consistent as regards identifying the contacts of infected persons. It is useful to offer preventive, behaviour-oriented interventions when patients consult healthcare workers about STDs. ${ }^{20}$ We therefore recommend close collaboration between GPs, dermatovenereological centres and regional public health services, allowing integrated provision of medical-diagnostic, curative and preventive interventions.

The discrepancies between healthcare workers, laboratories and the Health Care Inspectorate as regards the numbers of STDs raise some questions about the reliability and validity of the method we used. Various explanations could be offered for the fact that the healthcare workers reported over three times as many cases of gonorrhoea and C. trachomatis infection as the number of positive test results reported by the laboratories. One option is that doctors often offer treatment without further diagnostics, for fear of missing any possible STDs. In addition, healthcare workers often initiate treatment on clinical suspicion of an STD alone, and may afterwards remember the case as 'positive', even if the laboratory test result turns out to be negative. ${ }^{5}$ Also, considerations of social desirability may have induced healthcare workers to overestimate in their responses.

Since the written questionnaire did not produce sufficiently accurate data on the true number of cases, the validity of this method (i.e., the level of systematic error) should be further examined. The incidence rates derived from the laboratory records (3.2 per 10,000 for C. trachomatis infection and 0.6 for gonorrhoea) and those derived from the questionnaire survey (14.7 and 2.6, respectively) differ considerably from the national incidence estimates of 300800 per 10,000 inhabitants aged 15-40 years for C. trachomatis infection and 0.9 per 10,000 for gonorrhoea. ${ }^{6}$ Nor do regional outpatient data on STD examinations provide useful comparisons, in view of the selective patient population. Since there is no national system of STD surveillance in the Netherlands, it is very difficult to detect and interpret trends. 
A systematic STD surveillance system would be of great value for national, regional and local policy development. Although the method reported in the present article may fill this gap to some extent, we feel that a more valid and reliable instrument ought to be developed. ${ }^{6}$ This should be done in close collaboration with primary healthcare workers, in view of their crucial role in the system.

\section{Aknowledgement}

We thank dr. J.S.A. Fennema, epidemiologist of the Amsterdam Public Health Service, who commented on the manuscript.

\section{References}

1. Evans BA, McCormack SM, Bond RA, MacRae KD. Trends in sexual behaviour and HIV testing among women presenting at a genitourinary medicine clinic during the advent of AIDS. Genitourin Med 1991;67:194-8.

2. Van de Laar MJW. On the epidemiology of sexually transmitted diseases in the Netherlands. Dissertation. Amsterdam: Universiteit van Amsterdam, 1997.

3. Van Duijnhoven YTHP. Epidemiology and control of sexually transmitted diseases in a Rotterdam STD clinic. Dissertation. Amsterdam: Universiteit van Amsterdam, 1998.

4. Van de Laar MJW, Termorshuizen F. Registratie van seksueel overdraagbare aandoeningen bij GGD-en. Jaarverslag 1996. Rapportnr. 441500.007. Bilthoven: Rijksinstituut voor Volksgezondheid en Milieu, 1998.

5. Kessels AGH, Bisscheroux PFLA. Gonorroe in Limburg. Verslag van een telefonische enquête. Geleen: Gezondheidsdienst Westelijke Mijnstreek, 1986.

6. Van de Laar MJW, Termorshuizen F. Seksueel overdraagbare aandoeningen (SOA). In: Maas IAM, Gijsen R, Lobbezoo IE, Poos MJJC (eds). Volksgezondheid Toekomst Verkenning 1997. Deel 1. De gezondheidstoestand: een actualisering. Bilthoven: Rijksinstituut voor Volksgezondheid en Milieu, 1997.

7. De Barbeyrac B, Rodriguez P, Dutilh B, Le Roux P, Bébéar C. Detection of Chlamydia trachomatis by ligase chain reaction compared with polymerase chain reaction and cell culture in urogenital specimens. Genitourin Med 1995;71:382-6.

8. Grun L, Sheldon J. Does ligase chain reaction assay of urine in the diagnosis of Chlamydia trachomatis offer significant improvement over existing diagnostic tests? - a critical appraisal of the evidence. Genitourin Med 1996;72:435-9.

9. Stary A. Chlamydia screening: which sample for which technique? Genitourin Med 1997;73:99-102.

10. Quinn TC, Welsh L, Lentz A, Crotchfelt K, Zenilman J, Newhall J, et al. Diagnosis by Amplicor PCR of Chlamydia trachomatis infection in urine samples from women and men attending sexually transmitted disease clinics. J Clin Microbiol 1996;34:1401-6.

11. Bassiri M, Hu HY, Domeika MA, Burczak J, Svensson LO, Lee HH, et al. Detection of Chlamydia trachomatis in urine specimens from women by ligase chain reaction. J Clin $\mathrm{Mi}-$ crobiol 1995;33:898-900.

12. Ford K, Wirawan DN, Fajans P, Thorpe L. Aids knowledge, risk behaviors, and factors related to condom use among male commercial sex workers and male tourist clients in Bali, Indonesia. AIDS 1995;9:751-9.

13. O'Connor CC, Berry G, Rohrsheim R, Donovan B. Sexual health and use of condoms among local and international sex workers in Sydney. Genitourin Med 1996;72:47-51. 


\section{CHAPTER 5}

14. Tanaka M, Nakayama H, Sakumoto M, Matsumoto T, Akazawa K, Kumazawa J. Trends in sexually transmitted diseases and condom use patterns among commercial sex workers in Fukuoka City, Japan,1990-93. Genitourin Med 1996;72:358-61.

15. Darrow WW. Health education and promotion for STD prevention: lessons for the next millennium. Genitourin Med 1997;73:88-94.

16. Hale PJ, Trumbetta SL. Women's self-efficacy and sexually transmitted disease preventive behaviors. Res Nurs Health 1996;19:101-10.

17. Weström L, Wölner-Hanssen P. Pathogenesis of pelvic inflammatory disease. Genitourin Med 1993;69:9-17.

18. Weström L, Joesoef R, Reynolds G, Hagdu A, Thompson SE. Pelvic inflammatory disease and fertility. A cohort study of 1,844 women with laparoscopically verified disease and 657 control women with normal laparoscopic results. Sex Transm Dis 1992;19:185-92.

19. Ankum WM, Mol BW, Veen van der F, Bossuyt PM. Risk factors for ectopic pregnancy: a meta-analysis. Fertil Steril 1996;65:1093-9.

20. Gunn RA, Rolfs RT, Greenspan JR, Seidman RL, Wasserheit JN. The changing paradigm of sexually transmitted disease control in the era of managed health care. JAMA 1998;279:680-4. 


\section{CASE 2. OVERVIEW OF APPLIED PRACTICAL STRATEGIES}

\section{The administrative level}

As was mentioned in chapter 2, the stakeholders at the administrative level are the local authorities, the GGD Board and a professor or director from the local university, as a social entrepreneur. In this case, the local authorities were responsible for STD prevention, and for coordinating prevention with curative medicine on the basis of epidemiological data, which the local authorities are obliged to collect as part of their regular WCPV tasks. Since quality improvement on these issues was perceived as an institutional rather than an administrative concern, the role of the local authorities in quality improvement was limited. In addition, getting the independent group of general practitioners (GPs) involved in the collaboration, and then motivating them to meet higher surveillance standards, required at least some commitment from this group, and probably required enforcement by national guidelines. It was unlikely that local policy makers would have much impact. Commitment by the regional association of general practitioners and the regional organization of microbiology laboratories seemed necessary, as they both have a rather influential role in the formulation of new policy affecting their colleagues. For this reason, practical strategies had to be applied to involve these stakeholders as representatives of surveillance policy makers, instead of the local authorities.

However, no practical strategies were applied at this administrative level [sidestepping the formal system -], [advocacy -], [coalition building -], [agenda setting -], [resource negotiations -]. Actually, a collaboration between the regional association of general practitioners, the regional organization of microbiology laboratories, the board of the Maastricht university hospital and the board of the GGD was not anticipated.

\section{The institutional level}

Management support was provided by two of the four partners which formally agreed to participate in the project. The regional microbiology laboratories and the regional association of GPs expressed their willingness to supply the requested information, but time constraints prevented them from participating in the project. The project leader drew up a problem diagnosis, which was restricted to the content of the project. No diagnosis was provided about the collaboration process as such [diagnosis -]. An action plan was formulated and approved by the management, but it did not include interventions or activities related to the collaboration process [activities and interventions -]. A commitment strategy was not consciously considered by any of the institutional man- 


\section{CHAPTER 5}

agers [commitment -]. A separate management structure was not deemed necessary, as the main partners, i.e. the Maastricht university hospital and the GGD, were already collaborating in the STD clinic, which was headed by a professor of the Department of Dermatology. However, the regional microbiology laboratories and the regional association of GPs were not represented [intersectoral management structure -]. The communication structure corresponded to the organizational structure of the STD clinic, and no separate practical strategies were used [communication structure -]. The collaboration process was not evaluated as such [evaluation -]. No practical strategies were applied to allocate funds for the scarce resources, staff were not assigned to specific tasks, capacity requirements were not surveyed nor financially earmarked [resources -].

No practical strategies were added to contribute to the working climate [mission statement -], [interviews with role models -]. The project management agreed to fund an internship at the university's Faculty of Medicine for the field research and analysis. This decision contributed to a linkage between the working cultures of the practice and research domains, without the policy domain being involved [internships +-]. Rendering services between domains, access to the tools of the other domains, and formal and informal consultation structures were all common practice within the STD clinic, although there were no policy links with GPs and microbiology laboratories. No action was undertaken to change this situation [rendering services, access to tools +- ], [formal and informal consultations+-]. No activities were initiated regarding capacity building [diagnosis and feedback -], [recruiting and retaining staff -], [performance indicators and professional development -], [kick-off conference -].There was no strong leadership with persuasive power to convince the management of the necessity of collaboration and the need for organizational development [sidestepping the formal system -].

\section{The individual level}

The project team was a small team of only five persons, with limited time to spend on the issue, which did not provide an initial diagnosis with feedback to the management to assess the available and required expertise [diagnosis and feedback -]. Practitioners and researchers, who were keen to work on the problem as such, initiated practical strategies that were easy to organize and appeared useful, such as group discussions with interpersonal feedback, learning by doing and a detailed action plan [group discussion in multidisciplinary groups + ], [learning by doing + ], [detailed action plan, formats, materials + ]. No further strategies were anticipated [feedback by peer networking -], [lectures and discussion -], [master classes with skills training -]. 
Mutual in-service coaching was applied as a strategy to stimulate publications beyond people's own niche. This resulted in a publication in a Dutch peer-reviewed journal and one in a not peer-reviewed specialist journal, to disseminate the knowledge among a Dutch audience ${ }^{282,283}$. Policy makers did not participate in the mutual coaching between practitioners and researchers [inservice coaching to publish beyond one's own niche +-$]$.

\section{Comments}

The main goal of initiating collaboration between medical specialists of the Maastricht university hospital, the regional microbiology laboratories, the general practitioners and the GGD practitioners, which was to improve the surveillance and early detection of STDs, was not attained. This case illustrates the pitfalls of having collaboration initiated by passionate professionals, who are only interested in the problem as such, rather than the process. The thirteen disparities between the work cycles of policy, practice and research were not consciously considered. Almost all practical strategies applied in this case were related to the content of the problem, whereas practical strategies to promote the collaboration process were ignored. The small team of passionate practitioners and researchers carried out the work, yielding benefits within their own niche, but without reporting the benefits of the collaboration between practice and research to colleagues and the project management. To colleagues, the collaboration passed unnoticed, maintaining the niche separation for the majority of policy makers, practitioners and researchers. The team considered the actual research and the production of the article as the fulfilment of their responsibility. They went through steps 1 to 4 of the research work cycle. The management of the STD clinic originally intended to feed these research findings into step 1 of the practice work cycle, to investigate the feasibility of surveillance improvements in practice, and subsequently to link these practical findings to step 1 of the policy work cycle so as to adjust surveillance policy and content of refresher courses for doctors. However, as the first work cycle was in progress, the head of the STD clinic accepted a new job, leaving the Maastricht university hospital. The responsibility for the quality improvement of the surveillance was left to unspecified others.

As in the case of problem youths, this project failed to connect collaboration at the individual level to collaboration at the institutional and administrative levels. We may conclude that without a coalition between the partners at the administrative and institutional levels, either informal in terms of an alliance or formalized, the work of well-meaning and enthusiastic professionals will not progress beyond amateurism. 


\section{CHAPTER 5}

\subsection{CASE 3. INTRODUCTION}

\section{Effects of extended opening hours of gambling centres on problem gambling}

The ambition behind this 1991 collaborative project, initiated by the policy domain, was to develop a comprehensive approach in which gambling would not merely be regarded as a problem of individuals but as a public health problem which needs a population-based approach, using laws, regulations, prevention, early detection and treatment.

In the Netherlands, the law on gambling was revised in 1964, stipulating that gambling machines outside casinos have to be authorized by the local authorities. In 1986, money-remitting (rather than games-remitting) gambling machines were legalized, which resulted in an increase in the number of gambling machines, and was followed by signs of an alarming increase in problem gambling. The availability of gambling opportunities is an important issue affecting problem gambling, which in turn may be associated with criminality jeopardizing public safety. Opportunities like low-threshold locations (e.g., sports club canteens, snack bars, community centres) were considered more hazardous especially for the risk group of young people. In 1991, the Maastricht town council accepted the comprehensive policy proposal to ban gambling machines from these low-threshold locations, despite conflicting political views, power tactics and media reports of protests by representatives of the machine gambling industry, sports canteens, community centres and snack bars about unfair economic treatment ${ }^{284}$. The 1991 policy revision was successful and many other local authorities copied the Maastricht '1-0-0' option (i.e., 1 gambling machine permitted in pubs, 0 in snack bars and 0 in sports canteens, community centres and the like). The result was that the number of moneyremitting gambling machines in the town of Maastricht fell from 550 to 370, 200 of which were located in amusement centres which are only accessible to those over the age of $18^{285}$.

In 1999, the Maastricht amusement centres asked the local government for permission to change their closing times from $11 \mathrm{pm}$ to $1 \mathrm{am}$. The policy domain asked for an intersectoral working group to be formed to analyse the effect of such an extension of gambling opportunities. Although the study yielded some indications for an increased prevalence of problem gambling, as discussed in the article included below, the local authorities accepted the extended opening hours. In the context of a rapid expansion of the gambling industry, the growing pressure by the industry on local governments in favour of economic benefits and greater revenues, evidence-based gambling policy is needed. The balance between public health interest, economic gains, public safety and op- 
portunities for leisure activities is a delicate one, as became clear from a proposal to build a sports stadium and covering the funding by extra gambling opportunities 286 , and the recent proposal to open a casino in Maastricht for economic reasons ${ }^{287,288}$. 


\section{CHAPTER 5}

Submitted to: Substance Use and Misuse; date of submission: 18 July 2006

Effects of extended opening hours of gambling centres on problem gambling

Maria W.J. Jansen, MSc, senior adviser, GGD Zuid Limburg, the Netherlands Ronald A. Knibbe, Professor of Social Epidemiology of Alcohol and Drug Use, Maastricht University, CAPHRI, the Netherlands

Hans van Oers, Professor of Public Health, National Institute of Public Health and the Environment (RIVM), the Netherlands

Gerjo Kok, Professor of Applied Psychology, Maastricht University, the Netherlands 


\begin{abstract}
This observational before-after study addresses the effect of extended opening hours at gambling centres in the community on a) the prevalence of problem gambling among low frequency gamblers; and b) the distribution of risk factors among low-frequency visitors. Problem gambling was measured, using a selfadministered questionnaire, on two occasions, namely shortly after opening hours were extended from 11 p.m. to 1 a.m. (T0); and twelve weeks later (T1). The self-administered questionnaire measured risk factors, risk behaviours and components derived from the South Oaks Gambling Screen (SOGS).
\end{abstract}

At T0, 158 gamblers with a mean age of 35.8 years participated. At T1, 167 gamblers with a mean age of 29.9 years participated. On both occasions, the majority of respondents were male. The results show that the prevalence of problem gambling increased significantly between T0 and T1 from $22.1 \%$ to $31.7 \%$. Risk behaviour and risk factors did not change significantly between $\mathrm{T} 0$ and T1. The following factors seemed to contribute to a higher risk profile: a) playing more than one machine simultaneously; b) playing for long periods of time; c) increased spending; and d) alcohol abuse. It is possible that the increase in problem gambling can be partly explained by the larger number of male participants at $\mathrm{T} 1$.

Despite some limitations, this study shows a remarkable increase in the prevalence of problem gambling between the $\mathrm{T} 0$ and $\mathrm{T} 1$ and thus demonstrates that increasing environmental factors like the availability and accessibility of gambling centres does indeed have impact on the prevalence of problem gambling.

keywords: problem gambling, prevalence, gambling opportunity, risk factors, local policy

\title{
INTRODUCTION
}

During the last three decades, gambling problems have increased tremendously on a world-wide scale. Consequently, gambling has become an important public health issue in numerous countries and communities ${ }^{1-3}$. Most countries implement policy and laws on gambling that reflect the public health risks of gambling in that country ${ }^{4}$. In the Netherlands, the 1964 gambling law stipulated that all gambling opportunities involving prize competitions and gambling machines located outside casinos had to be authorised by the local authorities. Since then, machine-based gambling has increased more than any other type of gambling, thus placing the most of the responsibility for prevention of problem gambling in the hands of the local authorities. Money-remitting, rather than gamesremitting gambling machines were legalised in $1986^{5}$ and in 2000 , at the request 


\section{CHAPTER 5}

of local authorities, new legislation on gambling was passed. This new legislation prohibits the placement of gambling machines in low-threshold locations, such as sport club cafeterias, snack bars and community centres ${ }^{6}$. Local governments can, however, grant permits for the establishment of what is termed 'gambling centres', which are amusement arcades that have gambling machines on site. Because of this, the local government is able to impose restrictions on opening hours and oblige gambling centres to contribute to the prevention of problem gambling through efforts such as the distribution of brochures on the risks of gambling.

In 1999, a company owning two gambling centres in the urban community of Maastricht, the Netherlands requested permission to extend opening hours so that these centres close at $1 \mathrm{a}$ a.m. rather than $11 \mathrm{p} . \mathrm{m}$. This article presents a study on the effects of extended opening hours on problem gambling in the community and the number of persons at risk for gambling problems. Using a twelve week follow-up study, the effect of increased gambling opportunities on problem gambling was appraised. This kind of study is of significant importance not only because gambling itself is a problem but also because of a potential correlation between gambling and criminality and because of the fact that young people are highly vulnerable with respect to the development of machine gambling problems $4,7,8$. As a result, the availability of gambling opportunities is an important issue for local policy-makers.

To date, no randomised controlled trials demonstrating the effects of the availability of gambling opportunities on problem gambling and risk behaviour have been conducted. There are however indications that the availability of gambling opportunities does promote gambling behaviour and pathology. Between 1986 and 1991, when money-remitting gambling machines were allowed in the Netherlands, 75,000 money-remitting gambling machines were installed ${ }^{9-}$ ${ }^{11}$. In the same period, the number of gamblers enrolling in treatment programmes for gambling problems increased more than 15-fold from 400 in 1986 to 6814 in $1994^{12-14}$. As a result of restrictions applied by local authorities, the number of gambling machines available declined between 1991 and 1997, from 75,000 to 38,900 . Since 1997 , the number of gambling machines available has remained more or less stable ${ }^{15-18}$ and the incidence of gamblers requiring treatment has decreased. In 1999, 4400 gamblers were enrolled in treatment programs. This number decreased to 2900 in 200319-21.

Previous research has demonstrated the effects of the availability of gambling opportunities on gambling problems. One follow-up study, using pre-test and post-test measurements, demonstrated that the opening of a casino in the U.S. city Niagara Falls resulted in a significant increase in problem gambling ${ }^{22}$, 23. Welte and colleagues ${ }^{24}$ have demonstrated that the presence of a casino within a 10 mile radius of a respondent's home is positively related to problem and pathological gambling, especially for respondents living in disadvantaged 
neighbourhoods. Additionally, the impact of deregulation of riverboat gambling was analysed in a study by Nichols ${ }^{25}$. This study indicated that deregulation results in increased profits for casinos and that the increased profits are partly due to increased spending by gamblers. Govoni et al. ${ }^{26}$ have shown that the opening of a new casino in an area with many legalised gaming venues does not yield any significant differences in problem gambling. However, their study did identify a small number of gamblers that had increased their gambling expenditures. Additionally, a study by Volberg ${ }^{27}$ compared US regions with less than 10 and more than 20 years of legalised gambling and found a higher prevalence of pathological gambling $(1.5 \%$ vs $0.5 \%)$ in regions with a longer history of legalised gambling. In short, numerous studies including the above-mentioned ones describe a consistent relationship between gambling legalisation, increased opportunity to gamble and increased participation in gambling $28-30$.

A specific feature of gambling machines is the rather rapid onset of pathological gambling, regardless of gender, age and comorbidity with illnesses such as drug abuse and psychiatric disorders ${ }^{31,32}$. The main reason for the rapid onset is the existence of intermittent reinforcement on a short interval schedule between input and outcome. This is characteristic to short odds gambling $29,33-35$. Additionally, gambling centres are low threshold venues for young adults, who are particularly vulnerable with respect to the development of problem gambling and exhibit more risk-taking behaviour than other groups. This is particularly true for individuals in lower income categories $3,24,36-39$.

\section{RESEARCH QUESTION}

In 1999, the company owning both gambling centres in Maastricht requested permission to extend opening hours so that the centres would stay open until 1 a.m. instead of 11 p.m. The city council approved the extension of opening hours for a trial period of time, on the condition that the potential negative consequences of extended opening hours be explored in a study that pays special attention to the effect of the extended hours on the gambling behaviour of young people in the Maastricht community. As a result, this study was developed. To our knowledge, no exploratory studies of the effect of extended opening hours on problem gambling had, at that time, been conducted. Due to the fact that time restraints made it impossible to implement a completely optimal study, wherein gambling centres with no extended opening hours in other cities could be used as a comparison setting, a choice was made to focus on low frequency visitors ( $\leq 2$ visits per week) of the gambling centres. By conducting measurements with low frequency visitors, we were able to increase the likelihood that the sample groups were independent of each other. 


\section{CHAPTER 5}

The primary research question of our study was, What is the effect of extended opening hours at gambling centres on the prevalence of problem gambling among low-frequency visitors?

We hypothesized that the extension of opening hours impacts the development of problem gambling in numerous ways. Firstly, extended opening hours may increase frequency or length of time devoted to gambling among gamblers, thereby leading to an increase in associated problems. Secondly, gamblers at risk for the development of a gambling problem may actually become problem gamblers as a result of increased gambling opportunities. Thirdly, new categories of people, who may or may not be at risk for gambling problems, may be attracted to the gambling centres as a result of the extended hours. The gambling centres are located in high traffic areas and it is possible that new visitors will be attracted to the centres on route to or from pubs. Despite the fact that no alcohol is served at the gambling centres, it is possible that extended opening hours can attract visitors that have consumed substantial amounts of alcohol prior to entering the gambling centre, which may result in increasingly frequent visits and more risky gambling behaviour with high financial losses. As a result of this possibility, we choose to not only investigate the effect of extended opening hours on a) the prevalence of problem gambling; and b) changes in risk factors, such as age, sex, and ethnicity; but also on c) the distribution of proximal risk factors, such as sensation seeking and playing more than one machine simultaneously. Consequently, we proposed a second research question, namely: What is the effect of extended opening hours on the distribution of risk factors and risk behaviours among low frequency visitors?

\section{STUDY DESIGN AND PARTICIPANTS}

As practical and ethical restraints made it impossible to conduct a randomized controlled trial, we opted for a observational before-after design (repeated survey), with assessments at the start of the trial period for extended opening hours (T0) and 12 weeks later (T1). As the extension of opening hours was permitted on a trial basis, changes in opening hours were not publicised or advertised.

At T0, the sampling frame consisted of two groups: all those visiting the gambling centres between 9 and 11 p.m. and all those visiting them between 11 p.m. and 1 a.m. At T1, the sample included visitors present during both time slots. In order to ensure independent samples, individuals assessed at T0 were excluded at T1. The only inclusion criterion applied was the ability to speak and write Dutch.

Following recruitment, participants were asked to provide informed consent and were promised a small reward in the form of a gift. The target number 
of participants was 200 at baseline (T0) and 200 at follow-up (T1). We also endeavoured to have participants distributed equally over the two time slots, namely 9 to 11 p.m. and 11 p.m. to 1 a.m. ( $\mathrm{n}=100$ for each period). For both $\mathrm{T} 0$ and $\mathrm{T} 1$, recruitment stopped after the $200^{\text {th }}$ participant agreed to take part in the study. The response rate at T0 was $59.9 \%$ and estimated to be about the same at T1, based on participatory observations by the researchers. Since all those who were interviewed at $\mathrm{T} 0$ had to be excluded at T1, more individuals had to be approached $(n=494)$ before the required net sample of 200 respondents was reached. In order to eliminate selection bias, non-responders were systematically observed. Participatory observation showed that those unwilling to participate (non-responders) were more often part of an ethnic minority (mostly Asian) and/or compulsive gamblers, as evidenced by a nervous attitude and frequent visits of long duration.

\section{MEASUREMENT}

Problem gambling cannot be defined in an absolute or quantitative manner. Rather, problem gambling falls somewhere on a continuum which ranges from recreational gambling to pathological gambling. Whereas pathological gambling is diagnosed using clinical DSM-III-R symptoms, problem gambling is characterised by a high investment of time and money on gambling, resulting in negative changes in one's interactions with one's social environment and a decrease in self-respect ${ }^{38,40-43}$. A self-administered questionnaire, completed at the gambling centres, was used to assess problem gambling. The questionnaire consisted of 58 multiple choice and yes/no items on subjective general health, motivation to gamble, risk factors, risk behaviour and demographic characteristics, such as sex, age, marital status, education, occupation and household composition. Items included are reflected in table 1. Additionally, a motivation scale consisting of ten items was included. The motivation scale showed a reliability of 0.63 for the total sample of high and low frequency visitors and 0.58 for the subsample of low frequency visitors. We consider this acceptable. Further, items that measure alcohol use and drug use a) over the respondent's lifetime; and b) during the last four weeks were included to investigate the potential association between substance abuse and problem gambling. Lastly, three questions were added to the questionnaire at T1. These questions related to a) the duration of gambling sessions; b) the time of day at which gambling began (particularly whether gambling started later at night than before the change in opening hours); and c) changes in spending on gambling following the implementation of extended opening hours.

Problem gambling was assessed using a translated version of the most widely used instrument for measuring problem gambling, namely the South 


\section{CHAPTER 5}

Oaks Gambling Screen (SOGS). The SOGS is a 20-item questionnaire based on DSM-III and DSM-III-R diagnostic criteria. Respondents are required to provide 'yes' or 'no' responses to items and the items are scored by adding up the total number of 'yes' responses. Respondents who provide a 'yes' response on more than five items are considered to suffer from problem gambling (cutoff point). The SOGS is assumed to approximate true population values on problem gambling because no 'gold standard' is available'. Although the SOGS has been shown to be both valid and reliable ${ }^{44,45}$, some concerns do exist with respect to the application of the SOGS in prevalence studies,36-50. In our study, we considered the SOGS to be indeed a valid and reliable measure of problem gambling for the following reasons. With respect to validity, research has shown that the accuracy of the SOGS instrument is greater when applied to a sample with a high base rate of pathological gambling $3,48,51$. Given that the instrument was used for a group of high-risk adults over 18 years of age visiting gambling centres, we assume that the base rate of problem gambling in the visitors of the gambling centre is higher than that of the general adult population, thereby increasing the validity of the instrument. With respect to reliability, we found reliability scores of 0.84 for the total population of high and low frequency visitors and 0.81 for the subsample of low frequency visitors. We also consider this to be acceptable.

\section{ANALYSIS}

We restricted our analyses to low frequency visitors, defined as individuals who visit either or both of the two gambling centres in the community less than twice a week, because frequent visitors were underrepresented at T1. This was a logical consequence of our decision to exclude all respondents who participated at T0 from the assessment at T1. In this fashion, we were able to create independent and comparable sample groups. Our analyses show that the variable low frequency $(\leq 2$ times a week) correlates highly with the variable gambling less than 5 hours a week at all gambling venues, including the gambling centres and other gambling machines in pubs (Pearson's $r=.05 ; \mathrm{p}<.001$ ).

Research question one was investigated by assessing both changes in group composition and the contribution of these changes to problem gambling among low frequency visitors. Using Chi square and non-parametric MannWhitney $U$ tests for the two independent samples, respectively, the prevalence of scoring $\geq 5$ on the SOGS and the group changes in the mean score of the SOGS between T0 and T1 were determined. Whether more distal variables such as changes in socio-demographic characteristics and health composition are contributing factors to changes in problem gambling was also tested using linear regression. Multiple linear regression analyses using extended opening 
hours as the primary independent variable and the mean SOGS score as the dependent variable were performed for the following distal factors: sex (male/female), age ( $<$ or $\geq 40 \mathrm{yrs}$ ), marital status (married: yes/no), occupation, parenthood (have children: yes/no), educational level (low/intermediate level or higher education), ethnicity (native Dutch: yes/no) and subjective general health (positive/negative). For these analyses, all distal factors were transformed to binary variables.

In order to sufficiently investigate research question two, it was imperative that we determined which risk factors and behaviours could potentially cause changes between T0 and T1. To do this, we conducted logistic multivariate regression analyses with the dichotomized SOGS score $(\geq 5$ vs $<5$ 'yes' responses) as the dependent variable for the T0 sample $(n=200)$. In doing this, we assumed that there was no selection bias in the T0 sample. Those risk factors and behaviours which appeared to significantly contribute to problem gambling in low frequency gamblers $(\mathrm{n}=325)$ in the above analysis were applied in a subsequent logistic regression analysis. In this analysis, extended opening hours functioned as the independent variable and the relevant risk factors and behaviours derived from the initial analysis were used as the dependent variables, controlling for age, sex and ethnicity. Differences in risk factors and behaviour between $\mathrm{T} 0$ and $\mathrm{T} 1$ were interpreted as indicative for an effect of extended opening hours at $\mathrm{p}<0.1$, and as significant at $\mathrm{p}<0.05$. The three questions on risk behaviours added at T1 were analysed using linear regression, with the SOGS score as the dependent variable and age, sex and ethnicity as covariates.

\section{RESULTS}

When we compare the prevalence of problem gambling at T0 $(22.1 \%)$ and T1 $(31.7 \%)$, a significant increase is found $(\mathrm{p}<.05)$. Furthermore, despite the fact that the change in the mean SOGS score between T0 (2.84) and T1 (3.13) is not statistically significant, we do see a trend towards an increase in severity.

Table 1 shows that there were significant differences between $\mathrm{T} 0$ and $\mathrm{T} 1$ in terms of age (younger at T1), sex (more males at T1), marital status (more singles at T1), parenthood (more persons without children at T1), and current enrolment in an education programme (higher at T1) (see table 1). 


\section{CHAPTER 5}

Table 1 Characteristics of low-frequent visitors at T0 and T1 $(n=325)$

\begin{tabular}{|c|c|c|}
\hline & $\begin{array}{l}\text { T0 } \\
(n=158)\end{array}$ & $\begin{array}{l}\text { T1 } \\
(n=167)\end{array}$ \\
\hline prevalence $\%$ SOGS $\geq 5$ & 22.1 & $31.7^{*}$ \\
\hline mean SOGS-score & 2.84 & 3.13 \\
\hline $\begin{array}{ll}\text { age: } & \text { mean (yrs) } \\
& \text { median (yrs) }\end{array}$ & $\begin{array}{l}35.8 \\
34\end{array}$ & $\begin{array}{l}29.9^{* * *} \\
27\end{array}$ \\
\hline male $\%$ & 70 & $81,4^{* *}$ \\
\hline married \% & 37.8 & $20.2 * * *$ \\
\hline lives with family $\%$ & 71.7 & 66.4 \\
\hline has children $\%$ & 41.0 & $25.9 * *$ \\
\hline migrant $\%$ & 10.2 & 12.0 \\
\hline low to medium level of education $\%$ & 84.5 & 76.0 \\
\hline unemployed \% & 22.5 & 24.7 \\
\hline currently enrolled in an education programme & 14.6 & $36.1 * * *$ \\
\hline feels healthy $\%$ & 88.5 & 88.6 \\
\hline (almost) always gambles till closing time $\%$ & 18 & $5 * * *$ \\
\hline $\begin{array}{l}\text { (almost) always breaks promise to spend no } \\
\text { more than amount specified beforehand } \%\end{array}$ & 21 & $9 *$ \\
\hline plays at $>1$ machine simultaneously $\%$ & 39 & $54 * *$ \\
\hline
\end{tabular}

Table 1 also shows that the only risk factor that increased between T0 and T1 was 'playing more than one machine simultaneously'. Additionally, when we compare T1 to T0, we see that, at T1, there were fewer visitors that gambled until closing time and fewer visitors who almost always broke their promise to spend no more than an amount specified beforehand.

Comparing marital status at $\mathrm{T} 0$ and $\mathrm{T} 1$, we see that the group assessed at T1 was comprised of more young, unmarried students than the group at T0. Whether this difference in group composition contributed to problem gambling was analysed by conducting a linear regression that controlled for distal factors. The regression coefficient for extended opening hours was 0.082 (n.s.) (see table 2). It is also important to note that males, those who considered themselves to be less healthy than others, and visitors belonging to ethnic minorities scored significantly higher on the SOGS-scale, regardless of the extended opening hours. Additionally, age and marital status did not contribute to problem gambling (see table 2). 
Table 2 Factors related to problem gambling among low-frequent visitors of gambling centres ( $\mathrm{n}=325)$; (SOGS 1-20)

\begin{tabular}{|c|c|}
\hline & Regression coefficient \\
\hline extended opening hours $(0=$ no $)$ & 0.082 \\
\hline age $(0=>40$ yrs $)$ & 0.846 \\
\hline $\operatorname{sex}(0=$ female $)$ & $1.286^{* *}$ \\
\hline marital status $(0=$ married $)$ & 0.393 \\
\hline lives with family $(0=$ yes $)$ & 0.480 \\
\hline has children $(0=$ yes $)$ & 0.398 \\
\hline ethnicity $(0=$ Dutch $)$ & $1.158^{*}$ \\
\hline education level $(0=$ high $)$ & 0.473 \\
\hline employment $(0=$ yes $)$ & 0.034 \\
\hline currently enrolled in an education programme $(0=$ yes $)$ & 0.573 \\
\hline feels healthy $(0=$ yes $)$ & $1.364^{*}$ \\
\hline
\end{tabular}

Table 3 shows the difference in risk factors and risk behaviour between problem $(S O G S \geq 5)$ and non-problem gamblers $(S O G S<5)$ at T0 and the effect of extended opening hours on these risk factors. As expected, problem and nonproblem gamblers differed significantly on almost all recorded risk behaviours and risk factors. In short, increased risk behaviours and risk factors clearly predict problem gambling. Following this conclusion, we applied logistic regression to the sample of low frequency visitors to see whether the extended opening hours influenced risk behaviour and risk factors. Once controlled for age, sex and ethnicity, the analyses indicated that no risk behaviours or risk factors changed significantly between T0 and T1. Factors indicative $(0.1>\mathrm{p}>0.05)$ of an increase in the risk profile were a) alcohol abuse ( $>21$ glasses a week, $\mathrm{OR}=1.8)$; and $b)$ playing more than one machine simultaneously $(\mathrm{OR}=1.5)$. 


\section{CHAPTER 5}

Table 3 Odds ratio's for gambling risk behaviour and risk factors related to problem gambling and related to extended opening hours.

\begin{tabular}{|c|c|c|c|}
\hline Characteristics & $\begin{array}{l}\text { Odds Ratio } \\
\text { problem gam- } \\
\text { bling } \\
(n=200)\end{array}$ & $95 \% \mathrm{CI}$ & $\begin{array}{l}\text { Odds Ratio } \\
\text { extended opening } \\
(n=325)\end{array}$ \\
\hline feels healthy $(0=$ yes $)$ & $3.65^{* *}$ & $1.41-9.43$ & ns \\
\hline consciously planned visit $(0=$ yes $)$ & $0.47^{*}$ & $0.23-0.96$ & ns \\
\hline gambles $>10$ hours a wk $(0=$ no $)$ & $0.16^{* * *}$ & $0.06-0.44$ & ns \\
\hline gambles alone $(0=$ no $)$ & ns & & ns \\
\hline $\begin{array}{l}\text { (almost) always gambles till closing } \\
\text { time }(0=\text { no })\end{array}$ & ns & & ns \\
\hline $\begin{array}{l}\text { (almost) always breaks promise to } \\
\text { spend no more than amount speci- } \\
\text { fied beforehand } \%\end{array}$ & $10.16^{* * *}$ & $2.71-38.05$ & ns \\
\hline $\begin{array}{l}\text { loses more money than is affordable } \\
\text { every week }(0=\text { no })\end{array}$ & $11.75^{* * *}$ & $3.71-37.14$ & ns \\
\hline $\begin{array}{l}\text { Borrows from someone else in } \\
\text { order to gamble }(0=\text { no })\end{array}$ & $10.54^{* * *}$ & $3.63-30.59$ & ns \\
\hline $\begin{array}{l}\text { (almost) always uses start labels } \\
(0=\text { no })\end{array}$ & $3.07 * *$ & $1.48-6.36$ & ns \\
\hline $\begin{array}{l}\text { (almost) always uses a coin storage } \\
\text { bin }(0=\text { no })\end{array}$ & $3.26 * *$ & $1.50-7.09$ & ns \\
\hline $\begin{array}{l}\text { plays at }>1 \text { machine simultaneously } \\
(0=\text { no })\end{array}$ & $5.49 * * *$ & $2.50-12.02$ & $1.55(\mathrm{p}<0.1)$ \\
\hline $\begin{array}{l}\text { withdraws cash from ATM on } \\
\text { location on more than half of the } \\
\text { visits }(0=\text { no })\end{array}$ & $2.87 * *$ & $1.39-5.95$ & ns \\
\hline total motivation score $(0-10)$ & $1.98^{* * *}$ & $1.53-2.56$ & ns \\
\hline $\begin{array}{l}\text { drinks alcohol }>21 \text { glasses } / \text { wk } \\
(0=\text { no })\end{array}$ & ns & & $1.79(\mathrm{p}<0.1)$ \\
\hline lifetime drug use $(0=$ no $)$ & $2.71^{*}$ & $1.25-5.85$ & ns \\
\hline
\end{tabular}

Further analyses indicated that the average gamble time per visit decreased after opening hours were extended. These results are derived from the questions 'At what time did you start today?', and 'At what time do you expect to stop today?' These questions were included both at T0 and T1. However, 14\% of the low frequency visitors did report a longer stay since the introduction of extended opening hours. This was measured by the following questions: 'Do you play for longer periods at a time since opening hours were extended?', and 'Do you start your playing later since opening hours were extended?'. Obviously, these questions were only posed at T1. We believe that the differences in response on 
questions about today's expected length of stay are likely to be coincidental. Furthermore, the intended time to stop may not correspond with the actual time at which gamblers stopped. Although low-frequency gamblers were less likely to stay until closing time after the opening hours were extended, there are indications that the extension of opening hours did lead to an increase in the time and money spent at the gambling centres. Such increases in time (regression coefficient: 2.6; $\mathrm{p}<.001$ ) and money (regression coefficient: $4.3 ; \mathrm{p}<.001$ ) are related to problem gambling. The largest amount of money lost increased from 300 at $\mathrm{T} 0$ to 383 euros at T1 (n.s.).

\section{DISCUSSION}

\section{Discussion of the study design}

The purpose of the present study was to determine whether extending opening hours has an impact on the prevalence of problem gambling among low frequency visitors. We conclude that this is the case.

Despite the overall success of the study, some limitations can be identified. First and foremost, our study design was not optimal. It is widely accepted that the most valid study design for this kind of study is an experimental design using a cohort with randomised assignment of subjects or gambling centres ${ }^{52-55}$. However, this was neither possible nor appropriate as we found the inclusion of newly recruited visitors following the extension of opening hours to be highly relevant and a cohort study would disallow this option. The second best option would have been a time series study with repeated pre-tests and posttests. Unfortunately, we were unable to do this. In our particular study, we were unable to conduct individual follow-up measurements as the amusement arcades company disallowed this. They felt that an individual follow-up may result in embarrassment and feelings of shame about gambling. Evidently, this could pose negative financial consequences for the company and, logically, the company would not allow us to do anything that could potentially decrease their income. Additionally, individual follow-up has significant potential for selection bias and poor retention. This is a serious methodological problem we choose to avoid. Additionally, our study experienced limitations common to all field experiments, such as practical problems, randomisation problems and uncontrolled sources in the context of the wider environment ${ }^{56}$.

An additional limitation was this study's lack of a control group. Given the time restraints applied by the city council, it was impossible to find and include amusement arcades that could function as a control group. The dates for pilot testing the extended opening hours had already been set (from 1 June until 1 September), and expected seasonal effects made the amusement arcades company unwilling to postpone this. The local government also implied a sense of 


\section{CHAPTER 5}

urgency. As a result, we carried out this observational before-after study without a control group. However, despite this limitation, we were able to compile relevant regional data on problem gambling among visitors of the local amusement arcades.

A further limitation of this study is related to response characteristics of our study design. These characteristics served to complicate both the data analyses and the interpretation of the analyses. T0 was in July/August, while T1 was in October/November, making seasonal effects a potential confounder. Additionally, the exclusion at $\mathrm{T} 1$ of visitors who had already responded at T0 resulted in a selective sample at T1, and this obliged us to confine our conclusions to low frequency visitors. Further, little precise information on the total numbers of visitors in either time period is available. However, based on information provided by the research assistants who conducted the fieldwork, we assume that these numbers corresponded with all visitors who were asked to participate (334 at T0 and 494 at T1).

With respect to criticisms of the SOGS instrument, Shaffer \& $\mathrm{Korn}^{3}$ have proposed a universal system for reporting prevalence rates that reflects the underlying continuum of gambling from level 0 (non-gamblers), through level 1 (not experiencing any gambling problems) and level 2 (experiencing subclinical levels of gambling problems), to level 3 (meeting the diagnostic criteria for having a gambling disorder). In accordance with Ladouceur et al.48, we may assume an overestimation of $23 \%$ for problem and probable pathological gambling. Taking this into account, our findings still show prevalence rates of $18 \%$ at T0 and $26 \%$ at T1. Even when we use a SOGS score of 10 as a cut-off value for pathological gambling, our study still shows a prevalence of $3.1 \%$ for pathological gambling within our sample.

Despite the mentioned limitations, we do believe that the present study has produced relevant indicators of the effect of extended opening hours at gambling centres on problem gambling and its risk factors and risk behaviour. However, we recommend more extensive research so that the specific effects of gambling opportunities can be demonstrated.

\section{Discussion of results}

The results indicate that indeed the prevalence of problem gambling among low frequency visitors increased after opening hours were extended. This increase can be partially, but not fully, explained by an increase in visitors with distal or proximal risk factors. However, the extended opening hours did appear to attract a different audience, namely younger, unmarried male students. In general, young, unmarried men are more prone to problem gambling than others $29,57-61$. Although the sample at T1 consisted of significantly more unmarried, younger men enrolled in an education programme than the sample at T0, the only variable that contributed to an increase in SOGS score was sex. However, when we 
look at the differences between $\mathrm{T} 0$ and $\mathrm{T} 1$, we can see that the demographic shift reflects the important role of the extended hours in the temptation to gamble. Pleasure-seekers and young male students visiting pubs and consuming alcohol are likely to seek excitement ${ }^{36}$. A gambling centre that is open during the hours in which pubs are visited and alcohol is consumed is thus highly tempting.

With respect to research question 2, there were indications that extended opening hours do lead to an increase in a) risk of gambling at more than one machine simultaneously; b) alcohol abuse; and c) greater expenditure of time and money at the gambling centre. It is important to note that when individuals begin to experience gambling as an exciting activity, the likelihood that they will engage in it to excess increases ${ }^{36}$. A study among Australian club gamblers compiled a gambler profile that shared certain features with features found in our study. Specifically, the aspect of playing more than one machine simultaneously was consistent in both our study and the study conducted by Hing and Breen $^{58}$. Additionally, the co-occurrence of problem gambling and alcohol abuse found in our study corresponds with other studies on comorbidity $3,19,21,28,62-67$.

Although the 12 week intervention period is useful for identifying changes in risk factors, and, specifically, heavy drinking and playing more machines at once, the time frame was too short to establish a definite and strong correlation between extended opening hours and gambling risk factors and risk behaviour. It is simply impossible to properly identify the development of habitual risk behaviour, not to mention the development of pathological or problem gambling, in just three short months. However, with respect to financial expenditures, our study did show that the largest sum of money lost to gambling increased after the opening hours were extended.

In addition to our findings specifically related to the effect of extended opening hours on problem gambling, our study yielded some general findings on the prevalence of problem gambling among low frequency gamblers visiting amusement arcades in Maastricht. The prevalence rates of $22 \%$ at $\mathrm{T} 0$ and $32 \%$ at T1 (mean 27.1) are remarkably high. It is unlikely that this finding is attributable to bias as the use of self-reported gambling behaviour has proved reliable and valid in earlier studies on problem and pathological gamblers in the USA, Canada, Europe, Australia, New Zealand and elsewhere $3,48,68$. Although the findings of these studies are not fully comparable to our findings due to differences in assessment, it is important to note that most of these nationally representative studies have found lifetime prevalence rates varying from $1.3 \%$ to $7 \%$ among the adult population and $34.9 \%$ to $15.5 \%$ among youths $33,30,39,47,61,64,69,70$. The rates for patients in treatment or inmates in prisons are estimated to reach $15.4 \%{ }^{3,62}$. Furthermore, among subgroups in gambling centres like Australian club gamblers, the prevalence rate for probable pathological gambling was 


\section{CHAPTER 5}

$2.4 \% 58$. Other comparable data on visitors of gambling centres are not available, as far as we know. However, our data do suggest that the prevalence of problem gambling in Maastricht is reasonably high.

The high prevalence rate found in our study raises important questions about the effectiveness of preventive measures implemented in gambling centres. These preventive measures include written brochures and early detection of problem gambling by specially trained staff. Additionally, a ban on those who show serious gambling pathology exists in the form of a blacklist. Unfortunately, this measure is without sanctions. Based on the regional figures about addiction treatment, we found that only 10 of the 130 Maastricht problem gamblers were in treatment and that we do not know if the treatment provided was specifically geared at a gambling problem. Unfortunately, the majority of problem gamblers do not seek treatment and thus remain undetected, despite serious pathology $27,28,47,71$. Based on this study and the high prevalence found in this study, we strongly recommend a significant increase in prevention efforts and early detection of problem gambling at gambling centres. Because the local government grants permits for the establishment of gambling centres, it may make demands on preventive measurements. For example, to provide tailored treatment for problem gamblers, to oblige special training for personnel so that they can identify and confront problem gamblers, to ban the placement of automatic cash machines inside gambling centres, to implement a rule that disallows visitors from playing more than one machine simultaneously, to discontinue the supply of coins storage bins and to disallow start labels.

Our study is, to the best of our knowledge, the first study providing descriptive data on the link between extended opening hours and problem gambling and risk behaviour of gambling centre visitors within a community. Obviously, an increase in gambling opportunities by means of an increase in the number of gambling centres may, like an increase in opening hours, also have an impact on the prevalence of problem gambling. This can be deduced from looking at the consequences of the restrictive Dutch machine gambling policy implemented in 2000 which is now enforced by a model of public policy for gambling opportunities. This regulatory policy resulted in a stabilisation of the number of problem gamblers entering treatment. Clearly, there is a need for more extensive research, not only on the availability of gambling opportunities, but also on gambling problems and vulnerable segments of the population like low socio-economic groups, women, ethno-cultural groups, visitors of gambling centres and casinos, youth, and individuals with subclinical psychiatric disorders and/or addiction. Such research could serve to fulfil public health goals and assist both policy makers and health practitioners in minimising the negative impact of gambling in the community. 


\section{Acknowledgments}

The authors thank research assistants Daniel Boon and Koen Dols, who conducted the fieldwork and initial analyses for this study as part of their internship for Mental Health Studies at the University of Maastricht's Faculty of Health Sciences. We also thank Gerard van Breukelen and Martien van Dongen at the University of Maastricht for their statistical assistance.

\section{References}

1. Korn DA, Shaffer HJ. Gambling and the health of the public: adopting a public health perspective. J Gambl Stud 1999 Winter;15(4):289-365.

2. Messerlian C, Derevensky J, Gupta R. Youth gambling problems: a public health perspective. Health Promot Int 2005 Mar;20(1):69-79.

3. Shaffer HJ, Korn DA. Gambling and related mental disorders: a public health analysis. Annu Rev Public Health 2002;23:171-212.

4. Van 't Veer A, Moerland H, Fijnaut C. Gokken in drievoud. Facetten van deelname, aanbod en regulering. Gouda: Quint, 1993.

5. Schuurman J, Jordens K. Nederlandse Staatswetten nr. 69, Wet op de Kansspelen. Den Haag: Staatsuitgeverij, 1988.

6. Nederlandse Staatscourant. Speelautomatenregeling 2000. Nederlandse Staatscourant. 2000(105):10-8.

7. Op het Veld G, De Leijer L, De Vos T. Preventie en voorlichting. In: De Vos T, editor. Gokverslaving: hulpverlening en preventie. Lisse: Swets \& Zeitlinger Publishers, 1995.

8. Van den Heuvel M, Van Kalmthout J. Gokverslaving en criminaliteit in Nederland. Een verkennende studie naar de aard en de omvang van aan gokken en gokverslaving gerelateerde criminaliteit. Tilburg: Katholieke Universiteit Brabant, Vakgroep Vrijetijdwetenschappen, 1995.

9. Ministerie van Justitie. Gelijke Kansen. Eindrapport van de Commissie Coördinatie en Harmonisatie Kansspelbeleid. Den Haag: Ministerie van Justitie, 1992.

10. Mutsaers HPM, Gijsen E. Onderzoek gemeentelijk beleid speelautomaten; verslag eerste fase - nulpuntmeting. Den Haag: SGBO Onderzoeks- en Adviesbureau van de VNG, 1992.

11. Nederlands Meetinstituut. Jaarrapportage 1991 kansspelen. Delft: Nederlands Meetinstituut; 1992.

12. Hermkens PLJ, Kok KM. Kansspelen in Nederland: ontwikkelingen, deelname en risicoaspecten. Tijdschrift voor Alcohol, Drugs en andere Psychotrope Stoffen 1988;14:162-72.

13. Janssens R, Driessen P, Overbekking J. Gokken in Nijmegen en Deventer. Onderzoek naar omvang, aard en ontwikkeling van gokgedrag uitgevoerd in opdracht van de gemeenten $\mathrm{Ni}$ jmegen en Deventer. Deventer-Nijmegen: Gemeenten, 1989.

14. Kok KM, Hermkens PLJ, Van Wijngaarden JJ, Van de Wijngaart GF. De kast verslaan. In opdracht van Vereniging Automatenhandel Nederland. Utrecht: Universiteit van Utrecht, 1991.

15. Mutsaers HPM. Het iets-nul-nul-beleid: landelijk onderzoek gemeentelijk beleid speelautomaten in 1995, vergelijkingen met 1992 en 1993. Den Haag: SGBO Onderzoek \& Advies; 1995.

16. Mutsaers HPM. Gemeenten hebben al ingezet: landelijk onderzoek gemeentelijk beleid speelautomaten in 1997; vergelijkingen met 1992, 1993 en 1995. Den Haag SGBO; 1997.

17. SGBO. Gemeenten hebben al ingezet. Landelijk onderzoek gemeentelijk beleid speelautomaten in 1997; vergelijkingen met 1992, 1993 en 1995. Den Haag: SGBO, 1997. 


\section{CHAPTER 5}

18. Van den Broek H, Zandhuis E, De Vos T. Handreiking gemeentelijk speelautomatenbeleid. Den Haag: VNG, 1999.

19. LADIS. Landelijk Alcohol en Drugs Informatiesysteem. http://www.ivv.nl; 1988-1995.

20. Nederlandse Vereniging van CAD's. Jaarstatistieken uit Landelijk Alcohol en Drugs Informatiesysteem 1991 en 1992. Utrecht: Nederlandse Vereniging van CAD’s, 1992.

21. Ouwehand W. Kerncijfers LADIS 1995-2003. Utrecht: Nationaal Alcohol en Drugs Informatiesysteem, http://www.ivv.nl, 2003.

22. Room R, Turner NE, Ialomiteanu A. Community effects of the opening of the Niagara casino. Addiction 1999 Oct;94(10):1449-66.

23. Turner N, Ialomiteanu A, Room R. Checkered expectations: Predictors of approval of opening a casino in the Niagara community. J Gambl Stud 1999 Spring;15(1):45-70.

24. Welte JW, Wieczorek WF, Barnes GM, Tidwell MC, Hoffman JH. The relationship of ecological and geographic factors to gambling behavior and pathology. J Gambl Stud 2004 Winter;20(4):405-23.

25. Nichols MW. Deregulation and cross-border substitution in Iowa's riverboat gambling industry. J Gambl Stud 1998 Summer;14(2):151-72.

26. Govoni R, Frisch GR, Rupcich N, Getty H. First year impacts of casino gambling in a Community. J Gambl Stud 1998 Winter;14(4):347-58.

27. Volberg RA. The prevalence and demographics of pathological gamblers: implications for public health. Am J Public Health 1994 Feb;84(2):237-41.

28. Abbott MW, Williams MM, Volberg RA. A prospective study of problem and regular nonproblem gamblers living in the community. Subst Use Misuse 2004 May;39(6):855-84.

29. Blaszczynski A, Huynh S, Dumlao VJ, Farrell E. Problem gambling within a Chinese speaking community. J Gambl Stud 1998 Winter;14(4):359-80.

30. Petry NM, Armentano C. Prevalence, assessment, and treatment of pathological gambling: a review. Psychiatr Serv 1999 Aug;50(8):1021-7.

31. Breen RB, Zimmerman M. Rapid onset of pathological gambling in machine gamblers. J Gambl Stud 2002 Spring;18(1):31-43.

32. Gotestam KG, Johansson A. Characteristics of gambling and problematic gambling in the Norwegian context: a DSM-IV-based telephone interview study. Addict Behav 2003 JanFeb;28(1):189-97.

33. Bohmers W. Ambulante gedragstherapeutische behandeling van gokproblemen. In: Bohmers W, ed. Handboek verslaving, hulpverlening en beleid. Houten: Bohn Stafleu Van Loghum, 1994; p. 3-24.

34. De Vos A, Oberg LJF. Crimineel gedrag in relatie tot gokken. Tijdschrift voor Alcohol, Drugs en andere Psychotrope Stoffen 1988;14:207-11.

35. Eckblad GF, Von der Lippe AL. Norwegian lottery winners: cautious realists. J Gambl Stud 1994;10:305-23.

36. DiClemente CC, Story M, Murray K. On a roll: the process of initiation and cessation of problem gambling among adolescents. J Gambl Stud 2000 Autumn;16(2-3):289-313.

37. Orford J. Low income and vulnerability for gambling problems. Addiction 2004 Oct;99(10):1356.

38. Prins MCJ. Problematisch gokken: Een verkennend onderzoek naar de achtergrondsituatie van 47 gokkers. Amsterdam: Jellinek, 1987.

39. Gray PB. Evolutionary and cross-cultural perspectives on gambling. J Gambl Stud 2004 Winter;20(4):347-71.

40. Hermkens PLJ, Becker HA, Kok KM, Mutsaers HPM. Kansspelen als riskante gewoonte (KARGO): Probleemaspecten in Nederland verkend. Utrecht: Rijksuniversiteit Utrecht., 1988.

41. Kusyszin I. Compulsive gambling, the problem definition. Int J Addict 1978;13:19-34. 
42. Prins MCJ. Gokverslaving. Patient Care 1989;13:50-6.

43. Ten Den C, Bieleman B, De Bie E. Zorgen dat het een spel blijft. Groningen/Rotterdam: Intraval, buro voor onderzoek en advies, 1993.

44. American Psychiatric Association. Diagnostic and Statistical Manual of Mental Disorders; 4th ed. Washington: American Psychiatric Association, 1994.

45. Lesieur HR, Blume SB. The South Oaks Gambling Screen (SOGS): a new instrument for the identification of pathological gamblers. Am J Psychiatry 1987 Sep;144(9):1184-8.

46. Battersby MW, Thomas LJ, Tolchard B, Esterman A. The South Oaks Gambling Screen: a review with reference to Australian use. J Gambl Stud 2002 Fall;18(3):257-71.

47. Derevensky JL, Gupta R, Winters K. Prevalence rates of youth gambling problems: are the current rates inflated? J Gambl Stud 2003 Winter;19(4):405-25.

48. Ladouceur R, Bouchard C, Rheaume N, Jacques C, Ferland F, Leblond J, et al. Is the SOGS an accurate measure of pathological gambling among children, adolescents and adults? J Gambl Stud 2000 Spring;16(1):1-24.

49. Langhinrichsen-Rohling J, Rohling ML, Rohde P, Seeley JR. The SOGS-RA vs the MAGS7: prevalence estimates and classification congruence. J Gambl Stud 2004 Fall;20(3):259-81.

50. Strong DR, Lesieur HR, Breen RB, Stinchfield R, Lejuez CW. Using a Rasch model to examine the utility of the South Oaks Gambling Screen across clinical and community samples. Addict Behav 2004 May;29(3):465-81.

51. Strong DR, Breen RB, Lesieur HR, Lejuez CW. Using the Rasch model to evaluate the South Oaks Gambling Screen for use with nonpathological gamblers. Addict Behav 2003 Oct;28(8):1465-72.

52. Bouter LM, Van Dongen MCJM, Zielhuis GA. Epidemiologisch onderzoek; opzet en interpretatie. Houten: Bohn Stafleu van Loghum, 2005.

53. Campbell DT, Stanley JC. Experimental and quasi-experimental designs for research. Boston, MA: Houghton Miffin, 1996.

54. Polit DF, Hungler BP. Nursing Research. Principles and methods. Philadelphia: J.B. Lippincott Company, 1991.

55. Swanborn PG. Sociaal-wetenschappelijk onderzoek en de samenleving In: Swanborn PG, ed. Methoden van sociaal-wetenschappelijk onderzoek. Meppel: Boom, 1994; p. 377-411.

56. Lipsey MW, Cordray DS. Evaluation methods for social intervention. Annu Rev Psychol 2000;51:345-75.

57. Desai RA, Maciejewski PK, Dausey DJ, Caldarone BJ, Potenza MN. Health correlates of recreational gambling in older adults. Am J Psychiatry 2004 Sep;161(9):1672-9.

58. Hing N, Breen H. A profile of gaming machine players in clubs in Sydney, Australia. J Gambl Stud 2002 Summer;18(2):185-205.

59. Kroesbergen HT, Laeijendecker-Burger ML, Meulmeester JF, Wassenaar J. Kansspelautomaten en schoolgaande jongeren; een exploratief onderzoek. T Soc Gezondheidsz 1992;70:729-35.

60. Potenza MN, Fiellin DA, Heninger GR, Rounsaville BJ, Mazure CM. Gambling: an addictive behavior with health and primary care implications. J Gen Intern Med 2002 Sep;17(9):721-32.

61. Lamberton A, Oei TPS. Problem gambling in adults: an overview. Clinical Psychology and Psychotherapy 1997;4:84-104.

62. Henderson MJ. Psychological correlates of comorbid gambling in psychiatric outpatients: a pilot study. Subst Use Misuse 2004 Jul;39(9):1341-52.

63. Ibanez A, Blanco C, Donahue E, Lesieur HR, Perez de Castro I, Fernandez-Piqueras J, et al. Psychiatric comorbidity in pathological gamblers seeking treatment. Am J Psychiatry 2001 Oct;158(10):1733-5. 


\section{CHAPTER 5}

64. Potenza MN, Kosten TR, Rounsaville BJ. Pathological gambling. JAMA 2001 Jul 11;286(2):141-4.

65. Spunt B. Pathological gambling and substance misuse. Subst Use Misuse 2002 JunAug;37(8-10):1299-304.

66. Spunt B, Dupont I, Lesieur H, Liberty HJ, Hunt D. Pathological gambling and substance misuse: a review of the literature. Subst Use Misuse 1998 Nov;33(13):2535-60.

67. Welte JW, Barnes GM, Wieczorek WF, Tidwell MC. Simultaneous drinking and gambling: a risk factor for pathological gambling. Subst Use Misuse 2004 Jul;39(9):1405-22.

68. Hodgins DC, Makarchuk K. Trusting problem gamblers: reliability and validity of selfreported gambling behavior. Psychol Addict Behav 2003 Sep;17(3):244-8.

69. Welte JW, Barnes GM, Wieczorek WF, Tidwell MC, Parker J. Gambling participation in the U.S.--results from a national survey. J Gambl Stud 2002 Winter;18(4):313-37.

70. Wiebe JM, Cox BJ, Mehmel BG. The South Oaks Gambling Screen Revised for Adolescents (SOGS-RA): further psychometric findings from a community sample. J Gambl Stud 2000 Autumn;16(2-3):275-88.

71. Nathan PE. The role of natural recovery in alcoholism and pathological gambling. J Gambl Stud 2003 Fall;19(3):279-86. 


\section{CASE 3. OVERVIEW OF APPLIED PRACTICAL STRATEGIES}

\section{The administrative level}

In 1991, the Maastricht local government appointed an intersectoral steering committee to develop its gambling policy. Partners involved were the alderman responsible for gambling policy, assisted by civil servants, and the police, addiction care service, GGD, the judiciary and the gambling machines trade association (Vereniging Automatenhandel Nederland). At the initiative of the policy domain, a temporary coalition was built, although without formal agreements and without the research domain being represented. In 1999, another temporary and informal coalition was built, again at the initiative of the policy domain, this time including the policy, practice and research domains [coalition building +-]. Since the legalization of money-remitting gambling machines in 1986, the police and judiciary had seen signs of increasing numbers of problem gamblers, and the problem was placed high on the policy agenda, as it jeopardized public safety. In 1999, the policy domain again initiated agenda setting for public safety reasons [agenda setting +]. The Maastricht mayor and aldermen decided to reconsider the current gambling policy and its policy instruments. This initiative meant that the practice domain did not have to go through timeconsuming agenda setting procedures, and a window of opportunity arose. As local government is assumed to be the main influential agenda setter ${ }^{289}$, no practical strategies for advocacy were required in 1991 or in 1999 [advocacy -]. Practical strategies to influence willingness to collaborate were not required either, as the collaboration in 1991 was commissioned by the mayor, ensuing from the regular tripartite consultations (Driehoeksoverleg) between the mayor, the head of police, and the head of the public prosecution service (hoofdofficier van Justitie), and public services tend to comply with requests from these tripartite consultations. In 1999, the relevant alderman asked the GGD to investigate the issue. Consultations by the GGD to involve the research field led to a coalition between the policy, practice and research domains [sidestepping the formal system +-$]$.

In 1991, no additional resources were provided nor requested, because the advisory role was considered to be part of the GGD's regular task and to be an opportunity to influence local public health policy. In 1999, resources were requested to carry out the research [resource negotiations +- ].

\section{The institutional level}

Both in 1991 and 1999, no assessment was made of the collaboration process [diagnosis-]. A road map for activities and interventions relating to the project 


\section{CHAPTER 5}

content was drawn up and approved by the institutional management. Local government was the commissioning party and formulated activities by assigning tasks to the various institutional managers. Tasks for the police consisted of an investigation of criminality due to gambling and public safety problems around gambling centres. The judiciary was to advise on feasible adjustments of regulations and legislation. The GGD was asked to investigate the prevalence of problem gambling and to recommend preventive instruments. In 1999, the GGD asked the Department of Health Promotion (mental health division) of Maastricht University to carry out the research on the prevalence of problem gambling. Institutional managers accepted this task division because it was tailored to the knowledge, expertise and capacity available within each of the institutes. Although the task assignments were content-oriented, their joint implementation resulted in an improved institutional collaboration [activities and interventions +-]. Commitment was not perceived as an issue of debate, because the institutional managers involved are used to complying with orders from the local government, especially when public safety is at stake [commitment -]. A provisional intersectoral management structure was created, which was chaired by the mayor in 1991, and by the head of the municipal Department of Internal Affairs in 1999 [intersectoral management structure +]. Since communication was strictly guided by the regular municipal channels, no special practical strategies were indicated [communication structure -]. Evaluation of the process was not anticipated, and apart from the collaboration process, the policy adjustments were not evaluated either [evaluation -]. Resources were provided by the partners involved, with an extra grant in 1999. Staff were appointed by the institutional managers, who gave them ample time to implement their tasks [resources + ]

Practical strategies to support the working climate were not given explicit attention. The existing working climate in the policy and practice domains, and in 1999 also in the research domain, appeared to produce a productive environment for collaboration and positive relationships, mainly thanks to a longstanding collaboration on local addiction problems [mission statement -], [interviews role-models -]. Internships were proposed by the GGD and agreed upon by the other institutional managers, but they related to the project content only, without being intended to support a culture of collaboration. Two interns from the Department of Health Promotion of the Faculty of Health Sciences of Maastricht University did the research field work [internships +-].

Within the intersectoral working group, the institutional managers stimulated a culture of rendering services to other domains. For example, legal information and literature were exchanged, law texts were 'translated' into practical guidelines and knowledge about gambling addiction was exchanged by means of site visits. Tools were mutually made available, such as administrative data on licenses and police reports about offenders [rendering services, access 
to tools + ], and a formal and informal consultation structure was promoted [formal and informal consultations + ]. The required capacity was discussed within each institution and extra time was made available. GGD capacity was made available to disseminate the Maastricht policy to other GGDs, i.e. dissemination within their own niche [diagnosis and feedback +]. No other practical strategies were applied regarding capacity [recruiting and retaining staff -], [performance indicators and professional development -], [kick-off conference -]. The leading role of local government reflected legitimate leadership, making the appointment of a leader superfluous [sidestepping the formal system -].

\section{The individual level}

A relatively small joint working group was formed by the local administration, the police, the judiciary, the GGD and the addiction care service, which in 1999 was also joined by the Department of Health Promotion of Maastricht University. The members of the working group diagnosed the problem in terms of content and collaboration aspects, and gave regular feedback to each other on both content and collaboration [diagnosis and feedback +]. Group discussions in multidisciplinary groups were common practice and were perceived as very fruitful because the judiciary system, the economy, public order and public health are disciplines which tend to operate separately, and which now exchanged views in the multidisciplinary group [group discussion in multidisciplinary groups +]. Learning by doing was implemented through task assignment and reflection by the chairperson of the working group, who came from the policy domain. This strategy was accepted by the group members and appeared to be effective [learning by doing + ]. A detailed action plan was prepared by the GGD in 1999 only. The group members did not deem such a plan necessary in 1991, as they proceeded step by step, depending on developments within the group in terms of the content of the problem [detailed action plan, formats, materials +-]. Group members or the chairperson did not apply any other practical strategies to enhance attitude, perceived norms, outcome expectations, behavioural capability or self-efficacy to promote collaboration between the niches [feedback by peer networking -], [lectures and discussion -], [master classes with skills training - ].

Publications by practitioners in policy journals linked the policy and practice niches $285,290-294$. The Maastricht alderman participated in national conferences to explain the town's restrictive gambling policy, which was perceived as rather innovative at that time. However, these contributions did not extend beyond the policy domain. Publications in 1999 were restricted to the research domain ${ }^{295}$ [in-service coaching to publish beyond one's own niche +- ]. 


\section{CHAPTER 5}

\section{Comments}

The gambling problem was interpreted as relevant by the policy, practice and research domains, and was initiated by the local administration as a result of regular tripartite consultations. The GGD initiated the involvement of the research domain in 1999. Civil servants, researchers and practitioners collaborated in going through steps 1 to 4 of the work cycle, at the request of the local authorities, to provide content-oriented recommendations at certain points in time. Due to time constraints on the part of the policy domain, the research design carried out in 1999 was not exhaustive, which resulted in inconclusive data on the effect of extended opening hours. The results from the research and practice cycles were not fed into step 1 of the policy work cycle in order to monitor and incrementally improve local gambling policy. The initiation of step 1 again required the agreement of stakeholders at the administrative level. However, monitoring the effect of the revised gambling policy was not at issue.

At the individual level, the collaboration was perceived favourably, as partners who engaged in the gambling issue pooled their talents, beliefs and understanding in an effort to draw up the advice they had been requested to provide, and in doing so they were taken seriously by the local authorities. In this perspective, the work cycle differences between the niches at individual level were overcome with relative ease. Roles were clearly defined, access to each other's knowledge was provided without barriers and there was a spirit of cooperation. This spirit was further enhanced during the advisory process in 1991, as the economic and public health viewpoints diverged. The economic viewpoints were supported by the gambling machine trade association, the public health viewpoints by the public health practitioners and policy makers. The final decision to decrease the number of money-remitting gambling machines resulted in a feeling among practitioners and policy makers of having defeated a common opponent.

The collaboration to promote evidence-based public health could have been optimized if more frequent links between the policy, practice and research domains had been created and if the time constraints imposed by the policy domain in 1999 could have been solved. The empirical data in 1999 revealed a high prevalence of problem gambling at the Maastricht gambling centres. However, no links were created with what actually went on inside gambling centres as regards the recommendations for prevention, or with research to assess the effects of these recommendations. The recommendations that were made emphasized the impact of the environmental factors on problem gambling within the centres: prevention and early detection of problem gambling focusing on special training for staff to improve their skills in detecting problem gambling; a ban on cash points inside the gambling centres; rules forbidding customers to play on more than one machine simultaneously at gambling centres and bans 
on coins storage bins and start labels. Although the local government was the only party with the legal authority to oblige gambling centres to implement these recommendations by linking them to the licensing procedure, the local authorities refrained from action, and were not stimulated to act by social or policy entrepreneurs from the research or practice domain either.

In this case, we may conclude that the temporary collaboration between the policy, practice and research domains resulted in policy adjustments in 1991 and 1999, both enacted by the council. The collaboration did not continue beyond the council's decisions, nor in evidence-based improvements by incremental development of local gambling policy. 


\section{CHAPTER 5}

\subsection{CASE 4. INTRODUCTION}

\section{Systematic approach to hepatitis $B$ vaccination programmes for risk groups: a literature study}

In this project, collaboration between the practice, research and policy domains was initiated at the national level, after which it passed down to the local level, and was intended to improve public health by improving hepatitis B vaccination programmes. In low-endemic regions, infections with the hepatitis B virus (HBV) occur predominantly in adults, largely as a result of sexual intercourse and parenteral transmission. Therefore, the HBV control in the Netherlands includes counselling and contact tracing of HBV cases to prevent further transmission, as well as targeted vaccination of high-risk groups and immunization of children born to HBsAg positive mothers detected during pregnancy checks. In 1983, and again in 1996, the Health Council of the Netherlands concluded that vaccination of highrisk groups was unsatisfactory ${ }^{296,297}$. Previous research by the GGD, addiction care service (Mondriaan Zorggroep) and the National Institute for Public Health and the Environment (RIVM) among drug addicts revealed high prevalence rates of hepatitis B infection 298,299 . A pilot project on the feasibility of HBV vaccination among high-risk groups was conducted in the Netherlands between 1998 and the end of 2000, at the request of the Ministry of Health, Welfare and Sport ${ }^{300}$. The pilot was managed and supervised by the National Coordination Centre for Communicable Diseases Outbreak Management (LCI; now called: Centre for Infectious Diseases RIVM). Six pilot regions were selected, based on proposals submitted. Southern Limburg, where the GGD was to cooperate with Maastricht University, was selected as one of the six pilot regions. The national initiative fitted in with the local needs, based on the hepatitis B prevalence rates among drug addicts. During the pilot project, many different outreach strategies were developed to reach the risk groups. In view of the positive effects of the pilot, the ministry decided in 2001 to intensify the HBV vaccination campaign, and GGD participation was expanded. During the diffusion stage (2001-2006), the GGDs received additional funding for implementation. From 2007 onwards, the HBV vaccination will be regarded as one of the routine public health tasks. From the start of the campaign in 1998 until 2005, 42,000 persons at risk were vaccinated. Because the target group is a hidden population, it is not possible to estimate the percentage that has been vaccinated so far ${ }^{301}$.

The dissemination of routine HBV vaccination for groups at risk has been evaluated as successful ${ }^{302}$. The article included below describes the findings of a literature search on the barriers to and facilitators of hepatitis B vaccination among high-risk groups. This study was part of the preparation for the 1998 campaign in the Southern Limburg pilot region, and concluded that a systematic approach was required. 
Published (in Dutch) in Tijdschrift voor Gezondheidswetenschappen 2004; 82:87-96

Systematic approach to hepatitis B vaccination programmes for risk groups: a literature study

M.W.J. Jansen, MSc, senior adviser, GGD Zuid Limburg, the Netherlands H.J. Hospers, Assistant Professor, Maastricht University, Faculty of Psychology, Department of Experimental Psychology, Maastricht, the Netherlands 


\title{
CHAPTER 5
}

\begin{abstract}
Specific risk groups are currently not being adequately reached by hepatitis B vaccination programmes. A survey of the international literature has shown that there has so far been little empirical research into facilitators and barriers to vaccination-related behaviour (i.e. willingness to participate and compliance), the determinants of such behaviour and the relevant environmental variables. Hardly any studies of hepatitis B vaccination have systematically examined whether participation and compliance are affected by the level of exposure to health education messages, the intensity of health education campaigns, attitude, actual or subjectively perceived high-risk behaviour, free-of-charge vaccine provision, perceived reliability of the vaccination service provider and easy access. A planned, systematic approach is a precondition for successful future policies to promote tailored vaccination programmes.
\end{abstract}

Key words: hepatitis $B$ vaccination, risk groups, vaccination-related behaviour

\section{INTRODUCTION}

Current vaccination policies require tailored strategies, and this is even more true for future policies, when new vaccines will become available for specific target groups. Adequately reaching such specific target groups and motivating them to comply requires a thorough knowledge of behavioural determinants and behaviour change. The Health Council of the Netherlands has reported that the traditional hepatitis B risk groups (prostitutes and their clients, intravenous drug users and homosexual and bisexual men) have been inadequately reached with existing policy instruments for the last two to three decades. ${ }^{1,2}$ Based on the favourable results of a pilot project, the Dutch Ministry of Health, Welfare and Sport decided in 2001 to intensify the selective vaccination campaign for these traditional risk groups. ${ }^{3}$ This paper discusses the first four steps of a systematic process to develop a vaccination strategy tailored to the traditional risk groups. It surveys the international literature on the relevant determinants of the effectiveness of vaccination strategies. We do not discuss those risk groups being catered for by institutionalised health care, nor pregnant women.

\section{SYSTEMATIC APPROACH}

Voluntary participation in and completion of vaccination programmes can be promoted using the PRECEDE-PROCEED model (see figure 1), which pro- 
vides a suitable structure for the planning and evaluation of tailored vaccination programmes. ${ }^{4}$ The model offers a stepwise analysis of the problem, the relevant behavioural and environmental factors, determinants, intervention development and finally implementation and evaluation.

Phase 1 of the planning model describes the social consequences of the health problem and assesses quality of life aspects. Hepatitis B is a disease transmitted by sexual and blood contacts. While acute infections with the disease can be lethal $(>1 \%)$, the burden of disease is mainly determined by chronic infections. About $10 \%$ of all hepatitis B infections become chronic, and about half of all chronic infections result in cirrhosis of the liver, liver failure or liver carcinoma. Chronic hepatitis B carriers are potentially infectious to other members of society. Morbidity and mortality rates of chronic infections are high, as are the costs, especially among the traditional risk groups.

Phase 2, the epidemiological assessment, analyses the magnitude of the health problem in greater detail. Until 1999, the annual number of reported new cases of hepatitis B in the Netherlands was about $250 .{ }^{5}$ Cross-sectional seroprevalence research has shown that about $2 \%$ of the Dutch population shows evidence of having gone through an infection, and that $0.2 \%$ are chronic carriers. 6,7 The national register of notifiable sexually transmitted diseases shows that the occurrence of acute hepatitis $\mathrm{B}$ has stabilised in recent years to about 1.5 per 100,000 inhabitants. However, there are indications that acute hepatitis B rates have been rising again since the year 2000. ${ }^{8}$ GP monitoring stations are reporting a 2-6-fold rise in the incidence of acute hepatitis B (with a variance of 3.2-6.1/100,000 inhabitants). ${ }^{9} \mathrm{New}$ infections are three times as likely to occur among men than among women. A routine screening of patients visiting an STD clinic in Rotterdam in 1999 yielded a hepatitis B prevalence of $7 \%: 2.6 \%$ among heterosexuals and $9.8 \%$ among homosexuals and bisexuals. Of the women in this population, $0.7 \%$ were chronic carriers, against $0.6 \%$ of the men. ${ }^{8}$ Research among substance users in the southern region of the Dutch province of Limburg yielded prevalence rates (anti-HBc positive persons) of $63 \%$ in 1996 and $67 \%$ in 1999, and found that $6 \%$ and 4\%, respectively, were chronic carriers (HbsAg positive). ${ }^{10,11}$ Similar studies in Rotterdam and The Hague found prevalence rates of hepatitis B among intravenous drug users of $61 \%$ and $35 \%$, respectively. ${ }^{12,13}$ In a monitoring study among homosexual and bisexual men, $8 \%$ reported ever having had hepatitis $\mathrm{B}$, although this was not clinically verified. ${ }^{14}$

The third phase of the PRECEDE-PROCEED model analyses the contributions of personal and collective behaviour and environmental factors to the health problem. Almost $60 \%$ of infections are caused by sexual transmission: $30 \%$ through homosexual contacts and $29 \%$ through heterosexual contacts, while $21 \%$ are infected by other sources and the infection route is unknown in $20 \%$ of cases. ${ }^{8}$ Hepatitis B infection can be prevented by practising 


\section{CHAPTER 5}

safe sex (using condoms for vaginal, oral and anal sex), safe methods of substance use (by not injecting or by using clean syringes and other injectionrelated materials), good personal hygiene (razor blades, tooth brushes) and vaccination. For the traditional risk groups, vaccination is the most reliable form of prevention, and is also cost-effective. ${ }^{15}$ The monitoring study among homosexual and bisexual men also asked about vaccination status. Two thirds of the men said they had not been vaccinated against hepatitis A or B. Of the $33 \%$ who had been vaccinated, $52 \%$ reported having been vaccinated against hepatitis $\mathrm{B}$, while $35 \%$ did not know whether they had been vaccinated against hepatitis A or B, and 13\% said they had been vaccinated against hepatitis A. ${ }^{14}$

Vaccination behaviour requires a brief effort by the person to be vaccinated, involving his/her willingness to participate (i.e., start the programme) and compliance (completion of the vaccination programme). Whereas willingness to participate is a decisive factor in the extent to which a specific target group can be reached, compliance is essential to achieve optimum immunological response. Vaccination behaviour is different from things like food behaviour, which is to a considerable extent determined by engrained habits and lifestyle, and for which behavioural changes require sustained continuous personal efforts. Another relevant factor is the design of the vaccination programme, which should fit the context of the target group's social environment. Environmental variables that affect both willingness to participate and compliance include costs and the location where vaccination is to take place (in terms of travelling distance, whether the risk group trusts the agency providing the vaccination service, the quality of the agency's staff in terms of expertise and social skills, and privacy guarantees).

The fourth phase analyses the behavioural component, that is, the actual vaccination-related behaviour (see table 1). This analysis can use theoretical models like the Health Belief Model, the Theory of Reasoned Action, the Social Cognitive Theory and the Theory of Planned Behaviour. ${ }^{16}$ Although these models differ in terms of specificity, explicitness and emphasis, they show many similarities. Briefly, these models are based on the idea that human behaviour can be explained by (1) knowledge about and awareness of a particular health problem; (2) attitude; (3) social influences and (4) self-efficacy. ${ }^{17}$ The models also show the discrepancy between a person's intention to engage in a particular behaviour and their actual behaviour, since the person's environment may impose certain barriers.

The actual design of a vaccination programme affects willingness to participate and compliance. A programme may include a pre- and post-vaccination screening (blood sampling). Pre-vaccination screening is intended to establish any existing immunity (from a prior disease episode) or chronic carriership. Since blood sampling is often perceived as stressful by both clients and welfare workers (especially among intravenous substance users) and is time-consuming, 
vaccination is often done without prior screening, or blood is sampled simultaneously with the first vaccination at the initial consultation, in order to stimulate the willingness to participate. The disadvantage of vaccination without prior screening is that the clients cannot be told if they are carriers. This means that carriers leave the consultation with a false sense of security while they can still transmit the disease to others. On the other hand, simultaneous blood sampling and administration of the first vaccination does provide information for follow-up vaccinations and reduces the total number of consultations, which may improve compliance. In the ideal situation, the post-vaccination titre is determined after vaccination, to assess the adequacy of the immune response. The total programme, including assessment of post-vaccination titre, will then take up to 7.5 months, which may affect compliance.

The above analyses allow the further phases in the planning model to be addressed and the intervention to be designed.

Figure 1 The PRECEDE-PROCEED model ${ }^{4}$

\section{PRECEDE}

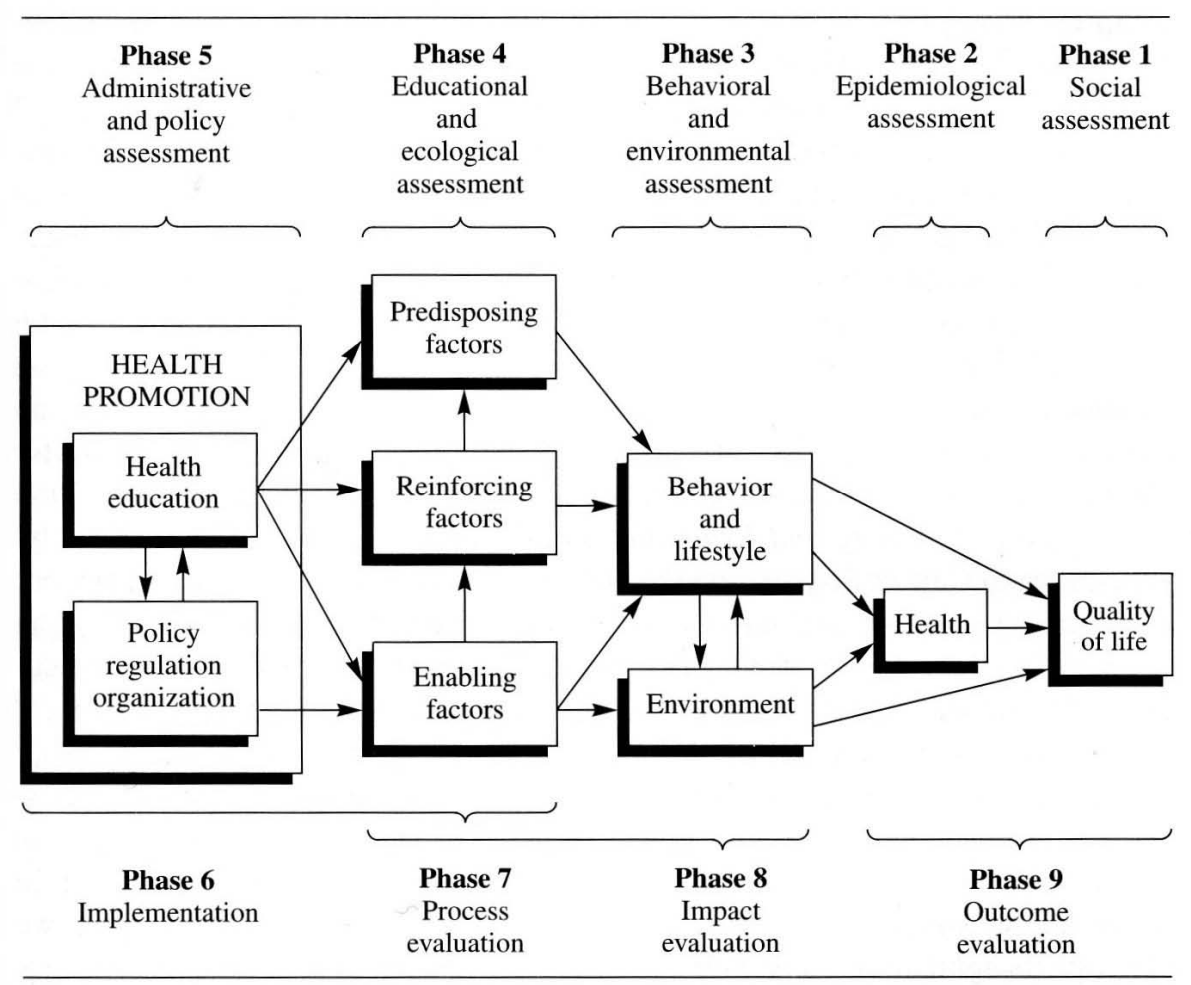

PROCEED 


\section{CHAPTER 5}

\section{METHODS}

The present literature study covered the 1988-2000 period, and searched the Medline, PsycINFO, ERIC and Current Contents databases using the search terms 'compliance', 'adherence' and 'hepatitis B', combined with 'vaccination', 'immunisation' and 'screening'. The lists of references in the resulting articles were screened for other relevant publications. Articles were selected if they reported the number of participants and compliance. One review was added because it provided information on facilitators and barriers. Information on the facilitators and barriers listed in table 1 can only be obtained from comparisons between participants and non-participants or between compliers and noncompliers.

We calculated participation and compliance rates. Participation was expressed as the relative coverage of the at-risk population, i.e., the ratio between the number of participants - minus those not needing vaccination, e.g., because they have already had the disease - and the total at-risk population. Compliance was calculated as the percentage of compliers, i.e., the proportion of fully vaccinated persons among those participants who were susceptible or potentially susceptible to the disease. Participants were defined as actually or potentially susceptible persons (anti-HBc negative or status unknown) who accepted the first vaccination.

\section{RESULTS}

In all, 29 articles met our inclusion criteria. Five articles related to intravenous drug users, three to heterosexuals, four to homosexual and bisexual men, one to prostitutes, four to combinations of the above risk groups, five to student populations, six to adolescents and one to primary school children. Apart from the study of the influence of parents on underage children and the study by Rosenthal, none of the studies examined social influence or self-efficacy as behavioural determinants of willingness to participate and compliance, so we decided not to include these determinants in the description of findings.

\section{Willingness to participate and compliance}

Six of the 28 studies (excluding the review study), i.e. $21 \%$, did not report the total size of the at-risk population or failed to report the numbers of people refusing participation or dropping out, which means that we could not calculate the relative coverage. The remaining 22 studies all involved well-defined populations that were reached through specific channels (e.g., a methadone clinic, school or STD clinic). Ten of the studies found that fewer than $50 \%$ of the 
target group started the vaccination programme, whereas more than $50 \%$ started the programme in 12 of the studies (see table 1).

Eleven studies reported less than $50 \%$ compliance, while 15 reported more than $50 \%$. The lowest compliance rate $(2.4 \%$ for men and $4.1 \%$ for women) was found in a study among heterosexuals, which retrospectively asked patients visiting an STD clinic whether they had been fully vaccinated. The highest compliance rate $(95.6 \%)$ was found among primary school pupils vaccinated against hepatitis B (see table 2).

Forty percent $(\mathrm{n}=4)$ of the studies that reported a high participation rate (i.e., with more than $50 \%$ of the target group participating; $n=11$ ) found low compliance rates $(<50 \%)$ and reported that the objective of the vaccination programme had not been achieved.

\section{Determinants of willingness to participate}

Knowledge and risk awareness

Over half of the studies $(17 / 29=59 \%)$ reported on the intensity of the educational efforts (individual, group education, tailored approach) (see table 3). Knowledge was assessed in six of the studies, one of which compared participants and non-participants. This study showed that knowledge does not affect participation. Nineteen of the studies assessed whether respondents were aware of the risk of hepatitis B (risk indicators, self-reported and/or clinical, risk perception); five of these $(5 / 29=17 \%)$ also studied the differences between participants and non-participants. One study found no difference in high-risk behaviour (which we assumed to reflect risk awareness) between participants and non-participants, but found a high level of high-risk behaviour in both groups. The remaining three studies found a positive association between high-risk behaviour and willingness to participate. One study found no relation between risk perception and willingness to participate.

\section{Attitude}

Attitude was assessed in four of the studies $(4 / 29=14 \%)$, two of which compared participants and non-participants. Both of these studies found a positive effect of attitude, specified as the importance for one's own health of complying with behavioural recommendations to counteract infectious diseases. No relation was found between participation and confidence in the safety of the vaccine. Other 'beliefs' were not assessed in these studies. 


\section{CHAPTER 5}

Table 1 Determinants of willingness to participate in and compliance with hepatitis B vaccination, based on explanatory models of behaviour

\begin{tabular}{|c|c|c|}
\hline & Willingness to participate & Compliance \\
\hline $\begin{array}{l}\text { Knowledge } \\
\text { and risk } \\
\text { awareness }\end{array}$ & $\begin{array}{l}\text { The target group has been informed } \\
\text { of the importance of vaccination; the } \\
\text { target group is aware of the serious- } \\
\text { ness of the illness, its own vulnerabil- } \\
\text { ity and personal high-risk behaviour } \\
\text { (i.e.,what high-risk behaviours relate } \\
\text { to hepatitis B; whether the person is } \\
\text { aware of this; risk perception) }\end{array}$ & $\begin{array}{l}\text { The target group has been informed } \\
\text { about the importance of completing } \\
\text { the vaccination programme; the target } \\
\text { group is aware of the risk of hepatitis } \\
\text { B after incomplete vaccination } \\
\text { ('awareness'; risk perception) }\end{array}$ \\
\hline Attitude & $\begin{array}{l}\text { The target group is convinced of the } \\
\text { advantages of vaccination; the target } \\
\text { group weighs up all relevant short- } \\
\text { and long-term pros and cons (beliefs) } \\
\text { of participating in the vaccination } \\
\text { programme (understanding that it is } \\
\text { important for their health; no fear of } \\
\text { jabs or pain; believing in the safety of } \\
\text { the vaccine; no side effects, no fear of } \\
\text { 'foreign' substances in their blood; } \\
\text { positive attitude towards visiting a } \\
\text { doctor or the vaccination service) }\end{array}$ & $\begin{array}{l}\text { The target group is convinced of the } \\
\text { advantages of compliance; the target } \\
\text { group weighs up all pros and cons of } \\
\text { three consultations, and possibly four } \\
\text { in the case of post-vaccination titre } \\
\text { assessment (same beliefs as for will- } \\
\text { ingness to participate; understanding } \\
\text { that it is important for their health and } \\
\text { necessary for full protection to visit } \\
\text { the service three or four times; main- } \\
\text { taining safe sex behaviour to avoid } \\
\text { other infections; no false sense of } \\
\text { security) }\end{array}$ \\
\hline $\begin{array}{l}\text { Social influ- } \\
\text { ence }\end{array}$ & $\begin{array}{l}\text { The target group is receiving positive } \\
\text { signals and support for participation } \\
\text { from their social environment (opin- } \\
\text { ions of others with whom they iden- } \\
\text { tify; what do others expect of me; } \\
\text { motivation to conform to others' } \\
\text { behaviour; word-of-mouth encour- } \\
\text { agement in their own social environ- } \\
\text { ment) }\end{array}$ & Same as for willingness to participate \\
\hline Self-efficacy & $\begin{array}{l}\text { The target group is positive about } \\
\text { their own ability to participate in the } \\
\text { vaccination programme (owning up } \\
\text { about sexual behaviour or sexuality) }\end{array}$ & $\begin{array}{l}\text { The target group is convinced of their } \\
\text { ability to complete the programme }\end{array}$ \\
\hline Environment & $\begin{array}{l}\text { The target group does not perceive } \\
\text { insurmountable barriers in their envi- } \\
\text { ronment (privacy is ensured; low } \\
\text { threshold access to service; trust in } \\
\text { quality of service; no financial barriers; } \\
\text { no insurmountable barriers in the } \\
\text { vaccination programme) }\end{array}$ & $\begin{array}{l}\text { The target group's environment } \\
\text { stimulates repeat sessions (friendly } \\
\text { treatment; reminders; incentives; } \\
\text { location of vaccination service does } \\
\text { not change between sessions; no } \\
\text { insurmountable problems in vaccina- } \\
\text { tion programme) }\end{array}$ \\
\hline
\end{tabular}




\section{Environment}

Environmental factors assessed in the studies were classified into location (outreaching or not), the effects of vaccine costs, the use of an informed consent procedure (yes/no), the quality of the vaccination service provider (in terms of reliability and employing qualified staff) and other factors. The type of location of the vaccination service was reported in nearly all articles $(28 / 29=97 \%)$. These were usually low-threshold locations or the sites where the risk groups were located. Free-of-charge vaccination was offered in 19 of the studies, 6 of which claimed that costs would mean a barrier to participation, without offering empirical evidence. One study provided empirical evidence for a positive relation between free-of-charge vaccination and participation, while one study found no such effect. Forty-eight percent of the articles (14/29) explicitly mentioned that an informed consent procedure had been used. Ten studies reported on the importance of the quality of the vaccination provider (with reliability of the providing agency mentioned 6 times, qualified staff 5 times and a positive attitude of staff towards traditional risk groups 4 times). None of these compared participants with non-participants.

\section{Determinants of compliance}

\section{Demographic factors}

Nine of the 29 studies (31\%) assessed the relation between age and compliance. Five studies found a positive effect, while 4 found no effect (see table 4).

\section{Knowledge and risk awareness}

Four studies compared compliers and non-compliers in terms of educational level, while two compared them in terms of knowledge about hepatitis B. Three studies found a positive effect of educational level, whereas one found no effect. A positive effect of knowledge was found in both of the studies assessing this factor.

Eleven studies compared compliers and non-compliers in terms of highrisk behaviour (assumed to reflect risk awareness). Three studies found a positive effect of actual high-risk behaviour (HIV positive, STD positive and IDU) on compliance, while two found a negative effect and two found no effect. Risk perception was found to have a positive effect in three studies and no effect in one study, while actual high-risk behaviour had a positive effect in one study, a negative effect in one study and no effect in two studies.

\section{Attitude}

Two studies, one of them being the review study, compared compliers and non-compliers in terms of attitude. The review concluded that there was a positive relation between compliance and the beliefs listed in table 1 . The other 


\section{CHAPTER 5}

study only examined pre-vaccination attitudes towards completing the vaccination programme, and found no relation with compliance.

\section{Environment}

Fifteen of the articles described the reminder system for the second and third vaccinations (6 studies used a letter plus phone call, 4 only used a letter, 1 only used a phone call and 4 used a different method). Three of the studies assessed the effect of the reminder system on compliance; all of them found a positive effect. Five studies used special incentives to promote compliance, but none assessed their effects. Three studies examined the effect of the vaccination schedule on compliance. Two of them found a positive effect of shorter vaccination schedules (0-1-2), whereas one found no effect on compliance. (Shortened vaccination schedules produce a lower immune response.)

\section{DISCUSSION}

This literature review found few studies that systematically examined differences, in terms of behavioural determinants, between participants and nonparticipants of hepatitis B vaccination programmes or between compliers and non-compliers of such programmes. Hardly any analytical research has been done to assess whether these determinants predict willingness to participate or compliance and what interventions have a favourable effect on knowledge, risk awareness and attitude. None of the studies included in this review quantified the degree of exposure to educational efforts among the target group. Only one study assessed the intensity of the educational effort and its effect on participation and compliance, leaving it unclear whether intensive education campaigns stimulate participation and compliance. Only five studies examined whether high-risk behaviour explains the willingness to participate; their results suggest a positive association. Studies of the effect of high-risk behaviour or risk perception on compliance have not yielded unequivocal results. The two studies examining the influence of attitude on participation and compliance did not yield decisive evidence either.

The limited number of methodologically valid studies assessing the effect of certain environmental factors do not allow any definitive conclusions. Offering vaccination free-of-charge appears to have a positive effect on participation when costs are perceived to be high, and reminder systems seem to have a favourable effect on compliance. The potential effects of incentives and the duration of the vaccination programme have not been examined in randomised studies. The perceived reliability of the agency providing the vaccination service would appear to be an important environmental variable. 
Many studies fail to report the size of the target group. Although it is not easy to assess the size of a 'hidden' population, since those who are not reached by a programme do not spontaneously come forward, various methods are available to estimate the size of (local) risk groups, which can be combined to yield a comparatively reliable estimate. We had great difficulty calculating the compliance rates in many studies, since they often failed to report the necessary data or differed too much in their designs. This meant that some studies calculated compliance on the basis of the number of serologically tested persons (sometimes without reporting the number of persons who did not need vaccination because they had had the disease), whereas others based themselves on the number of persons actually coming in for their first vaccination, or on those receiving their first vaccination or their first plus second vaccinations. Two studies asked respondents retrospectively whether they had been fully vaccinated, though considerable doubt remains about the reliability of the answers.

Although our literature review has yielded some information that could be useful in setting up vaccination programmes, many questions about the design of such interventions unfortunately remain unanswered. The general approach is to develop interventions on the basis of the assessments in phases 1-4 of the PRECEDE-PROCEED model, to fit the target group as well as the environmental conditions. A useful instrument to achieve this is the Intervention Mapping (IM) protocol, ${ }^{17}$ which explicitly addresses all steps in the development of an intervention. Numerous interventions for HIV prevention among substance users or those engaging in certain sexual behaviours have been developed using the IM methodology. $18,19,20,21$ Future research into the Dutch situation should involve a much more accurate assessment of the behavioural and environmental determinants of willingness to participate and compliance. Such research with regard to hepatitis $B$ vaccination will need to distinguish certain subgroups, since groups like substance users, prostitutes and homosexual and bisexual men will differ in terms of knowledge, risk awareness, beliefs about attitude and self-efficacy, and social and environmental factors. Since our literature study into hepatitis B vaccination among hard-to-reach groups showed that environmental variables are relatively important, intervention development for these groups should involve a thorough analysis of environmental determinants. Such interventions should also distinguish between the various subgroups among the target group. The IM protocol can indicate how specific health education activities for specific environmental conditions can be derived from general programme objectives. The interventions should be designed in such a way as to maximise the willingness to participate and the compliance, as participation and compliance are crucial elements of vaccination behaviour. High participation rates with low compliance rates are not effective, and the same is true for a combination of low participation and high compliance. 


\section{CHAPTER 5}

Such a thorough process of determinant analysis and intervention development is essential for any future vaccination programme, as this paper has tried to illustrate for the case of hepatitis $B$ vaccination among risk groups. The same recommendation can also be made for other vaccination programmes. In view of the envisaged expansion of the national vaccination programme - including hepatitis B vaccination for children in risk groups and meningococcal vaccination, both of which were already introduced in 2003 - as well as the increasingly critical attitude towards vaccination among the Dutch public and the decreasing vaccination coverage among very young Dutch children, there is a clear need for thorough scientific research into vaccination-related behaviour.

\section{Acknowledgement}

This study was financially supported by the Dutch national pilot project on the feasibility of hepatitis B vaccination among risk groups, which was funded by the Dutch Ministry of Health, Welfare and Sport. 
MULTIPLE CASE STUDY
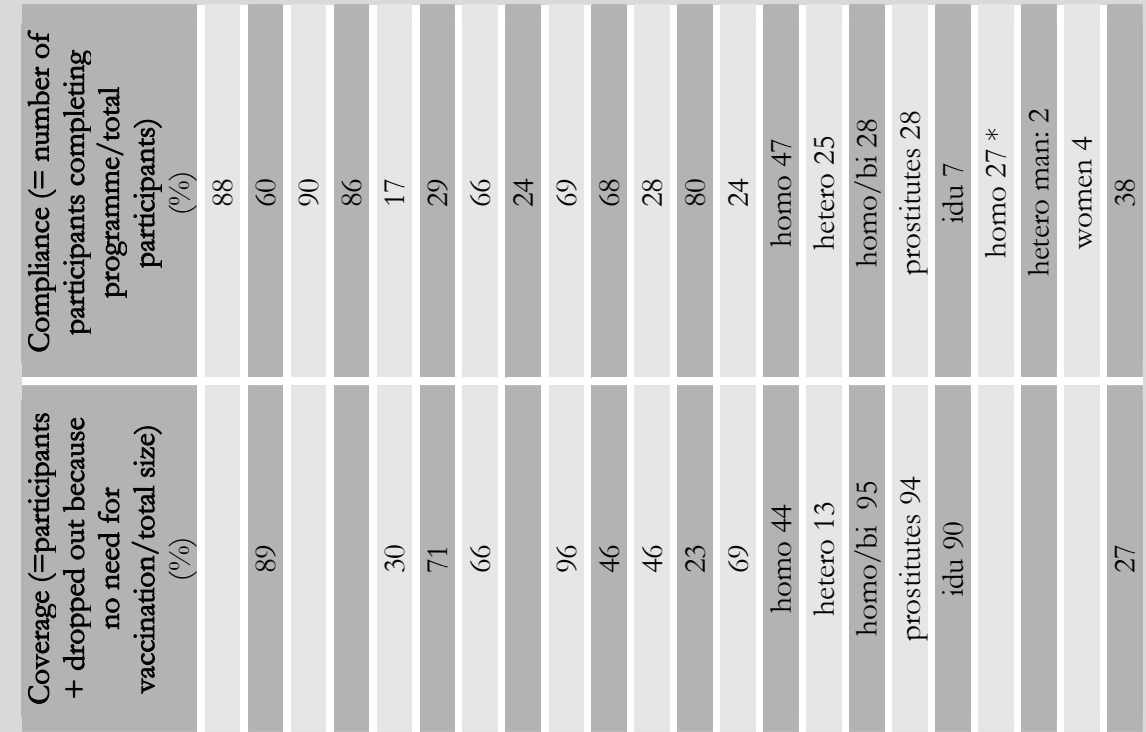

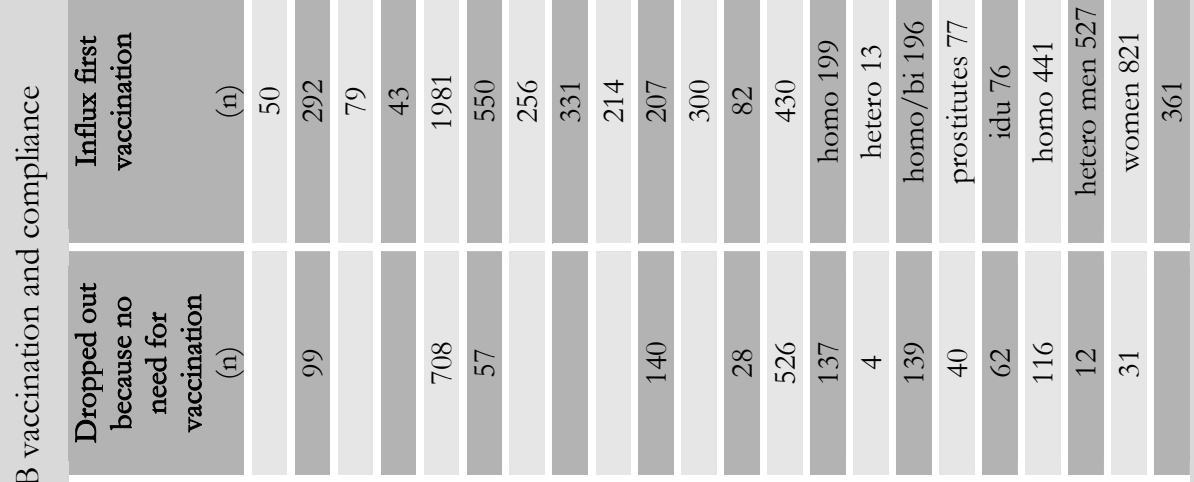

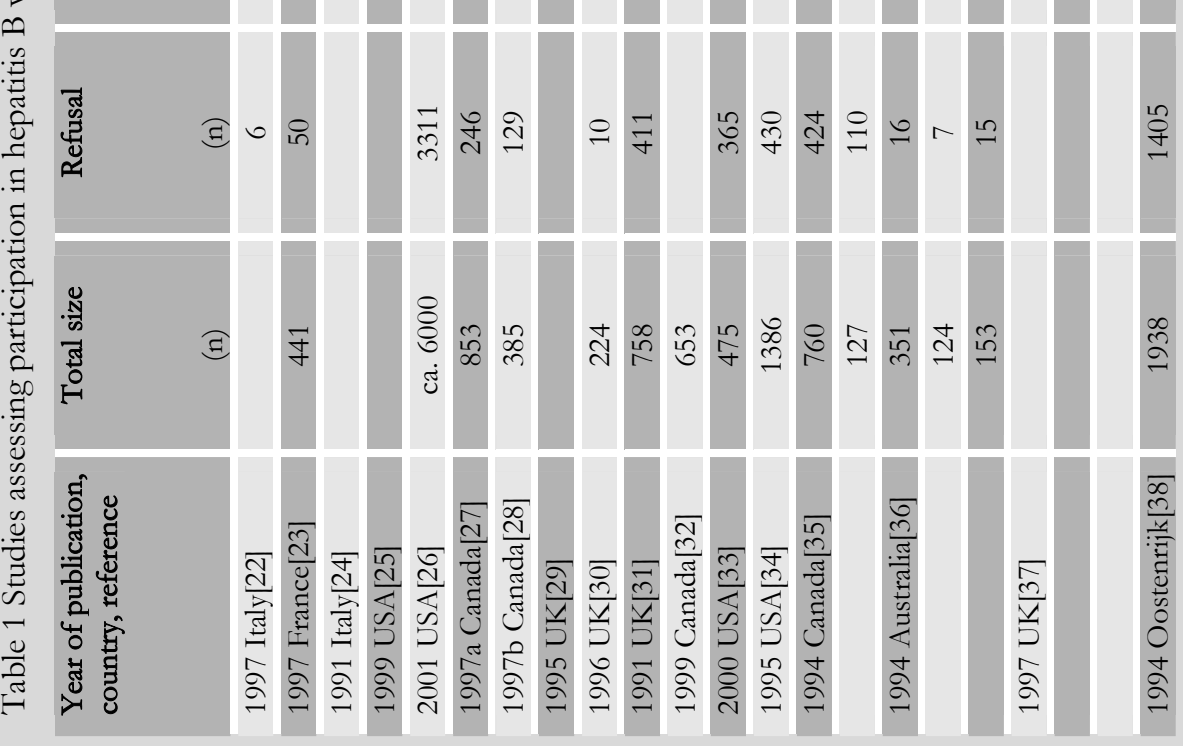


CHAPTER 5

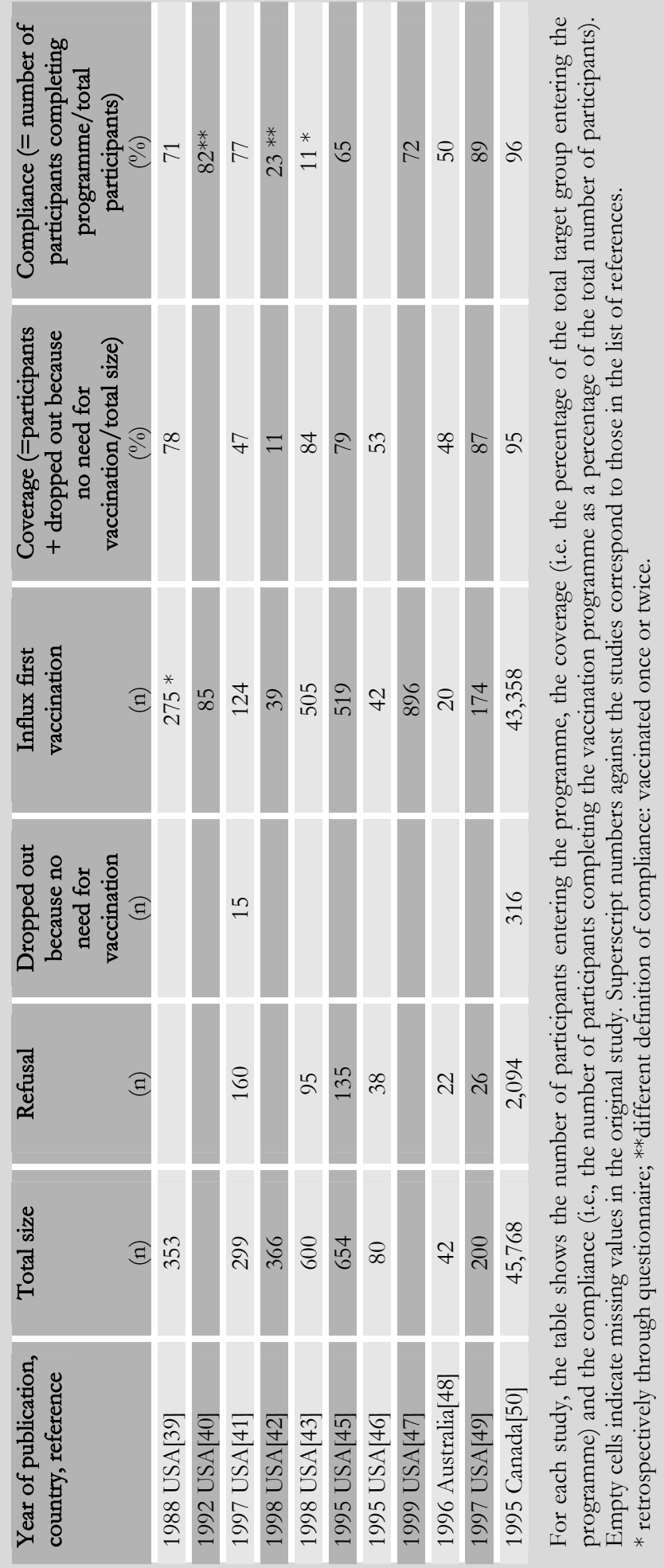

158 
MULTIPLE CASE STUDY
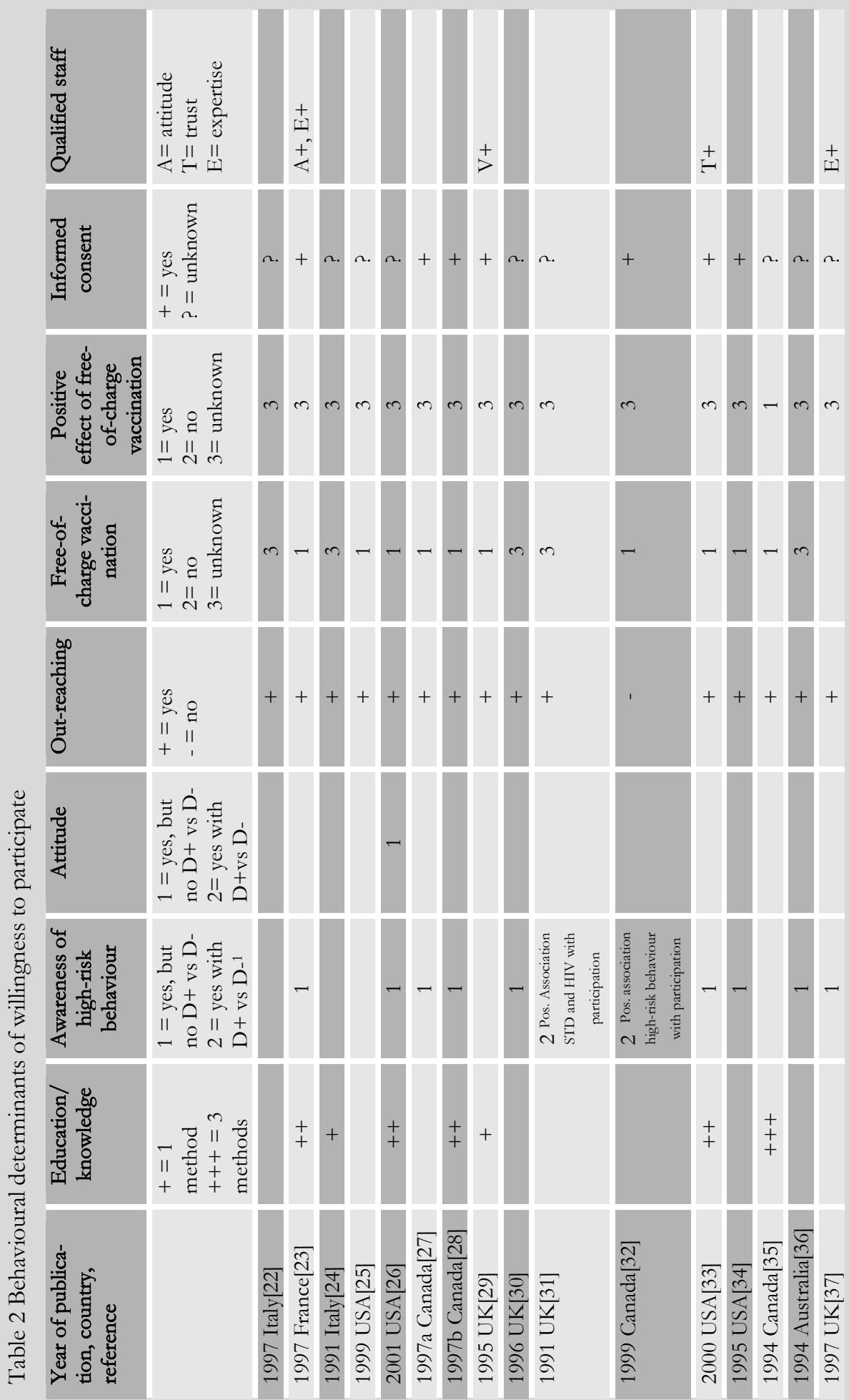
CHAPTER 5

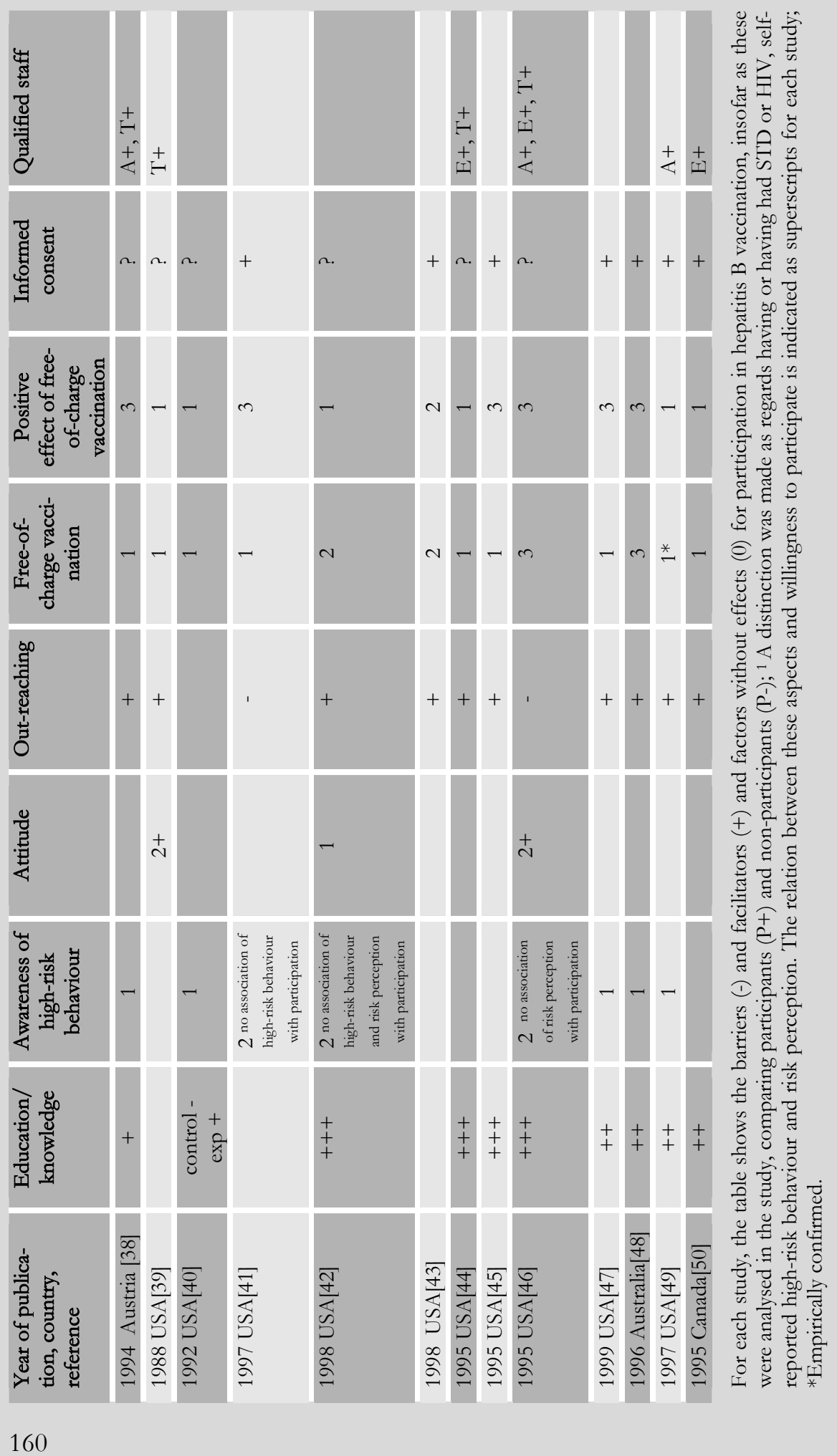


MULTIPLE CASE STUDY
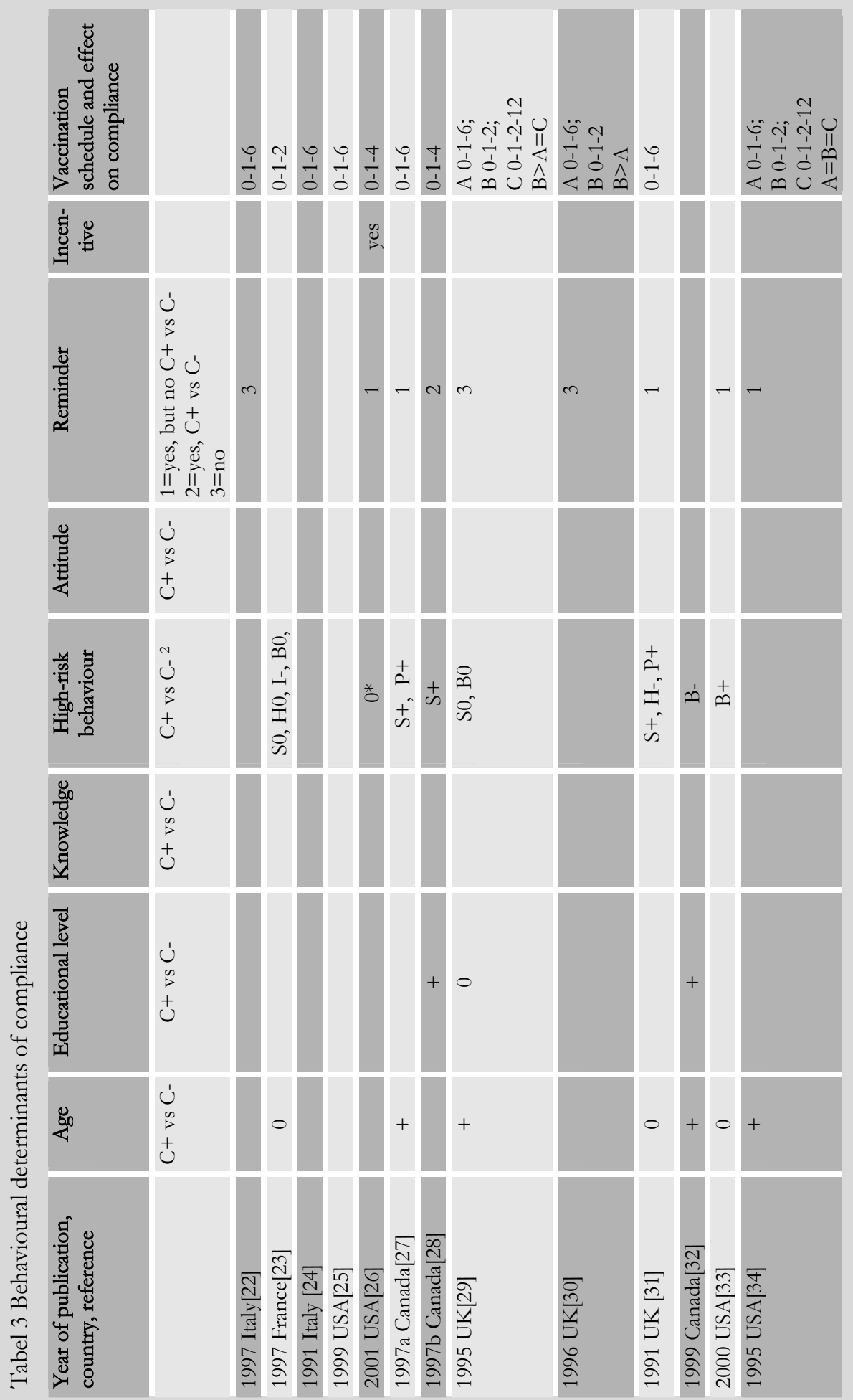
CHAPTER 5

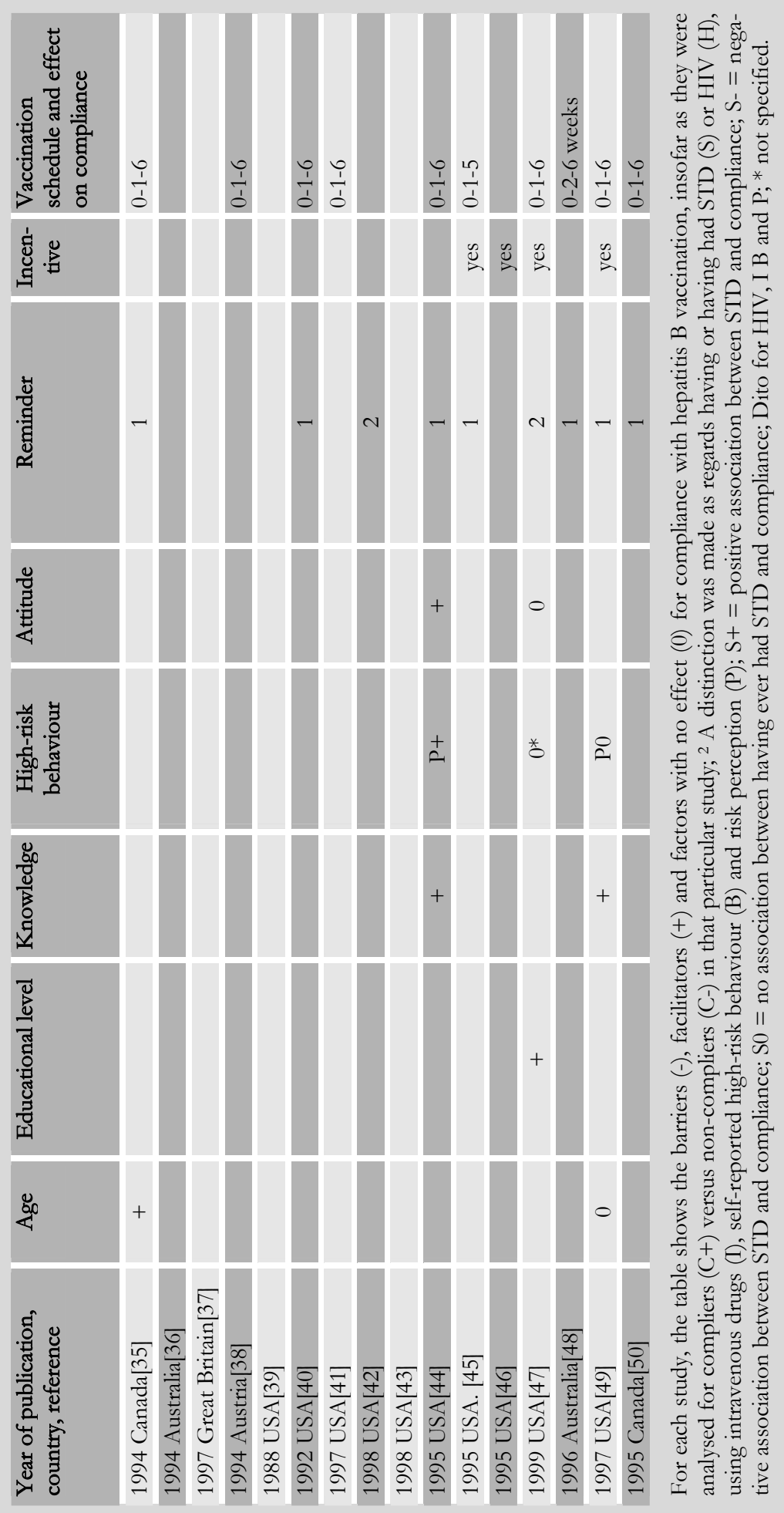

162 


\section{References}

1. Gezondheidsraad. Advies inzake hepatitis B. Rapportnr 1983/22. Den Haag: Staatsuitgeverij 1983.

2. Gezondheidsraad. Commissie Hepatitis B. Bescherming tegen hepatitis B. Publicatienr. 1996/15. Rijswijk: Gezondheidsraad 1996.

3. Steenbergen JE van, Burgt M van der, Waldhober Q. Proefproject haalbaarheid Vaccinatie van Risicogroepen Hepatitis B. Utrecht: Landelijke Coördinatiestructuur Infectieziektenbestrijding 2001.

4. Green LW \& Kreuter MW. Health Promotion and planning: an educational and ecological approach (3rd ed.). Mountain View, CA: Mayfield 1999.

5. Rijlaarsdam J, Smits LJM, Laar MWJ van de. De aangifte van hepatitis B in Nederland in de periode 1976-1998. Infect Bull 1999; 10: 185-6.

6. Marrewijk CM van, Veldhuijzen IK, Conyn-van Spaendonck MAE, et al. Prevalence of hepatitis B viral markers in the Dutch population: a population-based surveillance study (Pienter project). Rapport nr.243680001. Bilthoven: RIVM 1999.

7. Laar MWJ van de, Termorshuizen F. Seksueel Overdraagbare Aandoeningen (SOA). In: VTV deel I. De gezondheidstoestand: een actualisering. Bilthoven: RIVM 1997.

8. Veldhuizen IK, Rijlaarsdam J, Beuker RJ, et al. Het vóórkomen van hepatitis B in Nederland. Infect Bull 2001; 12: 233-7.

9. Bartelds AIM. Virale hepatitis 1994-1997. In: Continue Morbiditeitsregistratie Peilstations Nederland. Jaarverslag 1997. Utrecht: NIVEL 1998.

10. Carsauw HHC, Roozendaal CM van, Scheepens JMFA, et al. Infecties met HIV, HBV en HCV onder injecterende druggebruikers in Heerlen/Maastricht. Bilthoven: RIVM 1997.

11. Beuker RJ, Berns MPH, Rozendaal CM van, et al. Surveillance van HIV-infecties onder injecterende druggebruikers in Nederland: metingen Heerlen/Maastricht 1998/99. Bilthoven: RIVM 2001.

12. Wiessing LG, Toet J, Houweling $\mathrm{H}$, et al. Prevalentie en risicofactoren van HIV-infectie onder druggebruikers in Rotterdam. Rapport nr.213220001. Bilthoven: RIVM 1995.

13. Beuker RJ, Watzeels A, Berns MPH, et al. Surveillance van HIV-infectie onder injecterende druggebruikers in Nederland: meting Den Haag 2000. Bilthoven: RIVM 2001.

14. Hogeweg JA, Hospers HJ. Monitoronderzoek 2000. Maastricht: Universiteit Maastricht 2000.

15. Wit GA de, Kretszschmar MEE, Smits LJM, et al. Kosten-effectiviteit van algemene vaccinatie tegen hepatitis B (Interimrapportage). Bilthoven: RIVM 2000.

16. Glanz K, Lewis FM, Rimer BK (ed.). Health ehavior and health education: theory, research and practice. 2 ed. San Francisco: Jossey-Bass Publishers 1997.

17. Kok GJ, Bartholomew LK, Parcel GS, et al. Intervention Mapping: een protocol voor het ontwikkelen van op theorie en onderzoek gebaseerde gezondheidsvoorlichting. Tijdschr Gezondheidswet 2000;78:135-41.

18. Hospers H. Homosexual men and the HIV epidemic. Understanding and changing risk behavior. Dissertatie. Maastricht: Universiteit Maastricht 1999.

19. Empelen P van. Aids prevention among Dutch drug users. An intervention mapping approach. Dissertatie. Maastricht: Universiteit Maastricht 2001.

20. Schaalma HP. Planned development and evaluation of school-based AIDS/STD education. Dissertatie. Maastricht: Universiteit Maastricht 1995.

21. Schaalma H. Toepassen van Intervention Mapping: illustratie aan de hand van een lesprogramma voor Aidspreventie. Tijdschr Gezondheidswet 2000;78:395-400.

22. Lugoboni F, Migliozzi S, Schiesari F, et al. Immunoresponse to hepatitis B vaccination and adherence campaign among injecting drug users. Vaccine 1997;15:1014-16. 


\section{CHAPTER 5}

23. Rotily M, Vernay-Vaisse C, Bourlière $\mathrm{M}$, et al. HBV and HIV screening, and hepatitis B immunization programme in the prison of Marseille, France. Int J STD and AIDS 1997;8:753-9.

24. Mezzalani P, Venturini L, Turrina G, et al. High compliance with a hepatitis B-virus vaccination program among intravenous drug users. J Infect Dis 1991;163:923-4.

25. Borg L, Khuri E, Wells A, et al. Methadone-maintained former heroin addicts, including those who are anti-HIV-1 seropositive, comply with and respond to hepatitis B vaccination. Addiction 1999;94:489-93.

26. Purchase D, Mottram K, Miron C, et al. Hepatitis B vaccination for injection drug users, Piercy County. CDC MMWR Weekly 2001;50:388-90.

27. Sellors J, Zimic-Vincetic M, Howard M, et al. Lack of compliance with hepatitis B vaccination among Canadian STD clinic patients: candidates for an accelerated immunization schedule? Can J Public Health 1997;88:210-1.

28. Sellors J, Pickard L, Mahony JB, et al. Understanding and enhancing compliance with the second dose of hepatitis B vaccine: a cohort analysis and a randomized controlled trial. Can Med Ass 1997;157:143-8.

29. Dal-Ré R, González A, Ramirez V, et al. Compliance with immunization against hepatitis B. A pragmatic study in sexually transmitted disease clinics. Vaccine 1995;13:163-7.

30. Asboe D, Rice P, Ruiter A de, et al. Hepatitis B vaccination schedules in genitourinary medicine clinics. Genitourin Med 1996;72:210-2.

31. Bhatti N, Gilson RJC, Beecham M, et al. Failure to deliver hepatitis B vaccine: confessions from a genitourinary medicine clinic. BMJ 1991;303:97-101.

32. Dufour A, Remis RS, Alary M, et al. Factors associated with hepatitis B vaccination among men having sexual relations with men in Montreal, Quebec, Canada. Sex Transm Dis 1999;26:317-24.

33. Savage RB, Hussey MJ, Hurie MB. A successful approach to immunizing men who have sex with men against hepatitis B. Public Health Nursing 2000;17:202-6.

34. Weinstock HS, Bolan G, Moran JS, et al. Routine hepatitis B vaccination for sexually transmitted diseases. Am J Public Health 1995;85:846-9.

35. Yuan L, Robinson G. Hepatitis B vaccination and screening for markers at a sexually transmitted disease clinic for men. Can J Public Health 1994;85:338-41.

36. Anderson B, Bodsworth NJ, Rohrsheim RA, et al. Hepatitis B virus infection and vaccination status of high risk people in Sydney: 1982 and 1991. Med J Austr 1994;161:368-71.

37. Gilson RJC, Ruiter A de, Waite J, et al. Hepatitis B virus infection in patients attending a genitourinary medicine clinic: risk factors and vaccine coverage. Sex Transm Inf 1998;74:110-15.

38. Hoeven-Fritscher S, Kopp W. Hepatitis B bei personen mit hohem risiko für sexually transmitted diseases (STD). Screening und impfkampagne: akzeptanz und ergebnisse. Gesundheitswesen 1994;56:663-6.

39. Rowe TK, Douglass CW. Compliance with a voluntary hepatitis B vaccination program. J Dental Educ 1998;52:118-20.

40. Herold AH, Roetzheim RG, Pamies RJ, et al. Matriculants compliance with a required preventive health program. Acad Med 1992;67:403-5.

41. Havel RD, Wright MP. Automated interviewing for hepatitis B risk assessment and vaccination referral. Am J Prev Med 1997;13:392-5.

42. Marron RL, Lanphear BP, Kouides R, et al. Efficacy of informational letters on Hepatitis B immunization rates in university students. J Am Coll Health 1998;47:123-7.

43. Ganguly R, Marty PhJ, Herold AH, et al. Hepatitis B immunization in a university student population. J Am Coll Health 1998;46:181-3. 
44. Lawrence MH, Goldstein MA. Hepatitis B immunization in adolescents. J Adol Health 1995;17:234-43.

45. Cassidy WM, Mahoney FJ. A hepatitis B vaccination program targeting adolescents. J Adol Health 1995;17:244-7.

46. Rosenthal SL, Kottenhahn RH, Biro FB, et al. Hepatitis B vaccine acceptance among adolescents and their parents. J Adol Health 1995;17:248-54.

47. Middleman AB, Robertson LM, Young C, et al. Predictors of time to completion of the Hepatitis B vaccination series among adolescents. J Adol Health 1999;25:323-7.

48. Wilkinson SE, Morath M, Bennett DL, et al. Accelerated schedule of Hepatitis B vaccination in high-risk youth. J Pediatric Child Health 1996;32:60-2.

49. Moore-Caldwell SY, Werner MJ, Powell L, et al. Hepatitis B vaccination in adolescents: knowledge, perceived risk and compliance. J Adol Health 1997;20:294-9.

50. Dobson S, Scheifele D, Bell A. Assessment of a universal school-based hepatitis B vaccination program. JAMA 1995;274:1209-1213. 


\section{CHAPTER 5}

\section{CASE 4. OVERVIEW OF APPLIED PRACTICAL STRATEGIES}

\section{The administrative level}

Prevention of infectious diseases is a regular task under the Dutch Public Health Act (WCPV), for which the stakeholders at the local administrative level are responsible. In this case, however, the national government initiated and facilitated new policy to be implemented at the local level. A message was sent to the local authorities and the GGD Boards to inform these partners about the forthcoming policy adjustments and to invite local partners to participate by submitting proposals. Health promoters responsible for infectious diseases convinced the managing director of the GGD for Southern Limburg to propose this region as a pilot region in the national campaign, to improve the quality of hepatitis B vaccination for high-risk groups. Involvement of local government in this decision-making process was not considered necessary, because national policy overrules local policy. Besides, quality improvement is the responsibility of the GGD rather than the local authorities. Essential resources to participate in the campaign were allocated by the national government without local consequences in the short term. The local authorities were merely informed, as it was realized that future resources might become a negotiation issue between the GGD and the local government. In short, none of the practical strategies was deemed necessary at the local administrative level [advocacy -], [coalition building -], [sidestepping the formal system -], [agenda setting - , [resource negotiations - ].

\section{The institutional level}

The GGD management initiated organizational development by diagnosing the hepatitis B problem in the region, assessing the desired public health outcome and the willingness among agencies to take action. The diagnosis centred on the collaboration process, and involved the practice and research domains. Representatives of both of these domains thought it was not opportune to involve the local policy domain, as they had no task in the implementation [diagnosis +]. An action plan was prepared which assigned tasks to GGD professionals, consisting of participation in local and national working groups, a research proposal, programme development on collaboration, coordination of outreach activities and local monitoring [activities and interventions +]. Commitment was implicitly assumed, as all partners felt the urgency of combating infectious disease. No specific actions were undertaken to develop commitment strategies [commitment -]. An intersectoral collaboration structure was created, consisting of all relevant partners, such as research institute, addiction care services, 
STD clinics and prevention services, general practitioners, gay organizations, sex clubs, prisons and remand centres. Institutional managers appointed the local project leaders, who ensured that information was exchanged between the local and national working groups. The intersectoral management structure was formalized between the core partners as part of the grant conditions by contractual agreements between GGD, Maastricht University Hospital and Maastricht University [intersectoral management structure +]. Peripheral partners were contacted on an ad hoc basis by the local project leaders ${ }^{300}$.

Communication activities mainly focused on promoting awareness of the availability of HBV vaccination, both among high-risk groups and among intermediate organizations, for example general practitioners, mental health care service and gay organizations. Communication activities relating to the collaboration process as such were a consistent part of the regular local meetings. The communication structure was embedded in the intersectoral management structure [communication structure + ]. Institutional managers approved the rather comprehensive evaluation proposal that was drawn up by the local project leaders in collaboration with the core partners. The evaluation procedure included process-oriented aspects, such as the feasibility of collaboration, the frequency of joint meetings, costs and benefits, etc. [evaluation +]. The grant clearly distinguished between expenditures for staff, vaccines, communication materials, research, capacity building and miscellaneous [resources + ].

Practical strategies to improve the working climate were not explicitly considered during the pilot stage. Managers and staff who were involved in the project could be regarded as innovators themselves, supporting organizational development to collaborate and creating an atmosphere of enthusiasm [mission statement -]. During the dissemination stage, a learning climate at the local level was supported by national newsletters with up-to-date information on the campaign, using interviews and announcements about new materials or instructions [interviews with role models +$]^{303}$. Aspects of the working culture were addressed by arranging internships within the practice domain of the GGD and addiction care service, allowing interns to experience the working culture in these services, so they could advise on effective implementation. Maastricht University provided an intern who could assist the practitioners with the literature search that was to be part of the campaign preparations. In fact, however, this internship did not contribute to a link between the cultures of the domains involved, as the interns worked within their own domain [internships -]. Access to the working culture in other domains was enhanced by exchange between national and local institutions, i.e., between LCI, GGD, RIVM, Utrecht University and Maastricht University, and later on between the Netherlands Institute of Mental Health and Addiction (Trimbos-instituut), the Institute for Addiction Research of the Rotterdam Erasmus University and regional addiction care services. Institutional managers promoted mutual service rendering 


\section{CHAPTER 5}

(e.g., by providing leaflets, flyers, handouts, educational materials, newsletters), access to tools (e.g., electronic registration system, data files), negotiations on vaccine prices with the pharmaceutical industry, discussion of research designs, exchange of research findings [rendering services, access to tools +]. Formal and informal consultancy developed in a natural way through personal acquaintances [formal and informal consultations + ].

Capacity building at the local level was carried out by assessing staffing requirements and competence, a professional development policy, and feedback to staff [diagnosis and feedback +]. Training sessions were organized, which supported behavioural capability and self-efficacy as regards outreach interventions and collaboration with partners. Performance indicators were related to the number of vaccinated persons, targets for which could only be met by collaborating with partners [performance indicators and professional development + ]. No extra staff was recruited or retained [recruiting and retaining staff -].

A national kick-off conference was held after the pilot project had ended, to disseminate the experience acquired and to build capacity beyond the professionals who had taken part in the pilot study. Another conference was organized after the dissemination stage, to assess the benefits of collaboration between the policy, practice and research domains and to promote mutual capacity exchange [kick-off conference +]. The local project leaders frequently contacted all local partners involved and informed the local authorities [sidestepping the formal system + ].

\section{The individual level}

The local project leaders initiated consultations with the staff assigned to the project by the various agencies to assess the starting position in terms of staffing requirements and the actual competencies available, in terms of time, capacity, skills and tools [diagnosis and feedback +]. An action plan, regular feedback between multidisciplinary professionals of research institutes, the GGD, the addiction care services, the STD clinic and the penitentiary institutes contributed to a positive attitude, enthusiasm and positive norms and outcome expectations [group discussion in multidisciplinary groups +]. The most commonly applied practical strategy for practitioners and researchers was learning by doing, achieved through task assignment, formats, materials and feedback [learning by doing +], [detailed action plan, formats, materials + ]. No other practical strategies were applied to promote collaboration between policy makers, practitioners and researchers [feedback by peer networking -], [lectures and discussion -], [master classes with skills training -]. National and local working group

members stimulated and coached each other to publish in scientific as well as professional journals to disseminate the knowledge to a wider audience of researchers and practitioners ${ }^{304-306}$. For the same reason, project leaders contrib- 
uted to conferences and workshops (5 times). Policy makers were not asked to become involved in this process [in-service coaching to publish beyond one's own niche +-$]$.

\section{Comments}

So far, the hepatitis B vaccination campaign can be considered a success. In retrospect, the elements that have contributed to this success are the national policy strategy, which meant that local partners (especially GGDs) could avoid time-consuming practical strategies at the administrative level, such as lobbying, bargaining and advocacy to get the issue on the local policy agenda. The national goal was clearly formulated, namely to increase the number of hepatitis B protected high-risk persons. There were no restrictions on the methods to achieve this goal, and tailoring to local conditions was promoted. Leadership was perceived as less important, in view of the national policy directive, which overuled the local administration.

At the institutional level, the practical strategies focused on the collaboration between practitioner agencies and research institutes, both nationally and locally. The institutional managers from the local policy domain were not closely involved, and were merely informed during regular meetings between the GGD management and the head of the municipal Department of Education, Culture, Welfare and Health.

The innovation stage was facilitated by the Ministry of Health, Welfare and Sport by means of long-term resource allocation and continued guidance. During the innovation stage, organizational development could take place, a culture of reciprocity and collaboration was achieved, and capacity was built, all serving to provide individuals with the tools to appreciate the niche differences and to bridge the gaps between them. The reciprocal relations between national and local services clearly resulted in synergy. Training and peer networking for researchers and practitioners were initiated by the partners at the national level and coordinated by the national association of GGDs. Expensive materials were prepared at the national level and applied at the local level. Local practice and research experiences were translated to the national level and were used in policy evaluation. Steps 1 to 4 of the research cycle were first prepared by means of a literature search before the HBV campaign was developed (see the enclosed article). Next, the practice work cycle was used to assess the feasibility of the HBV campaign in local practice. Subsequently, the research and practice cycles started simultaneously to study the effect of the campaign, and to monitor its dissemination. Conditions for dissemination and effectiveness identified by research were fed into the policy work cycle in order to adjust national policy. Work cycles repeatedly interconnected, creating a helix, to achieve incremental improvements in HBV research, practice and policy. The responsibility 


\section{CHAPTER 5}

for continuation after the pilot stage was formalized by making HBV part of the local public health policy. No conflicting political views appeared when HBV was included in the Diversified Products Provision Model used by the Southern Limburg municipalities. In this case it was relatively easy to adjust the Model because no financial requests were made to local government. The ministry agreed to continue to provide the vaccine free of charge. The gaps between the policy, practice and research work cycles were successfully bridged, albeit that only the national policy domain was involved, while active participation by the local policy domain was negligible. Collaboration between the practice, research and policy domains resulted in evidence-based public health interventions, and empirical data showed the effectiveness of the campaign ${ }^{307,308}$. Many outreach strategies were developed, which were applicable in practice, and beneficial to the target population in facilitating the vaccination service.

In all, the national policy will take ten years to complete (1998 to 2008). From 2007 onwards, the hepatitis B vaccination campaign will be part of the GGD's regular tasks, financially supported by the Ministry at least until 2008. We may conclude that a nationally developed policy programme may have a positive impact at the local level, provided that the programme can be tailored to the local situation, and is financially supported. However, if these conditions are not met, such a programme may be counterproductive. This case clearly illustrates the benefits of such a national policy programme, which avoids timeconsuming agenda-setting procedures and high demands on leadership qualities, and ensures reciprocal relations between local and national expertise. 
MULTIPLE CASE STUDY

\subsection{CASE 5. INTRODUCTION}

\section{The tailored approach of the 'Schoolbeat' programme: new concepts for health promotion in schools in the Netherlands}

A healthy start in life is a crucial factor in building a healthy feature. Policy makers and various organizations involved in public health therefore frequently stress the importance of good health promotion and preventive care for young people. And yet, in Dutch schools - the one place where all young people gather - health promotion and preventive care are fragmented and supplydriven, and rarely address directly the needs of a specific school population. In general, collaboration is still limited among the organizations that support schools, resulting in inefficiency and an insufficient critical mass. In 2002, the GGD together with Maastricht University, the Netherlands Institute for Health Promotion and Disease Prevention (NIGZ) and five regional partners started the development of a collaborative approach to tailored school health promotion, in a programme called Schoolbeat (schoolSlag in Dutch). The partners came from the areas of welfare, addiction care, public health, mental health care, social care and school care. The aim was to reduce risk behaviour in youth (4-19 years) in the Maastricht region over a period of ten years. Risk behaviour and bad health are known to hinder educational achievement. Therefore, reducing risk behaviour and enhancing health are crucial. The project midterm objectivese focus on establishing a long term collaboration between the five health promoting agencies to implement school activities that are tailored to the needs of teachers, parents, pupils and schools ${ }^{309}$.

Attaining a higher level of evidence-based school health promotion requires an intensified contribution from the research domain, as there have so far been few evidence-based programmes. Getting schools to participate in the Schoolbeat strategy required the development of a local policy instrument in which the municipal Departments of Education and Public Health integrated their goals to stimulate participation in school health promotion programmes. The Schoolbeat programme would only be feasible if the policy, practice and research domains collaborate.

A 10-year time horizon was set to allow effects on the reduction of risk behaviour among young people to be measured. Based on research about the processes of adoption, implementation, maintenance and institutionalization, we concluded that the institutionalization (or routinization) of an innovative operational method by schools and their collaborating partners is an incremental process, taking about ten years to develop successfully. A provisional evaluation shows that the Schoolbeat strategy appears to be successful, although further implementation, research and policy commitment are necessary. 


\section{CHAPTER 5}

The article included below describes the preparation of the Schoolbeat strategy, its programme content and how it should be organized. The article forms a small part of the total programme implementation. Phase II of the Schoolbeat programme will start in 2007 , focusing particularly on the dissemination of the innovation throughout the province of Limburg and pilot regions elsewhere in the Netherlands. In addition, specific topics relating to this approach will be addressed by a so called 'Academic Collaborative Centre for Public Health' (Academische Werkplaats). The Academic Collaborative Centre for Public Health was founded in 2006 and aims to integrate practice and research to strengthen the local knowledge infrastructure and local policy in order to develop and implement evidence-based public health strategies. 
Published in: Stephen Clift \& Bjarne Brunn Jensen (eds). The Health Promoting School: International advances in theory, evaluation and practice. Copenhagen, The Danish University of Education 2005: 87-106.

The Tailored Schoolbeat Approach: New Concepts for Health Promotion in Schools

Mariken Leurs, MPH, GGD Zuid Limburg

Maria Jansen, MSc, GGD Zuid Limburg

Herman Schaalma, Assistant Professor at the Department of Health Education and Health Promotion, Maastricht University

Ingrid Mur-Veeman, Assistant Professor at the Department of Health Organization Policy and Economics, Maastricht University

Nanne de Vries, Professor at the Department of Health Education and Health

Promotion, Maastricht University 


\section{CHAPTER 5}

\section{INTRODUCTION}

The first developments in school health promotion in many European and English-speaking countries date from early $20^{\text {th }}$ century. Developments in the area of school-wide health promotion are now widespread, especially in the English-speaking countries ${ }^{1-4}$. However, the inclusion of comprehensive health promotion in school policies remains a challenge as education and not health is the core business of schools ${ }^{5}$.

As a member of the European Network for Health Promoting Schools since the mid 1990s, the Netherlands developed a national action plan on school health promotion over a number of years. This plan focuses on the three 'historical' domains: classroom health instruction, school health services and a healthy school environment ${ }^{6}$. Results so far are limited: school health promotion and preventive youth care activities in the Netherlands are fragmented, supply-driven, primarily focused on individual pupil care and addressing the specific needs of a school and its population rarely directly ${ }^{7-9}$. As is the case in other countries, few health promoting school (HPS) interventions have been evaluated and even fewer have proven to be effective ${ }^{10-12}$. This is changing with recent increases in the number and breadth of evidence-based schoolbased prevention programs and effectiveness research becoming a central focus of research activity in this area ${ }^{13}$. Hence, it came as no surprise that the effectiveness of specific HPS-interventions was marked as the number one priority of the international HPS-research agenda at the $18^{\text {th }}$ World Conference on Health Promotion and Health Education in Melbourne in $2004^{14}$.

Recently, a bottom-up approach for school health promotion was initiated in the Netherlands. This article describes this bottom-up approach, dubbed 'schoolBeat', 'schoolSlag' in Dutch. The approach has a strong focus on the establishment and monitoring of sustainable intersectoral collaborative support for comprehensive school health promotion. This is one of the key strategies advocated recently by Deschesnes and colleagues to enhance broad implementation of comprehensive approaches to school health ${ }^{15}$. Additionally, schoolBeat aims to develop and introduce a specific tailored approach to comprehensive school health promotion, involving - in first instance - school staff, pupils and parents. The coalition partners take responsibility for disseminating congruent messages into the surrounding community. Hence, a multifaceted approach to multiple determinants will be created. As this is a complex HPS initiative, its evaluation will be challenging ${ }^{16}$. This article includes a description of a new model for evaluating the collaborative aspects of our approach - the DISC-model - as part of this evaluation process. 


\section{THE SCHOOLBEAT APPROACH}

The development of schoolBeat commenced in 2001 when five regional health promoting agencies joined forces in the south of the Netherlands. The five keyplayers came from the areas of addiction, mental health, public health, youth care and social welfare. With the recruitment of a project manager and researcher, financed by a national four-year grant, the project advanced in Spring 2002.

In ten years, schoolBeat aims to reduce risk behaviours among youth (4-19 years) in the Maastricht region. The project's midterm objectives (2005) focus on establishing sustainable collaboration among schools, health promoting agencies and local authorities. The number and quality of tailored health promotion activities should also be increased in this period. In order to pursue these objectives a systematic plan of coordinated support for tailored school health promotion policy was developed. The plan is based on the principles of intervention mapping ${ }^{17}$ and tailored to the possibilities and pitfalls of the educational system and the health system in the Netherlands. Forms of action research were used in combination with literature reviews and expert consultations ${ }^{18-20}$. However, programs cannot be developed based on expertise and authority alone. It requires full participation of all stakeholders ${ }^{21}$. Hence, the development of schoolBeat includes participation of stakeholders from the health, welfare and education sectors. This is a common type of collaboration in school health promotion ${ }^{4,5,22,23}$.

As part of the process, new concepts were introduced in the area of (1) participation of the entire school population in HPS, (2) quality assessment of HPS-interventions, (3) workload sharing among regional support organizations, (4) linking school health promotion to individual pupil care and (5) diagnoses of the development of sustainable collaboration using the newly developed DISC-model ${ }^{24-27}$. An in-depth description of each specific innovation is beyond the scope of this general introductory article.

SchoolBeat-study I, accompanying the development-phase of schoolBeat, spans the first four years of development, preliminary implementation and adjustments of the approach, primarily using action research.

Before describing the steps of the schoolBeat approach, including the introduction of new concepts where appropriate, the main planning-principles of 'Intervention Mapping' will be outlined. These principles are widely applicable to health promoting school developments. 


\section{CHAPTER 5}

\section{INTERVENTION MAPPING PRINCIPLES}

A sound Intervention Mapping process provides program planners "with a framework for effective decision making at each step in intervention planning, implementation and evaluation"17, with interventions being defined as a "planned combination of theoretical methods delivered through a series of strategies organized into a program". The specific focus of Intervention Mapping is the evidence- and theory-based development of health education and promotion using a socio-ecological approach to health. This is in line with the holistic approaches to school health promotion, popular since the mideighties 28,29 . Basically, both paradigms focus on the wide picture of interrelationships among individuals with their personal characteristics and their environments. Intervention Mapping identifies the most effective points and accompanying strategies for interventions in this complex picture and eliminates the use of an ineffective trial-and-error approach. It is a comprehensive and pragmatic step-by-step approach to the development, implementation and evaluation of health education and promotion interventions. A form of needs assessment precedes the Intervention Mapping steps. Intervention Mapping starts with (1) a specification of evidence-based program objectives regarding behaviour and environmental conditions. This is followed by (2) the selection of intervention methods and strategies with a sound theoretical base and (3) program design, pre-test and production. Additionally, (4) adoption and implementation plans are developed integrally, with a focus on sustainability. This all should be supported by (5) an evaluation plan ${ }^{17}$. This evaluation is not only meant to judge the planned intervention on effectiveness, but also to facilitate understanding of all stakeholders ${ }^{30}$. Overall, Intervention Mapping is an iterative process. New insights gained along the way, will adjust choices made in previous or future steps resulting in an adjusted, more effective program. As a planning model, Intervention Mapping builds strongly on previous models by Green and colleagues ${ }^{31,32}$.

To engage successfully in Intervention Mapping, insights are required into the needs and capacities of the intended target group (individuals and communities) and into the current state-of-play in health education and promotion evidence and theories ${ }^{17}$. As far as school health promotion is concerned, it is important to take into account differing objectives of the health promotion agencies (i.e. health) and schools (i.e. education) prior to engaging in any intervention mapping process regarding HPS-interventions ${ }^{33}$. Or, as stated by Green and Kreuter 32, p. 392: "Experiences around the world have taught planners this lesson: failure to acknowledge and address the perceptions and feelings held by administrators, teachers and parents, however difficult those sentiments may be to quantify, can stop the best-designed, well-intended program dead in its tracks." Therefore, it is important to be aware of the existing evidence regard- 
ing the potential positive impact of school health promotion on school curricula and knowledge of pupils ${ }^{33,34}$.

The schoolBeat approach is based on the Intervention Mapping steps described. This will be illustrated by outlining the approach using the IM steps described in the next section.

\section{THE SCHOOLBEAT STEPS TOWARDS A HEALTHY SCHOOL}

The systematic schoolBeat approach includes coordinated support of schools during - what is defined from the perspective of schools - the 'schoolBeat steps towards a Healthy School'. This support takes in the form of account managers (mostly health promotion professionals) with advisory tasks on behalf of the collaboration. They are called 'schoolBeat advisors'. This concept implies workload sharing among the collaboration partners in attracting and supporting schools. It requires regular consultation between the schoolBeat advisors as well as educating the advisors regarding the schoolBeat approach and the fields of expertise of the different collaborating partners. This is done to pro-actively deal with possible difficulties due to a lack of understanding among the partners of how sectors work and function as suggested by the findings of school health promotion programs with a major partnership component ${ }^{33}$.

The first two steps in the schoolBeat approach are the prerequisites for the application of intervention mapping principles from step 3 onwards. Hence, the 'schoolBeat steps towards a Healthy School' come down to a specification of the intervention mapping principles to the school setting, extended with two 'preparation' steps. Regarding schoolBeat, it should be noted that the 'schoolBeat steps towards a Healthy School' focus on the school-based process, without taking full account of the back-office structure and activities of the collaborating partners supporting this process. The schoolBeat steps can be described as follows:

\section{Determining the health needs of the school}

The health needs of a school cannot be based on available epidemiological data regarding the health status of students alone ${ }^{17,35}$. In the Netherlands, and possibly elsewhere as well, there is a tendency among regional public health institutes to do just this, as this data are relatively easy available. However, it is important to also include data on the educational performances of students, registration of absence due to illness among students and staff sick leave, issues coming up in staff and parent meetings regarding school health policies and information on the current status of the school's organization, housing and activities with a possible impact on school health ${ }^{36}$. A schoolBeat advisor is available to assist schools in clarifying and interpreting these types of information. It is preferable 


\section{CHAPTER 5}

that at least one partner-organization has the capability, expertise and personnel to compile school health profiles for each school within the HPS-scheme. It is important to stress that this information is compiled with the school instead of for the school, as the most important source and data interpreter needs to be the school itself. It was found to be extremely important that the major stakeholders in a school recognize themselves in the data provided and that they be able to complete the picture with internal data sources and interpretations. By the major stakeholders we mean school administrators, prevention and care coordinators, teaching staff, students (especially in secondary schools) and parents. Involvement in this needs assessment process, which continues in the next step, by stakeholders is likely to increase awareness, create "ownership" of the program and build commitment ${ }^{35}$.

In many of our schools this step included the installation of a school health promotion team with representatives of the major target groups in schools. This could be a new team or an extension of an existing school team, for example a working group on the prevention of substance abuse in school. This school-based health promotion team (some schools refer to this team as the 'schoolBeat team') is related to the school care team in order to maximize opportunities regarding an integrated approach to school health, based on an extended comprehensive view on shared care $^{37}$. It links health promotion to other school-based interventions. According to St Leger and Nutbeam ${ }^{5}$ and Greenberg ${ }^{13}$, this link is one of the priorities in school health promotion that needs to be pursued in the coming decade.

\section{Setting health promotion priorities}

Based on the information described in step one, a school can determine its school-health priorities, including health promotion. Schools are advised to limit their priorities to around six or eight items and to have them recognized by the school board.

As described in step one, participation of students, staff (educational as well as support staff) and parents can be achieved by organizing a school health team. In practice, this means a school care team and a school health promotion team as two separate but linked entities. As the introduction of specific teams limits the level of active participation to a restricted number of stakeholders, other participation strategies for the selection of health promotion priorities are welcome.

While working with schools, the knowledge of school-based stakeholders regarding the activities their own school undertakes in the area of health promotion and the information upon which choices are based were found to be limited. This was supported by previous findings of Marshall and colleagues 3 . Hence, we were not surprised about the limited support for school health promotion. To raise support for school health promotion and increase general 
knowledge on the possible choices and current actions in school health promotion, a healthy school priority-workshop was adopted. Originally, this workshop was developed for staff and parents of primary schools ${ }^{38}$. To be applicable to students, parents and staff in secondary education the workshop needed adjustments. Based on expert consultation, explorative research among the three target groups (i.e., students, staff and parents) and pilots in different settings (i.e., classroom setting, parent evenings, mixed meetings of staff and parents and mixed meetings of students, staff and parents) an adjusted workshop was developed specifically for secondary education schools ${ }^{24}$. This adjusted workshop differentiates the priorities based on the components of the Comprehensive School Health Program ${ }^{1,39}$. After conducting the schoolBeat workshop, stakeholders reported an increase in internal support for school healthpromotion activities and an increase in knowledge regarding school health promotion among workshop participants. They perceived the results of the workshop as being relevant for tailoring school health promotion to the demands of their own school population. Joint actions have not yet been reported. However, one should take into account that these findings are preliminary and might be biased, as they are not based on rigorous research.

\section{Assessing the important and changeable determinants}

Even though the Intervention Mapping protocol includes the setting of health promotion priorities and the selection of important and changeable determinants in step one $e^{17}$, we separated these two aspects in distinct steps. This is done to emphasize the importance of a clear analysis of the situation instead of implementing projects that seem to address the health promotion priorities set too quickly, without further analysing whether these projects focus on the most important and changeable determinants of the priorities set. This step is very much a task for the experts of the support organizations in their role as school health advisors. For example, when a school sets a priority regarding the promotion of safe sex among students, the advisor looks for the different determinants of safe sex among adolescents. This may be knowledge regarding the risks of acquiring sexually transmitted diseases or getting pregnant. Other determinants are skills of students to acquire condoms and the availability of condoms in 'safe' places for students like school toilets. Based on this analysis, the advisor will look at the importance of the different determinants with regard to expected effects on the set priority. Additionally, the level of changeability of this determinant will be assessed in order to provide schools with realistic advice.

\section{Compiling the school health plan}

The fourth schoolBeat step corresponds with step two in Intervention Mapping: 'selecting theory-based intervention methods and practical strategies' and 


\section{CHAPTER 5}

compiling them into a whole-school plan. Evidence-based interventions are rare, so the choice for 'theory-based' is a logical one. However, little has yet been reported on the theoretical basis of most school health interventions. In this respect, the Dutch situation seems common worldwide. This led to the development of an instrument for assessing the quality of school health promotion interventions ${ }^{27}$. It is assumed that the use of a specific quality check based on quality criteria from the health promotion and education domains would improve overall quality of a comprehensive school health promotion plan in terms of the effectiveness and adaptability within the school of selected prevention programs. The schoolBeat quality-instrument is based on consultations of experts from both fields (health and education) and a review of other possibly relevant quality indicators ${ }^{40-43}$. Table 1 presents the nine criteria on the checklist. Each criterion is operationalized by a set of items, differing between two and ten items per criterion. Scoring is done per item on a three-point scale.

Table 1 Criteria of the SchoolBeat quality-checklist 1.0

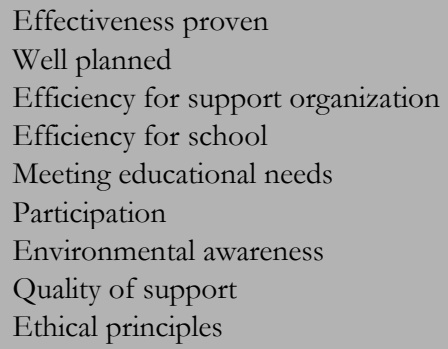

In order to structure the program and activity choices, the American Coordinated School Health Program ${ }^{39}$ was adapted to the Dutch situation. Proposals for changes were based on the health and education structure and priorities in the Netherlands and sanctioned by the managers of the collaborating schoolBeat partner-organizations. This process yielded a slightly adjusted 'Healthy School Model' 37 , as illustrated in figure 1. 


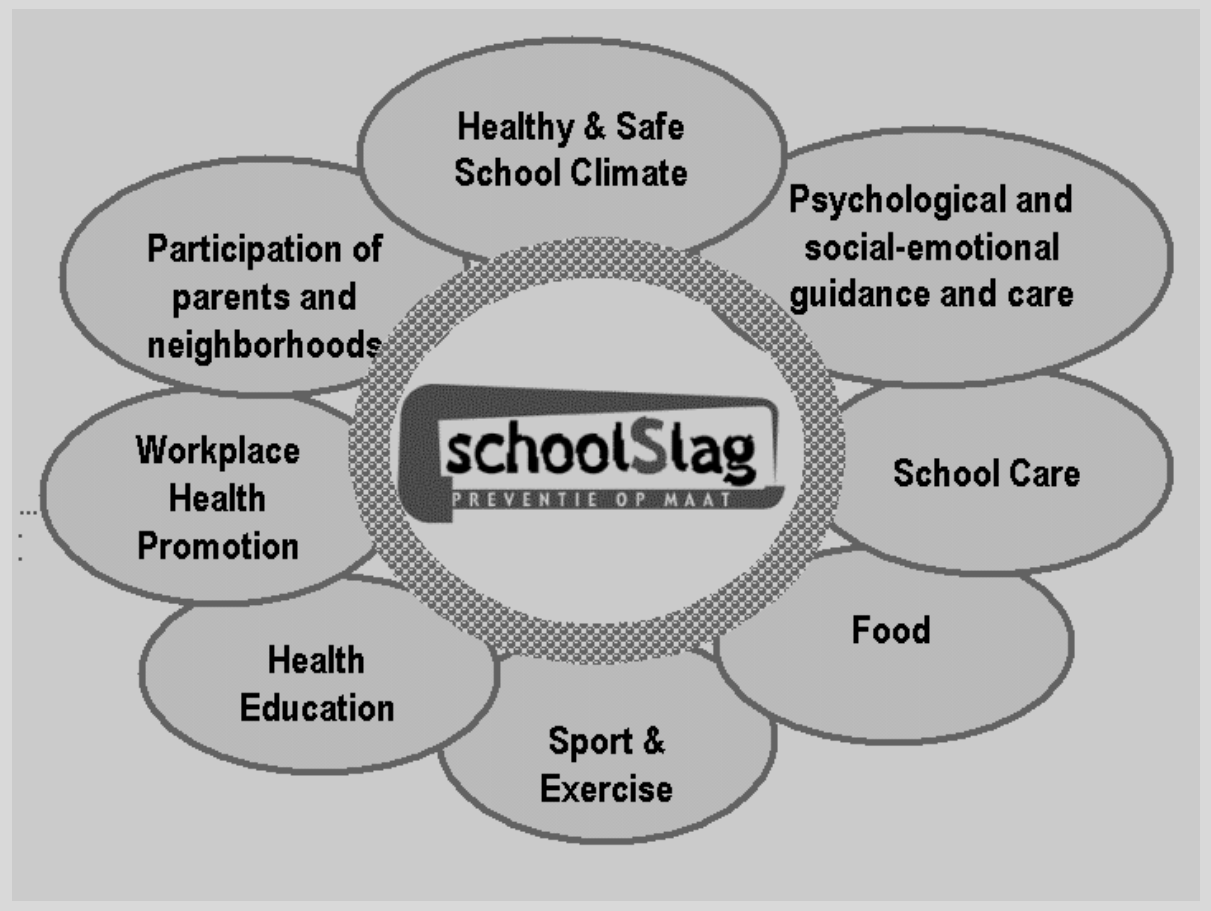

To assist schools, we use a matrix with the different target groups (i.e., students/classes, teachers, parents) on one axis and the selected health priorities on the other. Filling in the different cells, it became clear that secondary schools focus mainly on interventions targeting healthy student behaviour in the first three curriculum years. This was an eye-opener for schools, because they did not yet have a clear view of their overall input in school health. It became a challenge for the schools to fill in the cells for the other target groups. Schools decide themselves what to do. It appears that they have several relatively simple and often sound ideas on how to achieve progress in some of the areas. Support organizations come in with additional advice on effective approaches and solutions that suit the implementation possibilities of schools.

\section{Realizing the school health plan}

As with the other steps, the adoption, implementation and sustainability of the school health plan is the responsibility of the school itself. Health promoters may play a supportive role, where necessary and desired by the school. Schools have a long tradition in developing annual and long-term school plans. A school health plan should be very much an extension of this school plan. Where possible, it should be included in the school plan as an integral part of 


\section{CHAPTER 5}

school policy. Thus, linking school health promotion once again with other school-based activities as stressed by St Leger and Nutbeam5. This step contains a lot of useful information that health promotion agencies - regional and national - may be able to learn from schools. Their support ought to be adjusted accordingly. This may strengthen the expertise and skills already present in schools and fill existing 'gaps' that appear.

This fifth schoolBeat step coincides with the fourth Intervention Mapping planning phase (planning program adoption, implementation and sustainability). Special attention needs to be paid to the commitment of all stakeholders in the realization of the plan, not only in the planning phase but also in the implementation phase. Those involved in the planning phase must be informed about progress and possible outcomes. If possible, they should be able to experience certain aspects of the entire school health-promotion plan themselves.

\section{School-based evaluation}

Evaluation is an element of the schoolBeat methodology which needs to be considered right from the very start as evaluation not only deals with the effects on health and behaviour, but also with the process of school health promotion. Specifically, in the first years of introducing and implementing a systematic tailored whole-school approach, it is the process evaluation, which needs attention. When taking an action-research approach, the newly gained insights may be used directly to adjust processes, where needed. Anchors for effect evaluation in later years should not be forgotten. Attitudes, knowledge and satisfaction regarding the new approach, especially of school staff and administration, are important indicators to take into account. They are the main gateway to the wider school population: students, other teaching and support staff and parents. To limit the research burden on schools we ensured that instruments used for needs assessment purposes can be used for evaluation purposes as well. Support organizations with tasks in the area of epidemiology can assist schools in this area as well.

The two latter schoolBeat steps have not yet been described in detail as we do not yet have the necessary field-experience with the implementation of these steps. In future publications this will be addressed.

\section{EVALUATION}

The evaluation of the schoolBeat approach - the schoolBeat collaboration and its account managers and the school-based schoolBeat steps - focuses on the extent to which coordinated and tailored school health promotion is realized in the Netherlands in 2010 and the results it yields in terms of the levels of healthy behaviour and healthy schools. This includes research into the collaborative 
aspects of this comprehensive working procedure, which has much in common with the apparent increasing worldwide interest in productive partnerships ${ }^{13,18}$, 44,45 .

In order to monitor and evaluate the collaboration process and to be able to adjust procedures where required, we have developed a research model for 'DIagnosis of Sustainable Collaboration' (DISC) ${ }^{26}$. By doing so, we went beyond the more traditional evaluation models used in health promotion focussing primarily on the implementation and effects of single intervention programs. The DISC-model is based on the WIZ-model used for coordination and integration of health services and reviews into networking, collaboration and implementation in the area of health promotion ${ }^{46-49}$. The DISC-model focuses on the interaction between the project management and the perceptions, intentions and actions of the collaborating partners together (the project-support group), the project organization and factors in the wider context (figure 2). The DISC-model links the collaborative approach directly to the real-life context in which the approach develops, making it appropriate for case study designs ${ }^{50}$.

Figure 2 Diagnosis of Sustainable Collaboration (DISC) model

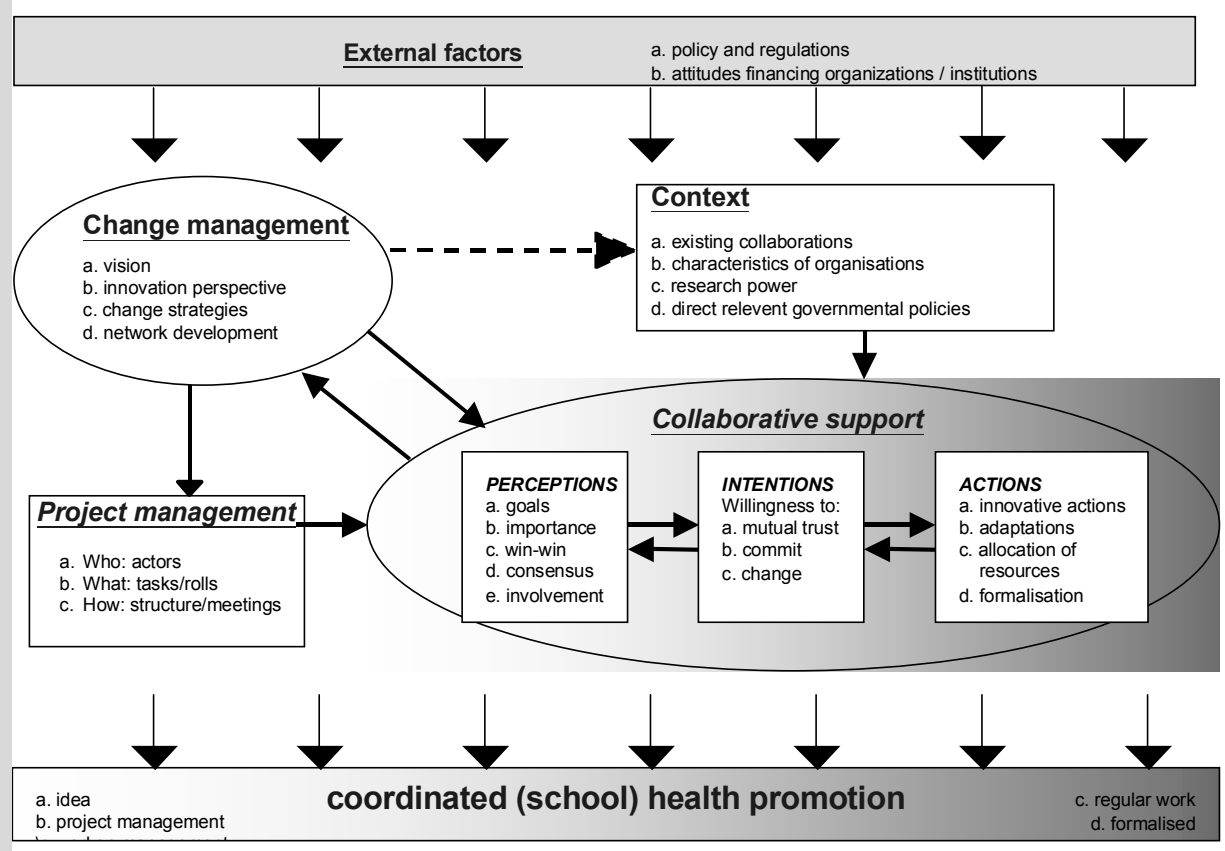




\section{CHAPTER 5}

Process evaluation of the schoolBeat approach using the DISC-model is done by means of a survey among stakeholders from the collaborating partners (schools, municipalities and health promotion organisations) followed by indepth interviews. The survey was piloted in a nearby region using the regional youth prevention network as a test case. Preliminary results indicate that especially municipalities and schools perceive schoolBeat as a new intervention, not differentiating it from interventions like substance abuse prevention programs for schools and bullying prevention plans. They do not seem to perceive schoolBeat as an advanced working procedure aimed at improving the match between interventions and the needs of a school. Additionally, local authorities fear the costs of schoolBeat following the development phase, which is financed by a national grant, as the coordinating costs are no longer covered. However, right from the outset of the schoolBeat development municipalities have made clear that the working method to be developed should not add costs to current investments in health promotion. Although the collaborating partners have developed the project with this in mind, municipalities do not seem convinced. The outcomes call for additional and more focused communication. Within the schoolBeat project-management structure, this will be a challenge for the schoolBeat communication group to address.

At the national policy level in the Netherlands there is a focus on investing in young people in order to reduce inequalities in health and to increase safety levels in society. It is a challenge for all health promotion professionals to profit from this - in DISC terms - 'external factor' on behalf of the health promoting schools.

The DISC-model only serves as a diagnostic tool. Actions to be taken to improve the diagnosed situation have to be decided on collaboratively. For example, "the Partnership Analysis Tool: for partners in health promotion" 51 may be used to support the decision process when progress is needed at the level of the initial health promotion partners. In this initial phase, it should be decided on within the project management structure. This evaluation and adaptation process ought to involve the key-stakeholders in meaningful ways. This fits the contemporary community-evaluation principles as formulated by Goodman ${ }^{52}$. In due time the project management structure it to be phased out, once the schoolBeat method has been adopted as part of the regular working procedure of health promoting agencies and schools. It should be replaced by a sustainable network structure or integrated in an already existing collaboration. These issues require communication efforts of the collaborating partners, supported by the schoolBeat coordinator. Studies on applications of this model should indicate the added value of the model as a diagnostic instrument for health-promotion collaborations, if present. Hence, it is also possible that the model itself will need to be adjusted and will 'change colour' as well. 


\section{CONCLUDING REMARKS}

The schoolBeat approach is made up of six - relatively easy to apply - steps as part of a coordinated support of tailored school health promotion. Field experience with these steps is still limited. From other studies, it is widely accepted that general community programs may take years to produce results ${ }^{52}$. Hence, little can be said about its proven effectiveness as yet.

During the initial development of the schoolBeat approach some shortcomings were identified, which were addressed as well. As some of the introduced new concepts in school health promotion deal with one or more of the priority areas for enhancing the effectiveness of school health promotion, it seems worthwhile to take a long-term perspective with this approach. In the meantime, some of these new concepts have been lifted from the regional level to the national level to facilitate long-term regional implementation. For example, application of the schoolBeat quality checklist to nationally available school-based health promotion programs does not fit fully with the set tasks of regional health promotion agencies. On the other hand, application of the checklist and making assessment results publicly available does fit with tasks set by the National Government for National Health Promotion Institutes to support regional and local health promotion.

Based on theoretical planning, formative research and preliminary fieldwork, we have high expectations of the added value of the 'schoolBeat qualitychecklist' and the 'DISC-model' in the field of school health promotion. The use of the quality checklist and the diagnostic model in other countries and cultures is welcomed in order to gain a wide spectrum of field experiences and insights into possible points for improvement.

\section{Acknowledgements}

The authors wish to thank the schoolBeat coalition partners (schools, municipalities, health promotion and welfare organizations: Bureau Youth Care Limburg, RIAGG Maastricht, Mondriaan Zorggroep, Trajekt, Maastricht Public Health Institute, NIGZ and Maastricht University) for their constructive advice and support in developing and realizing the schoolBeat approach.

The schoolBeat study is supported by a grant from ZonMW Healthy Living (project 4010.003) and from the OGZ Foundation (project 342).

\section{References}

1. Kolbe, L.J., Increasing the impact of school health promotion programs: emerging research perspectives. Health Education, 1986. 17: p. 47-52.

2. Rogers, E., et al., Developing the 'health promoting school' - a national survey of healthy school awards. Public Health, 1998. 112: p. 37-40. 


\section{CHAPTER 5}

3. Marshall, B.J., et al., School-based health promotion across Australia. Journal of School Health, 2000. 6: p. 251-252.

4. Goffin, A., et al. Successful inter-agency collaboration: health agencies working together to better promote health in schools. In: World Conference on Health Promotion and Health Education. 2004. Melbourne.

5. St Leger, L. and D. Nutbeam, A model for mapping linkages between health and education agencies to improve school health. Journal of School Health, 2000. 70(2): p. 45-50.

6. Buijs, G., et al., Landelijk actieprogramma schoolgezondheidsbeleid. National action plan school health promotion. 2002, NIGZ: Woerden.

7. Van Veen, D., C. Day, and G. Walraven, Multi-Service Schools. 1998, Leuven/Apeldoorn: Garant Publishers.

8. Pijpers, F.I.M., Schoolgezondheidsbeleid in het basisonderwijs. School health policy in primary education. 1999, Leiden University: Leiden.

9. Paulussen, T., Voeren scholen een gezondheidsbeleid? Do schools execute a health policy?, in Tijd voor gezond gedrag - bevordering van gezond gedrag bij specifieke groepen. Time for healthy behavior - promotion of healthy behavior among specific groups, J. Jansen, A.J. Schuit, and F. Van der Lucht, Editors. 2002, RIVM/Bohn Stafleu Van Loghum: Bilthoven. p. $134-141$.

10. Schaalma, H.P., et al., Planned development and evaluation of AIDS/STD education for secondary school students in The Netherlands: short-term effects. Health Education Quarterly, November;23, 1996. november 23(4): p. 469-87.

11. Cuijpers, P., et al., The effects of drug abuse prevention at school: "The Healthy School and Drugs" project. Addiction, 2002. 97: p. 67-73.

12. Van Lier, P., et al., Preventing disruptive behavior in elementary schoolchildren: I impact of a universal classroom-based intervention, in Preventing disruptive behavior in early elementary schoolchildren (dissertation), P.A.C. Van Lier, ed. 2002, Erasmus University: Rotterdam.

13. Greenberg, M.T., Current and future challenges in school-based prevention: the researcher perspective. Prevention Science, 2004. 5: p. 5-13.

14. Leurs, M.T.W., Gezondheidsbevordering en preventie op school - verslag van de World Conference on Health Promotion \& Health Education School health promotion - report of the World conference on Health Promotion \& Health Education. Tijdschrift voor Gezondheidswetenschappen, 2004. 82(7): p. 467-468.

15. Deschesnes, M., C. Martin, and A. Jomphe Hill, Comprehensive approaches to school health promotion: how to achieve broader implementation? Health Promotion International, 2003. 18(4): p. 387-396.

16. Stewart-Brown, S., Evaluating health promotion in schools: reflections. In: Rootman, I., Goodstadt, M., Hyndman, B., McQueen, D.V., Potvin, L., Springett, J. and Ziglio, E. Evaluation in health promotion - principles and perspectives. World Health Organization, Denmark, 2001: p. 272-284.

17. Bartholomew, L.K., et al., Intervention mapping: designing theory- and evidence based health promotion programs. 2001, Mountain View, CA: Mayfield.

18. Peters, L.W.H., De Netwerkbenadering - review. The network approach - review. 2001, Woerden: NIGZ - Centre for Review and Implementation.

19. Leurs, M.T.W., M. Jansen, and M. Ruiter, Coordinated School Health Program: het 'Gezonde School Model' van de toekomst? CSHP: the Healthy School Model of the future? Tijdschrift voor Gezondheidswetenschappen, 2002. 80(7): p. 471-473. 
20. Peters, L.W.H. and F.E.M. Keijsers, Kwaliteitscriteria voor gezondheidsbevorderende interventies in en rondom scholen: review en checklistontwikkeling. Quality criteria for school health promotion interventions: review and checklist development. 2002, Woerden: NIGZ - Centre for Review and Implementation.

21. Wallerstein, N., M. Polascek, and K. Multrud, Participatory evaluation model for coalitions: the development of system indicators. Health Promotion Practice, 2002. 3(3): p. 351-373.

22. Jones, J., et al., Health and Education Working Together: The New South Wales SchoolLink Initiative. International Journal of Mental Health Promotion, 2002. 4(4): p. 36-43.

23. Lee, A., C. Tsang, and S.H. Lee, A comprehensive "healthy schools programme" to promote school health: the Hong Kong experience in joining the efforts of health and education sectors. Journal of Epidemiology and Community Health, 2003. 57: p. 174-177.

24. Buijs, G., M. Ausems, and M. Leurs, De schoolSlag-prioriteitenworkshop Voortgezet Onderwijs. The schoolBeat priority workshop Secundary Education. 2004, NIGZ: Woerden.

25. Leurs, M.T.W., et al., Development of a collaborative model to improve school health promotion in the Netherlands. Health Promotion International, 2005. 20(3): p. 296-305.

26. Leurs, M.T.W., et al., Integrale samenwerking gaat verder dan de samenwerking tussen professionals: introductie van het DISC-model Integrated collaboration continues beyond professional collaboration: introduction of the DISC-model. Tijdschrift voor Gezondheidswetenschappen Journal for Health Sciences, 2003. 81(6): p. 369-372.

27. Peters, L.W.H., et al., Ontwikkeling van de schoolSlag checklist voor kwaliteit van schoolgerichte interventies Development of the schoolBeat checklist for quality of schoolbased interventions. Tijdschrift voor Gezondheidswetenschappen, 2004. 82(1): p. 50-57.

28. Allensworth, D. and L. Kolbe, The comprehensive school health program: exploring an expanded concept. Journal of School Health, 1987. 57: p. 409-412.

29. St Leger, L.H., The opportunities and effectiveness of the health promoting primary school in improving child health - a review of claims and evidence. Health Education Research, 1999. 14(1): p. 51-69.

30. Judd, J., C. Frankish, and G. Moulton, Setting standards in the evaluation of community based health promotion programmes - a unifying approach. Health Promotion International, 2001. 16(4): p. 367-380.

31. Green, L.W. and F.M. Lewis, Measurement and evaluation in health education and health promotion. 1986, Palo Alto, CA: Mayfield.

32. Green, L.W. and M.W. Kreuter, Health Promotion and Planning: an educational and ecological approach. 1999, Mountain View: Mayfield Publishing Company.

33. St.Leger, L. and D. Nutbeam, Evidence of effective health promotion in schools, in The evidence of health promotion effectiveness. Shaping public health in a new Europe, D. Boddy, Editor. 1999, European Union: Brussels. p. 110-122.

34. Lister-Sharp, D., et al., Health promoting schools and health promotion in schools: two systematic reviews. Health Technology Assessment, 1999. 3: p. 22.

35. Rissel, C. and N. Bracht, Assessing Community Needs, Resources, and Readiness - Building on Strengths. Bracht, N (ed). Health promotion at the community level 2nd ed. Sage Publications, Inc.: Thousand Oaks, California, 1999.

36. Nutbeam, D., L. Aar, and J. Catford, Understanding childrens's health behaviour: the implications for health promotion for young people. Social Sciences and Medicine, 1989. 29(3): p. 317-325.

37. Leurs, M., Gezondheidsbevordering in het onderwijs: de schoolSlag-uitdaging Health promotion in education: the schoolBeat-challenge. Remediaal, 2003. 4(1): p. 3-8. 


\section{CHAPTER 5}

38. Boerma, L. and W. Hegger, Gezond op School - een methodiek om team en ouders samen te laten beslissen over prioriteiten op het gebied van gezondheid Health at School - a method for team and parents to prioritize regarding health. 2001, Nijmegen: ITS.

39. Marx, E. and S.F. Wooley, Health is Academic - A Guide to Coordinated School Health Programs. 1998, New York: Teachers College Press.

40. Vandenbroucke, S., F. Lenders, and J. Vinck, De kwaliteit van de planning van gezondheidspromotieprojecten in Vlaanderen The quality of the planning of health promotion projectects in Flanders/Belgium. Tijdschrift Gezondheidsbevordering, 1995. 16: p. 150162.

41. Ader, M., et al., Quality indicators for health promotion programmes. Health Promotion International, 2001. 16(2): p. 187-195.

42. Cameron, R., et al., Linking Science and Practice: Toward a System for Enabling Communities to Adopt Best Practices for Chronic Disease Prevention. Health Promotion Practice, 2001. 2(1): p. 35-42.

43. Molleman, G.R.M., et al., De Preffi 2.0: systematische ontwikkeling van een kwaliteitsinstrument voor gezondheidsbevordering The Preffi 2.0: systematic development of a quality instrument for health promotion. Tijdschrift voor Gezondheidswetenschappen Journal for Health Sciences, 2003. 81(5): p. 247-255.

44. Pratt, J., D. Pampling, and P. Gordon, Partnerships fit for pupose? 1998, King's Fund: London.

45. Walker, R., Collaboration and alliances: a review for VicHealth. Melbourne: VicHealth, 2000.

46. Mur-Veeman, I. and A. Van Raak, Interorganisational networks on the Dutch home health care market. International Journal of Health Planning and Policy, 1994. 9: p. 245-258.

47. Van Raak, A., et al., Integrated Care in Europe. Description and comparison of integrated care in six EU countries. 1st ed. 2003, Maarssen: Elsevier.

48. Ravensbergen, J., ZON, ZonMW en Effectieve Implementatie Zon, ZonMW and effective implementation, in In zicht - nieuwe wegen voor implementatie, J. Ravensbergen, et al., Editors. 2003, Koninklijke Van Gorkum: Assen.

49. Ruland, E., et al., Managing New Public Health: hoe zijn blijvende preventieve samenwerkingsverbanden te realiseren? Een agenda voor actie en onderzoek How can preventive collaborations be realised? An agenda for action and research. Tijdschrift voor Gezondheidswetenschappen, 2003. 81(1): p. 52-55.

50. Yin, R.K., Case study research. Design and Methods. 5th ed. 1994, Thousand Oaks: Sage Publications.

51. McLeod, J., The Partnership Analysis Tool: for partners in health promotion VicHealth, 2003.

52. Goodman, R.M., Principles and tools for evaluation community-based prevention and health promotion programs. Journal of Public Health Management and Practice, 1998. 4(2): p. 37-47. 


\section{CASE 5. OVERVIEW OF APPLIED PRACTICAL STRATEGIES}

\section{The administrative level}

In the year 2000 a study by the Southern Limburg GGD revealed high future prevalence estimates for mental health problems on the one hand, and inadequate regional mental health care supply in the near future on the other hand ${ }^{310}$. Based on this report, the managing director of the GGD, assuming the role of social entrepreneur, invited the heads of other agencies engaged in mental health care (Riagg Maastricht), social welfare (Stichting Trajekt), addiction care (Mondriaan Zorggroep), youth care (Bureau Jeugdzorg) and the local psychiatric hospital (Vijverdal) for a meeting. After two consultations, they agreed upon a general mission statement to collaborate in promoting public mental health, starting with young people. Participation by local authorities, in this case the alderman of Maastricht responsible for mental health care, was officially requested. However, since the alderman's department was at the time in the process of reviewing its policies on public mental health, the alderman postponed a decision, though indicating that she would like to be kept informed. The relevant civil servants were kept informed through personal contacts. A request to meet both the aldermen in charge of public health and education since youngsters of school age were the target population - remained unanswered and the issue did not reach the policy agenda during the preparation stage in 2001, despite lobbying. The municipal authorities of the Southern Limburg municipalities had agreed to act in concert as regards public health. As a result, the civil servants and aldermen of the neighbouring municipalities did not participate either. In 2004, a new alderman took office in Maastricht, who fully supported the Schoolbeat method, and from that moment on, the political agenda could be successfully influenced by the institutional managers [advocacy +- ], [agenda setting +- ]. Although a coalition between institutional managers was built, it did not have sufficient administrative power because the key players, i.e. the local authorities, were not represented, and the research domain was also absent, as the relevant university professor only participated at a later stage. The coalition was restricted to the participants' own niche and related to intentions only [coalition building -].

The intention was to set up a mental health programme in the school setting, combined with research to evaluate the evidence for its effectiveness. Since local grants were said not to be available, and all collaborating agencies were being confronted with budget cuts, it was agreed to submit a grant application to the Netherlands Organization for Health Research and Development (ZonMw, Healthy Living programme), under the condition that each agency was willing to critically reflect on and, if necessary, revise its health promotion activities, in order to increase the chances of success of the ZonMw application 


\section{CHAPTER 5}

[resource negotiations + ]. The ZonMw grant scheme conditions forced the institutional managers to submit their application before a given deadline, without having reached full agreement about its content, as the welfare agency still had some doubts and neither the Maastricht civil servants nor the alderman had reacted to the proposal before the deadline. When the grant for the Schoolbeat programme was finally awarded, the alderman of a neighbouring municipality was 'not amused', as she had not been informed. The crisis of confidence between this alderman and the GGD Board was slowly overcome by mediation and personal contacts between the managers and aldermen involved, and between the project leader of Schoolbeat and civil servants. Both during the preparation phase and the implementation phase, the institutional managers and the project leader invested heavily in lobbying activities. They initiated many consultations with policy makers, civil servants and members of political parties, even at the national level, to advocate the benefits of collaboration between policy, practice and research [sidestepping the formal system +].

\section{The institutional level}

Each of the institutional managers appointed a representative to participate in an intersectoral working group formed by partners from the practice domain. The research domain became involved at the request of the GGD. This working group wrote the grant application during an 8-month preparation period, meeting at least once a month and trying to find consensus. The problem diagnosis as regards collaboration between agencies from the practice domain and between the practice and research domains was part of the grant application, while the link with the policy domain was left aside, as this domain did not participate [diagnosis +-]. An action plan for the collaboration process was prepared, based on the assumption of joint contributions to school health improvement, again leaving the role of the policy domain undefined ${ }^{311}$ [activities and interventions +-$]$. The project leader identified the various stakeholders whose commitment was essential, and a strategy for gaining their support was implemented [commitment + ]. The ZonMw application was further worked out into an action plan, which clearly defined the management structure of the intersectoral collaboration, consisting of a steering committee, a project group, a national guidance committee and a research-practice advisory group. The latter was initiated to allow researchers and practitioners to inspire each other, and proved successful. This commitment strategy resulted in a positive attitude among the civil servants, inducing them to join the project group [intersectoral management structure +$]$. A communication plan was prepared, specifying a communication structure, time planning and the various activities, such as media attention, electronic newsletters and reports [communication structure + ]. The evaluation was assessed, in which the collaboration process was the object 
of study. The evaluation design was agreed upon by all parties involved [evaluation +]. Resources were made available in the form of staff provided by the research and practice partners, formalized in contracts quantifying the commitment in terms of content and/or full-time equivalents. The ZonMw grant was reserved for initial, innovative coordination beyond the collaborating partners. During the implementation phase, the project leader initiated negotiations to acquire additional resources, especially to allow better linkage between the research and practice domains and to allow the $\mathrm{PhD}$ thesis that was to be produced as part of the project to be completed within the required period [resources + ].

The mission statement was neither explicitly articulated nor formalized, but represented by the various communication media, in which the agreed goal and the concerted actions by schools, practitioners, researchers and policy makers were used to illustrate the benefits [mission statement +- ]. The communication strategy, including interviews with and activities by role models etc., evoked the impression of a learning collaborative partnership. Role models were recruited especially from the policy domain and the target population [interviews with role models + ]. The institutional management agreed upon internships and secondments, which resulted in a 'win-win' perception, as it led to insights into the other organizations and mutual service rendering. Interns from the Department of Health Promotion of the Maastricht University Faculty of Health Sciences contributed to knowledge development [internships +]. As recommended by the project leader, the institutional managers supported the development of a culture of collaboration by means of service rendering, access to each other's tools, and formal and informal consultation structures [rendering service, access to tools + ], [formal and informal consultations + ].

Regular assessments of the state of affairs with feed-back to the GGD management and the coalition partners were part of the coordinating tasks of the project leader [diagnosis and feedback + ]. Considerable investments were made to facilitate capacity building, as the mode of operation was completely different from what the collaborating partners had been used to so far. Performance indicators were defined for the function of programme advisors, with the aim of improving collaboration. A professional development policy was set up to overcome any lack of skills, which was accepted by the institutional managers. The policy domain, however, was not involved [performance indicators and professional development +-$]$. The ZonMw grant provided resources to appoint a project leader who was able to start the collaborative innovation process. Additional temporary staff was recruited during the initial phase [recruiting and retaining staff + ].

A kick-off conference with representatives of regional schools, practitioner services, policy makers and researchers was held ${ }^{312}$, followed by two other conferences, and a final conference in 2006 to present the handbook produced for 


\section{CHAPTER 5}

the project to local politicians [kick-off conference +]. Leadership qualities contributed to a working climate and culture that fitted in with the goals of the Schoolbeat programme, i.e., a collective approach involving the practice, research and policy domains and transcending the individual institutional goals. The leader frequently sidestepped the formal system to convince partner organizations of the benefits, to communicate the ongoing process and to solve any practical problems that occurred [sidestepping the formal system + ].

\section{The individual level}

Knowledge, attitude, perceived norms and outcome expectations were given much attention. The Schoolbeat method required an attitude and knowledge level that was rather different from what was generally accepted from these kinds of institutions. Individual professionals were asked to function as advisors in the programme. They were expected to advice schools about school health programmes available at the five collaborating partners and to promote positive attitudes by explaining the benefits of collaboration. Perceived social norms and outcome expectations had to change in favour of collaboration. The change process was facilitated by the formation of a working group, chaired by the project leader, which analyzed the starting position and gave regular feedback on the essential aspects [diagnosis and feedback + ]. This included sharing information on both content and process, receiving personal recognition, overcoming language barriers, increased networking, joint decision-making and consequently losing some institutional autonomy but getting other benefits in return [group discussion in multidisciplinary groups +]. Skills training sessions were organized to allow people to operate as advisors. A wide range of instruments was developed to support behavioural capabilities and self-efficacy in the daily work of the professionals, including quality assessment, priority workshops, school health profiles, and a 'Schoolbeat matrix' [learning by doing +], [detailed action plan, formats, materials + ]. The programme advisors formed a network to discuss barriers and facilitating factors related to their performance as advisors, indirectly enhancing their self-efficacy [feedback by peer networking + ]. Two professors of the local university were invited to hold lectures about school health promotion experiences abroad, encouraging the partners to further develop the Schoolbeat method [lectures and discussion + ]. The project team collaborated with NIGZ to offer master classes to disseminate the ideas underlying the programme and train professionals in the Schoolbeat method [master classes with skills training +]. The practitioners were coached to help them to write and submit articles. This led to a diversified publication strategy and conference contributions transcending the limits of their own niche, and about twenty-five articles were published in periodicals ranging from peer- 
reviewed scientific journals to professional bulletins [in-service coaching to publish beyond one's own niche + ].

\section{Comments}

The Schoolbeat programme was initiated by a group of institutional managing directors and was intended to be a long-term programme and multi-faceted in its focus on mental health. The lack of commitment from the policy domain at the start of the programme was disappointing, but after a new alderman took office at Maastricht in 2004, understanding between the practice and policy domains improved, and the provincial authorities also began to support the programme. The Schoolbeat case clearly shows the importance of linking individual, institutional and administrative changes that are necessary to achieve sustained collaboration. The Schoolbeat case study reveals that activities can still be provisionally carried out without support from the administrative authorities. However, public health practice needs to be embedded in policy to achieve effects in the long term. The policy work cycle was insufficiently involved during the innovation stage (2002-2006). Local policy makers have promised that, from 2006 onward, more direct links with local youth policy, educational policy, public health policy and public safety policy will be provided, to enhance the programme's effects on school health promotion. Recently, all partners have agreed on continuation of the process after 2006, though within the budgetary restrictions of the various collaborating partners. In view of the intended 10-year planning horizon there are another six years to go to make the Schoolbeat method an acceptable and routine method for school health promotion and to vigorously link the niche characteristics to achieve an evidence-based approach.

The Schoolbeat programme revealed the importance of support by a national research funding agency like ZonMw to invest in intermediate goals. The ZonMw application defined intermediate goals expressed in terms of organizational change and collaborative, intersectoral progress, rather than in terms of individual health outcomes, since health promotion activities have to run for many years before effects at individual level can be measured. The GGD, the National Institute of Health Promotion and Disease Prevention and the Department of Health Promotion of the Maastricht Faculty of Health Sciences jointly argued the need for accountability on intermediate goals. ZonMw's Healthy Living programme committee accepted the importance of intermediate process goals, which was rather revolutionary.

The Schoolbeat case also illustrates the struggle to combine practice with academic research. The programme was always intended to result in a $\mathrm{PhD}$ dissertation linking practice to research. Research issues were introduced, resulting in complex interconnections between work cycles, in which various 


\section{CHAPTER 5}

research issues entered the practice cycle and vice versa. For instance, research into quality assessment of school interventions was carried out according to the research cycle, after which the results were fed into the practice cycle. Research on the collaborative working process included action research, which paralleled the practice work cycle. Combining the hectic practical work situation with the project leadership and in-depth academic research proved to be too laborious a task for one person. In future projects, conditions will have to be created that would make such an effort feasible.

So far, the intention to achieve evidence-based quality in school health promotion through collaboration has appeared feasible. First, the quality checklist which was developed for school health promotion programmes is serving the programme advisors in their task to help schools prioritize their plans. Based on this checklist, priority can be given to evidence-based programmes. The development of this quality checklist would not have been possible without the concerted efforts of the research and practice domains. Secondly, 'priority workshops' have been developed through collaboration between the Schoolbeat partners and the schools. This instrument allows schools to prioritize issues for health programmes, in close cooperation with parents, teachers and students. After it has shown its feasibility in practice, this instrument will need further research to prove its effectiveness in term of school empowerment, participation level and effect parameters. This research will be undertaken by the Academic Collaborative Centre for Public Health. Thirdly, the Schoolbeat collaboration may have contributed to the formation of links between education policy and public health policy, although it is impossible to indicate what specific practical strategies have contributed to such links. As policy domains normally work isolated from each other, the rapprochement between the departments of education and public health can be considered as rather revolutionary. These 'revolutionary' steps need further development, in combination with research to measure their effects on school health promotion. Finally, the evaluation of the collaboration process, i.e., the DISC model, has been developed through joint actions by the research and practice domains. It may serve as an eclectic tool to monitor the collaboration between policy, practice and research, in order to assess the progress in school health promotion.

The project's success in linking the niches has amongst others been due to passionate leadership, which allowed many different practical strategies to be simultaneously applied at the various system levels. The Schoolbeat method evolved as a national model, being disseminated by the Healthy School Centre of the National Institute of Health Promotion and Disease Prevention ${ }^{313}$. The above case study allows us to conclude that links between the system levels are a prerequisite to initiating and sustaining collaboration. We also found that the application of many different practical strategies supported the collaboration 
process. The investment in intermediate goals like collaboration process indicators, instead of ultimate health outcomes, needs to be considered at the national level, as it may induce collaboration between specific partners, not just for the sake of collaboration, but to address certain public health problems. 


\section{CHAPTER 5}

\subsection{CASE 6. INTRODUCTION}

Continuation processes for a successful preventive intervention: Heartbeat 2. The concept of sustainability and the use of outcome indicators

It was the ambition of this collaborative project to develop a comprehensive prevention programme on cardiovascular diseases, to investigate its effects, and finally to achieve sustainable implementation by intersectoral collaboration in order to strengthen public health policy for this topic. The programme, called Heartbeat Limburg (Hartslag Limburg in Dutch), was launched in 1998. The intersectoral collaboration was achieved through a close-knit network of all participating institutions, ranging from local authorities, sports associations, welfare agencies, schools and home care agencies to volunteers and community centres.

Heartbeat combined a population-based strategy, intended especially for low socio-economic status groups (the community approach), and a high-risk strategy for patients diagnosed with cardiovascular disease or having multiple risk factors ${ }^{314}$. Between 1998 and 2003, a total of 852 activities were organized, ranging from healthy cooking, walking, line dancing, aerobics, yoga and smoking cessation projects to guided tours in supermarkets, and cheap and healthy food for people with severe debts. High-risk patients were recruited at the Maastricht University Hospital and in general practice. Patients participated in an individual lifestyle counselling programme to motivate them to manage a healthy lifestyle themselves, tailored to their own preferences and demands.

A thorough research plan accompanied the implementation, to study the programme's effectiveness, efficacy, cost-benefit ratio and ethical consequences. The programme proved to be effective at the population level ${ }^{315-317}$. More detailed results have been presented in various relevant publications ${ }^{318-333}$. The Heartbeat programme involved a number of phases, viz. the preparation phase (1994-1998), the implementation phase (1998-2002), the continuation phase (2002-2006) and finally, from 2006 onwards, the institutionalization phase.

After the pilot in 2002, various administrative and institutional constraints meant that only the high-risk strategy for hospital patients could be continued, supported by a grant from the Dutch Public Health Care Fund (Fonds OGZ). The lifestyle programme continued under the name of Heartbeat 2, to express support for the two-pronged-strategy approach (i.e. community and high-risk). The article included below describes the barriers and facilitators of the Heartbeat 2 lifestyle programme during its transition from the implementation to the institutionalization phase. The assessment of the application of practical strategies to promote collaboration is restricted to the 1994-2002 period, i.e., the Heartbeat 1 programme. 
Submitted to: Health Promotion International; date of submission: 14 January 2007

Continuation processes for a successful preventive intervention: Heartbeat 2 . The concept of sustainability and the use of outcome indicators.

Maria Jansen, MSc, programme manager of the Academic Collaborative Centre for Public Health Limburg, PHS South Limburg, the Netherlands

Janneke Harting, Assistant professor at the Department of Health Education and Promotion, Maastricht University, Maastricht, the Netherlands

Nicole Ebben, MPH, research assistant, PHS South Limburg, the Netherlands

Bram Kroon, MD, assistant professor at the Cardiovascular Centre, Maastricht University Hospital, Maastricht, the Netherlands

Jan Stappers, MD at the Cardiovascular Centre, Maastricht University Hospital, Maastricht, the Netherlands

Esther van Engelshoven, MSc, vice-director of the Cardiovascular Centre, Maastricht University Hospital, Maastricht, the Netherlands

Nanne de Vries, Professor at the Department of Health Education and Promotion, Maastricht University, Maastricht, the Netherlands 


\title{
CHAPTER 5
}

\begin{abstract}
In order to ensure the continuation of a successful pilot programme, routinisation of working methods in daily practice must take place. This article describes the change process of Heartbeat 2, a health counselling programme for high risk cardiovascular patients. In particular, this article focuses specifically on matters relating to the continuation and sustainability of the programme. In order to study the extent to which the programme is sustainable, the change agent used outcome indicators based on the following questions: Why should health counselling be sustained? How should this be done and by whom? How much needs to occur and by when? The results indicate a need for a linkage system in the final stages of change so that the programme is maintained. Limitations of the external change agent are described. The outcome indicators appeared to be an adequate operationalization to monitor sustainability. The change process leading up to sustainability appeared to be highly complex and was complicated even further by unpredictable and unforeseen external factors. Contrary to our expectations, the continuation of Heartbeat 2 did not provide sufficient evidence that sustainability had occurred. We conclude that sustainability is an ongoing, cyclic evolvement that occurs through a social process of human interactions.
\end{abstract}

Keywords: health counseling, sustainability, change agent, programme incorporation, capacity planning

\section{INTRODUCTION}

Empirical knowledge about the sustainability of health behaviour change programmes in health care settings is limited ${ }^{1-6}$. Sustainability is a complex process $^{7-13}$ that should be developed and assessed over a period of 10 to 15 years, involving a range of short medium and long term programme outcomes ${ }^{14-16}$. Sustainability has been described as the final stage of programme use in which the programme is incorporated into organisational routines so that it will be maintained once the original programme funding, adopters, or programme champion are no longer present $t^{17}$. So far, most theories emphasize the process of adoption and pilot implementation rather than explaining how complex organisations solve problems related to the integration of innovations into normal functioning. However, some attempts at modelling effective sustainability have been made. First and foremost, Shediac-Rizkallah and Bone ${ }^{18}$ describe three determinants of sustainability. The first is health benefits, which are at the heart of the process since interventions that yield no health benefits are not worth sustaining. The second is institutionalization factors as these are necessary to perpetuate the programme itself. The third determinant concerns com- 
munity capacity attributes as these attributes can influence sustainability processes at the societal level. Pluye ${ }^{19}$ claims that, at the organisational level, routines and standards (as defined in terms of memory, adaptation, values and rules) are also conducive to normal functioning in the organisation.

According to diffusion of innovations theory (see box 1), the change process is characterised by a continuous shift that starts with the initial adoption of an idea and then moves on to the implementation of the change, the maintenance of that change and, lastly, the institutionalization of the change. This process can be facilitated by a change agent, who functions as a link between the resource system and the user system ${ }^{20-27}$. The resource system is defined as the organisation that develops the innovation and the user system is the group of individuals that makes use of the innovation. Change agents may come from either of the two systems. Since the main role of the change agent is to create a dialogue, which requires impartiality, the background should not affect the change agent's role ${ }^{24}$. Research on the effectiveness of both the change agent and the linkage function during the sustainability stage has not yet been reported. Therefore, this article endeavours to provide some insight into whether, and how, a linkage system and a change agent can promote sustainability. Additionally, the practical value of monitoring the degree of sustainability by means of the three indicators mentioned above (individual health benefits, the institutionalization process and the available capacity) is also discussed.

In this study, the Heartbeat 2 (in Dutch: Hartslag 2; 2003-2006) ${ }^{28}$ project is used as a case study. Heartbeat 2 evolved out of an earlier project entitled Heartbeat Limburg (in Dutch: Hartslag Limburg, see box 2) ${ }^{29}$. The core component of Heartbeat 2 is the integration of health counselling ${ }^{30}$ into the regular medical treatment for cardiovascular patients at Maastricht University Hospital's Cardiovascular Centre (HartVaatCentrum azM, hereafter referred to as CVC $)^{31}$. Based on the cardiovascular risk profile special trained health advisors discussed the patient's risk behaviours trying to motivate the patient to change lifestyle. Given that most health care systems emphasize the treatment of disease rather than the promotion of health ${ }^{32-34}$, many barriers exist with respect to the routinisation of preventive health counselling in hospitals. This is the case even when a relatively successful pilot phase has been completed (see box 2).

Since the first Heartbeat Project generated promising results ${ }^{35-37}$, the board at the CVC decided to continue to provide the health counselling service. However, because other studies have generated mixed results $38-55$, long term follow-up was needed to convince all staff involved. Additionally, the reinvention of health counselling was seen as a means to create clear task assignments for cardiovascular nurses and health advisors with regard to health counselling. Integration or specialization of the two jobs would fit well with the concept of task substitution, in which tasks of specialists are transferred to cardiovascular 


\section{CHAPTER 5}

nurses. The CVC thus considered an investigation of the effects of this reinvented health counselling necessary.

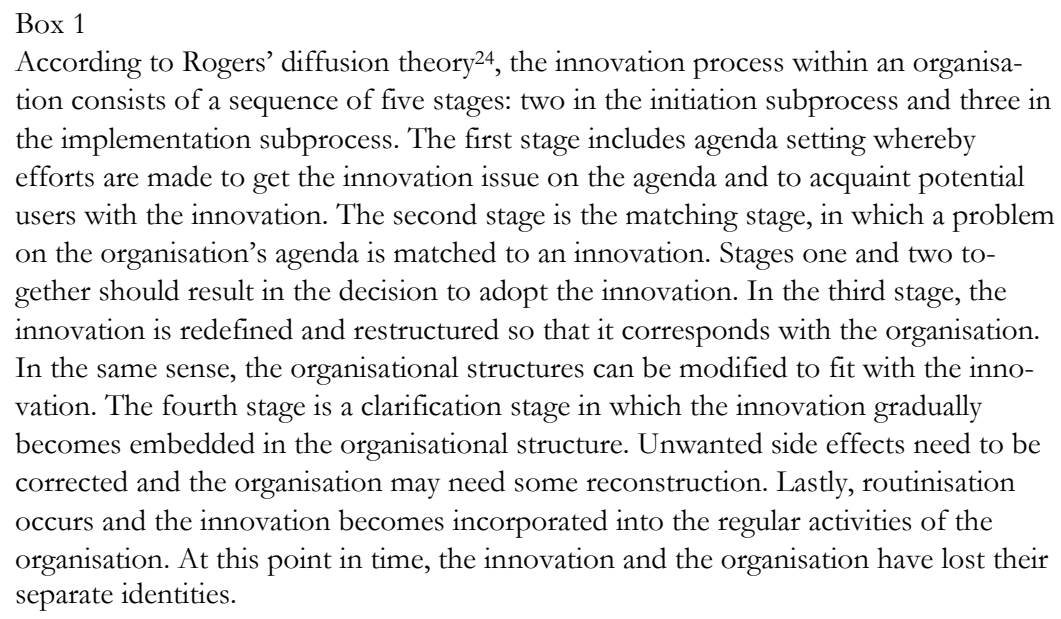

\section{IMPLEMENTATION: STRATEGY AND INDICATORS}

In order to support the process of creating sustainability a linkage system between the CVC (the user system) and the PHS (the resource system) was built ${ }^{17,21,22,24}$. The project leader of the PHS was appointed as an external change agent. The change agent built the linkage system by organising a steering committee. The steering committee was chaired by the vice-director of the $\mathrm{CVC}$ and further consisted of a cardiologist (CVC), an internist (CVC), three health advisors (CVC), a research assistant (PHS), and the project leader/change agent (PHS).

The change agent was primarily concerned with determining the main outcome indicators for success, by providing answers to the following questions: 1) Why should the health counselling programme be sustained?; 2) How can this be done?; 3) By whom should this be done?; 4) How much has to be done?; and 5) By when should sustainability be achieved? ${ }^{28,56}$. The first question served as a prerequisite for the latter questions. It was necessary to determine if health counselling was indeed effective in real-life practice. This was done by examining whether new clients did experience health benefits. The second and third questions were necessary in order to determine how health counselling could best be routinised in disease management programmes. The final two questions had to be answered to determine the level of capacity needed in order to actually realise the routinisation. By answering these questions, outcome indicators 
could be determined. These outcome indicators were then used as: a) the change agent's objectives; and b) the actual assessment of the programme's sustainability.

Box 2

Heartbeat 2 evolved from the Heartbeat Limburg project (in Dutch Hartslag Limburg, 19982003 ${ }^{29}$. Heartbeat Limburg was a regional project that endeavoured to prevent cardiovascular disease (CVD) in the southern part of the Dutch province of Limburg.

Heartbeat Limburg consisted of a community and a high-risk approach, whereas Heartbeat 2 only continued the high-risk approach in the CVC of the university hospital. The high risk approach focused on individuals who were at elevated risk for developing CVD. These high-risk individuals underwent a cardiovascular health check by their cardiovascular specialist. Based on the patient's risk profile, health counselling was offered whereby cardiovascular risk behaviours could be discussed and possible lifestyle changes could be recommended. The new function of health advisor was created to implement health counselling.

Between 1998 and 2001, a randomized controlled trial was conducted. The study revealed the existence of short term (after 4 months) programme effects on fat consumption, smoking, physical activity and the intention to adopt a healthier lifestyle. After 18 months, no effects, other than an increased intention to reduce fat intake, were found ${ }^{46}$.

Furthermore, the findings of the trial showed that the content of the health counselling did not match the needs of $12 \%$ of the patients ${ }^{35,37}$. Especially for those recently discharged from the hospital, stress, worry, anxiety, and uncertainty about medication were much more important topics than (other) lifestyle issues. The innovation was thus 'reinvented' between 2001 and 2003 so that counselling would also cover the issues relating to aftercare for patients discharged from hospital and topics relating to uncertainties and anxiety in patients' home situation.

A post-trial implementation study was conducted from 2001 until 2004. The study indicated that the adoption of the innovation at the cardiology outpatient clinic had been successful ${ }^{36}$.

From 1998 to 2003, a full-time equivalent (fte) health advisor was appointed to carry out health counselling. High-risk patients were invited by the cardiology outpatient clinic to participate. Since the start of the Heartbeat 2 programme in 2003 , a 1.7 position was made available and patients were invited by the CVC to participate. The CVC is a collaboration between the departments of cardiology, vascular neurology, vascular medicine, vascular surgery, and cardiothorax surgery. For each department, an integrated disease management programme will be realized.

The change agent attempted to answer the first question (Why?) by implementing a surveillance system that could continuously measure the health benefits experienced by new clients. The initial plan was to administer Risk Consult ${ }^{\circledR}$, a computerized cardiovascular risk profile programme, at the CVC outpatient clinic. Unfortunately, hospital-wide budget-cuts resulted in the delayed implementation of the system. Patient records, including a short questionnaire that had been administered by the health advisors, were thus used for the time period prior to the implementation of Risk Consult ${ }^{\circledR}$. The change agent also made arrangements to have two interns from the Faculty of Health Sciences at 


\section{CHAPTER 5}

University Maastricht test the inter-rater reliability of the administrators of the questionnaire, which proved sufficient. The change agent also held a series of in-depth interviews with patients to determine patient preferences for maintaining lifestyle adjustments (i.e., physical activity) in their daily routine.

In order to answer the second and third questions (How? By whom?), the change agent initiated discussions about the reinvention of health counselling as part of the new disease management programmes. The change agent determined that a referral and follow-up procedure in the disease management programmes with clear performance standards for the procedures was needed.

In an attempt to answer the final two questions (How much? By when?), the change agent created an inventory of the current total capacity of cardiovascular nurses, health advisors and patient consultations within the CVC. Given that health counselling was to be implemented in five disease management programmes, the change agent determined that more capacity was required within existing budgets. A task division was created between health advisors and cardiovascular nurses that was compatible with the concept of task substitution, that was matched to the number of patients, and that could result in a sustained health counselling programme. Between 2003 and 2006, the available capacity increased from 1.0 to 1.7 full time equivalents, enabling the counselling of 500 patients per year (approximately $10 \%$ of the eligible cardiovascular patient population)

In summary, the strategies applied by the change agent included: linking the resource and user system through the steering committee (see table 1, strategy 1$)$, measuring the health benefits experienced by new patients ( $2 a, 2 b)$, discussing the possibilities of making health counselling a routine part of the disease management programmes ( $3 \mathrm{a}, 3 \mathrm{~b})$, compiling an inventory of capacity requirements (4a, 4b, 4c), and communicating to attain CVC-staff commitment for an ongoing health counselling programme within the disease management programmes beyond 2006 (5a, 5b, 5c).

To study the effectiveness of change agent's strategies and the linkage system to promote sustainability, we measured the outcome indicators of the why, how, by whom, how much and by when questions.

\section{METHODS: DATA COLLECTION}

Data were derived from documents, minutes, progress reports, interns' reports, health advisors' patient records, CVC Board statements and focus group interviews. The quantified characteristics with regard to the linkage system include: number of meetings, rate of participation, topics discussed, bilateral consultations initiated by the change agent, training and communication expressions. The degree to which the change agent contributed to an increased commitment 
to health counselling was assessed using focus groups and by referring to the CVC Board's final decisions. In 2006, five focus group interviews of approximately one hour were conducted in order to list members' experiences with the adoption, implementation and maintenance phases. The focus group interviews were conducted with a total of $23 \mathrm{CVC}$ managers, specialists and nurses. Each group consisted of five to eight CVC staff members that were randomly selected from the total CVC team $(n=151)$. Every group was led by the same moderator, namely the PHS research assistant, who made use of a predetermined format ${ }^{57}$. All interviews were tape-recorded and transcribed in the code system of QRS NVivo.

Table 1 Number of strategies applied by change agent

\begin{tabular}{|c|c|c|c|c|}
\hline Applied strategies & 2004 & 2005 & June 2006 & Total \\
\hline 1a. formal meetings steering committee & 8 & 8 & 5 & 21 \\
\hline $\begin{array}{l}\text { 2a. discussions about surveillance with } \\
\text { Risk Consult }{ }^{\circledR}\end{array}$ & 7 & 8 & 4 & 19 \\
\hline $\begin{array}{l}\text { 2b. discussions about monitoring long } \\
\text { term effects on lifestyle }\end{array}$ & 3 & 2 & 2 & 7 \\
\hline $\begin{array}{l}\text { 3a. discussions about where to incorpo- } \\
\text { rate health counselling in the disease } \\
\text { management programmes }\end{array}$ & 4 & 7 & 4 & 15 \\
\hline $\begin{array}{l}\text { 3b. discussions about how to improve } \\
\text { patient referral }\end{array}$ & 2 & 2 & 1 & 5 \\
\hline $\begin{array}{l}\text { 4a. discussions about capacity and task } \\
\text { division }\end{array}$ & 1 & 3 & 2 & 6 \\
\hline $\begin{array}{l}\text { 4b. discussions about sustaining health } \\
\text { counselling as part of the disease } \\
\text { management programmes }\end{array}$ & 1 & 5 & 3 & 9 \\
\hline $\begin{array}{l}\text { 4c. discussions about the number of } \\
\text { counselled patients ( }>500 \text { a year) }\end{array}$ & 3 & 3 & 4 & 10 \\
\hline $\begin{array}{l}\text { 5a. communication activities to attain } \\
\text { commitment from a broad audience }\end{array}$ & 10 & 10 & 3 & 23 \\
\hline 5b. bilateral meetings & 12 & 6 & 4 & 22 \\
\hline $\begin{array}{l}\text { 5c. discussions about resources to main- } \\
\text { tain Heartbeat }\end{array}$ & 13 & 6 & 8 & 27 \\
\hline
\end{tabular}




\section{CHAPTER 5}

\section{RESULTS}

\section{Linkage system}

The steering committee met 21 times, six to eight times per year with a mean participation of 5.4 out of the 6 members. The atmosphere was positive. Partners were willing to discuss potential methods to reduce barriers.

In addition to the steering committee meetings, the change agent initiated 22 bilateral consultations about related topics. A total of nine columns (rolemodel articles) were published in the hospital magazine. Six national conference contributions as well as seven local presentations, of which three were addressed to the board of the CVC, were made. These contributions were made in order to link internal and external hospital staff to propagate preventive health counselling in hospitals.

\section{Sustainability: health benefits}

The pre-test and two post-test comparisons indicated a short term decrease in fat consumption (2003: $\mathrm{p}<.001 ; 2004: \mathrm{p}<.001)$ which did not sustain at the long-term. However, both a short term and a long term increase in physical activity was noted (2003 and 2004: $\mathrm{p}<.001)$. Maintenance of sufficient physical activity could be further supported by making physical activity fun, by supplying tailored services, by making self-management instruments accessible, and by helping patients overcome lapses ${ }^{58}$. The results also indicated that, in 2004, patients experienced both a short term $(\mathrm{p}<.001)$ and a long term $(\mathrm{p}<.05)$ reduction in stress. However, this was not the case in 2003. Smoking cessation did not sustain ${ }^{59,60}$. Two thirds of the patients $(69 \%)$ reported benefits from health counselling, while $22 \%$ were neutral and $9 \%$ reported no benefits. Health counselling topics that were perceived as either important or very important were, in successive order: aftercare and adherence to medication (73\%), physical activity $(65 \%)$, food and fat consumption (62\%), stress (57\%), and smoking $(52 \%)$. Approximately half of the patients reported many difficulties with respect to initiating and maintaining lifestyle changes. Health counselling service was rated at 7.5 on a ten point scale ${ }^{60}$. The results on smoking (negative), fat consumption (small) and physical activity (positive) were mostly consistent with the initial trial. However, the positive effects of counselling on physical activity were larger than expected ${ }^{36}$.

These results were received differently by the various CVC staff members. Approximately half of the staff members evaluated the results as making a very important additional contribution to patient treatment while others were not convinced that health counselling offers a surplus value to medical treatment programmes. The diversity of opinions could not be overcome. 


\section{Sustainability of programme activities: how and by whom}

Budget cuts had a considerable impact on the development of the disease management programmes. Instead of the intended 13 programmes, only 5 were realized, and only four of the six cardiovascular nurses were actually appointed. The cardiovascular nurses that carried out the diagnostic screening were supposed to refer patients to the health advisor ${ }^{61,62}$. However, because of delays in the implementation of the disease management programmes, many referrals were made by specialists, who had high rates of non-compliance. Since a referral and follow-up procedure with clear performance standards can help to assure the routinisation of health counselling in the disease management programmes, the change agent actively endeavoured to have this implemented. However, this aspect was not formally assessed by the management. Furthermore, neither cardiovascular nurses nor specialists systematically referred faithfully and systematically.

For a special group of patients (stable patients with coronary disease), a proposal was made to have the referral procedure connected to the concept of task substitution. The proposal was an effort to extend the health advisor's task to cover cardiovascular nursing duties ${ }^{63,64}$. Both cardiovascular nurses and health advisors were expected to record changes in biomedical risk factors, to inform the cardiologist about these risk factors, to make inventories of lifestyle risk factors, and to offer health counselling to patients. The first two tasks were added to the original health counselling programme and the reinvented health counselling was then embedded in the disease management programmes for stable coronary disease patients.

\section{Sustainability of capacity: how much and by when}

We planned to offer 1250 patients health counselling between 2003 and 2006. Finally, health counselling with $923(74 \%)$ patients was realized. Our intention is to increase this number in the coming years. In order to do this, it was necessary to obtain insight into referral and counselling procedures with clear performance standards for each professional in the chain of care, an estimation of the time needed to carry out the tasks, and a calculation of total capacity based on number of patients. This took a significant amount of time. Substitution of tasks required convergence between the function of cardiovascular nurse and health advisor. This convergence of functions was seen as a reason to terminate the employment of temporary part time (0.7) staff once external funding stopped. The steering committee decided to initiate a training programme on health counselling in order to increase total capacity. The change agent provided a budget for the years beyond 2006 whereby cardiovascular nurses could be trained in health counselling techniques. The health advisor function was thus integrated into the function of cardiovascular nurse. 


\section{CHAPTER 5}

The Board further determined that highly complex patients should be offered health counselling in the CVC-university hospital, moderately complex patients should be offered counselling in the CVC-medical centre where stable patients are monitored, and patients with low complexity should be offered counselling by nurses in general practice. The Board further contended that the investments should be divided according to this differentiation of patient categories.

\section{Commitment}

The results of the focus group interviews indicated that $39 \%$ of the CVC members were acquainted with Heartbeat, 39\% had heard about Heartbeat but knew very little about it, and $22 \%$ had not heard of Heartbeat at all. CVC members unanimously agreed that health counselling and lifestyle recommendations should be provided to cardiovascular patients. However, there were mixed opinions about who should provide this kind of counselling. Opinions on whether the CVC, the PHS or another organisation should provide the health counselling service were dependent on one's ideas and vision with respect to the core services and financial responsibilities of the above mentioned organisations. Despite divergent ideas on responsibilities, CVC members did agree that health counselling could be implemented by using the concept of task substitution. As a general rule, the cardiovascular specialist is the person who holds the primary responsibility for informing the patient about the disease and advising the patient to make lifestyle changes. CVC members all agreed that this task could be transferred to nurses. Either the health advisor's task could be extended to that of the cardiovascular nurse, or vice versa.

Practical strategies initiated by the change agent, such as role-model stories and conference contributions, had only a modest impact on commitment given that publications and conference contributions tend to reach groups others than the target population within the CVC.

CVC members criticized the fact that no supervisor was formally assigned responsibility for health counselling activities. Installing a supervisor somewhere between the health advisors and the vice-director could have strengthened managerial and administrative support. The members indicated that this kind of support is necessary for the effective assessment of performance standards, for task division, and for the adaptation of health counselling in such a way that it matches CVC disease management developments. In essence, CVC members saw the Heartbeat project as mainly supported by an external change agent with expert and informational power but without legitimate power related to the CVC.

Financial constraints were perceived as unchangeable. The CVC members believed that financial constraints force the $\mathrm{CVC}$ to focus on its core business, namely treating diseases. They further claimed that the continuation of health 
counselling can only be fully assured if health insurance companies take responsibility for the provision of preventive services in the health care settings.

The research period in this study coincided with Dutch health care reforms that aimed to introduce market principles into the health care system. Negotiations with health care providers by health insurance companies were required to ensure the delivering of high quality services at a low price. The system by which price is calculated had to be converted to Diagnosis Treatment Combinations (DBCs in Dutch). These developments led to a high level of uncertainty about the financial future of health care. This, in turn, made it rather inconvenient for CVC staff to decide on whether health counselling should be incorporated in the new disease management programmes, especially since the provision of health counselling could make the DBC for cardiovascular treatment more expensive than elsewhere.

\section{DISCUSSION}

The linkage system was indispensable for agenda setting reasons. It not only helped to maintain on ongoing discussion, it also helped to develop a shared vision and to create dialogue between the change agent and various levels of organisation in the hospital. It is likely that the ongoing discussions contributed to both a broader acceptance of the need for health counselling and attempts to combine the tasks of health advisors with the tasks of cardiovascular nurses. We thus conclude that our findings confirm the importance of a linkage system during the stage of attaining sustainability as emphasized by theory.

The impact of the external change agent shows mixed results. Roger's theory ${ }^{24}$ emphasizes that the position of the change agent should be in both the resource and the user system. It appears that, in our study, attaining this double position was very time consuming and whether we were successful in doing this remains unclear. A change agent from the resource system had been operating since 1998 and had been successful in realizing adoption, pilot implementation, and reinvention. The external change agent that functioned between 2003 and 2006 had been partly successful. Commitment increased but financial constraints were counterproductive. The change agent devoted a significant amount of time to the diagnosis of problems and the development of practical strategies for the reduction of perceived barriers, within the legitimate competences. In our opinion, the absence of an internal supervisor combined with the external change agent's lack of legitimate power hampered the formalisation of procedures that were essential to embed health counselling into the routines of disease management programmes. This finding suggests that an external change agent may need a counterpart from the user system during the sustainability phase, especially when it comes to influencing the management's decision- 


\section{CHAPTER 5}

making processes. The CVC has recently recognized this need for a supervisor and thus appointed one in August 2006. Additionally, we contend that the preference and effectiveness of an internal or external change agent during the sustainability phase needs to be investigated further.

Our case study reveals that the method used to monitor levels of sustainability, namely the use of medium and long term outcomes based on why, how, by whom, how much and by when questions, appears to be an adequate tool for unravelling the complexity of a change process. The answers to these questions provided sufficient information on the level of sustainability. In terms of Pluye's ${ }^{19}$ four degrees of sustainability, namely absent, precarious, weak, and routinised, Heartbeat can be characterized as a weakly sustained innovation. This means that, although the programme is officially sustained, the activities were not routinised and may thus be subject to radical changes in the short term.

Decisions on the definitive continuation or institutionalisation of a programme will be influenced by arguments other than those for temporarily pilot implementation. Roger's theory predicts that innovations that cause more 'irreversible changes' are less likely to be adopted. The adoption of a continuous programme could have been perceived as an irreversible change. Whether this kind of change is interpreted as a threat or an opportunity obviously impacts the decision to adopt. Because the disease management programmes in this study still had to be developed and a clear vision on most of these programmes was lacking, the CVC was unwilling to make definitive choices with respect to the health counselling service. In contrast, the disease management programmes for stable coronary patients had a clear vision on task substitution. The reinvented health counselling was seen as an opportunity to do this and thus generated a dynamic decision to incorporate health counselling.

Our findings also reveal the influence of unpredictable and unforeseen external factors, such as budgetary cuts and an increase in attention to task substitution. This finding corresponds with the organisational theory of contingency ${ }^{27}$, which postulates that organisational design reflects the degree of complexity of the environment in which an organisation operates. The research period coincided with Dutch health care reforms which resulted in a high degree of financial uncertainty. As a result, health insurance companies were not yet prepared to finance health promotion activities within curative health care settings. Nonetheless, despite the widespread dissemination of guidelines on cardiovascular risk management and self-management of chronic illness ${ }^{65-67}$, the CVC has yet to fully integrate these guidelines into practice. We found, in contrast to Pluye's theory ${ }^{11,19}$, that the availability of an institutional standard did not sufficiently support or convince the CVC to sustain health counselling. Compliance with guidelines or standards is thus unsatisfactory ${ }^{68,69}$. 
We initially considered sustainability to be the final phase of the innovation process. However, in accordance with the Scheirer ${ }^{56}$ findings, our case reveals that parts of the original health counselling were sustained while other parts were reinvented. The social environment in hospitals is constantly changing. Health policy and the structure of the hospital as an organisation are thus constantly in reform. We may thus conclude that sustainability is not simply the last phase of a change process but rather a much more ongoing, cyclic evolvement that functions through a social process of human interactions $4,24,70-72$. Much work has to be done over the course of the coming years before health counselling is a routine in hospitals.

The study covers only one descriptive case. Consequently, the extent to which the results can be generalised is limited. In this case study, questions of 'how' and 'why' were posed, the investigator had little control over events, and the focus was placed on a contemporary phenomenon in a real-life context ${ }^{73}$. As a result, the findings of our study may only allow for tentative conclusions. We conclude that the contribution of the linkage system and the change agent may be essential but unfortunately lacked a counterpart from the user side. Furthermore, the monitoring of medium and long term outcomes based on the why, how, by whom, how much and by when questions appeared to be an effective instrument for determining the extent and complexity of sustainability. Evidently, our study has shown that sustainability is not easily obtained. The meaning of the innovation is constructed over time to better correspond with the changing context. It may be a comforting thought that continuous developments of preventive health care innovations reflect continuous attempts to improve patient care.

\section{References}

1. Botvin GJ. Advancing prevention science and practice: challenges, critical issues, and future directions. Prevention Science 2004;5(1):69-72.

2. Glasgow RE, Lichtenstein E, Marcus AC. Why don't we see more translation of health promotion research to practice? Rethinking the efficacy-to-effectiveness transition. American Journal of Public Health 2003;93:1261-7.

3. Glasgow RE, Vogt TM, Boles SM. Evaluating the public health impact of health promotion interventions. The RE-AIM framework. American Journal of Public Health 1999;89:1322-7.

4. Johnson K, Hays C, Center H, Daley C. Building capacity and sustainable prevention innovations: a sustainability planning model. Evaluation and Program Planning 2004;27:135-49.

5. Oldenburg BF, Sallis JF, French ML, Owen N. Health promotion reserach and the diffusion and institutionalization of interventions. Health Education Research 1999;14:121-30.

6. Pentz MA. Form follows function. Designs for prevention effectiveness and diffusion research. Preventive Science 2004;5:23-9.

7. Greenberg MR. The diffusion of public health innovations. American Journal of Public Health 2006;96:209-10. 


\section{CHAPTER 5}

8. Health Council of the Netherlands. From implementation to learning: the importance of a two-way dialogue between practice and science in health care. Publication no. 2000/18. The Hague: Health Council of the Netherlands, 2000.

9. Plsek PE, Greenhalgh T. The challenge of complexity in health care. British Medical Journal 2001;323:625-8.

10. Plsek PE, Wilson T. Complexity, leadership, and management in health care organisations. British Medical Journal 2001;323:746-9.

11. Pluye P, Potvin L, Denis JL, Pelletier J. Program sustainability: focus on organizational routines. Health Promotion International 2004;19:489-500.

12. St Leger L. Questioning sustainability in health promotion projects and programs. Health Promotion International 2005;20:317-9.

13. Swerissen H, Crisp BR. The sustainability of health promotion interventions for different levels of social organization. Health Promotion International 2004;19(1):123-30.

14. Canadian Health Service Research Foundation. Weighing up the evidence. Making evidence-informed guidance accurate, achievable, and acceptable. Ottawa: CHSRF, 2006.

15. Lomas J, Culyer T, McCutcheon C, McAuley L, Law S. Conceptualizing and combining evidence for health system guidance. Ottawa: Canadian Health Service Research Foundation, 2005.

16. Whitelaw S, Baxendale A, Bryce C, Marchardy L, Young I, Witney E. 'Settings' based health promotion: a review. Health Promotion International 2001;16(4):339-52.

17. Bartholomew LK, Parcel GS, Kok G, Gottlieb NH. Planning health promotion programs. An intervention mapping approach. 2nd ed. San Francisco: Jossey-Bass, 2006.

18. Shediac-Rizkallah MC, Bone LR. Planning for the sustainability of community-based health programs. Conceptual frameworks and future directions for research, practice and policy. Health Education Research 1998;13:87-108.

19. Pluye P, Potvin L, Denis JL. Making public health programmes last: conceptualizing sustainability. Evaluation and Program Planning 2004;27:121-133.

20. Fleuren MAH, Wiefferink CH, Paulussen TGW. Determinants of innovations within health care organizations. Literature review and Delphi-study. International Journal for Quality in Health Care 2004;16:107-23.

21. Lomas J. Using 'Linkage and Exchange' to move research into policy at a Canadian Foundation. Health Affairs 2000;19:236-240.

22. Orlandi MA. Clinical perspectives. Promoting health and preventing disease in health care settings: an analysis of barriers. Preventive Medicine 1987;16:119-30.

23. Raad voor de Volksgezondheid en Zorg. Van weten naar doen. Zoetermeer: Raad voor de Volksgezondheid en Zorg, 2005.

24. Rogers EM. Diffusion of innovations. 5th ed. New York: Free Press, 2003.

25. Schrijvers AJP. Tien theorieën om de snelheid van de verspreiding van zorginnovaties te verklaren. In: Raad voor de Volksgezondheid en Zorg, ed. Weten wat we doen: verspreiding van innovaties in de zorg. Zoetermeer: Raad voor de Volksgezondheid en Zorg;, 2005; p. 7-54.

26. Schrijvers G, Oudendijk N, De Vries-Ek P, Hageman-Raatgever M, eds. Moderne patiëntenzorg in Nederland. Van kennis naar actie. Maarssen: Elsevier Gezondheidszorg, 2002.

27. Steckler A, Goodman RM, Kegler MC. Mobilizing organizations for health enhancement. In: Glanz K, Rimer BK, Lewis FM, eds. Health behavior and health education. 3rd ed. San Francisco, CA: Jossey-Bass, 2002; p. 335-60.

28. Jansen MWJ. Hartslag 2. Projectplan. Maastricht: GGD Zuid-Limburg, 2005.

29. Ruland E, Van Assema P, Ament A, Gorgels T, Van Ree J. Hartslag Limburg: integrale gezondheidsbevordering in buurten, gemeenten, bij huisartsen en in het ziekenhuis. Tijdschrift voor Gezondheidswetenschappen 2006;84:83-9. 
30. Rollnick S, Mason P, Butler C. Health behavior change. A guide for practitioners. Edinburgh: Churchill Livingstone, 1999.

31. Hart- en Vaat Centrum Maastricht. Visiedocument. Maastricht: azM, 2005.

32. Creer TL. Self-management of chronic illness. In: Boekaerts M, Pintrich PR, Zeidner M, eds. Self-regulation: theory, research, applications. San Diego, CA: Academic Press;, 2000; p. 601-6.

33. Groene $\mathrm{O}$, Garcia-Barbero M. Health promotion in hospital: evidence and quality management: World Health Organization Europe, 2005.

34. Groene O, Garcia-Barbero M. Health promotion in hospital: a strategy to improve quality in health care. European Journal of Public Health 2005;15:6-8.

35. Harting J, Van Assema P. Quality assessment of health counseling: performance of health advisors in cardiovascular prevention. Patient Education and Counseling 2004;54:107-18.

36. Harting J, Van Assema P, Ruland E, et al. Implementation of an innovative health service. A "real-world" diffusion study. American Journal of Preventive Medicine 2005;29(2):113-9.

37. Harting J, Van Assema P, De Vries NK. Patients' opinions on health counseling in the Hartslag Limburg cardiovascular prevention project: perceived quality, satisfaction, and normative concerns. Patient Education and Counseling 2006;61:142-51.

38. Ashenden R, Silagy C, Weeler D. A systematic review of the effectiveness of promoting lifestyle change in general practice. Family Practice 1997;14:160-75.

39. Bock BC, Carmona-Barros RE. Program participation and physical activity maintenance after cardiac rehabilitation. Behavior Modification 2003;27:37-53.

40. Bogers RP, Vijgen SMC, Bemelmans WJE. Costs of lifestyle interventions within health care and the amount of weight loss achieved. Bilthoven: RIVM, 2006.

41. Britt E, Hudson SM, Blampied NM. Motivational interviewing in health settings: a review. Patient Education and Counseling 2004;53:147-55.

42. Burke BL. The efficacy of motivational interviewing: a meta-analysis of controlled clinical trials. Journal of Consulting and Clinical Psychology 2003;71:843-61.

43. Cavill N, Buxton K, Bull F, Foster C. Promotion of physical activity among adults. Evidence into practice briefing. London: NHS National Institute for Health and Clinical Excellence, 2006.

44. Fokkema MR, Muskiet FAJ, Van Doormaal JJ. Leefstijlinterventie ter preventie van harten vaatziekten. Nederlands Tijdschrift voor Geneeskunde 2005;149:2607-12.

45. Harland J, White M. The Newcastle exercise project: a RCT of methods to promote physical activity in primary care. British Medical Journal 1999;319:828-32.

46. Harting J. Individual life style advice: development, implementation, and evaluation within the Hartslag Limburg cardiovascular prevention project [PhD thesis]. Maastricht: Maastricht University; 2005.

47. Harting J, Van Limpt P, Van Assema P, Van Ree J, De Vries NK. Proces- en effectevaluatie van het hoogrisicoproject binnen Hartslag Limburg. Tijdschrift Sociale Gezondheidszorg 2006;84:343-53.

48. Hillsdon M, Foster C, Cavill N, Crombie H, Naidoo B. The effectiveness of public health interventions for increasing physical activity among adults: a review of reviews. Evidence briefing. London: NHS Health Development Agency, 2005.

49. Mullen PD, Mains DA, Velze R. A meta-analysis of controlled trials of cardiac patient education. Patient Education and Counseling 1992;19:143-62.

50. Murchie P, Camell NC, Ritchie LD, Simpson JA, Thain J. Secondary prevention clinics for coronary heart disease: four year follow-up of a randomised controlled trial in primary care. British Medical Journal 2003;326:84-89.

51. NHS Centre for Reviews and Dissemination University of York. Cardiac rehabilitation. Effective Health Care 1998;4:1-12. 


\section{CHAPTER 5}

52. Proper KJ, Hildebrandt VH. Effect of individual counseling on physical activity fitness and health. American Journal of Preventive Medicine 2003;24:218-26.

53. Raftery JP, Yao GL, Murchie P, Campbell NC, Ritchie LD. Cost-effectiveness of nurse led secondary prevention clinics for coronary heart disease in primary care: follow-up of a randomised controlled trial. British Medical Journal 2005;330:707-10.

54. Riemsma RP, Pattenden J. Systematic review of the effectiveness of stage-based interventions to promote smoking cessation. British Medical Journal 2003;326:1175-7.

55. Unal B, Critchley JA, Capewell S. Modelling the decline in coronary heart disease deaths in England and Wales, 1981-2000: comparing contributions from primary prevention and secondary prevention. British Medical Journal 2005;331:614-9.

56. Scheirer MA. Is sustainability possible? A review and commentary on empirical studies of program sustainability. American Journal of Evaluation 2005;26:320-47.

57. Van Assema P, Mesters I, Kok G. Het focusgroep-interview: een stappenplan. Tijdschrift Sociale Gezondheidszorg 1992;70:431-7.

58. Sekhuis LM. Blijven bewegen, hard nodig. Behoud van lichamelijke activiteit bij Hartslag 2 patiënten. Maastricht: Universiteit Maastricht, 2006.

59. Cloosterman ML. Gedragseffecten van health counselling op korte en lange termijn. Een kwantitatief onderzoek naar de veranderingen in leefstijl bij patiënten met hart- en vaatziekten. Maastricht: Universiteit Maastricht, 2005.

60. Ebben N, Jansen MWJ. Resultaten van Hartslag 2. Eindrapportage Hartslag 2. Resultaten van een onderzoek naar koppeling van curatie en preventie door middel van leefstijladvisering. Maastricht: GGD Zuid-Limburg, 2006.

61. Gorissen P. Vascular medicine, putting people in the middle. Maastricht: Academisch Ziekenhuis Maastricht, 2001.

62. Vrijhoef HJM, Steuten LMG. Innovatieve zorgconcepten op een rij: disease management. Tijdschrift Sociale Gezondheidszorg 2005;83:305-6.

63. Eijkelberg IMJG, Mur-Veeman IM, Spreeuwenberg C, Koppers RLW. Patient focus groups about nurse-led shared care for the chronically ill. Patient Education and Counseling 2002;47:319-36.

64. Vrijhoef HJM, Diederiks JPM, Spreeuwenberg C. Effects on quality of care for patients with NIDDM or COPD when the specialized nurse has a central role: a literature review. Patient Education and Health Counseling 2000;41:243-50.

65. Dutch Heart Foundation, Revalidatiecommissie NHS/NVVC. Richtlijn Hartrevalidatie 2004. Den Haag: Nederlandse Hartstichting, 2004.

66. Kwaliteitsinstituut voor de Gezondheidszorg $\mathrm{CBO}$ en Nederlands Huisartsen Genootschap. Multidisciplinaire richtlijn Cardiovasculair Risicomanagement 2006. Alphen a/d Rijn: Van Zuiden Communications, 2006.

67. Ministerie van Volksgezondheid Welzijn en Sport. Preventienota Kiezen voor Gezond Leven. Den Haag: Ministerie VWS, 2006.

68. Grol R. Personal paper: beliefs and evidence in changing clinical practice. British Medical Journal 1997;315:418-21.

69. Grol R, Grimshaw J. From best evidence to best practice: effective implementation of change in patients' care. Lancet 2003:1225-30.

70. Berwick DM. Disseminating innovations in health care. JAMA 2003;289:1969-75.

71. Oldenburg BF, Hardcastle, Kok G. Diffusion of innovations. In: Glanz K, Rimer BK, Lewis FM, eds. Health behavior and health education. Theory, research and practice. 3rd ed. San Francisco, CA: Jossey-Bass, 2002; p. 312-34.

72. Riley BL. Dissemination of heart health promotion in the Ontario public health system: 1989-1999. Health Education Research 2003;18(1):15-31.

73. Yin RK. Case study research. design and methods. Beverly Hills: Sage Publications, 1994. 


\title{
CASE 6. OVERVIEW OF APPLIED PRACTICAL STRATEGIES
}

\author{
The administrative level
}

After an expert meeting in 1994, initiated by the mayor of one of the Southern Limburg municipalities, the Netherlands Heart Foundation concluded that the time had come for local policy makers, the GGD, GPs and hospitals to join forces to address the main risk factors for cardiovascular disease. The Foundation was prepared to give financial support, which stimulated the willingness among local authorities to participate and co-finance the project. The mayor, who was also a member of the Board of the Netherlands Heart Foundation, forcefully encouraged the initiative [advocacy +$]$ and the issue was put on the local political agenda. The agenda setting evolved into a window of opportunity as problems, policy and politics coincided [agenda setting +]. At the initiative of the local authorities, a coalition was built in the Southern Limburg region, consisting of representatives of the local authorities, the managing director of the GGD, professors at Maastricht University, the head of the local welfare agency and the Netherlands Heart Foundation. They formally declared their intention to contribute to the jointly formulated goals and preconditions [coalition building + ]. The functioning of a coalition is fostered when there is a common goal and enthusiasm, which in retrospect prevailed in 1998. However, it is hampered when persistent problems arise without positive prospects. After 2002, problems about the continuation of financial support dominated the agenda. Social entrepreneurs who were prepared to invest much of their time were no longer able to influence the attitude of policy makers. The project leader frequently consulted the various parties from the research, practice and policy domains to boost their willingness to collaborate and to continue the collaboration, which appeared to be crucial in 2002 [sidestepping the formal system +]. However, the collaboration process showed signs of weariness and a policy entrepreneur was lacking. Finally, the coalition collapsed.

The national government provided additional budgets for the large Dutch cities to stimulate infrastructure, economic and social investments in deprived areas, a policy programme known as the Large Cities Policy (Grote Steden Beleid). The Heartbeat framework design was approved at the meeting of the Municipal Council and financed under this policy programme, which focuses on reducing social inequalities. With the town of Maastricht functioning as the leading partner in public health, the other municipalities followed the Maastricht example, and resources were allocated [resource negotiations +]. The Large Cities Policy includes a list of proposals for temporary and permanent projects. In retrospect, the fact that the Heartbeat programme was put on the list of temporary projects allowed the project proposals to be redefined after 4 years, which in 2002 led to the discontinuation of the community part of 


\section{CHAPTER 5}

Heartbeat. The community part of the programme was formally accepted by the GGD Board as part of its Diversified Products Provision Model, but without financial compensation for the implementing agencies, making the decision an empty gesture.

\section{The institutional level}

Management support focused on the transition from the current state to the desired goals, which were expressed at the administrative level. The diagnosis regarding the urgency of the programme and the willingness to collaborate on the issue was part of the coalition building process, in which the institutional managers were involved as social entrepreneurs [diagnosis +]. The municipal authorities commissioned the GGD to prepare a comprehensive and integrated action plan. This plan was meticulously elaborated and involved contributions from the research, policy and practice domains, including activities that were proposed to promote collaboration [activities and interventions +]. Key players were identified and they jointly committed themselves to the mission and the goals, which was confirmed by contractual agreements [commitment +]. An intersectoral management structure was agreed upon. The complexity of this structure was simplified by creating different working groups at the community and municipal levels, involving general practitioners, specialists, health promoters, dieticians, welfare workers and researchers, all coordinated by the steering committee, consisting of managers, the mayor and aldermen, university professors and the project leader [intersectoral management structure + ]. A communication structure was designed to guide and plan the various activities that were to be undertaken to communicate the programme beyond the actual group of partners [communication structure +]. Evaluation of both the community and the high risk approach was part of the programme. During the implementation stage, the idea was launched to study the sustainability of the collaboration, and the project leader elaborated this idea into an evaluative research study on collaboration between the policy, practice and research domains [evaluation +$]^{334}$. Resources were made available in the form of various grants and support from the municipal administration. Institutional managers approved the estimated time and money investments, and they assigned staff to implement the collaboration project. However, the head of the Maastricht municipal Department of Education, Culture, Welfare and Sport did not manage to secure additional funds, which meant that the civil servants were basically given an extra task without being allocated the necessary facilities. This was not the case in the other municipalities, were civil servants were given extra time or task reduction [resources +- ].

The mission statement was frequently repeated in the communication messages. The organization of quarterly meetings appeared to be a successful 
strategy to connect institutions and to express the jointly perceived mission [mission statement + ]. The communication plan involved a quarterly newsletter, with media portrayals such as interviews with professionals, community members, patients and volunteers. The communication strategy strengthened a positive learning climate and a joint working culture. However, it proved difficult to find local policy makers prepared to act as role models [interviews with role models +-]. No internships were arranged [internships -]. Within and between the collaborating groups, the project leader stimulated a culture of rendering services (many activities relating to smoking, sports, exercise and nutrition were supplied by one group to another), sharing tools (e.g., software, materials, tailored food advice), and formal and informal consultations [rendering services, access to tools +], [formal and informal consultations +]. Regular diagnostic feedback to institutional staff was routine and part of the consultation strategy. For example, an application to extend the research period was made to the Netherlands Heart Foundation when the time schedule proved unfeasible [diagnosis and feedback + ]. Staff were recruited to do the additional work and agencies were contracted to render services. For instance, the welfare agency was asked to implement the community approach. The strategy to recruit extra staff was, however, not successful for the Maastricht policy domain [recruiting and retaining staff +- ].

The use of performance indicators and professional development policy was facilitated by the institutional managers from the research and practice domains, and resources for capacity enhancement were partly earmarked beforehand. For example, supervision of the health advisors (which were an important component of the programme) was organized, and a business methods training was offered by the National Institute of Health Promotion and Disease Prevention to support the 'healthy meat' initiative, in which private and public partners worked together. The policy domain did not participate in the professional development programmes [performance indicators and professional development +-$]$.

Kick-off conferences were organized in 1998 (for the Heartbeat Limburg programme) and in 2005 (for Heartbeat 2), and a few smaller and more informal gatherings were organized each year to build and maintain solidarity and unity. During a final conference on Heartbeat Limburg in 2005, the Netherlands Heart Foundation reviewed the pros and cons, facilitators and barriers, and derived national recommendations from them, from the research, practice and policy perspectives ${ }^{335}$ [kick-off conference + ]. The leader frequently had meetings with all of the partners to discuss the progress, the constraints and the benefits [sidestepping the formal system + ]. 


\section{CHAPTER 5}

\section{The individual level}

The prevailing situation in terms of individual competencies was diagnosed from the start of the project, and regular feedback to professionals was organized [diagnosis and feedback + ].

Arguments were solved by means of multidisciplinary group discussions to focus attitudes and norms on collaboration. Discussions on the programme content stimulated affective ties between the collaborating individuals and informal consultations. The working group consisting of researchers and practitioners proved a fruitful source of new ideas to improve the Heartbeat programme. Within the community working group, ideas evolved from the collaboration between practitioners and civil servants. Group discussions during formal and informal meetings were initiated by the project leader to influence the attitudes of GPs, cardiologists, vascular surgeons and internists [group discussion in multidisciplinary groups + ]. Professionals working in the community or with high-risk groups received instructions, after which they learned by doing. Community workers and volunteers were coached on activity planning and group discussions [learning by doing + ]. Detailed action plans, work books and materials were developed by the professionals to ensure efficient and correct implementation of the interventions [detailed action plan, formats, materials + ]. The health advisors and the members of the community working groups organized information exchange by peer networks [feedback by peer networking + ].

The prospects for future implementation were comprehensively debated, especially with civil servants [lectures and discussion +]. Certain parts of the programme were supported by master classes, such as a master class for the intervention on main meal quality and fruit intake among people with financial problems. The health advisors were trained in the technique of motivational interviewing, supported by research to assess the progress of their skills [master classes with skills training + ].

Publications and conference presentations outside people's own niche were encouraged and coached, as all partners were convinced of the importance of translating new knowledge into practice and policy, because of the growing need for evidence-based public health. More than fifty publications in various forms were produced. Research articles were published in Dutch scientific journals, reducing the language barrier, and in professional journals to reach different target groups [in-service coaching to publish beyond one's own niche $+]$. 


\section{Comments}

This case illustrates the complexity of and constraints on attempts to connect the policy domain with the practice and research domains. The implementation of a multi-factorial and multi-component cardiovascular risk factor intervention aimed at community and high-risk people was successful in terms of the creation of a coalition between various practitioners, researchers and policy makers. It was also successful in terms of the institutional link between the research and practice domains and the changes achieved within the organizations. However, the policy domain frequently failed to collaborate. For example, the policy makers did not accept the institutional consequences of their claim to operate as leaders of the intersectoral collaboration, as they were unable to facilitate the required civil servants. Essential preconditions at the institutional level of local government were not fulfilled, whereas the practice and research domains did succeed in this endeavour. The policy domain insufficiently participated in professional development policies, and failed to provide the required role models. The local authorities decided to terminate the financial support for the community part of the programme, despite the encouraging results reported by the research and practice domains. The underlying reasons for this decision are currently the subject of further research ${ }^{334}$. A provisional conclusion is that it was mainly due to lack of political expediency, cutbacks in the local public health budget and the lack of GGD leadership, the latter caused by external circumstances such as a merger. Whatever the reasons, the relation with local policy appeared to be complex and difficult all along the line, while the collaboration between the practice and research domains flourished. The interaction between practitioners and researchers was very productive in terms of mutual inspiration, combining theory and practice, and publishing results outside one's own niche. The disparities between the work cycles of practice and research were given conscious attention whereas the ones between policy on the one hand and practice and research on the other were not.

There were many interconnections between the policy, practice and research work cycles. Research work cycles were implemented regarding process factors, which were subsequently fed into the practice work cycle. For instance, within the community part of the programme, the practitioners went through the practice work cycle, followed by research on organizational involvement in health promotion activities relating to healthy eating, physical activity and smoking. This process evaluation measured change at the community level, which could be used in the policy work cycle to reconsider the impact of organizational involvement in the community ${ }^{329}$. The quality of health counselling by the health advisors was examined, followed by improvements to practice ${ }^{321}$. A randomized controlled trial was conducted to measure the effects of the highrisk approach and to translate the results into practice ${ }^{318}$. Research into ethical 


\section{CHAPTER 5}

aspects of health promotion practice yielded valuable recommendations for public health policy ${ }^{336}$. An overall effect evaluation was carried out to allow valid conclusions at the population level. The collaboration also contributed to evidence-based public health, since the Heartbeat programme was evaluated as an evidence-based intervention ${ }^{316}$. The total programme took the form of a helix, consisting of a complex set of interconnections between the research and practice work cycles, and to a lesser extent also with the policy work cycle. To attain policy decisions based on evidence appeared to be a rather complex phenomenon. Strikingly, the high-risk approach at the hospitals was the only segment of the original Heartbeat programme which was continued, even though it was evaluated as the least effective, relative to the high-risk approach in general practices and the community approach. The programme's continuation at the hospitals was facilitated by similarity in goals of the two collaborating institutions, both of which aim at improving health. The continuation of the health counselling component after 2006 will be enhanced by the use of new concepts that have evolved in the meantime, such as substitution of tasks.

To resume, this case study has revealed the strength - or should one say the weakness? - of evidence-based public health, which was judged differently by those who have the legitimate power to make policy. Policy making is not entirely a rational process, and this case study has highlighted the complexity and the barriers that exist especially in the policy domain. 


\section{GENERALIZING THE FINDINGS OF THE MULTIPLE-CASE STUDY}

Based on the findings of the multiple-case study, we can try to formulate more general propositions to bridge the gaps between the policy, practice and research domains. In this discussion, successful collaboration is defined as continued concerted action beyond the initial period. Five of the six projects studied above received some kind of grant during the initial phase, resulting in accountability to the grant providers in terms of achievements and expenditures, which necessitated partners to collaborate during the initial period. Continuing such collaboration beyond the initial project period, however, requires internal motivation on the part of the partners, rather than external obligation. Hence, we distinguished the cases that were successful and not successful in continued concerted action after the initial phase.

Below, we comment on the application of practical strategies in terms of the extent and quality and the effects in terms of evidence-based public health. The extent has been quantified as the number of practical strategies applied (see table 5.1, page 220-221). Their quality as such was not assessed quantitatively, and is described here in terms of general impressions or critical appraisal. The evidence base has been valued positively if it matched the definition of the World Health Organization 24,25 . The WHO defines evidence as: findings from research and other knowledge that may serve as a useful basis for decision making in public health and health care. The main aim of the evidence base is to help ensure that decision makers use the best available evidence.

\section{RESULTS}

The results of the multiple-case study allow the following conclusions to be drawn.

In terms of success, three of the six projects achieved collaboration beyond the initial period, viz. hepatitis B, Schoolbeat, and Heartbeat, with total scores (out of a maximum of 60 points) of 35, 53 and 54, respectively. Although the grants in these three cases were not entirely stopped, the budgets were substantially reduced after the initial phase. The checklist of 30 practical strategies allows a maximum score of 60 points. There seems to be a cut-off point for success, since three cases had high to very high scores, and three cases had substantially lower scores. A successful project would appear to require the application of a number and specific type of practical strategies at each level. 


\section{CHAPTER 5}

Table 5.1 Assessment of the implementation of practical strategies

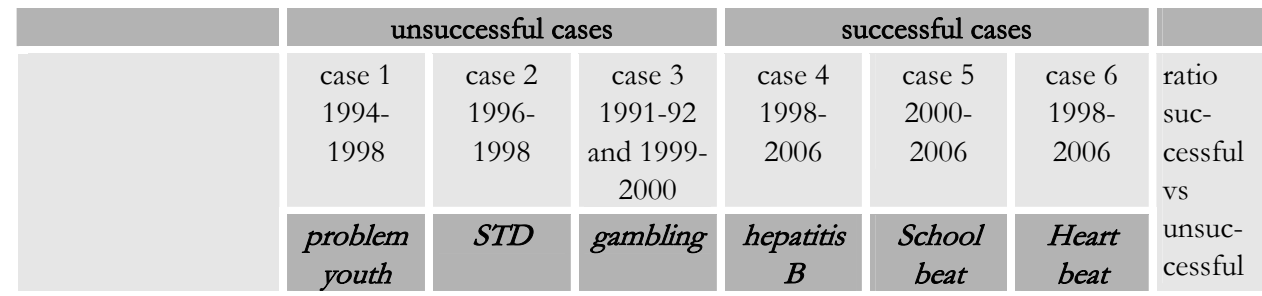

APPLICATION OF PRACTICAL STRATEGIES AT THE ADMINISTRATIVE LEVEL

\begin{tabular}{|c|c|c|c|c|c|c|c|}
\hline advocacy & + & - & - & - & + & + & $4 / 2$ \\
\hline coalition building & - & - & +- & - & - & + & $2 / 1$ \\
\hline $\begin{array}{l}\text { sidestepping the } \\
\text { formal system, } \\
\text { lobbying }\end{array}$ & - & - & +- & - & + & + & $4 / 1$ \\
\hline agenda setting & + & - & + & - & +- & + & $3 / 4$ \\
\hline $\begin{array}{l}\text { resource negotia- } \\
\text { tions }\end{array}$ & + & - & +- & -1 & + & + & $4 / 3$ \\
\hline subscore $(\max 10)$ & 6 & 0 & 5 & 0 & 7 & 10 & $17 / 11$ \\
\hline \multicolumn{8}{|c|}{ APPLICATION OF PRACTICAL STRATEGIES AT THE INSTITUTIONAL LEVEL } \\
\hline diagnosis of OD* & - & - & +- & + & +- & + & $5 / 1$ \\
\hline $\begin{array}{l}\text { action planning for } \\
\text { OD: } \\
\text { activities and inter- } \\
\text { ventions }\end{array}$ & - & - & - & + & +- & + & $5 / 0$ \\
\hline commitment & - & - & - & - & + & + & $4 / 0$ \\
\hline $\begin{array}{l}\text { intersectoral man- } \\
\text { agement structure }\end{array}$ & - & - & + & + & + & + & $6 / 2$ \\
\hline $\begin{array}{l}\text { communication } \\
\text { structure }\end{array}$ & +- & - & - & + & + & + & $6 / 1$ \\
\hline evaluation & - & - & - & + & + & + & $6 / 0$ \\
\hline resources & +- & - & + & + & + & +- & $5 / 3$ \\
\hline mission statement & - & - & - & - & +- & + & $3 / 0$ \\
\hline $\begin{array}{l}\text { interviews with role } \\
\text { models }\end{array}$ & - & - & - & + & + & +- & $5 / 0$ \\
\hline internships & +- & +- & +- & - & + & - & $2 / 3$ \\
\hline $\begin{array}{l}\text { rendering services } \\
\text { between niches; } \\
\text { access to tools of } \\
\text { other niches }\end{array}$ & +- & +- & + & + & + & + & $6 / 4$ \\
\hline $\begin{array}{l}\text { formal and informal } \\
\text { consultation struc- } \\
\text { tures }\end{array}$ & + & +- & + & + & + & + & $6 / 5$ \\
\hline
\end{tabular}


MULTIPLE CASE STUDY

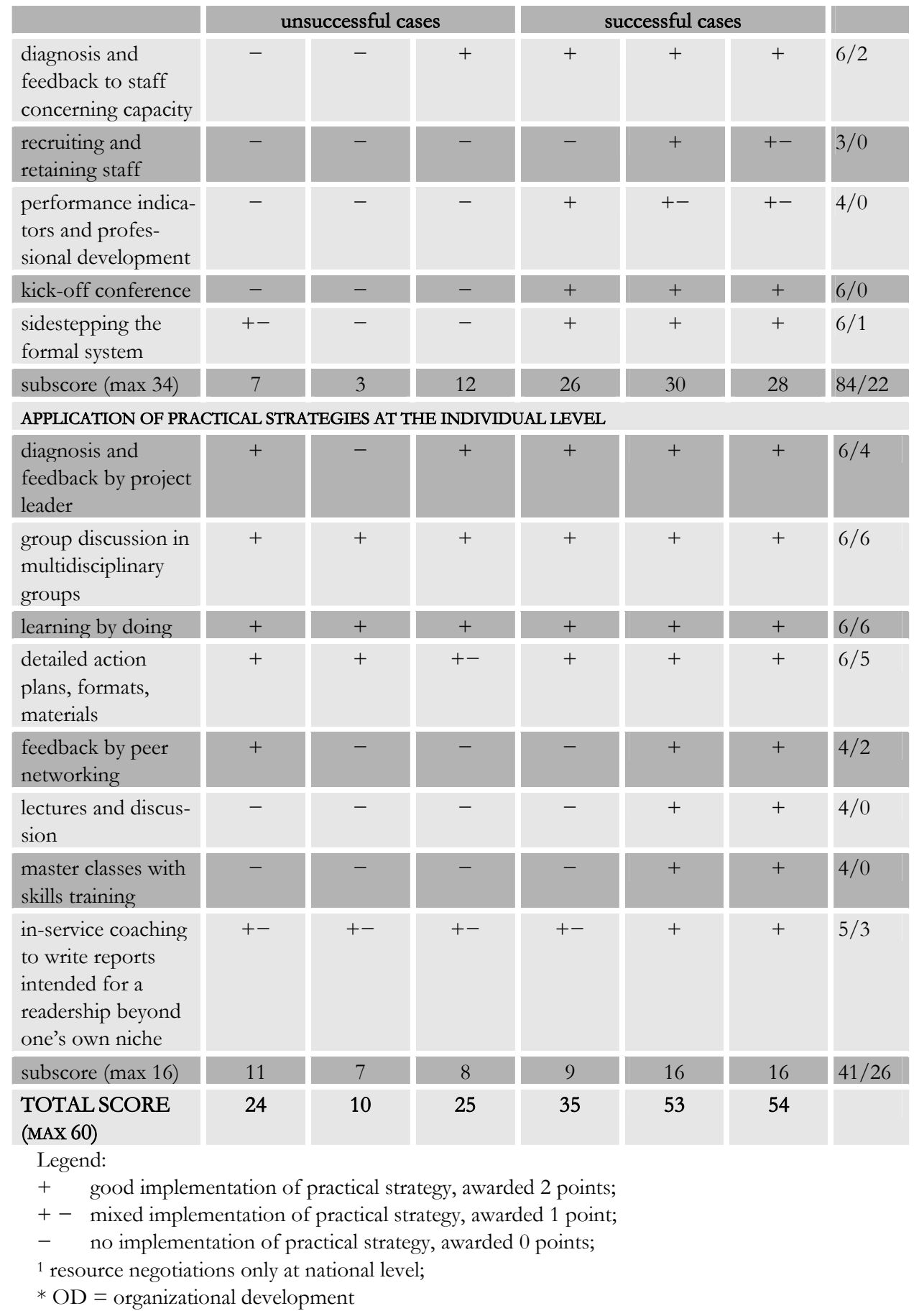




\section{CHAPTER 5}

\section{The administrative level}

Hepatitis B vaccination for high-risk groups was initiated by national policy makers, and the contribution of local policy makers was negligible. Consequently, the extent to which practical strategies were applied at the local administrative level was zero. The National Coordination Centre for Communicable Diseases Outbreak Management carried out the time-consuming negotiations at national policy level. This case illustrates the positive effect at the local level of national policies, with relevant expertise supplied by national institutions. $\mathrm{Na}$ tional policy makers may save local partners from time-consuming preparations at the administrative level.

The two other successful cases were not supported by national policy incentives; they were initiated locally. The successful Schoolbeat and Heartbeat projects implemented considerably more strategies at the highest system level than the STD project, but about the same as the problem youths and gambling projects. These findings seem to stress the importance of applying strategies at administrative level in locally developed projects. However, investing in practical strategies does not guarantee continuation, as was shown by the problem youths and gambling cases, where investments - in terms of checklist scores were equal to those for Schoolbeat.

On the other hand, not investing makes successful collaboration between research, practice and policy unlikely. Without horizontal connections at the administrative level between the actors of the policy, research and practice domains collaboration did not sustain. More than that, in the absence of any initial links at the administrative level between the policy domain on the one hand and the practice and research domains on the other, it will be difficult to feed the research findings into the policy domain afterwards, as was shown by the STD case. Such initial links seem to be a prerequisite to link work cycles from the practice, research and policy domains and to achieve effective public health policy.

Maximized application of practical strategies at the administrative level may predict but does not guarantee successful collaboration in the long run, as was shown by the case of the Heartbeat programme. The programme was successful in terms of continuation beyond the initial four years, but it was not successful in sustaining collaboration until it was routinized. As long as an innovation is not incorporated into the Diversified Products Provision Model, and thus considered a regular task of the GGD, it remains important to keep the administrative coalition together.

We did not measure the quality with which practical strategies were applied. Our impression is that those strategies which were used, were applied in a qualitatively acceptable manner, but we are aware that these were subjective observations. Research into the quality of partnerships and coalitions is gener- 
ally still subject to many limitations ${ }^{337}$. It is important to improve our ability to effectively measure the quality of the partnership process at the administrative level and the effects of quality differences. Measurement tools remain to be developed, which may be a subject for future research.

\section{The institutional level}

At institutional level, 17 practical strategies were proposed (implying a maximum of 34 points). The projects in the six cases had a mean score of 17.6, with a rather high deviation around the mean (17.6 \pm 10.3 points), indicating a high variance in total scores. Only high scores were associated with success in terms of sustained collaboration. This finding suggests a relatively strong contribution of organizational development strategies to successful collaboration.

A tendency of change over time was observed with regard to the amount of attention devoted to organizational development. Whereas in the first period of intended collaboration between the three domains (1991-1998) the focus was on practical strategies at the individual level, the focus shifted in the second period (1998-2006) as more emphasis was placed on organizational development (OD) strategies. Public health professionals, whether researchers, practitioners or policy makers, are generally not experts in organizational development. Practical strategies were applied without being consciously recognized as elements of organizational development. The term OD was never mentioned, nor were theory books referred to or OD experts consulted. However, strategies such as a diagnosis of the initial situation, activities and intervention planning related to collaboration, commitment strategy, intersectoral management structure, communication structure, evaluation, resource negotiations, role models, a diagnosis of staff capacity, recruitment of staff with specific competencies, performance indicators and professional development, a kick-off conference and leadership quality were more commonly applied after 2000 than before. These practical strategies were proposed to facilitate the conscious considerations of the differences between the work cycles and to upgrade competences to overcome these differences. If we make a distinction between successful and unsuccessful cases, it appears that it were exactly these strategies which contributed to success. The shift in strategies applied over time can be explained by the professional development of public health staff, who gradually learned from experience. Our study indicates that expertise in organizational development appears to be beneficial for sustained collaboration.

Local policy makers claim to be in charge of local public health, a view which is shared by the national government. The role of process manager requires certain facilitating conditions to be met at the institutional level, such as allocating civil servants to the task, and allocating sufficient time and mandate to supervise other agencies; participation in professional development pro- 


\section{CHAPTER 5}

grammes; consultations with agencies and feedback to all those involved. The role of process manager consists of creating horizontal links at the institutional level with the actors of the other domains. In two of the four cases in which the role of process manager was needed these preconditions were not adequately met.

As regards the quality of the strategies applied, it is important to devote attention to some critical limitations. On the whole, we doubt whether the practical strategies relating to organizational development (OD) were satisfactorily implemented. Often, too much attention was given to the content of the problem and not enough to the collaboration process itself. Practical strategies involving an entire organization were rarely if ever carried out. For instance, performance indicators and a professional development programme, which are formally the responsibility of the personnel department, were not given a high profile. Another limitation concerned the insufficient reciprocity of the relationships between the managers. For example, managers diagnosed staff capacity often restricted to the institute itself, and not extended to the capacity available beyond the own institute, which could have promoted collaboration. Strategies were more often applied vertically, i.e., support from the hierarchic manager to his personnel, rather than horizontally, i.e., reciprocity between the managers of the three domains.

\section{The individual level}

At the individual level, the average deviation from the mean was small (11.2 \pm 3.2 points). Success was not unequivocally predicted by scores on the strategy checklist. The Heartbeat (16 pts), Schoolbeat (16 pts) and hepatitis B (9 pts) projects were successful, whereas the problem youths project (11 pts) was not. This finding may have two implications, in that it may be assumed that individual strategies are insufficiently sensitive to predict successful collaboration, or that these strategies have no added value beyond a certain marginal value. The former interpretation implies a substantially greater influence on collaboration of strategies at the administrative and institutional levels, while the latter needs further research.

The implementation of practical strategies was perceived as rather easy compared to those at the institutional and administrative levels. The cases showed that when individual professionals from different institutions and disciplines were brought together to collaborate on a specific problem, they succeeded in coping with perceived barriers, reaching compromises in terms of content or process, keeping up dialogues, discussing matters, and finding ways of by-passing barriers when discrepancies could not be overcome. Perhaps the social commitment and passion for content issues of the people involved meant that they all aimed to contribute to solving the problem. The use of practical 
strategies in multidisciplinary groups stimulates people's performance, contributes to a feeling of ownership and counteracts resistance from others. Because an initiative to work beyond routine evokes more interest among innovators and early adopters than among the late majority of adopters and the laggards, the innovators and early adopters are overrepresented. In all cases, we experienced great enthusiasm, energy, willingness to be flexible and to take risks among the participating individuals. Based on these findings it seems that barriers to collaboration can be overcome by creating an atmosphere of learning from each other.

Just as at the administrative and institutional levels, the quality of the implementation of the practical strategies at the individual level was not measured. However, the use of these strategies did result in an interpersonal collaboration process in which the content of the problem played a dominant role. The professionals tended to make substantial contributions, as they sensed a reciprocal need for input from other domains in terms of the problem content. Our impression was that the quality of the implementation of these practical strategies was fairly good. Nevertheless, measurement tools need to be developed to assess quality and outcome.

\section{OVERALL CONCLUDING REMARKS}

The six cases unequivocally showed that collaboration cannot be sustained when institutional and administrative changes lag behind the individual changes. Whereas at the individual level, well-intentioned professionals may be successful in establishing collaboration, such undertakings are doomed to failure when institutional and administrative support is lacking. Only the successful cases, i.e., the hepatitis B, Schoolbeat and Heartbeat projects, were able to horizontally connect the actors at each system level and to vertically connect the actors of the three system levels, whereas the unsuccessful ones failed to do so. Administrative, institutional and individual changes keeping in step with each other seemed to be beneficial for successful collaboration beyond the initial period.

Successful collaboration implied repeated interconnections between the work cycles, as was shown in the successful cases, i.e., the hepatitis B, Schoolbeat and Heartbeat projects, whereas work cycles repetitions were absent in the unsuccessful cases, i.e. the problem youths, STD and gambling projects. We may conclude that there needs to be coherence between the system levels of the three niches. Coherence here is defined as vertical communication between the actors of the system levels, and horizontal communication between the actors of policy, practice and research at each system level and sometimes across the system levels. Communication between the system levels of the three 


\section{CHAPTER 5}

niches leads to a dialogue between the actors. Actors who discuss the results of the research work cycle may decide to enter these results into the practice and policy work cycle and vice versa. Therefore, discussion between the actors may improve the interconnections between the three work cycles. However, even such coherence did not guarantee success in the long run, as is shown by the case of the Heartbeat project.

In chronological terms, there was a shift from attention to individual strategies towards attention to institutional strategies, with the public health professionals obviously having learned from previous experience. Exactly these strategies contributed to success. The benefits of these learning processes should be disseminated within the field of public health to prevent others from making the same mistakes. These institutional and interinstitutional strategies were proposed to formally facilitate the conscious considerations of the disparities between the work cycles and to initiate a change process towards collaboration (see chapter 2). Obviously, such formalized opportunity to discuss the niche differences and to upgrade competences with respect to collaboration may prevent from conflicting orientations between practice, research and policy.

The quality of the application of OD-strategies was perceived as unsatisfactorily. Performance indicators and a professional development programme were given low profile. Experts judged the strategy of performance indicators as highly significant because the type of indicators represents an important link between the goal-setting process and the reward system.

We have introduced a framework of practical strategies at the various levels. We assessed the cases by assigning quantitative scores to the application of practical strategies. Merely assigning plus or minus scores to practical strategies is not likely to produce suggestions for their application in other public health cases. The application of practical strategies needs to be considered from the perspective of the particular context, historical roots, dynamics and unique persons. Application of a practical strategy in one case might be contraproductive in another case. In the Schoolbeat project, for example, it was consciously decided not to try and build a formal coalition, due to unfavourable administrative conditions at that moment. Similarly, the sum score at each level is not likely to predict success, as was shown by the successful case of hepatitis $\mathrm{B}$, in which the sum score at the administrative level was zero. Our method of assigning numerical scores to the six cases only served to visualize certain patterns from which lessons might be learned. Higher sum scores indicate multifactorial and multi-component strategies, and these are more likely to promote sustained collaboration, which corresponds to the existing knowledge about programme implementation.

We found that practical strategies at the administrative and institutional levels were harder to implement than those at the individual level, mainly due to 
the complexity and unpredictability of the contextual variables at the higher system levels. Besides, the time investments required at these levels were often perceived as 'not related to the core business'. The professionals involved might think it is not worth all their effort.

Although leadership qualities appeared important, the role and responsibilities of the project leader were not always clearly defined. Project leaders worked at the administrative, institutional and individual levels without formal responsibilities. A task division including responsibilities is needed between project leaders and institutional managers. The opportunity to contact stakeholders at higher system levels or across the niches should be anticipated by the formal management to support the project leader's goals.

The role of the local government in intersectoral collaboration programmes was not clearly formulated. Certain facilitating conditions from the local government, such as allocating civil servants to the task, consultations with agencies and feedback to all those involved, and allocating sufficient time and mandate were not adequately been met.

The differences in work pace between practice, policy and research were not consciously handled beforehand, apart from a few exceptions. None of the cases showed anticipated planning procedures to better fit the processes of the policy, practice and research work cycles. The word coincidence is most appropriate to describe the correspondence in pacing, if it occurred. Some pacing problems could not be solved, for example in the case of gambling policy. It was impossible to implement a research design that could lead to conclusive results on extended opening hours within gambling centres, given the time restraints applied by the city council. In case of the Schoolbeat project the pacing of the practice and research cycles diverged. The $\mathrm{PhD}$ dissertation of Schoolbeat could not be realized within the planned four year period due to the combination with the practice work cycle. In case of the Heartbeat project the policy process was not kept in pace with the needs of the practice cycle. A policy decision on the continuation of Heartbeat was postponed several times, which led to delay in the practical work. Harmonization of the time frames of the work cycles is strongly needed and requires much more attention of the professionals involved.

Our results regarding the assumption that collaboration contributes to evidence-based public health were not unequivocal and the assumption was not confirmed by the multiple-case study. The temporary collaboration established in the case of the problem youths project yielded evidence-based findings that were not translated into policy and practice. The STD case achieved neither the desired reliable and valid surveillance data nor the public health improvements in research, practice and policy. In the case of the gambling project, with its intermittent collaboration, the research domain was absent or entered into the collaboration only after the task had been assigned with policy restrictions. This 


\section{CHAPTER 5}

led to an inadequate research design without sophisticated evidence. In addition to these three unsuccessful cases, without effective contributions to evidencebased public health, there were three successful cases. The links between the practice, policy and research domains in the hepatitis B project yielded sustained collaboration and evidence-based practice and evidence-based policy regarding hepatitis $\mathrm{B}$ vaccination for high risk groups. In the case of the Schoolbeat project, the collaboration needs to be continued for a longer period to prove its potential effects in school health promotion, but preliminary results suggest positive effects. The Heartbeat project achieved a longstanding collaboration, in which positive health effects at population level were obtained through reciprocal relationships between the policy, practice and research domains, although these relationships could unfortunately not be sustained in the longer term. Research led to evidence-based practice which was not translated into evidence-based local policy.

A more exhaustive conclusion on the relation between establishing collaboration and producing useful evidence can only be drawn after further longitudinal research. 


\section{General discussion and future prospects}

\section{INTRODUCTION}

The main goal of this dissertation was to evaluate the potential for collaboration between the three component areas of pubic health: policy, practice and research. Collaboration will presumably result in the enhanced effectiveness, efficacy and efficiency of public health. This will result in higher quality standards when addressing public health problems. As discussed in chapter 2, we attribute the lack of interchange between the three public health domains to be due to the inherent characteristics of the niche domains. In the context of this dissertation, the term 'niche' refers to the specific ideologies, values, internal orientations, communication strategies, internal codes of behaviour, dynamics, and independence of each of the component areas of public health. The differences in work pacing is a problem that further complicates collaboration. The determinants of the barriers to collaboration between the niches were identified in chapter 3, which also proposed a number of practical strategies to modify these determinants. Experts from the three domains commented on the proposed practical strategies, resulting in some adjustments, as described in chapter 4. Our research hypothesis was that the application of multiple and various practical strategies would lead to sustained concerted action in the domains of policy, practice and research. In chapter 5 , six public health cases were used to evaluate the application of practical strategies and their contribution to collaboration. Based on this multiple-case study, we were able to draw conclusions about the potential of these practical strategies to overcome the barriers between the niches. This final chapter summarizes the main findings of our research and discusses both the cogency and the methodological quality of our study, after which we will reflect on the general conclusions and future prospects. 


\section{CHAPTER 6}

\section{GENERAL CONCLUSIONS}

The first research question we investigated was whether the inherent characteristics, within the domains of public health policy, practice and research, justified our proposition of the existence of niches. Our findings demonstrate that the domains of policy, practice and research differ in terms of frames of reference, time frames, working contexts, professional standards, goals and stakeholders. Each domain rests on underlying structures of rationality, perception and values, and can be regarded as a niche in which only the dynamics of that specific niche determine what actions are undertaken and how actions are evaluated. An analysis of the work cycles in the domains of policy, practice and research revealed thirteen major disparities between the niches. In themselves, there is nothing wrong with the frames of reference of the various niches, as these represent the trademarks of each profession. The problem is the inadequate interfaces between the frames of reference of the niches. The thirteen disparities we identified are frequently not given conscious attention and consideration when implementing public health policy. As shown by the six cases we evaluated, insufficient attention to these niche differences may result in controversy and conflicting orientations between policy makers, practitioners and researchers.

One important similarity between the work cycles was assessed. For each work cycle, recognition of a problem is the starting point for further action. Evidently, without a problem there is no reason to run the work cycle. This similarity, where the recognition of a problem instigates a work cycle, should be seen as an opportunity. The synthesis of the social, practical and scientific relevance of local public health problems has to be achieved in order to start collaboration.

Following this, a plausible, or - in Intervention Mapping terms - a logic model of the determinants of collaboration was constructed. Collaboration between the niches can be regarded as a change process that needs to be planned. The change process on which this dissertation focuses relates to local public policy. The process of policy making is a hierarchical process, with civil servants at the bottom, civil service managers in between, and the mayor, aldermen and municipal councillors at the top. The logic model distinguished the different system levels and the actors involved at each level, as they all play their own role in the change process. The application of Intervention Mapping resulted in a set of practical strategies that may be appropriate and effective in initiating and sustaining the change process that endeavours to improve collaboration between the niches. The application of the Intervention Mapping framework was found to be helpful and the protocol can obviously be applied beyond the issues for which it has been used so far, i.e., health promotion and energy saving. 
The Intervention Mapping approach was used to answer the third research question, namely how can the determinants of collaboration be influenced? Experts were asked to comment on theory-derived practical strategies, which resulted in a set of proposed practical strategies that were thought to support the collaboration process. This set of strategies should not be regarded as a general systematic roadmap from the present to the future for each collaboration process. An important aspect of Intervention Mapping is its tailoring, which implies that each problem, e.g., a collaboration process, needs to be studied as it is. Doing this may result in another set of practical strategies than the one described here.

Our final research question was: which practical strategies contribute to the development of collaboration between practice, police and research in local public health? In order to answer this question, we retrospectively evaluated the application of thirty practical strategies in six public health projects and the contribution of each to successes achieved in these projects. Successful collaboration was defined as concerted action sustained beyond the initial period of project-specific extra funding. The following practical strategies were evaluated at the three system levels:

The administrative level

- advocacy

- coalition building

- $\quad$ sidestepping the formal system, lobbying

- $\quad$ agenda setting

- resource negotiations

The institutional level, i.e., the formal organizations that facilitate or restrict actors in their activities

- diagnosis for organizational development

- action planning addressing organizational development: activities, commitment, intersectoral management structure, communication structure, evaluation, resources

- mission statement

- interviews with role models

- internships

- rendering services between the niches, access to tools in other niches

- formal and informal consultation structure

- diagnosis and feedback to staff concerning capacity

- recruiting and retaining staff

- performance indicators and professional development

- kick-off conference 


\section{CHAPTER 6}

- $\quad$ sidestepping the formal system, lobbying

The individual level

- $\quad$ diagnosis and feedback by project leader

- group discussions in multidisciplinary groups

- learning by doing

- detailed action plan, formats, materials

- feedback through peer networking

- lectures and discussions

- master classes with skills training

- in-service coaching to write reports or articles intended for a readership beyond one's own niche

Based on the study of the multiple cases, we were able to draw the following conclusions:

1. Sustained collaboration cannot be achieved when administrative and institutional changes lag behind the individual changes. Administrative, institutional and individual changes that keep pace with each other appear to be conducive to success. Although the changes at the local administrative level are less important in the case of national directives for local programmes, a coherent application of practical strategies seems to predict sustained collaboration in local public health. Successful collaboration between the niches can be achieved when various and multiple practical strategies are applied at each system level. The decisive number and specific type may differ in each case and may also depend on the context. We may conclude that coherence between the system levels of the three niches is necessary. Coherence is defined here as vertical communication between the actors of the system levels, and horizontal communication between the actors of policy, practice and research at each system level and sometimes across the system levels. Actors who discuss the results of the research work cycle may decide to enter these results into the practice and policy work cycles and vice versa. Discussion between the different actors may thus improve the interconnections between the three work cycles. However, our study also revealed that coherence does not guarantee sustained collaboration, as was shown by the case of the Heartbeat project. This may contradict the conclusion presented above. In short, coherence is necessary but not a guarantee.

2. The findings about practical strategies suggest that organizational development strategies that address collaboration at the institutional level make a relatively strong contribution. Organizational development $(\mathrm{OD})$ is a process that applies behavioural science knowledge to help organizations build the capacity to change and to achieve greater effectiveness. Organ- 
izational development strategies were selected to orient public health towards collaboration between its component areas of research, practice and policy. Our study revealed that only high overall scores for OD strategies were associated with success in terms of sustained collaboration. Organizational development strategies within and across niches appeared to be indispensable for the achievement of success. The tendency to change over time was observed with regard to the amount of attention devoted to organizational development. High scores were only achieved after 1998. Between 1991 and 2005, there was a shift in focus from individual strategies towards institutional strategies focusing on organizational development. The regional public health service (GGD) has obviously learned from previous experience. The benefits of these learning processes should be disseminated throughout the field of public health.

3. If collaboration between policy, practice and research is indeed valuable, the planned change process requires a clear task design. Although the local government is in charge of local public health, its role as a process manager in the collaboration process has not been realized. Partnerships represent a viable construction, but require initiative and subsequent process management with clear tasks and responsibilities. Our study revealed that there was a lack of clarity about who should assume the role of process manager at the institutional level: the civil servant who is in charge of public health, the civil service manager, or the GGD? In addition, the corresponding tasks and responsibilities were neither clearly defined nor allocated.

4. Performance indicators represent an important link between the goalsetting process and the reward system. Our study revealed that performance indicators combined with professional development to encourage professionals to collaborate with partners were undervalued by management. Strikingly, this was in direct contrast to the opinion of experts who judged that the strategy of using performance indicators was highly significant.

5. The application of the number and specific type of practical strategies depends on the particular context, historical roots, dynamics and unique persons involved. There is a need for a critical appraisal of strategies that can be meaningfully applied, given their effects on time and resource investment. Application of a particular strategy in one case might prove to be counterproductive in another case. The application of strategies other than those described here may be indicated when other determinants are identified in relation to a specific context. The context in which practical strategies are applied seems crucial to their ultimate effectiveness. For example, in the case of national initiatives for local public health programmes supported by expertise from national institutes, practical strate- 


\section{CHAPTER 6}

gies at the administrative level may be redundant and there may be fewer demands upon local leadership qualifications.

6. The differences in the pacing of work cycles between practice, policy and research were not anticipated. The case study revealed that none of the cases planned procedures beforehand to keep each process in pace with the needs of the other. The time frames of the work cycles need to be attuned to one another. Planning interconnections between the work cycles requires conscious prior planning by the professionals involved.

7. The assumption that successful collaboration between the three niches in terms of concerted action beyond the initial period of extra funding contributes to enhanced effectiveness, efficiency and efficacy of public health could not (or at least not yet) be unequivocally confirmed. The successful cases showed more work cycle repetitions, associated with an incremental evolution towards improved effectiveness, efficacy and efficiency. The successful concerted actions between research, practice and policy seem to indicate an evolution towards improved public health evidence.

\section{STRENGTHS AND LIMITATIONS OF THIS STUDY}

The research underlying this dissertation used a qualitative multiple-case study, a methodological decision which is open to debate. This section therefore discusses the strengths and weaknesses of the case study approach and also reflects on potential bias of the results.

The objective of study was to evaluate the degree of collaboration between the three domains of public health: policy, practice and research. A multiplecase study approach was considered to be the most appropriate means of investigating the research question which focused on "how" and "why" aspects within a real-life setting with researchers having little control over the events. The six cases included in this study are examples of research focusing on various types of public health problems. These cases represent a wide range of public health initiatives, including surveillance of infectious diseases, prevention of cardiovascular diseases, early detection of problem youth, school health promotion and gambling policy. Public mental health, which was not explicitly mentioned in this study, was integrated as part of both problem youth detection and the gambling policy projects. With the exception of environmental health and medical emergency care after accidents and disasters, the cases covered the essential public health functions as prescribed by the Dutch government. As well as reflecting a broad spectrum in terms of the content of the cases, the cases addressed different phases of the health promotion planning process. The problem youth detection case and STD case focused on epidemiological analy- 
sis. The gambling project studied the effects of environmental factors that may influence health. Hepatitis B and Schoolbeat stressed the need for a systematic intervention planning approach. Finally, Heartbeat examined the factors that are necessary for the sustainability of a successful intervention programme. Due to the diversity of the cases, we did not compare them, as this would have been unproductive. Rather, within the broad spectrum of cases, we compared the application of predefined practical strategies.

However, a case study method is characterized by certain weaknesses with regard to internal validity, external validity and reliability. Internal validity is defined as the soundness of the arguments and the line along which reasoning has developed ${ }^{137}$ or as the tenability of the relationship whereby certain conditions are shown to lead to other conditions ${ }^{132}$. To deal with concerns about internal validity, we first studied the niche characteristics within the areas of policy, practice and research and, in doing so, demonstrated the barriers to collaboration. Based on concepts of organizational development theory and health promotion theory, we developed a plausible, logic model of the determinants of collaboration. To change these determinants in order to prevent the barriers, practical strategies were proposed. The content of these strategies was operationalized on the basis of various theories relating to individual and organizational behavioural change. Eighteen highly trained and experienced professionals from the three domains were then asked to comment on the theorybased practical strategies that corresponded with the logic model. Based on the comments of the experts, the descriptions of the strategies were adjusted. This procedure resulted in agreement with the theory-based practical strategies and the experts' opinions. Thus, the plausibility of the logic model was confirmed by the experts. The application of the practical strategies was then evaluated by assessing six practical cases. Cases were described as intended collaborations between policy, practice and research. The case selection included only cases in which the author of this dissertation had participated in the 1991-2005 period. Making sound inferences, we linked the various theoretical concepts with practical strategies. Subsequently, we systematically collected data in order to analyse and interpret the results. This was accomplished by formulating strict definitions of the practical strategies, which were then also used as protocols for data collection. The application of practical strategies was documented for each individual case, enabling others to check the entire operationalization and thus enhancing the reliability and internal validity. Two other investigators independently used the protocol to assess the application of strategies in one specific case, and the results, in terms of reliability, were acceptable. Data were analysed by quantifying the number of strategies applied in each case. Since each case was studied separately, the results were interpreted against the background of case-relevant contextual characteristics, independent of the contexts of the other cases. Overall, the above approach strengthened the internal valid- 


\section{CHAPTER 6}

ity and reliability of our study and serious threats to the internal validity were eliminated.

External validity refers to whether a study's findings can be generalized beyond the immediate case study. One of the strengths of a case study design is the fact that the situation has not been created or manipulated by the investigator, which may enhance generalizability to real-life settings ${ }^{200}$. On the other hand, single cases tend to offer a poor basis for generalization. We used a multiple-case design because it can provide more compelling and robust evidence than a single-case design. Case studies rely on analytical generalization, which means that the investigator strives to generalize a particular set of results to develop some broader theory. On theoretical grounds, we hypothesized that a coherent application of theory- and expert-based practical strategies might reduce the barriers to collaboration. We found similar results in three cases. A small number of applied strategies unequally scattered across the three system levels did not lead to sustained collaboration. Our hypothesis was further corroborated by the results of three other cases, which demonstrated that a larger number of applied strategies, equally scattered across the system levels did result in sustained collaboration. On the continuum between absence and presence of sustained collaboration, we have obviously succeeded in selecting a range of cases varying from unsuccessful to successful, even though we cannot precisely define the two ends of the continuum. The fact that we found negative versus positive cases which could be reduced to the application of practical strategies strengthens the support for our hypothesis ${ }^{137}$ that a coherent application of practical strategies promotes sustained collaboration. However, the results of the multiple-case study should be interpreted cautiously and need to be substantiated with further empirical data.

The principal investigator in this research project had two roles. Between 1991 and 2005, she participated as a public health practitioner in each case, collaborating with researchers and policy makers. Thereafter, she started the research project and evaluated the collaboration process according to the protocol that she had developed together with experts. Data were collected retrospectively, starting in 2006. Since she did not function as an investigator during the implementation of the interventions of the case studies, there was no risk of unintentionally producing the expected results. On the other hand, the investigator implemented the actual inquiry alone, so subjectivity and induction on her part could be considered a threat to the validity of the research. For example, coming from the practice domain, she may have developed generalizations from specific observations within the field of policy. In the eyes of the investigator, the application of practical strategies in which policy makers were involved may be assessed differently than it would be when the strategies had been assessed by policy makers themselves. Although we did take preventive measures, as described above (intersubjective agreement on protocol; theory- 
based hypotheses), we must acknowledge that validity can be seriously endangered by bias wherein over-identification by the investigator with the object of study may occur. According to Maso \& Smaling ${ }^{137}$, methodological objectivity basically means doing justice to the object of study as it is perceived within the current context. From the viewpoint of the philosophy of science, Popper and Kuhn acknowledge that subjectivity in science is not only inevitable, but also necessary. Objective reality does not exist, as reality is always related to human discovery or observations ${ }^{130}$. The personal involvement of researchers is considered a valuable aspect as it stimulates rigorous research and offers access to other, unknown domains. Nevertheless, we tried to attain higher levels of methodological objectivity by consistently using theoretical concepts and protocols and by reflecting on contradictory findings.

Another aspect that may have threatened the objectivity of the research is the investigator's background. Since she works as a practitioner, she may have unconsciously valued the practical strategies derived from the practice perspective higher than those derived from the research or policy perspectives. On the other hand, she has collaborated extensively with researchers and policy makers for over eighteen years, thereby familiarizing herself with the research and policy perspectives. Nevertheless, we cannot exclude a possible bias in favour of the practice perspective.

Experimental designs require strict control over contextual factors, since changes in contextual factors are regarded as confounding. In qualitative and more exploratory designs, however, the contextual factors are used as important variables to improve our understanding of interactions within a particular social and cultural context. Additionally, evidence from experimental designs must prove its value in real-life situations involving different contexts. As part of the present analysis, we considered the contextual factors as important explanatory variables. For instance, in the case of the hepatitis B prevention project, the total number of practical strategies applied at the administrative level was zero, and the fact that it was nevertheless successful in terms of collaboration was explained by the condition of national rather than local policy decision-making. This context, where national policy is implemented locally, appeared to have a great impact on the final results in this case. This suggests that considering the context leads to a better understanding of the transferability of the evidence ${ }^{16,338,339}$.

Notwithstanding the progress we have made in this study, the results need to be substantiated with further empirical data. We suggest further research on the quality and intensity of the relationships between network actors and the effectiveness of networks with respect to collaboration. An instrument recently developed to diagnose the collaboration between partners of an intersectoral network is the Diagnosis of Sustainable Collaboration (DISC) model. The model focuses on opportunities and impediments for enhancing intersectoral 


\section{CHAPTER 6}

collaboration by assessing external factors, context, support, management and coordinated health promotion. DISC was applied to an intersectoral healthy school approach to test its usability. The results of the study reaffirm the importance of 'communication with policy-makers', 'adequate levels of contact among partners' and 'the need for consensus' 234 . Further research on network modelling might broaden and deepen insights into collaboration processes, and thereby add value to existing practices.

\section{DISCUSSION AND RECOMMENDATIONS}

\section{Conclusion 1: coherence in the application of practical strategies at the system levels of the niches seems beneficial to sustained collaboration}

The main conclusion of our research is that the application of practical strategies that vertically connect the actors of the three system levels and horizontally connect the actors of policy, practice and research is beneficial to sustained collaboration. Such coherence between the system levels of the three niches leads to a dialogue between the actors, which in turn may improve the interconnections between the three work cycles. The coherence corresponds to strategic, tactical and operational collaboration between the partners. However, a coherent application does not always guarantee sustained collaboration. The implications of this conclusion may be discouraging.

Although not all innovations can be sustained over long periods of time, in the case of a programme that is intended to be sustained after a trial, we recommend that the decision to start should be linked to the decision to continue (or at least the intent to continue). From the practice and policy perspective, implementing advocacy and building a coalition with the actors at the administrative level takes extensive preparation time. If these investments do not yield any long-term profit in terms of a reduction of public health problems, it would be better not to start. Moreover, it is ineffective, inefficient and frustrating to both professionals and the target population (and thereby irresponsible in times of scarcity) to spend considerable resources on the implementation of public health programmes that are discontinued soon after the initial funding ends. If continuation over time is necessary to achieve public health effects, then planning for sustainability is required. Compared to the current knowledge about sustainability, not much is known about proactive planning for sustainability. Knowledge about the determinants of sustainability is still at an early stage. Recently, attention to the sustainability of health promotion interventions is increasing globally. Categories of process indicators to help plan and monitor sustainability have emerged from empirical and theoretical research ${ }^{103,205,209,210 \text {, }}$ 340-344. We recommend using the available knowledge and selecting appropriate 
process indicators to plan for sustainability. Adequate process indicators may include the maintenance of health benefits, the level of routinization within the organizations, and measures of capacity building and resource availability. One might argue that this recommendation will block every local public health initiative. However, we recommend that the process should start with negotiations on the initiation and continuation of interventions, and the contributions to be made by all partners involved. Such negotiations should involve serious commitment by all parties, instead of the non-committal negotiations which have so often been conducted in the past. Evidently, a full guarantee of sustainability can neither be given nor be expected in advance. The actors at the administrative level have the formal power to continue or to stop collaborative initiatives related to local public health policy. We realize that a decision to initiate a nonrecurring programme (a one-off project) requires a different kind of preparation than letting stakeholders decide on a programme that requires prolonged and routinized implementation.

\section{Conclusion 2: organizational development strategies addressing collabo- ration within and across niches are indispensable to the achievement of success}

Coherence between the three system levels and the niches to initiate an organizational development process corresponds with theories and empirical data on organizational development and change management ${ }^{243,345-349}$. What we learn from our study is that there is a lack of competence among the managers in terms of applying strategies to address collaboration at the institutional level. While actors at the administrative level may agree that collaboration is a general goal, it are the actors at the institutional level who will have to facilitate the actual collaborative process, which is quite a different job. Their role is crucial, as concrete support from the management can be a decisive factor influencing the start, progress and outcomes of the change process. The efforts by management relate to a wider area than just that covered by the formal power they have. The practical strategies at this level need to be closely tailored to the culture and climate of the component public health organizations and must also be aimed at increasing capacity building. The multiple-case study discussed in this dissertation showed substantial failings in the use of practical strategies transcending an individual's own organization. Collaboration between individuals, representing organizations, can easily be confused with collaboration between organizations. However, the meaning of collaboration differs at each system level. Obviously, the GGD learned from previous experience, as well as the collaborating partners probably did. However, it is still an uncommon practice to think outside one's own organization and to orient oneself to improving the total public health system. Lack of time and priority setting to apply strategies 


\section{CHAPTER 6}

addressing organizational development may be serious constraints to improving the current situation. We recommend investing in time and priority setting for managers in the policy, practice and research domains. In particular, we should invest in goals and competences that should transcend their own niches. It is important to 'think outside the box' of public health and health promotion, and pay more attention to the bigger picture of organizational change processes to improve the quality of public health performance. Competence to apply OD strategies should consist of the four basic activities that managers need to jointly carry out during the change process. These basic activities should help them to (1) decide whether they want to engage further in a planned change programme and to commit resources to such a process; (2) diagnose the organizational problems and opportunities, including their causes and consequences; (3) design interventions to achieve the vision and goals of the collaborating organizations and to make an action plan for the implementation; and (4) evaluate the change process and provide feedback, rewards and training so the changes will persist.

\section{Conclusion 3: organizational development strategies addressing collabo- ration should clearly define and allocate the role of process manager}

Collaboration between policy makers, researchers and practitioners needs a formalized infrastructure. Organizing the implementation of practical strategies requires that professionals in the three domains specify roles, responsibilities, competences and authorizations. Various groups and activities need to be organized at the different system levels, including steering committees, manager meetings, project groups, informal discourse, etc. ${ }^{120,125,350}$. Our study revealed that not only responsibilities and competences but also mutual expectations were not always specified in advance. Prior specification might prevent disappointing contributions from one or more of the partners. In two of the six case studies, disappointments occurred due to unfulfilled process management roles on the part of local government that was expected by the practice domain. The changing roles of local authorities and the GGD, since the 2003 amendment to the Dutch Public Health Act (WCPV), have not yet resulted in a clear task division, especially for those involved at the institutional level. For example, it is still unclear who should assume the role of process manager in intersectoral collaboration programmes and what it exactly contains ${ }^{158}$. Policy makers and civil servants have many other issues to address and public health is often not a priority issue from a political perspective. In the Netherlands, the research domain of public health does have a tradition in research of management processes in intersectoral collaboration. However, the actual role of process manager does not belong to the core business of research organizations or universities. The GGD is the official implementation institute of local government with regard to public health. Coordination of intersectoral collaboration programmes 
should thus be a responsibility of the GGD. In the past, the public health sector has frequently taken the lead in public health changes. A leading role for professionals in the elaboration of public health plans fits into the current concepts about modernization of public administration and deregulation ${ }^{167}$. We recommend a leading role for the GGD as process manager of collaboration programmes. Research on the quality and effectiveness of the role of process manager should accompany the implementation.

\section{Conclusion 4: facilitating a planned change process towards collabora- tion requires additional performance indicators beyond one's own or- ganization}

Performance indicators are intended as tools for personal development ${ }^{351}$ as well as a means of ensuring accountability to management. The performance indicators developed so far have been related primarily to quantifiable results like the realization of client satisfaction, community participation, health promotion programmes, educational contributions, peer-reviewed publications and external funding. Performance indicators represent an important link between the goal-setting process and the reward system. Our study revealed that performance indicators related to collaboration were not given much attention by the management. Strikingly, this was in contrast to the opinion of experts who judged the strategy of performance indicators as highly significant. The challenge to collaborate may be a new institutional goal that should include an additional performance indicator. We recommend adding a new kind of accountability in public health, which is related to the collaborative process between policy makers, practitioners and researchers and which might be less quantifiable. In light of the current scarcity of resources, this recommendation may be met with resistance. Research organizations and universities are financed by the Ministry of Education, mainly based on such performance indicators as numbers of students, the ability to attract outside funding and number of dissertations. By contrast, the GGDs are financed by local governments and health insurance companies, based on performance indicators related to the so-called Diversified Products Provision Model. Local government budgets depend on local taxes, external funding and national incentives, and current performance indicators focus on quantified (financial) contributions to the municipal budgets and appropriate policy design. Neither the research domain, nor the practice domain and the policy domain are rewarded for engaging in collaborative efforts to upgrade public health quality. The challenge is thus to find performance indicators that yield mutual benefits.

Performance indicators can be formal or informal. People can be offered part-time positions within both the research and practice domains, giving them the opportunity to make use of all the university's research facilities and data 


\section{CHAPTER 6}

sources. The creation of these positions would result in win-win situations for the partners involved. Inviting university experts as honorary guests within the practice or policy domain could allow them to function as social entrepreneurs. Social entrepreneurs with a part-time position in the policy domain may stimulate the initiation of change processes. The goal is to find inducements that can attract the other partners. For example, contributions made by researchers to the evaluation of local public health policy may produce highly valued benefits in terms of policy and practice improvements. In this example, credit attributed to the research domain could be expressed in terms of publications, dissertations or a positive image resulting from the societal impact of the outcome. Additional to the citation impact factor, we suggest the societal impact factor of involving universities or research organizations as a relevant performance indicator. How to operationalize the idea of a societal impact factor needs to be further elaborated. To foster collaboration between the research, practice and policy domains, the selection of performance indicators should focus on encouraging educational efforts and mobilizing the professionals to work outside existing routines ${ }^{351}$. Kick-off conferences or short training sessions regarding the benefits of collaboration might be effective in generating enthusiasm among managers to introduce performance indicators addressing collaboration programmes. We recommend creating combined part-time positions within the research, policy and practice domains.

Organizations funding public health projects could impose specific performance indicators as an impetus for collaboration. The Netherlands Organisation for Health Research and Development (ZonMw) is an important funding organization for the healthcare and public health sectors, whose policy on grant allocation is mainly based on criteria related to health outcomes. Between 2001 and 2006, however, ZonMw's Healthy Living programme also awarded funding to project proposals addressing collaboration. Collaboration is a change process that aims to get various parties to work jointly on a specific problem. The Healthy Living programme assessed outcome criteria that were linked to the change process rather than to final health outcomes, although positive health outcomes are to be expected in the long term as a result of the collaboration process. The Healthy Living programme thus offered an opportunity to investigate collaboration structures. This specific ZonMw programme was terminated in 2005, without a follow-up. As a result, just as before 2001, the much-needed research on organizational development, change management, communication structures, network bounding, system modelling 352,353 and linkage systems once again has to compete for funding with epidemiological, medi$\mathrm{cal}$, clinical and health promotion research. More medically oriented research seems to be given priority in terms of fund allocation over research on organizational issues. Since our study revealed that the domains of practice, policy and research themselves are also inclined to focus on problem solving and 
achieving health outcomes, rather than on improving the organizational development towards collaboration, there is a need for an impetus towards collaboration programmes. The Implementation Research project within ZonMw's Prevention Programme offers the opportunity to incorporate the explicit goal of collaboration between the public health domains. Another possibility is to create a new ZonMw-programme on Public Health Services Research, in which collaboration can be addressed ${ }^{354}$. Since most contemporary public health problems are multi-factorial, no single organization can offer the full complement of information, resources, services and expertise necessary for population-wide improvement. In recognition of this, an additional goal for the ZonMw programme, addressing collaborative endeavours, would be highly justified.

\section{Conclusion 5: the application of the number and specific type of practi- cal strategies depends on the particular context}

As stated above, the set of proposed practical strategies should neither be regarded as a predictable systematic roadmap from the present to the future, nor as an overview of obligations of which at least half should be implemented. The set of practical strategies needs to be tailored to the context in which it is used. The selected practical strategies need to be organized into a programme that serves as a working tool during the collaboration process. There is one important premise underlying all practical strategies, which is 'reflection', as Schön \& Rein ${ }^{142}$ call it, or 'reframing' in the terminology used by Davis \& HowdenChapman $^{8}$. Controversies and conflicting orientations originate from differences in frames of reference between the three niches. These differences can be resolved when practitioners, researchers and policy makers are willing to reflect on their own frames of reference, or in other words, when they have a positive attitude towards transcending niche boundaries. Reflection on frames of reference can be used to design a mutually understood reality. Reframing should not be confused with problem solving, since problem solving is related to the content, not to the mode of thinking. Our study revealed that practitioners, policy makers, researchers and their managers are more inclined to think in terms of problem solving than in terms of reframing. What all practical strategies have in common is a face-to-face dialogue at the administrative, institutional and individual levels. A legitimate dialogue is the first step in the process of reframing and reflection. We recommend our set of practical strategies as a functional and evolving instrument that stimulates each partner to familiarize oneself with other frames of reference. The set of practical strategies, i.e., the collaboration programme, needs to correspond to the nature of the collaboration and the progress. 


\section{CHAPTER 6}

\section{Conclusion 6: the time frames of the work cycles can be better attuned to one another by paying attention to the possible interconnections well before implementation}

The time frames of the work cycles need to be attuned to one another to solve or reduce the problem of uneven pacing. Planning interconnections between the work cycles requires conscious consideration by the professionals involved. Within the policy domain, public health plans can be made in the first year of an alderman's four-year term of office, after which policy effects must be available in the fourth year. In the academic discourse, public health problems are generally studied in a context separate from the hectic atmosphere and pressure of the political arena. The practice domain is confronted with day-to-day problems and the continuous search for standards of practical utility as a part of public health policy. The actors in these three domains should make planning proposals to achieve consensus about a particular common public health problem and to link the work cycles that correspond with the political, research and practice time frames.

At the national level, when a new Minister of Public Health is appointed, the National Institute of Public Health and the Environment (RIVM) proposes to start a discussion with him or her about plans for the coming four years. Until now, it has not been customary to organize top-level consultations between the local policy, research and practice domains to deliberate on concerted public health activities for the next four years immediately after local elections. We recommend introducing such consultations between the alderman, the professor of public health and the GGD-director. These consultations may define the roles of the actors from the practice and research domains as social entrepreneurs, and that of the main actor from the policy domain as a policy entrepreneur ${ }^{112}$. At this level, a synthesis of social, practical and scientific relevance to local public health problems would then be achieved. For each work cycle, the recognition of a problem is the starting point for further action. Consensus about the first step of the work cycle, i.e. the problem, will result in running the work cycles simultaneously and allowing them to connect with each other when needed. An ongoing public health improvement plan could be established through a collaborative effort by local public health policy makers, managing directors and professors. The elected alderman could then use the plan as a monitoring tool to measure progress in public health and as means of ensuring accountability to the local government. 


\section{Conclusion 7: successful concerted actions by the research, practice and policy domains seem to be a starting point for the development of public health evidence}

The hepatitis B, Heartbeat and Schoolbeat projects discussed in this dissertation are examples of research findings being entered into the practice cycle. This process was assisted by further research. Real-life implementations resulted in policy applications, again assisted by further research. The work cycles of the research, practice and policy domains were repeated and linked, finally resulting in evidence that could be applied in practice and policy. The Heartbeat case, by contrast, failed in terms of evidence-based decision-making.

With respect to decision-making, the literature distinguishes between a technical, scientific rationality, which underlies research, and a politicaladministrative rationality, which underlies policy-making, reflecting an utopian versus a more pragmatic approach. This distinction is useful in addressing complex and dynamic decision-making processes ${ }^{88}$. In order to improve evidence-based public health, practitioners, policy makers and researchers need to jointly develop the technical and scientific evidence, and subsequently jointly address the policy context and political rationality to promote those decisions that are supported by the evidence base. As technical and scientific evidence accumulates incrementally through links between the work cycles, a longer time frame is needed. Complex, multi-component and multi-sectoral programmes should ideally be developed and assessed over a time frame of 10 to 15 years. This should involve testing the validity of the theory that defines the programme against the evidence of implementation in practice and policy and a range of short-, medium- and long-term programme outcomes. The lack of conclusive effects found in our study in terms of evidence of collaboration between the practice, policy and research domains was mainly due to a lack of long-term follow-up. We recommend long-term agreements on collaboration, with intermittent evaluations of work cycle links, using clearly formulated process and outcome criteria, in order to develop potentially effective public health programmes that can be sustained within public health policy and practice. Such long-term collaboration would correspond with the gradual developments in evidence-based public health.

\section{FUTURE PROSPECTS}

In 2005, the Ministry of Health, Welfare and Sport allocated extra resources as an impetus for collaboration between public health policy, practice and research. Recently, nine collaborative centres were initiated in the Netherlands. These centres are called 'Academic Collaborative Centres for Public Health' (In 


\section{CHAPTER 6}

Dutch: Academische Werkplaats). One of these centres is that in Limburg, in which the local municipal councils (19 in all), Maastricht University with its University Hospital and the regional GGD will collaborate on public health. The founding of an Academic Collaborative Centre for Public Health implies a transformation in the relationships between the partners. The lessons that can be learned from the present thesis will be used in the coming transformative process. The Academic Collaborative Centre for Public Health gives us the opportunity to integrate the activities of the practice, policy and research domains to strengthen the local knowledge infrastructure on public health, to upgrade competences of policy makers and practitioners by training and postgraduate courses, to build a sustainable service delivery structure for public health, to set research questions based on real-life situations, and ultimately, to improve public health.

Developing the Academic Collaborative Centre for Public Health is an enormous challenge, given that the relationship between the policy, practice and research domains has never been an easy one. There are obviously potential obstacles to a 'happy' synergy between research, practice and policy. Yet, there is a great deal to be learned from the rich and detailed reflections provided in the literature on the role of the social sciences in policy making and the role of evidence-based medicine in clinical practice ${ }^{102,104,107,108,355,356}$. Social science in the 1980s and, a decade later, evidence-based medicine aimed to contribute a more scientific and systematic approach to the policy making process respectively to clinical practice. The best research evidence was meant to be applied to mainstream policy and clinical practice. Research and scientific teaching should not just be limited to the academic environment. Both started with an overwhelming increase in interest as was illustrated by a continuous expansion of the social and medical literature. Gradually the field garnered its share of criticism. The criticism focused mainly on the methodological and ethical limitations of social science and evidence-based medicine. Problems appeared related to directly applying social research findings to policy and medical research findings to clinical practice. The rise and decline in social science and, to a much lesser extent, evidence-based medicine might also occur in the field of evidence-based public health. Evidence-based public health aims to contribute to a more scientific and systematic approach to public health policy and practice. While acknowledging many parallels with social science and evidencebased medicine, we observed that evidence-based public health might have some advantages. Research of Davis and Howden-Chapman ${ }^{8}$ has identified a number of factors associated with the successful translation of research into policy and practice that correspond with our research findings. The issue should be of local significance and proposed by the local authorities. Direct involvement of decision-makers was found to be an important indicator of success, as was clarity about roles in the project management. In general, pro- 
jects that were proximate to local policy were positively valued, while purely research exercises were not. Another factor that contributed to success was the application of appropriate methods by experienced investigators and their work with local data in collaboration with practitioners and policy makers, in a supportive environment with realistic time frames and sufficient resources. The above success factors need be taken into account in developing the Academic Collaborative Centre for Public Health. The synthesis of social, scientific and practical relevance towards solving a local public health problem should be the starting point for collaboration and should contribute to evidence-based local policy. However, the success of the collaboration between the practice, policy and research domains will be rather unpredictable and probably always depend on a melange of historical, organizational, cultural, political and human aspects. The challenge is to make the relationship work.

In view of the increasing pressure of public health problems on social equity, health inequalities and, ultimately, on today's health care system ${ }^{357,358}$, the Academic Collaborative Centre for Public Health should initiate new research, practice and policy methods to counteract the current trends. This thesis has also commented on further research activities. It will be necessary to develop instruments to monitor the collaboration process, measure the quality of practical strategies, and estimate cost-effectiveness. Furthermore, methods to improve the quality of data collection, strategies to optimize the translation of research findings into practice and policy, instruments to map environmental conditions, and strategies to translate local public health questions into research, must be created. The advantage of the Academic Collaborative Centre for Public Health will be that it allows for a much clearer linkage between local policy and local practice and research, based on local data. Joint problem selection and problem formulation may then foster the implementation of evidencebased public health practice and policy decisions that are based on solid evidence.

There are different levels of evidence, which can be ranked from low to high. The lowest level of evidence results from the opinion of one or more experts, while the next level is produced by non-experimental, descriptive research like comparative research, case studies and case-control designs. The following level of evidence is based on quasi-experimental, uncontrolled research. Subsequently, evidence from one randomized controlled trial (RCT) is then followed by evidence from more independent randomized controlled trials. The highest level of evidence stems from meta-analyses of trials. The evidence on public health programmes that results from RCTs almost never corresponds perfectly with the contextual environment in which it is to become implemented. This means that programmes have to be restructured and redefined to tailor them to contextual conditions. 


\section{CHAPTER 6}

A further distinction can be made between scientific evidence regarding effectiveness ('what works') and scientific evidence regarding context ('how or where it works' $)^{16,339,360}$. Context, however, is an elusive concept that needs to be defined by the most relevant dimensions related to the 'what' question. Even then, various dimensions may emerge. These dimensions include, but are not limited to, ethical, economic, implementation, organizational and attitudinal evidence. The existence of various dimensions may result in rather complicated and differentiated research studies that explore the evidence by triangulation. Achieving higher quality and more rigorous evidence on context is said to require a trans-disciplinary integration in public health research $206,217,262,352,360-362$. Trans-disciplinary integration strives 'to understand and reconcile linear and non-linear, qualitative and quantitative and reductionist and holist thinking and methods into a federation of approaches to system thinking and modelling' ${ }^{362}$. To find combinations of research methods that unravel the complexity of public health effectiveness is a challenge for the Academic Collaborative Centre for Public Health.

After the evidence regarding effectiveness and on context has been assessed, it is time to make decisions. There is no straightforward path between scientific evidence and policy decisions. To improve evidence-based decisionmaking, Lomas and colleagues ${ }^{16}$ advocated a so-called 'deliberative process', which has to be implemented according to a strict protocol. All background resources need to be available (i.e., all scientific research findings, well-defined questions to be answered), rules and expectations need to be clear, and participants should represent the research domain, the political domain, and the community, especially those people in the community who are expected to be affected by the programme. Evidence-based decision making is a provocative prospect for the Academic Collaborative Centre for Public Health.

Within the Academic Collaborative Centre for Public Health, various types of research may be meaningful. Whereas national institutes tend to engage in research activities like meta-analysis, research synthesis, theory-building research, national health surveys, molecular and genetic epidemiological studies, toxicological investigations and risk assessment, scenario research and dissemination research, the Academic Collaborative Centre for Public Health could concentrate on local public health issues. These could include local health surveys, research on policies that re-shape environmental conditions to improve health, and research on evidence-based decision-making in public health policy. Additionally, research on bringing about sustainable change through participation, research on intervention development, implementation research of evidence-based programmes, and studies that evaluate the effect of competence and skills training programmes could be examined. Finally, research on the organizational development of combinations of care with cure and prevention, and developing empowerment and ownership of change throughout a certain 
setting (e.g., schools, workplaces or communities), could be placed under the purview of the Academic Collaborative Centre for Public Health.

The Academic Collaborative Centre for Public Health will create opportunities to communicate across the niches. Communication is essential, as, in change processes, one component cannot change without modifications to the others. The partners in a dialogue can find mutually acceptable solutions through detection, interpretation, evaluation, recognition and interaction. $\mathrm{Mu}$ tual respect, ongoing commitment, trust and solidarity are essential elements needed to cut across values prevailing in the various niches and to initiate change that is supported by local politics. Communication within and across the niches is a suitable future prospect for the Academic Collaborative Centres for Public Health.

Lastly, it is our conviction that competent researchers, practitioners and policy makers can bridge the gaps between the niches in order to deliver higher levels of evidence for the field of public health. So far, practitioners were not encouraged to develop their hidden research or policy talents, as it did not belong to their core task. Mutatis mutandis, the same counts for researchers and policy makers. The collaboration within the Academic Collaborative Centre for Public Health may contribute to a further development of (hidden) talents of policy makers, researchers and practitioners. 


\section{References}

1. Van der Maas PJ, Mackenbach JP, eds. Volksgezondheid en gezondheidszorg. Maarssen: Elsevier/Bunge, 1998.

2. Detels R, Breslow L. Current scope and concerns in public health. In: Detels R, McEwen J, Beaglehole R, Tanaka H, eds. Oxford textbook of public health. Oxford: Oxford University Press, 2004: 3-20.

3. Tulchinsky TH, Varavikova EA. The new public health. An introduction for the 21 st century. San Diego: Academic Press, 2000.

4. Powles J. Public health policy in developed countries. In: Detels R, McEwen J, Beaglehole R, Tanaka H, eds. Oxford textbook of public health. Oxford: Oxford University Press, 2004: 263-280.

5. Bolton MJ, Stolcis BG. Ties that do not bind: musings on the specious relevance of academic research. Public Adm Rev 2003;63(5):626-630.

6. Byrne D. Enabling good health for all. A reflection process for a new EU health strategy. Brussels: European Commission, 2004.

7. Canadian Health Service Research Foundation. Weighing up the evidence. Making evidence-informed guidance accurate, achievable, and acceptable. Ottawa: Canadian Health Service Research Foundation, 2006.

8. Davis P, Howden-Chapman P. Translating research findings into health policy. $S_{c c} S_{C i}$ Med 1996;43(5):865-72.

9. Elliott H, Popay J. How are policy makers using evidence? Models of research utilisation and local NHS policy making. J Epidemiol Community Health 2000;54(6):461-8.

10. Erickson DL. The public health statute modernization national collaborative: developing a model state public health law. J Public Health Manag Pract 2002;8(1):39-46.

11. Frenk J. Balancing relevance and excellence: organizational responses to link research with decision making. Soc Sci Med 1992;35(11):1397-404.

12. Halverson PK. Embracing the strength of the public health system: why strong government public health agencies are vitally necessary but insufficient. J Public Health Manag Pract 2002;8(1):98-100.

13. Hasmiller S. Turning Point: The Robert Wood Johnson Foundation's effort to revitalize public health at the state level. J Public Health Manag Pract 2002;8(1):1-5.

14. Holland WW. Public health - its critical requirements In: Detels R, McEwen J, Beaglehole R, Tanaka H, eds. Oxford textbook of public health. Oxford: Oxford University Press, 2004: 1757-1764.

15. Kerkhoff AHM. Openbare Gezondheidszorg. Uitgangspunten voor een multidisciplinaire benadering. Budel: Damon BV, 2005.

16. Lomas J, Culyer T, McCutcheon C, McAuley L, Law S. Conceptualizing and combining evidence for health system guidance. Ottawa: Canadian Health Service Research Foundation, 2005.

17. Mackenbach JP. Sociale geneeskunde en 'public health': historische kanttekeningen bij de Nederlandse situatie. Tijdschrift voor Gezondheidswetenschappen 2003;81:450-458. 


\section{REFERENCES}

18. Mackenbach JP, Klazinga NS, van der Wal G. Preventie vraagt ambitieuzere anpak. Reactie op de kabinetsnota 'Langer gezond leven 2004-2007; ook een kwestie van gezond gedrag' [Prevention demands a more ambitious approach. Response to the cabinet policy document 'Live healthier for longer 2004-2007; also a matter of healthy behavior']. Ned Tijdschr Geneeskd 2004;148(15):704-7.

19. Nicola RM, Berkowitz B, Lafronza V. A turning point for public health. J Public Health Manag Pract 2002;8(1):iv-vii.

20. Nutbeam D. Getting 'evidence' into public health policy, and 'policy' into public health research. Tijdschrift voor Gezondheidswetenschappen 2003;81(3):155-158.

21. Post D, Groothoff W, eds. Sociale geneeskunde of public health. Toekomstperspectief van een uitdagend vakgebied. Houten/Mechelen: Bohn Stafleu Van Loghum, 2003.

22. Rychetnik L, Wise M. Advocating evidence-based health promotion: reflections and a way forward. Health Promot Int 2004;19(2):247-57.

23. Sabol B. Innovations in collaboration for the public's health through the Turning Point Initiative: the W.K. Kellogg Foundation perspective. J Public Health Manag Pract 2002;8(1):6-12.

24. World Health Organization. Handbook for evidence-based working and case study writing. Copenhagen: WHO Programme on Evidence on Health Needs and Interventions, 2006.

25. World Health Organization. Evidence policy for the WHO Regional Office for Europe. Copenhagen: WHO, 2004.

26. Berridge V, Stanton J. Science and policy: historical insights. Soc Sci Med 1999;49(9):11331138.

27. Beyer JM, Harrison TM. The utilization process: a conceptual framework and synthesis of empirical findings. Adm Sci Q 1982;27(4):591-622.

28. Raad voor Gezondheidsonderzoek. Advies kennisinfrastructuur public health: kennisverwerving en kennistoepassing. Den Haag: RGO, 2003.

29. Reijmerink W, Ravensbergen J. Haal meer uit kennis! Synthese van een veranderingstraject. Tijdschrift voor Gezondheidswetenschappen 2005;83:239-241.

30. Davis P. Problems, politics and processes: public health sciences and policy in developed countries. In: Detels R, McEwen J, Beaglehole R, Tanaka H, eds. Oxford textbook of public health. Oxford: Oxford University Press, 2004: 937-950.

31. Hamlin C. The history and development of public health in developed countries. In: Detels R, McEwen J, Beaglehole R, Tanaka H, eds. Oxford textbook of public health. Oxford: Oxford University Press, 2004: 21-38.

32. Doll R, Hill AB. Smoking and carcinoma of the lung; preliminary report. Br Med J 1950;2(4682):739-48.

33. Doll R, Hill AB. Mortality in relation to smoking: ten years' observations of British doctors. Br Med J 1964;1 (5395):1399-410.

34. Kickbusch I. The contribution of the World Health Organization to a new public health and health promotion. Am J Public Health 2003;93(3):383-8.

35. Baum F. The new public health. 2 ed. Melbourne: Oxford University Press, 2002.

36. Petersen A, Lupton D. The new public health. Health and self in the age of risk. London: Sage Publications, 1996.

37. World Health Organization. Ottawa Charter for Health Promotion. Ottawa: WHO, 1986.

38. Allwright S, Paul G, Greiner B, et al. Legislation for smoke-free workplaces and health of bar workers in Ireland: before and after study. Br Med J 2005;331(7525):1117.

39. Kickbusch I. The health society: importance of the new policy proposal by the EU Commission on health and consumer affairs. Health Promot Int 2005;20(2):101-3.

40. Merzel C, D'Afflitti J. Reconsidering community-based health promotion: promise, performance, and potential. Am J Public Health 2003;93(4):557-74. 
41. Schmid TL, Pratt M, Howze E. Policy as intervention: environmental and policy approaches to the prevention of cardiovascular disease. Am J Public Health 1995;85(9):120711.

42. Yancey AK, Lewis LB, Sloane DC, et al. Leading by example: a local health departmentcommunity collaboration to incorporate physical activity into organizational practice. $J$ Public Health Manag Pract 2004;10(2):116-23.

43. Commers MJ, Gottlieb N, Kok G. How to change environmental conditions for health. Health Promot Int 2007;22(1):80-87.

44. De Leeuw E. Health policy. An exploratory inquiry into the development of policy for the new public health in the Netherlands. PhD-thesis. Maastricht: Maastricht University, 1989.

45. Reid J. Choosing health? A consultation on action to improve people's health. London: Department of Health, 2004.

46. Von Schirnding Y. Health and sustainable development: can we rise to the challenge? Lancet 2002;360(9333):632-7.

47. Holland WW. Overview of policy and strategies. In: Detels R, McEwen J, Beaglehole R, Tanaka H, eds. Oxford textbook of public health Oxford: Oxford University Press, 2004: 257-261.

48. Signal L. The politics of health promotion: insights from political theory. Health Promot Int 1998;13(3):257-264.

49. Atwood K, Colditz GA, Kawachi I. From public health science to prevention policy: placing science in its social and political contexts. Am J Public Health 1997;87(10):1603-6.

50. Berwick DM. Disseminating innovations in health care. JAMA 2003;289(15):1969-75.

51. Bal R, Bijker WE, Hendriks R. Paradox van wetenschappelijk gezag. Over de maatschappelijke invloed van adviezen van de Gezondheidsraad. Den Haag: Gezondheidsraad, 2002.

52. Kohatsu ND, Robinson JG, Torner JC. Evidence-based public health: an evolving concept. Am J Prev Med 2004;27(5):417-21.

53. Potvin L, Haddad S, Frohlich KL. Beyond process and outcome evaluation: a comprehensive approach for evaluating health promotion programmes. In: Rootman I, Goodstadt M, Hyndman B, et al., eds. Evaluation in health promotion. Principles and perspectives. WHO Regional Publications, European Series no. 92. Copenhagen: WHO Regional Office for Europe, 2001: 45-62.

54. Chopra M, Darnton-Hill I. Tobacco and obesity epidemics: not so different after all? $B r$ Med J 2004;328(7455):1558-60.

55. Fleck F. Top health officials adopt global plan to cut obesity. Br Med J 2004;328(7451):1278.

56. World Health Organization. Global strategy on diet, physical activity and health. 57th World Health Assembly. Geneva: WHO, 2004.

57. Berridge V. Passive smoking and its pre-history in Britain: policy speaks to science? $S_{o c} S_{C i}$ Med 1999;49(9):1183-95.

58. Projectgroep Fusie GGD Zuid Limburg. Openbare Gezondheidszorg. Begripsbepaling en uitdagingen voor de regio Zuid-Limburg. Fusiebeslisdocument deel 1. Gemeenten Zuid Limburg: GGD Zuid Limburg, 2005.

59. Projectgroep Fusie GGD Zuid Limburg. Missie, visie en strategische doelen voor de regio Zuid-Limburg. Fusiebeslisdocument deel 2. Gemeenten Zuid Limburg: GGD Zuid Limburg, 2005.

60. Projectgroep Fusie GGD Zuid Limburg. Uitgangspunten en samenvatting productenboek. Fusiebeslisdocument deel 3, Bijlage Productencatalogus en Wetteksten. Gemeenten Zuid Limburg: GGD Zuid Limburg, 2005.

61. Platform Openbare Gezondheidszorg. Spelen op de winst, een visie op de openbare gezondheidszorg. Den Haag: Platform Openbare Gezondheidszorg, 2000. 


\section{REFERENCES}

62. Ministerie van Binnenlandse Zaken en Koninkrijksrelaties, Ministerie van VWS, Vereniging van Nederlandse Gemeenten, GGD Nederland. Nationaal Contract Openbare Gezondheidszorg. Utrecht: GGD Nederland, 2001.

63. Ministry of Health Welfare \& Sport (VWS). Living longer in good health. Also a question of healthy lifestyle. Netherlands Health-Care Prevention Policy. International Publication Series Health, Welfare and Sport no. 19. The Hague: Ministry of Health Welfare \& Sport, 2004.

64. Jansen J, Schuit AJ, Van der Lucht F. Tijd voor gezond gedrag. Bevordering van gezond gedrag bij specifieke groepen. Bilthoven: RIVM, Centrum voor Volksgezondheid Toekomst Verkenningen, 2002.

65. Ministry of Health Welfare \& Sport (VWS). How do we do? Health in the EU from a Dutch perspective; on the occasion of the Netherlands' EU Presidency 1 July-31 December 2004. The Hague: Ministry of Health, Welfare and Sport, 2004.

66. Raad voor de Volksgezondheid en Zorg. Het preventieconcert. Signalement over internationale vergelijking van publieke gezondheid. Zoetermeer: Raad voor de Volksgezondheid en Zorg, 2005.

67. Rijksinstituut voor Volksgezondheid en Milieu. Volksgezondheid Toekomst verkenning 1997. Bilthoven: Elsevier/De Tijdstroom, 1997.

68. Sociaal Cultureel Planbureau. Nederland in Europa. Sociaal en Cultureel Rapport 2000. Hoofdstuk 8: Gezondheid en Zorg. Den Haag: SCP, 2000.

69. Van der Lucht F, Verkleij H. Gezondheid in de grote steden. Achterstanden en kansen. Bilthoven/Houten: RIVM, Centrum voor Volksgezondheid Toekomst Verkenningen/ Bohn Stafleu Van Loghum, 2001.

70. Van Oers JAM, ed. Gezondheid op koers? Volksgezondheid Toekomst Verkenning 2002. Bilthoven/Houten: RIVM/Bohn Stafleu Van Loghum, 2002.

71. Visser M, Pluijm SM, van der Horst MH, Poppelaars JL, Deeg DJ. Leefstijl van 65-64jarige Nederlanders in 2002/'03 minder gezond dan in 1992/'93 [Lifestyle of Dutch people aged 55-64 years less healthy in 2002/'03 than in 1992/'93]. Ned Tijdschr Geneeskd 2005;149(53):2973-8.

72. World Health Organization. The world health report 2002. Reducing risks, promoting healthy life. Geneva: WHO, 2002.

73. World Health Organization. The European health report 2002. WHO Regional Publications European series. Copenhagen: WHO Regional Office for Europe, 2002.

74. GGD Nederland, NIGZ, VNG. Landelijk Convenant Gezondheidsbevordering in lokaal perspectief. Arnhem/Papendal, 2005.

75. Vereniging van Nederlandse Gemeenten. Brief aan de leden. Stand van zaken openbare gezondheidszorg. Den Haag: VNG, 2003.

76. Kornalijnslijper N, Schoenmakers C, Smeets K. Onderzoek gemeentelijke nota's gezondheidsbeleid. Den Haag: SGBO Onderzoeks- en Adviesbureau van de Vereniging van Nederlandse Gemeenten, 2005.

77. Inspectie voor de Gezondheidszorg [The Dutch Health Care Inspectorate]. Openbare gezondheidszorg: hoe houden we het volk gezond? Den Haag: Inspectie voor de Gezondheidszorg, 2005.

78. Hoogervorst JF. Brief van de minister van VWS aan de voorzitter van de Tweede Kamer der Staten-Generaal, kenmerk PG/OGZ 2.627.342, naar aanleiding van het rapport "Staat van de Gezondheidszorg 2005: Openbare Gezondheidszorg" van de Inspectie voor de Gezondheidszorg (IGZ). Den Haag: Ministerie van VWS, 2005: 1-7.

79. Tweede Kamer der Staten-Generaal. Rijksbegroting 2000. Voorstel van wet. Vaststelling van de begrotingsstaten van het Ministerie van VWS voor het jaar 2006. Den Haag: Ministerie van VWS, 2005. 
80. Raad voor de Volksgezondheid en Zorg. Gezond zonder zorg. Zoetermeer: Raad voor de Volksgezondheid en Zorg, 2000.

81. Joffe M, Mindell J. A framework for the evidence base to support Health Impact Assessment. J Epidemiol Community Health 2002;56(2):132-8.

82. Joffe M, Sutcliffe J. Developing policies for a healthy environment. Health Promot Int 1997;12(2):169-173.

83. Kemm J. Health impact assessment: a tool for healthy public policy. Health Promot Int 2001;16(1):79-85.

84. Macintyre S, Chalmers I, Horton R, Smith R. Using evidence to inform health policy: case study. Br Med J 2001;322(7280):222-5.

85. Putters K. HIA, the next step: Defining models and roles. Environmental Impact Assessment Review 2005;25(7-8):693-701.

86. World Health Organization. Health impact assessment: from theory to practice. Report on the Leo Kaprio Workshop 1999. Göteborg: The Nordic School of Public Health, 2000.

87. Bekker MPM, Putters K, Van der Grinten TED. Exploring the relation between evidence and decision-making: A political-administrative approach to health impact assessment. Environmental Impact Assessment Review 2004;24(2):139-149.

88. Bekker MPM, Putters K, van der Grinten TED. Evaluating the impact of HIA on urban reconstruction decision-making. Who manages whose risks? Environmental Impact Assessment Review 2005;25(7-8):758-771.

89. Kuiper W. Brief aan de Vaste Commissie voor Volksgezondheid Welzijn en Sport. Den Haag: VNG, 2006.

90. Evers IMH, Ubachs PJH. Voorkomen is beter dan genezen: 75 jaar georganiseerde gezondheidszorg te Maastricht. Maastricht: Stichting Historische Reeks Maastricht, 1996.

91. Houwaart ES. De hygiënisten. Artsen, staat en volksgezondheid in Nederland 1840-1890. Maastricht: Rijksuniversiteit Limburg, 1991.

92. Meijer AWM. Plaats en functie van de Nederlandse GGD-en. In: De Leeuw E, ed. Gezonde steden. Lokale gezondheidsbevordering in theorie, politiek en praktijk. Assen/Maastricht: Van Gorcum, 1991: 109-131.

93. Projectgroep Fusie GGD Zuid Limburg. Organisatieontwerp GGD Zuid Limburg. Fusiebeslisdocument deel 4. Gemeenten Zuid Limburg: GGD Zuid Limburg, 2005.

94. Projectgroep Fusie GGD Zuid Limburg. Gemeenschappelijke Regeling. Bijlage behorende bij fusiebeslisdocument deel 4. Gemeenten Zuid Limburg: GGD Zuid Limburg, 2005.

95. Inspectie voor de Gezondheidszorg [The Dutch Health Care Inspectorate]. Gezondheidsvoorlichting en -opvoeding bij GGD's. Rapport van een inspectieonderzoek. Den Haag: IGZ, 2000.

96. Van den Bosch N, Koornstra A, Mascini M. Gezondheidsbevordering bij GGD'en: document over de kern van de functie, kernaanbod en deskundigheidseisen. Utrecht: Nederlandse Vereniging voor Preventie en Gezondheidsbevordering / GGD Nederland, 2006.

97. GGD Nederland. Resultaten GGD Benchmark begroting 2003. Inzet van middelen en prestaties in beeld. Utrecht: GGD Nederland, 2004.

98. Projectgroep Fusie GGD Zuid Limburg. Financiën en begroting GGD Zuid Limburg. Fusiebeslisdocument deel 5. Gemeenten Zuid Limburg: GGD Zuid Limburg, 2005.

99. Raad voor Gezondheidsonderzoek. Advies Werkplaatsfunctie buiten het academisch ziekenhuis. Den Haag: RGO, 2000.

100. Mackenbach JP. Paradigma, succes en toekomst van de maatschappelijke gezondheidszorg. Tijdschrift voor Gezondheidswetenschappen 2001;79:522-529.

101. Dodds JM, Calleson DC, Eng E, Margolis L, Moore K. Structure and culture of schools of public health to support academic public health practice. $J$ Public Health Manag Pract 2003;9(6):504-512. 


\section{REFERENCES}

102. Gottlieb NH, Huang PP, Blozis SA, Guo JL, Murphy Smith M. The impact of Put Prevention into Practice on selected clinical preventive services in five Texas sites. Am J Prev Med 2001;21(1):35-40.

103. Grol R, Grimshaw J. From best evidence to best practice: effective implementation of change in patients' care. Lancet 2003;362(9391):1225-30.

104. Murphy-Smith M, Meyer B, Hitt J, Taylor-Seehafer MA, Tyler DO. Put Prevention into Practice implementation model: translating practice into theory. J Public Health Manag Pract 2004;10(2):109-15.

105. Nutbeam D. Getting evidence into policy and practice to address health inequalities. Health Promot Int 2004;19(2):137-40.

106. Steuten LMG, Van Merode GG, Spreeuwenberg C, Vrijhoef HJM. Brug tussen onderzoek en beleid: toepassing van health technology assessment op disease management. Tijdschrift voor Gezondheidswetenschappen 2004;82:118-119.

107. Taylor-Seehafer MA, Tyler DO, Murphy-Smith M, Hitt J, Meyer B. Ensuring quality preventive care: the Texas Department of Health's Put Prevention into Practice initiative. $J$ Public Health Manag Pract 2004;10(2):94-9.

108. Tyler DO, Taylor-Seehafer MA, Murphy-Smith M. Utilizing "PPIP Texas style!" in a medically underserved population. J Public Health Manag Pract 2004;10(2):100-8.

109. Tijssen IMJG. Kwaliteit noodt tot meer gebruik. Bruikbaarheid van sociaalwetenschappelijke onderzoeksresultaten volgens arts-bestuurders. Nijmegen: Katholieke Universiteit Nijmegen, 1988.

110. Schaap L, Van Twist M. Een consequent constructivistische kijk op geslotenheid in beleidsnetwerken. In: Abma T, In 't Veld R, eds. Handboek beleidswetenschap. Perspectieven, thema's, praktijkvoorbeelden. Meppel: Boom, 2001: 249-260.

111. Weiss CH. The many meanings of research utilization. Public Adm Rev 1979;39:426-431.

112. Hoeijmakers M. Local health policy development processes. Health promotion and network perspectives on local health policy-making in the Netherlands. Maastricht: Maastricht University, 2005.

113. Spreeuwenberg C. 'Kennisinfrastructuur Public Health'; een advies van de Raad voor Gezondheidsonderzoek ['Knowledge Infrastructure for Public Health': an advisory report from the Advisory Council on Health Research]. Ned Tijdschr Geneeskd 2003;147(50):2459-62.

114. Botvin GJ. Advancing prevention science and practice: challenges, critical issues, and future directions. Prev Sci 2004;5(1):69-72.

115. Dean K, Hunter D. New directions for health: towards a knowledge base for public health action. Soc Sci Med 1996;42(5):745-50.

116. Donker M. Torn between two lovers. Lokaal volksgezondheidsbeleid tussen politiek en wetenschap. Oratie. Rotterdam: Erasmus Medical Centre, 2006.

117. Dusenbury L, Hansen WB. Pursuing the course from research to practice. Prev $S_{C i}$ 2004;5(1):55-9.

118. Garretsen H. Zorgen om de zorg. Oratie. Tilburg: Universiteit van Tilburg / TRANZO, 2000.

119. Goodwin N. Leadership and the UK health service. Health Policy 2000;51(1):49-60.

120. Kimbrell JD, Witmer A, Flaherty P. The Louisiana Public Health Institute: a cross-sector approach for improving the public's health. J Public Health Manag Pract 2002;8(1):68-74.

121. Klazinga N. Ambachtelijk veranderen. In: Ravensbergen J, Friele R, Keijsers J, Wensing M, Klazinga N, eds. In zicht. Nieuwe wegen voor implementatie. Assen: Van Gorcum, 2003: 130-140.

122. Sturmans F. De GGD als academische werkplaats voor de maatschappelijke gezondheidszorg. Tijdschrift voor Gezondheidswetenschappen 1999;77:65-66. 
123. Van der Velden J. De toekomst van de Public Health. Oratie. Nijmegen: Radboud Universiteit, 2004.

124. Van Oers JAM. Voorkomen kan beter. Op zoek naar nieuwe wegen in de openbare gezondheidszorg. Oratie. Tilburg/Bilthoven: Universiteit van Tilburg/RIVM, 2004.

125. Wellcome Trust. Public health sciences: challenges and opportunities. Report of the Public Health Sciences Working Group convened by the Wellcome Trust. London: Wellcome Trust, 2004.

126. ZonMw. Aan tafel! Programma Academische Werkplaatsen Publieke Gezondheid 20052008. Den Haag: ZonMw, 2005.

127. Hoogerwerf A. Beleid, processen en effecten. In: Hoogerwerf A, Herweijer M, eds. Overheidsbeleid. Een inleiding in de beleidswetenschap. Alphen aan den Rijn: Samson, 1998: $17-36$.

128. Van Dijk J. Gemeentelijk gezondheidsbeleid. Omvang en doelgerichtheid. Groningen: Rijksuniversiteit Groningen, 2001.

129. Walt G. Health Policy. An introduction to process and power. London: Zed Books, 2004.

130. Chalmers A. Wat heet wetenschap. Amsterdam: Boom, 2003.

131. Pröpper I, Steenbeek D. De aanpak van interactief beleid: elke situatie is anders. Bussum: Coutinho, 1999.

132. Yin RK. Case study research. Design and methods. Beverly Hills: Sage Publications, 1994.

133. Polit DF, Hungler BP. Nursing Research. Principles and methods. Philadelphia: J.B. Lippincott Company, 1991.

134. Robson C. Designing case studies. In: Robson C, ed. Real world research. A resource for social scientists and practitioner-researchers. Oxford: Blackwell Publishers, 1993: 146-186.

135. Miles MB, Huberman AM. Qualitative data analyses. An expanded sourcebook. Thousands Oaks: Sage Publications, 1994.

136. Bouter LM, Van Dongen MCJM, Zielhuis GA. Epidemiologisch onderzoek. Opzet en interpretatie. Houten: Bohn Stafleu van Loghum, 2005.

137. Maso I, Smaling A. Kwalitatief onderzoek: praktijk en theorie. Amsterdam: Boom, 1998.

138. Abma T, In 't Veld R, eds. Handboek beleidswetenschap. Perspectieven, thema's, praktijkvoorbeelden. Meppel: Boom, 2001.

139. Keijsers J, Paulussen T, Peters L, Fleuren M, Lammers F. Kennis beter benutten: informatiegedrag van nationale beleidsmakers. Woerden: NIGZ en TNO, 2005.

140. Lindblom CE, Woodhouse EJ. The policy-making process. Englewood Cliffs, NJ: Prentice-Hall Inc., 1993.

141. Etzioni A. Mixed-scanning: a third approach to decision-making. Public Adm Rev 1967:385-392.

142. Schön DA, Rein M. Frame reflection. Toward the resolution of intractable policy controversies. New York: Basic Books, 1994.

143. Blum HL. Planning for health. Development and application of social change theory. New York: Human Sciences Press, 1974.

144. Stone D. Policy Paradox. The art of political decision making. New York: Norton \& Company, 2002.

145. Kingdon JW. Agendas, alternatives and public policies. New York: Addison-Wesley Educational Publishers Inc., 2003.

146. De Vries MS. Problemen op de agenda. In: Hoogerwerf A, Herweijer M, eds. Overheidsbeleid. Een inleiding in de beleidswetenschap. Alphen aan den Rijn: Samson, 1998: 39-57.

147. Rütten A, Von Lengerke T, Abel T, et al. Policy, competence and participation: empirical evidence for a multilevel health promotion model. Health Promot Int 2000;15(1):35-47.

148. Shiell A. Health outcomes are about choices and values: an economic perspective on the health outcomes movement. Health Policy 1997;39(1):5-15. 


\section{REFERENCES}

149. Van den Heuvel H. Beleidsinstrumenten. Naar strategisch beleid in netwerken. In: Abma T, In 't Veld R, eds. Handboek beleidswetenschap. Perspectieven, thema's, praktijkvoorbeelden. Meppel: Boom, 2001: 281-291.

150. Ministerie van Binnenlandse Zaken en Koninkrijksrelaties. Spelen met doorwerking. Over de uitwerking van doorwerking van de adviezen van adviescolleges in het Nederlandse openbaar bestuur. Tilburg/Utrecht: Universiteit van Tilburg/Berenschot, 2004.

151. Geul A. Beleidsconstructie, coproductie en communicatie. Zes beproefde methodieken van beleidsontwikkeling. Utrecht: Lemma BV, 1998.

152. Goumans M. Innovations in a fuzzy domain. Healthy cities and (health) policy development in the Netherlands and the United Kingdom. PhD-thesis. Maastricht: Maastricht University, 1998.

153. Herweijer M, Coolsma JC. Beleidsbepaling. In: Hoogerwerf A, Herweijer M, eds. Overheidsbeleid. Een inleiding in de beleidswetenschap. Alphen aan den Rijn: Samson, 1998: 79-97.

154. Hunter DJ. Public health policy. Oxford: Polity Press/Blackwell Publishing, 2003.

155. Maarse JAM. De uitvoering van overheidsbeleid. In: Hoogerwerf A, Herweijer M, eds. Overheidsbeleid. Een inleiding in de beleidswetenschap. Alphen aan den Rijn: Samson, 1998: 99-117.

156. De Bruyn H, Ten Heuvelhof E. Procesmanagement. In: Abma T, In 't Veld R, eds. Handboek beleidswetenschap. Perspectieven, thema's, praktijkvoorbeelden. Meppel: Boom, 2001: 193-207.

157. Putters K, Van der Grinten T. Schuivende institutionele verhoudingen in de besturing van de Nederlandse gezondheidszorg. In: Abma T, In 't Veld R, eds. Handboek beleidswetenschap. Perspectieven, thema's, praktijkvoorbeelden. Meppel: Boom, 2001: 111-126.

158. Rutgers ED, ed. Vormgeven aan maatschappelijke ondersteuning van jeugdigen. Onderzoek naar regie en samenwerking: over vertrouwen, wederkerigheid en zingeving. Den Haag: SDU, 2006.

159. Milio N. Promotion health through public policy. Ottawa: Canadian Public Health Association, 1989.

160. Milio N. Evaluation of health promotion policies: tracking a moving target. In: Rootman I, Goodstadt M, Hyndman B, et al., eds. Evaluation in health promotion. Principles and perspectives. WHO Regional Publications, European Series no. 92. Copenhagen: WHO Regional Office for Europe, 2001: 365-386.

161. Rütten A. Evaluating health public policies in community and regional context. In: Rootman I, Goodstadt M, Hyndman B, et al., eds. Evaluation in health promotion. Principles and perspectives. WHO Regional Publications, European Series no. 92. Copenhagen: WHO Regional Office for Europe, 2001: 341-365.

162. Leeuw F. Evaluatie-onderzoek, auditing en controle in Nederland anno 2000. In: Abma T, In 't Veld R, eds. Handboek beleidswetenschap. Perspectieven, thema's, praktijkvoorbeelden. Meppel: Boom, 2001: 70-82.

163. Tweede Kamer der Staten-Generaal. Preventieve gezondheidszorg. 's Gravenhage: SDU Uitgevers, 2003.

164. Pröpper IMAM. Terugkoppeling: de vertaling van evaluatie naar beleid. In: Hoogerwerf A, Herweijer M, eds. Overheidsbeleid. Een inleiding in de beleidswetenschap. Alphen aan den Rijn: Samson, 1998: 141-162.

165. Van der Krogt TPWM, Mol NP. Beleid en beheer. In: Hoogerwerf A, Herweijer M, eds. Overheidsbeleid. Een inleiding in de beleidswetenschap. Alphen aan den Rijn: Samson, 1998: 333-354. 
166. Bressers JTHA. De evaluatie van beleid. In: Hoogerwerf A, Herweijer M, eds. Overheidsbeleid. Een inleiding in de beleidswetenschap. Alphen aan den Rijn: Samson, 1998: 119139.

167. Van den Brink G. Van waarheid naar veiligheid. Amsterdam: Sun, 2006.

168. Teisman G. Perspectieven op beleidsprocessen: over fasen-, stromen- en rondenmodellen. In: Abma T, In 't Veld R, eds. Handboek beleidswetenschap. Perspectieven, thema's, praktijkvoorbeelden. Meppel: Boom, 2001: 302-309.

169. Van Strien S. Praktijk als wetenschap, methodologie van het sociaal-wetenschappelijk handelen. Assen/Maastricht: Van Gorcum, 1986.

170. Wagenaar H. Beleid als fictie: praktijk en verhaal in het openbaar bestuur. In: Abma T, In 't Veld R, eds. Handboek beleidswetenschap. Perspectieven, thema's, praktijkvoorbeelden. Meppel: Boom, 2001: 321-332.

171. Kuyvenhoven MM. Quality of performance of general practitioners confronted with patients' non-specific complaints. PhD-thesis. Utrecht: Universiteit van Utrecht, 1988.

172. Schuil PB. Algemene begrippen. In: Schuil PB, Bolscher DJA, Brouwers-de Jong EA, et al., eds. Nederlands leerboek voor de jeugdgezondheidszorg. Maastricht/Assen: Van Gorcum, 1987: 18-57.

173. Stolper CF, Rutten ALB, Dinant GJ. Hoe verloopt het diagnostisch denken van de ervaren huisarts? Huisarts en Wetenschap 2005;48:16-19.

174. Kuyvenhoven MM, Spreeuwenberg C, Touw-Otten FW. Diagnostic styles of general practitioners confronted with ambiguous symptoms. An exploratory study. Scand J Prim Health Care 1989;7(1):43-8.

175. Gezondheidsraad [The Health Council of the Netherlands]. From implementation to learning: the importance of a two-way dialogue between practice and science in health care. Den Haag: Gezondheidsraad, 2000.

176. Brug J, Schaalma H, Kok G, Meertens RM, Van der Molen HT. Gezondheidsvoorlichting en gedragsverandering. Een planmatige aanpak. Assen: Van Gorcum, 2000.

177. Green LW, Kreuter MW. Health promotion planning: an educational and ecological approach. 3 ed. Mountain View, CA: Mayfield Publishing Company, 1999.

178. Bartholomew LK, Parcel GS, Kok G, Gottlieb NH, eds. Planning health promotion programs. An Intervention Mapping approach. 2 ed. San Francisco: Jossey-Bass, 2006.

179. Gorissen WHM. Kennis als hulpbron. Het gebruik van wetenschappelijke kennis bij beleidsvorming in de jeugdgezondheidszorg voor 4-19 jarigen. PhD-thesis. Utrecht: Universiteit Utrecht 2001.

180. Gabbay J, Le May A. Evidence based guidelines or collectively constructed "mindlines?" Ethnographic study of knowledge management in primary care. Br Med J, 2004;329(7473):1013.

181. Cuijpers P, De Graaf I, Bohlmeijer E. Adapting and disseminating effective public health interventions in another country: towards a systematic approach. Eur $J$ Public Health 2005;15(2):166-9.

182. St Leger L. Building and finding the new leaders in health promotion: where is the next wave of health promotion leaders and thinkers? Are they emerging from particular regions, and are they less than 40 years old? Health Promot Int 2001;16(4):301-3.

183. Rogers EM. Diffusion of innovations. New York: Free Press, 2003.

184. Goodstadt MS, Hyndman B, McQueen DV, Potvin L, Rootman I, Springett J. Evaluation in health promotion: synthesis and recommendations. In: Rootman I, Goodstadt M, Hyndman B, et al., eds. Evaluation in health promotion. Principles and perspectives. World Health Organization Regional Publications, European Series, no. 92. Copenhagen: WHO Regional Office for Europe, 2001: 7-38. 


\section{REFERENCES}

185. Lakhani A, Coles J, Eayres D, Spence C, Rachet B. Creative use of existing clinical and health outcomes data to assess NHS performance in England. Part 1: performance indicators closely linked to clinical care. Br Med J 2005;330(7506):1426-1431.

186. Lapré R, Van Montfort G, eds. Bedrijfseconomie van de gezondheidszorg. Maarssen: Elsevier/De Tijdstroom, 1999.

187. Rootman I, Goodstadt M, Potvin L, Springett J. A framework for health promotion evaluation. In: Rootman I, Goodstadt M, Hyndman B, et al., eds. Evaluation in health promotion. Principles and perspectives. WHO Regional Publications, European Series no. 92. Copenhagen: WHO Regional Office for Europe, 2001.

188. Kok H, Molleman G, Saan H, Ploeg M. Handboek Preffi 2.0: richtlijn voor effectieve gezondheidsbevordering en preventie. Woerden: NIGZ, 2005.

189. Saan H, De Haes W. Gezond effect bevorderen. Het organiseren van effectieve gezondheidsbevordering. Woerden: NIGZ, 2005.

190. Molleman G. Preffi 2.0: health promotion effect management instrument. Development, validity, reliability and usability. PhD-thesis. Maastricht: Universiteit Maastricht, 2005.

191. Van de Poel F. Wat laat het GVO/preventieveld ons zien? Overview of health promotion activities in the Netherlands. Woerden: NIGZ, 2003.

192. De Regt W, Van Splunteren P, eds. Beter zo! Praktijkgevallen van inventieve innovatie. Assen: Van Gorcum, 2001.

193. De Groot AD. Methodologie. Grondslagen van onderzoek en denken in de gedragswetenschappen. Den Haag: Mouton, 1981.

194. Hofstee WKB. De empirische discussie. Theorie van het sociaal-wetenschappelijk onderzoek. Meppel: Boom, 1980.

195. Lave CA, March JG. Modellen in de sociale wetenschappen, een inleiding. Alphen aan den Rijn: Samson, 1980.

196. Glanz K, Rimer BK, Lewis FM, eds. Health behavior and health education. Theory research and practice. 3 ed. San Francisco: Jossey-Bass, 2002.

197. Buunk AP, Veen P. Sociale psychologie. Praktijkproblemen, van probleem naar oplossing. Houten/Diegem: Bohn Stafleu Van Loghum, 1995.

198. Baarda DB, De Goede MPM. Basisboek methoden en technieken. Handleiding voor het opzetten en uitvoeren van onderzoek. Groningen/Houten: Wolters Noordhoff, 2001.

199. Swanborn PG. Basisboek sociaal onderzoek. Meppel: Boom, 1991.

200. Swanborn PG. Sociaal-wetenschappelijk onderzoek en de samenleving. In: Swanborn PG, ed. Methoden van sociaal-wetenschappelijk onderzoek. Meppel: Boom, 1994: 377-411.

201. 't Hart H, Van Dijk J, De Goede M, Jansen W, Teunissen J. Onderzoeksmethoden. Hoofdstukken 3 en 11. Meppel: Boom, 1996.

202. De Beaufort I. Ethiek en medische experimenten met mensen. Assen/Maastricht: Van Gorcum, 1985.

203. Juttmann RE, Klazinga NS, Mackenbach JP. Wetenschappelijke kwaliteit van gezondheidszorgonderzoek. Uitgangspunten en richtlijnen. Tijdschrift voor Gezondheidswetenschappen 2004;82:518-522.

204. Biglan A. Contextualism and the development of effective prevention practices. Prev $S_{C i}$ 2004;5(1):15-21.

205. Glasgow RE, Vogt TM, Boles SM. Evaluating the public health impact of health promotion interventions: the RE-AIM framework. Am J Public Health 1999;89(9):1322-7.

206. Learmonth AM. Utilizing research in practice and generating evidence from practice. Health Educ Res 2000;15(6):743-56.

207. Pentz MA. Form follows function: designs for prevention effectiveness and diffusion research. Prev Sci 2004;5(1):23-9. 
208. Ravensbergen J, Friele R, Keijsers J, Wensing M, Klazinga N, eds. In Zicht. Nieuwe wegen voor implementatie. Assen: Van Gorcum, 2003.

209. Glasgow RE. Evaluation of theory-based interventions. The RE-AIM model In: Glanz K, Rimer BK, Lewis FM, eds. Health behavior and health education. Theory, research and practice. 3 ed. San Francisco: Jossey-Bass, 2002: 530-544.

210. Oldenburg BF, Sallis JF, French ML, Owen N. Health promotion research and the diffusion and institutionalization of interventions. Health Educ Res 1999;14(1):121-30.

211. Fleuren MAH, Wiefferink CH, Paulussen TGW. Determinants of innovations within health care organizations: Literature review and Delphi-study. Int J Quality Health Care 2004;16:107-123.

212. Rothman KJ, Poole C. Science and policy making. Am J Public Health 1985;75(4):340-1.

213. Sackett DL, Rosenberg WM, Gray JA, Haynes RB, Richardson WS. Evidence based medicine: what it is and what it isn't. Br Med J1996;312(7023):71-2.

214. Lockwood S. "Evidence of me" in evidence based medicine? Br Med J 2004;329(7473): 1033-5.

215. Muir Gray JA. Evidence based policy making. Br Med J 2004;329(7473):988-9.

216. Reilly BM. The essence of EBM. Br Med J 2004;329(7473):991-2.

217. Rimer BK, Glanz DK, Rasband G. Searching for evidence about health education and health behavior interventions. Health Educ Behav 2001;28(2):231-48.

218. Smith GD, Ebrahim S, Frankel S. How policy informs the evidence. Br Med J 2001;322:184-85.

219. Straus SE, Jones G. What has evidence based medicine done for us? Br Med J 2004;329(7473):987-8.

220. Weed DL. Towards a philosophy of public health. J Epidemiol Community Health 1999;53(2):99-104.

221. Wilson T, Holt T, Greenhalgh T. Complexity science: complexity and clinical care. Br Med J2001;323(7314):685-8.

222. Steenbakkers M, Bastiaens C, Leurs M, Ruland E, Jansen M. Vijf jaar community-based werken in Hartslag Limburg (1998-2003). Ervaringen uit de praktijk. Tijdschrift voor Gezondheidswetenschappen 2005;83(2):108-112.

223. Korsten AFA. Beleidsbeëindiging. In: Hoogerwerf A, Herweijer M, eds. Overheidsbeleid. Een inleiding in de beleidswetenschap. Alphen aan den Rijn: Samson, 1998: 163-181.

224. Klijn EH, Koppenjan J. Besluitvorming en management in netwerken: een multi-actor perspectief op sturing. In: Abma T, In 't Veld R, eds. Handboek beleidswetenschap. Perspectieven, thema's, praktijkvoorbeelden. Meppel: Boom, 2001: 179-195.

225. Lomas J. Using 'linkage and exchange' to move research into policy at a Canadian foundation. Health Aff (Millwood) 2000;19(3):236-40.

226. Lomas J. Health services research. Br Med J 2003;327(7427):1301-2.

227. Schrijvers AJP. Tien theorieën om de snelheid van de verspreiding van zorginnovaties te verklaren. In: Raad voor de Volksgezondheid en Zorg, ed. Weten wat we doen: verspreiding van innovaties in de zorg. Zoetermeer: RVZ, 2005: 7-54.

228. Van Splunteren P, Van Everdingen J, Janssen J, Minkman M, Van der Rouppe-Voort M, Schouten L. Doorbreken met resultaten. Assen: Van Gorcum, 2003.

229. Mintzberg H. Mintzberg over management. De wereld van onze organisaties. Amsterdam/Antwerpen: Contact, 1995.

230. O'Neill M, Lemieux V, Groleau G, Frotin F, Lamarche P. Coalition theory as a framework for understanding and implementing intersectoral health-related interventions. Health Promot Int 1997;12(1):79-87.

231. Provan KG, Veazie MA, Staten LK, Teufel-Shone NI. The use of network analysis to strengthen community partnerships. Public Adm Rev 2005;65(5):603-613. 


\section{REFERENCES}

232. Baldwin L, Abernethy P, Roberts L, Egan H. Forming, managing and sustaining alliances for health promotion. Health Promot J Austr 2005;16(2):138-43.

233. Butterfoss FD, Goodman RM, Wandersman A. Community coalitions for prevention and health promotion. Health Educ Res 1993;8(3):315-30.

234. Leurs MTW, Mur-Veeman IM, Van der Sar R, et al. Diagnosis of sustainable collaboration in health promotion: a case study. Health Promot Int 2006; submitted.

235. Leurs M, Mur-Veeman I, Schaalma H, De Vries N. Integrale samenwerking gaat verder dan de samenwerking tussen professionals: introductie van het DISC-model [Integrated collaboration continues beyond professional collaboration: introduction of the DISC-model]. Tijdschrift voor Gezondheidswetenschappen 2003;81:369-373.

236. Mur-Veeman I, Van Raak A. Inter-organizational networks on the Dutch home health care market. Int J Health Plann Manage 1994;9(3):245-58.

237. Peters LWH. De Netwerkbenadering. Review [The Network-approach - a review]. Woerden: NIGZ Center for Review and Implementation, 2001.

238. Ravensbergen J. ZON, ZonMw en effectieve implementatie. In: Ravensbergen J, Friele R, Keijsers J, Wensing M, Klazinga N, eds. In Zicht. Nieuwe wegen voor implementatie [In sight. New roads towards implementation]. Assen: Van Gorcum, 2003: 16-30.

239. Ruland E, Van Raak A, Spreeuwenberg C, Van Ree J. Managing New Public Health: hoe zijn preventieve samenwerkingsverbanden te realiseren? Een agenda voor actie en onderzoek. Tijdschrift voor Gezondheidswetenschappen 2003;81:52-55.

240. Van Raak A, Mur-Veeman I, Hardy B, Steenbergen M, Paulus A, eds. Integrated care in Europe. Description and comparison of integrated care in six EU countries. Maarssen: Elsevier gezondheidszorg, 2003.

241. Forsyth DR. Power. Group Dynamics 3 ed. Belmont, USA: Wadsworth Publishing Company, 1999: 207-234.

242. Greiner LE, Schein VE. Power and organization development: mobilizing power to implement change. Reading, Mass: Addison-Wesley, 1998.

243. Cummings TG, Worley CG. Organization development and change. Mason, OH: Thomson / South-Western College Publishing, 2001.

244. Van Wijnen JS, De Leeuw MA. GGD Zuidelijk Zuid-Limburg en het academisch ziekenhuis Maastricht. Naar een maximaal haalbare synergie. Utrecht: Ernst \& Young Consulting, 2000.

245. Forsyth DR. Leadership. Group Dynamics 3 ed. Belmont, USA: Wadsworth Publishing Company, 1999: 339-374.

246. De Jong J, Den Hartog D. Leadership as a determinant of innovative behaviour. A conceptual framework. SCALES Research report H200303. Zoetermeer: SCALES, 2003.

247. Hackett M, Spurgeon P. Leadership and vision in the NHS: how do we create the "vision thing"? Health Manpow Manage 1996;22(1):5-9.

248. Sullivan S, Glanz J. Building effective learning communities. Strategies for leadership learning \& collaboration. Thousand Oaks, CL: Corwin Press, 2006.

249. Van der Doelen FCJ, Klok PJ. Beleidsinstrumenten. In: Hoogerwerf A, Herweijer M, eds. Overheidsbeleid. Een inleiding in de beleidswetenschap. Alphen aan den Rijn: Samson 1998: 209-227.

250. Wilson JL. Leadership development: working together to enhance collaboration. $J$ Public Health Manag Pract 2002;8(1):21-6.

251. Tones K, Green J. Healthy public policy. In: Tones K, Green J, eds. Health promotion. Planning and strategies. London: Sage Publications, 2004: 175-207.

252. Steckler A, Goodman RM, Kegler MC. Mobilizing organizations for health enhancement In: Glanz K, Rimer BK, Lewis FM, eds. Health behavior and health education. Theory, research and practice. 3 ed. San Francisco: Jossey-Bass, 2002: 335-360. 
253. Bohlmeijer E, Ruland E, Van Raak A, Mur-Veeman I, eds. Procesmanagement in public health. Ontwerp, analyse en verandering. Utrecht: Trimbos-instituut, 2005.

254. Ford EW, Duncan WJ, Ginter PM. The structure of state health agencies: a strategic analysis. Med Care Res Rev 2003;60(1):31-57.

255. Hawe P, Noort M, King L, Jordens C. Multiplying health gains: the critical role of capacitybuilding within health promotion programs. Health Policy 1997;39(1):29-42.

256. McLean S, Feather J, Butler-Jones D. Building health promotion capacity. Action for learning, learning from action. Vancouver/Toronto: UBC-Press, 2005.

257. Allan J, Barwick TA, Cashman S, et al. Clinical prevention and population health. Am J Prev Med 2004:27:471-476.

258. Amodeo AR. Commentary: developing and retaining a public health workforce for the $21 \mathrm{st}$ century: readiness for a paradigm shift to community-based public health. J Public Health Manag Pract 2003;9(6):500-3.

259. Connelly J, Knight T, Cunningham C, Duggan M, McClenahan J. Rethinking public health: new training for new times. J Manag Med 1999;13(4-5):210-7.

260. Crisp BR, Swerissen H, Duckett SJ. Four approaches to capacity building in health: consequences for measurement and accountability. Health Promot Int 2000;15:99-107.

261. Latter S, Speller V, Westwood G, Latchem S. Education for public health capacity in the nursing workforce: findings from a review of education and practice issues. Nurse Educ Today 2003;23(3):211-8.

262. Lichtveld MY, Cioffi JP. Public health workforce development: progress, challenges, and opportunities. J Public Health Manag Pract 2003;9(6):443-50.

263. Mayer JP. Are the public health workforce competencies predictive of essential service performance? A test at a large metropolitan local health department. J Public Health Manag Pract 2003;9(3):208-13.

264. Potter MA, Barron G, Cioffi JP. A model for public health workforce development using the National Public Health Performance Standards Program. J Public Health Manag Pract 2003;9(3):199-207.

265. Riegelman RK, Evans CH, Garr DR. Why a clinical prevention and population health curriculum framework? Am J Prev Med 2004;27(5):477.

266. Riley BL. Dissemination of heart health promotion in the Ontario Public Health System: 1989-1999. Health Educ Res 2003;18(1):15-31.

267. Kelder SH, Mitchell PD, McKenzie TL, et al. Long-term implementation of the CATCH physical education program. Health Educ Behav 2003;30(4):463-75.

268. Plsek PE, Wilson T. Complexity, leadership, and management in healthcare organisations. Br Med J 2001;323(7315):746-9.

269. Conner M, Norman P. Predicting and changing health behaviour: future directions. Predicting health behaviour. 2 ed. Buckingham: Open University Press, 2005: 324-371.

270. Peters LWH, Leurs MTW, Jansen MWJ, Keijsers JFEM, Schaalma HP. Ontwikkeling van de schoolSlag checklist voor kwaliteit van schoolgerichte interventies. Tijdschrift voor Gezondheidswetenschappen 2004;82:50-57.

271. Van Wersch S, Winters-van der Meer S, Zomerplaag J, eds. Kwaliteitsmodellen in de zorgsector. Utrecht: Nederlandse Vereniging voor Kwaliteit en Zorg, 1999.

272. Argyris C. On organizational learning. 2 ed. Malden, USA: Blackwell Publishing 1999.

273. Jansen M, Feron F. Inventarisatie jongeren problematiek. Brief nr 94.AU.0082. Maastricht: GGD Zuidelijk Zuid-Limburg, 1994.

274. Jansen M. Die jeugd van tegenwoordig. Een onderzoek naar de omvang en de kenmerken van de Maastrichtse probleemjongeren. Maastricht: GGD Zuidelijk Zuid-Limburg, 1996. 


\section{REFERENCES}

275. Gemeente Maastricht B\&W, namens dezen H. Doedel. Medewerking aan onderzoek c.q. prevalentieschatting jongerenproblematiek in Maastricht. Brief nr B0-W/BOJ, 15 februari. Maastricht, Gemeente Maastricht/OCWS, 1995.

276. Gemeente Maastricht B\&W, namens dezen H. Doedel. Inventarisatie jongerenproblematiek. Brief 28 maart. Maastricht, Gemeente Maastricht/OCWS, 1995.

277. Jansen M. Een onderzoek naar de omvang en het risicogedrag van Maastrichtse probleemjongeren. Tijdschrift voor Alcohol en Drugspreventie 1995;21(3):163-164.

278. Jansen M. Risicogedrag en risicofactoren bij probleemjongeren. Verslag van de 15e Wilhelmina Rouwenhorst Lezing: De zichtbaarheid en inzichtelijkheid van preventieactiviteiten. Utrecht: Trimbos-instituut, 1997.

279. GGD Zuidelijk Zuid-Limburg. Maastrichtse probleemjongeren onder de loep genomen. Dagblad De Limburger 1995 May 31.

280. Van Duijnhoven YTHP. Epidemiology and control of sexually transmitted diseases in a Rotterdam STD clinic. PhD-thesis. Amsterdam: Universiteit van Amsterdam, 1997.

281. Van de Laar MJW. On the epidemiology of sexually transmitted diseases in the Netherlands. PhD thesis. Amsterdam: Universiteit van Amsterdam, 1997.

282. Henquet CJ, Jansen MW, Buwalda PJ, Neumann HA. Seksueel overdraagbare aandoeningen in Limburg in 1997; prevalentie volgens een enquete onder huisartsen en specialisten en volgens opgave van microbiologische laboratoria [Sexually transmitted diseases in Limburg in 1997; prevalence according to a survey of family practitioners and specialists and according to reports from microbiological laboratories]. Ned Tijdschr Geneeskd 2000;144(13):608-12.

283. Henquet CJM, Buwalda PJS, Jansen M. SOA in Limburg. Nederlands Tijdschrift voor Dermatologie \& Venereologie 1998;8(4):178.

284. Gemeente Maastricht. Gemeentelijk kansspelautomatenbeleid. Maastricht: Gemeente Maastricht, 1991.

285. Jansen M. Gemeentelijk kansspelautomatenbeleid ter preventie van gokverslaving en de rol van de GGD. Utrecht: Vereniging van Directeuren van Basisgezondheidsdiensten, 1993.

286. GGD Zuidelijk Zuid-Limburg. Reddingsoperatie MVV. Brief aan College van Burgemeester en Wethouders. Maastricht, 2003.

287. Van de Beek J. Economen UM: opbrengst casino Maastricht nihil. Maastricht mikt op verplaatsing casino. Dagblad De Limburger 2006 Febuary 16

288. Velthuis O. Voorkom harde concurrentie op de gokmarkt. Donner weigert Maastricht vergunning voor casino. De Volkskrant 2006 Febuary 11, p. 9.

289. Hoeijmakers M, De Leeuw E, Kenis P, De Vries NK. Local health policy development process - an expanded toolbox for health promotion. Health Promot Int 2007, in press

290. Jansen M. Gokken en behendigheid uit elkaar halen. Nederlandse Gemeenten 1992.

291. Jansen M. Mix van verminderen, voorkomen en hulpverlening is de beste medicijn voor verslaafden aan fruitautomaat. Ingezonden brief Binnenlands Bestuur 1992.

292. Jansen M. Opzetten van kansspelautomatenbeleid vereist veel politieke moed. Tijdschrift Gezondheidsvoorlichting 1993;10(6):9-10.

293. Jansen M. De GGD contra de moderne junk. Preventie van gokverslaving. GGD-Nieuws 1993(3):9-10.

294. Jansen MWJ, Lieber FWJ. Krassa, kassa! Advies van LVGGD en NeVIV over krasloterij. GGD-Nieuws 1994(7):17-19.

295. Boon DGB, Dols KJM. Invloed van verlengde openingstijden van amusementscenters in Maastricht op het speelgedrag. Maastricht: GGD Zuidelijk Zuid-Limburg, 2000.

296. Gezondheidsraad [The Health Council of the Netherlands]. Advies inzake hepatitis B. Den Haag: Staatsuitgeverij, 1983. 
297. Gezondheidsraad [The Health Council of the Netherlands]. Bescherming tegen hepatitis B. Rijswijk: Gezondheidsraad Commissie Hepatitis B, 1996.

298. Beuker RJ, Berns MPH, Van Rozendaal CM, et al. Surveillance van HIV-infecties onder injecterende druggebruikers in Nederland: metingen Heerlen/Maastricht 1998/99. Bilthoven: RIVM, 2001.

299. Carsauw HHC, Van Roozendaal CM, Scheepens JMFA, et al. Infecties met HIV, HBV en HCV onder injecterende druggebruikers in Heerlen/Maastricht. Bilthoven: RIVM, 1997.

300. Van Steenbergen JE, Van der Burgt M, Waldhober Q. Proefproject haalbaarheid vaccinatie van risicogroepen Hepatitis B. Utrecht: Landelijke Coördinatiestructuur Infectieziektebestrijding, 2001.

301. ZonMw. Verslag consensusbijeenkomst hepatitis B vaccinatie, 21 nov 2005. Den Haag: ZonMw, 2005.

302. GGD Nederland. Advies Landelijke vaccinatiecampagne hepatitis B gedragsgebonden risicogroepen, voorzetting na 2006. Utrecht: GGD Nederland, 2005.

303. GGD Nederland. Nieuwsbrief artikelen. HBV Campagne Nieuws, 2002-2006.

304. Jansen M, Jacobs E, Siedenburg E. Hepatitis B vaccinatie voor prostituees. SOA Bulletin 2002;23(2):10-12.

305. Van der Burgt M, Siedenburg E, Jansen M, Van Steenbergen J. Opmaat naar hepatitis-B preventie onder risicogroepen. SOA Bulletin 2000;21(4):4-6.

306. Van Steenbergen JE. Results of an enhanced-outreach programme of hepatitis B vaccination in the Netherlands (1998-2000) among men who have sex with men, hard drug users, sex workers and heterosexual persons with multiple partners. J Hepatol 2002;37(4):507-13.

307. De Wit GA, Kretzschmar M, Mangen MJJ. Is de vaccinatie van risicogroepen tegen hepatitis B kosten-effectief. Abstract Nederlands Volksgezondheidscongres 2006. Tijdschrift voor Gezondheidswetenschappen 2006;84:37.

308. Mangen J, Kretzschmar M, De Wit GA. Voor welke risicogroepen is vaccinatie tegen hepatitis B het meest kosten-effectief? Abstract Nederlands Volksgezondheidscongres 2006. Tijdschrift voor Gezondheidswetenschappen 2006;84:30.

309. Leurs M, Steenbakkers M, Jansen M, eds. Het SchoolSlag praktijkboek. Samenwerken aan preventie op maat. Maastricht: GGD Zuid Limburg, 2006.

310. Kassander R. Preventie in de Openbare Geestelijke Gezondheidszorg voor Maastricht en omgeving. Een regio-analyse. Maastricht: Universiteit Maastricht, 1999.

311. Leurs M, Jansen M. Schoolbeat, the new challenge. Network News. The European Network of Health Promoting Schools 2002(7):26-28.

312. Kleijnen R, Pennartz A, Leurs M. Een klasse apart. Integrale ketenzorg voortgezet onderwijs Maastricht e.o. Maastricht: Stichting Vorming, 2003.

313. Sniekers J, Buijs G, Leurs M. Op weg naar een gezonde school. Woerden: NIGZ/SLO, 2004.

314. Ruland E. Hartslag operationeel scenario. Maastricht: GGD Zuidelijk Zuid Limburg, 1998.

315. Bemelmans WJE, Wendel-Vos GCW, Bos G, Schuit AJ, Tijhuis MAR. Interventies ter preventie van overgewicht in de wijk, op school, op het werk en in de zorg. Een verkennende studie naar de effecten. Bilthoven: RIVM, 2004.

316. Schuit AJ, Wendel-Vos GC, Verschuren WM, et al. Effect of 5-year community intervention Hartslag Limburg on cardiovascular risk factors. Am J Prev Med 2006;30(3):237-42.

317. Wendel-Vos GCW, Ooijendijk WTM, Van Baal PHM, et al. Kosteneffectiviteit en gezondheidswinst van behalen van beleidsdoelen bewegen en overgewicht. Bilthoven: RIVM, 2005.

318. Harting J. Individual life style advice: development, implementation, and evaluation within the Hartslag Limburg cardiovascular prevention project. PhD-thesis. Maastricht: Maastricht University, 2005. 


\section{REFERENCES}

319. Harting J, Van Assema P, De Vries NK. Patients' opinions on health counseling in the Hartslag Limburg cardiovascular prevention project: perceived quality, satisfaction, and normative concerns. Patient Educ Couns 2006;61(1):142-51.

320. Harting J, Van Assema P, Ruland E, et al. Implementation of an innovative health service; a "real-world" diffusion study. Am J Prev Med 2005;29(2):113-9.

321. Harting J, Van Assema P, Van der Molen HT, Ambergen T, de Vries NK. Quality assessment of health counseling: performance of health advisors in cardiovascular prevention. Patient Educ Couns 2004;54(1):107-18.

322. Harting J, Van Assema P, Van Limpt P, et al. Cardiovascular prevention in the Hartslag Limburg project: effects of a high-risk approach on behavioral risk factors in a general practice population. Prev Med 2006;43(5):372-8.

323. Harting J, Van Assema P, Van Limpt P, et al. Effects of health counseling on behavioural risk factors in a high-risk cardiology outpatient population: a randomized clinical trial. EurJ Cardiovasc Prev Rehabil 2006;13(2):214-21.

324. Ronckers ET, Groot W, Ament AJ. Systematic review of economic evaluations of smoking cessation: standardizing the cost-effectiveness. Med Decis Making 2005;25(4):437-48.

325. Ronckers ET, Groot W, Steenbakkers M, Ruland E, Ament A. Costs of the 'Hartslag Limburg' community heart health intervention. BMC Public Health 2006;6:51.

326. Ronda G, Van Assema P, Candel M, et al. The Dutch Heart Health Community Intervention 'Hartslag Limburg': effects on smoking behaviour. EurJ Public Health 2004;14(2):1913.

327. Ronda G, Van Assema P, Candel M, et al. The Dutch Heart Health community intervention 'Hartslag Limburg': results of an effect study at individual level. Health Promot Int 2004;19(1):21-31.

328. Ronda G, Van Assema P, Ruland E, Steenbakkers M, Brug J. The Dutch heart health community intervention 'Hartslag Limburg': design and results of a process study. Health Educ Res 2004;19(5):596-607.

329. Ronda G, Van Assema P, Ruland E, Steenbakkers M, Van Ree J, Brug J. The Dutch heart health community intervention 'Hartslag Limburg': results of an effect study at organizational level. Public Health 2005;119(5):353-60.

330. Ruland E, Van Assema P, Ament A, Gorgels T, Van Ree J. Hartslag Limburg: integrale gezondheidsbevordering in buurten, gemeenten, bij huisartsen en in het ziekenhuis. Tijdschrift voor Gezondheidswetenschappen 2006;84:83-89.

331. Van Assema P, Ronda G, Steenbakkers M, Quaedvlieg M, Brug J. The reach of a computer-tailored nutrition education program in the Dutch heart health community intervention "Hartslag Limburg". J Nutr Educ Behav 2006;38(5):293-7.

332. Van Assema P, Steenbakkers M, Rademaker C, Brug J. The impact of a nutrition education intervention on main meal quality and fruit intake in people with financial problems. $J$ Hum Nutr Diet 2005;18(3):205-12.

333. Van Assema P, Steenbakkers M, Stapel H, Van Keulen H, Ronda G, Brug J. Evaluation of a Dutch public-private partnership to promote healthier diet. Am J Health Promot 2006;20(5):309-12.

334. Spreeuwenberg C, Van Ree J, Ruland E. Managing new public health: van preventieproject hart- en vaatziekten naar bestuurlijke verankering. Den Haag: ZonMw, 2002.

335. Nederlandse Hartstichting. Verslag van de conferentie Hartslag Limburg, over bruggen, pijlers en ankers in lokale gezondheidsbevordering, 10 november 2005. In: NHS, ed. Hartslag Limburg. Den Haag: NHS, 2005.

336. Horstman K, Houtepen R. Worstelen met gezond leven. Ethiek in de preventie van harten vaatziekten. Amsterdam: Het Spinhuis, 2005. 
337. Ansari WE, Weiss ES. Quality of research on community partnerships: developing the evidence base. Health Educ Res 2006;21:175-180.

338. McQueen DV. Perspectives on health promotion: theory, evidence, practice and the emergence of complexity Health Promot Int 2000;15:95-97.

339. Tang KC, Ehsani JP, McQueen DV. Evidence based health promotion: recollections, reflections, and reconsiderations. J Epidemiol Community Health 2003;57(11):841-3.

340. Glasgow RE, Lichtenstein E, Marcus AC. Why don't we see more translation of health promotion research to practice? Rethinking the efficacy-to-effectiveness transition. Am J Public Health 2003;93(8):1261-7.

341. Johnson K, Hays C, Center H, Daley C. Building capacity and sustainable prevention innovations: a sustainability planning model. Eval Program Plann 2004;27(2):135-149.

342. Pluye P, Potvin L, Denis J-L. Making public health programs last: conceptualizing sustainability. Eval Program Plann 2004;27(2):121-133.

343. Scheirer MA. Is sustainability possible? A review and commentary on empirical studies of program sustainability. American Journal of Evaluation 2005;26:320-347.

344. Shediac-Rizkallah MC, Bone LR. Planning for the sustainability of community-based health programs. Conceptual frameworks and future directions for research, practice and policy. Health Educ Res 1998;13:87-108.

345. Bilodeau A, Filion G, Labrie L, Bouteiller D, Perreault M. La perennisation des programmes de promotion de la santé: est elle possible? Le cas de quatre entreprises privé quebecoises de travailleurs cols blues [Is it possible to sustain health promotion programs in private companies? The case of four Quebec private companies of blue collar workers.]. Can J Public Health 2005;96(2):114-20.

346. Collins J. Good to great. Waarom sommige bedrijven een sprong vooruit maken en andere niet. Amsterdam/Antwerpen: Business Contact, 2006.

347. De Caluwé L, Vermaak H. Learning to change. A guide for organization change agents. Thousand Oaks: Sage Publications, 2003.

348. Swerissen H, Crisp BR. The sustainability of health promotion interventions for different levels of social organization. Health Promot Int 2004;19(1):123-130.

349. Wolff T. A practitioner's guide to successful coalitions. Am J Community Psychol 2001;29(2):173-91; discussion 205-11.

350. Lake JL, Peterson EA. An alternative structure for improving the public's health. J Public Health Manag Pract 2002;8(1):75-82.

351. Bakes-Martin R, Corso LC, Landrum LB, Fisher VS, Halverson PK. Developing national performance standards for local public health systems. J Public Health Manag Pract 2005;11(5):418-21.

352. Green LW. Public health asks of systems science: to advance our evidence-based practice, can you help us get more practice-based evidence? Am J Public Health 2006;96(3):406-9.

353. Bauer G, Davies JK, Pelikan J, Noack H, Broesskamp U, Hill C. Advancing a theoretical model for public health and health promotion indicator development: proposal from the EUHPID consortium. Eur J Public Health 2003;13(3 Suppl):107-13.

354. Smid H. VTV-2006 en zorgbalans in onderzoeksperspectief. Tijdschrift voor Gezondheidswetenschappen 2006;84(7):385-386.

355. Goodson P, Gottlieb NH, Smith MM. Put prevention into practice. Evaluation of program initiation in nine Texas clinical sites. Am J Prev Med 1999;17(1):73-8.

356. Goodwin MA, Zyzanski SJ, Zronek S, et al. A clinical trial of tailored office systems for preventive service delivery. The Study to Enhance Prevention by Understanding Practice (STEP-UP). Am J Prev Med 2001;21(1):20-8. 


\section{REFERENCES}

357. De Hollander AEM, Hoeymans N, Melse JM, Van Oers JAM, Polder JJ, eds. Zorg voor gezondheid. Volksgezondheid Toekomstverkenning 2006. RIVM rapport nr 270061003. Houten: Bohn Stafleu Van Loghum, 2006.

358. Ministerie van Volksgezondheid Welzijn en Sport. Preventienota Kiezen voor gezond leven. Den Haag: Ministerie Volksgezondheid, Welzijn en Sport, 2006.

359. Canadian Health Service Research Foundation. Weighing up the evidence. Making evidence-informed guidance accurate, achievable, and acceptable. Ottawa: Canadian Health Service Research Foundation, 2006.

360. Leischow SJ, Milstein B. Systems thinking and modeling for public health practice. Am J Public Health 2006;96(3):403-5.

361. Smith TW, Orleans CT, Jenkins CD. Prevention and health promotion: decades of progress, new challenges, and an emerging agenda. Health Psychol 2004;23(2):126-31.

362. Trochim WM, Cabrera DA, Milstein B, Gallagher RS, Leischow SJ. Practical challenges of systems thinking and modeling in public health. Am J Public Health 2006;96(3):538-46. 


\section{Summary}

Chapter 1 provides an introduction to this thesis. It highlights the considerations and motives that led to the decision to study the lack of coherence between public health policy, practice and research in the Netherlands. Public health has often been defined in terms of its aim and goals rather than by referring to any specific body of knowledge. Public health includes: (i) policy, as it is inherently a political enterprise; (ii) practice, as policies need to be implemented to create social action and to organize service delivery and (iii) research, to assess the prevalence and determinants of health problems and to investigate effectiveness and cost-benefit ratio of implemented activities. As the Netherlands are being confronted with a decline in healthy life expectancy compared to other European countries, the need for public health evidence is obvious. However, the evidence about public health actions which are aimed to improve people's health inevitably raises ethical, technical, practical, political, organizational and economic issues, making the evidence extremely complex. The assumption that collaboration between practice, research and policy will result in more solid evidence and higher quality standards in public health is widely supported. The complexity of evidence stresses the need for sustained collaboration between practice, policy and research. In the course of history, a linkage between research, practice and policy proved not to be an easy one. Currently, concerted actions by policy, practice and research are still rare.

Against this background, chapter 1 outlines the research questions, design and methods of our study. Some background information is given on the development of public health policy, practice and research during the last decades. This dissertation focuses on the question what barriers are preventing an interlinked development and how these barriers can be overcome. We restrict ourselves to the collaboration process at the local level. A multiple-case study is used to answer the research question. Six cases of intended collaborations between the policy, practice and research are included.

Chapter 2 contains an analysis of the work cycles of policy, practice and research, as they are carried out by the respective professionals. A work cycle shows a circular development pattern, with a succession of the following four steps: problem definition, approach formulation, implementation and interpretation. At first sight, the work cycles in these three domains seem to have much in common. However, the analysis shows that the lack of connections between the work cycles can be traced back to ambiguous and conflicting goals and 
expectations, having their origin in the niche character of the component areas of public health. Thirteen disparities appeared between the work cycles of policy, practice and research. These disparities range from priorities in problem statements to legitimate power roles, appraisal of evidence, work attitudes, work pace, transparency of final goals and the attention devoted to evaluation and continuation. Within the policy domain, public health plans can be made in the first year of an alderman's four-year term of office, after which policy effects must be available in the fourth year. In the academic discourse, public health problems are generally studied in a context separate from the hectic atmosphere and pressure of the political arena. The practice domain is confronted with day-to-day problems and the continuous search for standards of practical utility as a part of public health policy. The difference in paces makes collaboration extremely complicated.

We conclude that the individual behaviours of policy makers, practitioners and researchers are incompatible, unless an interface is created that can overcome the differences. Building such an interface requires strategies to stimulate compatibility at the administrative, institutional and individual levels, which could help to unite the relevant niche perspectives. The three different system levels are distinguished because of hierarchic procedures within local policy. These system levels correspond with strategic, tactic and operational policy making. The main actors at the different system levels are introduced.

Chapter 3 describes a logic model that distinguishes the relevant determinants of collaboration at each system level, i.e., the administrative, institutional and individual level. The Intervention Mapping framework is used to systematically apply theories and empirical evidence to decide on practical strategies which are expected to change the determinants and subsequently address the current lack of collaboration. The chapter describes the selection of practical strategies based on theories. The main determinants at the administrative level are power, leadership and resources. At the institutional level, the determinants include management support for organizational development, leadership, climate and culture of the organization and capacity building to promote collaboration. These conditions result from performance objectives for managers at the institutional level and may remove barriers between the work cycles, thus promoting collaboration. The main individual determinants relating to collaboration are knowledge, attitude, perceived social expectation, outcome expectation, behavioural capability and self-efficacy. For each determinant theoretical background information is given to motivate the selection of practical strategies. We present a set of thirty theory-based practical strategies that may enhance opportunities for collaboration. This set of proposed practical strategies should not be regarded as a general systematic roadmap from the present to the future for each collaboration process. An important aspect of Intervention Mapping is its 
tailoring, which implies that each problem, e.g., a collaboration process, needs to be studied as it is. Doing this may result in another set of practical strategies than the one described here. The selected practical strategies need to be organized into a programme that serves as a working tool during the collaboration process.

Chapter 4 evaluates the theory-based practical strategies with the help of the opinions of experts from the field of public health practice, policy and research. The goal of the expert meeting was to examine practical strategies for collaboration between policy, practice and research proposed by experts and to compare the degrees of priority they gave to these strategies with the theory-based practical strategies reported on in chapter 3 . The chapter describes the design of the meeting with the experts, its results and how we reached consensus about the practical strategies. Although the practical strategies mentioned by the experts and those based on theory were described differently, the meaning each was given highly corresponded. Discrepancies were solved by redefining the practical strategy by matching the meaning of experts and theory. The overview of redefined strategies was used as a checklist to evaluate the multiple-case study.

Chapter 5 describes the main results of our study. It examines the nature and extent of the application of the thirty practical strategies in six different public health cases. The six cases included in this dissertation are intended collaborations between the policy, practice and research domains (the niches) during the period 1991 to 2005. Together, these cases are representative examples of public health problems and offer clear illustrations of public health problems in general. We assessed the implementation of strategies over time in general terms, varying from intensive/good implementation $(+)$, mixed implementation $(+-)$, to no implementation $(-)$. An overall sum score for the use of practical strategies was calculated and interpreted against the context of the case concerned.

The results of the multiple-case study showed that three of the six cases were successful in terms of continuation beyond the initial period of projectspecific extra funding. The results also unequivocally showed that collaboration cannot be sustained beyond the initial period when institutional and administrative changes lag behind the individual changes. Administrative, institutional and individual changes keeping in step with each other seemed to be beneficial for successful collaboration. Successful collaboration between the niches can be achieved when various and multiple practical strategies are applied at each system level. The decisive number and specific type may differ in each case and may also depend on the context. Successful collaboration implied repeated interconnections between the work cycles of policy, research and practice. We 
conclude that coherence between the system levels of the three niches is necessary. Coherence means that there is vertical communication between the actors of the system levels, and horizontal communication between the actors of policy, practice and research at each system level and sometimes across the system levels. Coherence between the system levels can also be described in terms of strategic, tactic and operational management. Actors who discuss the results of the research work cycle may decide to enter these results into the practice and policy work cycle and vice versa. However, even such coherence did not guarantee success in the long run, as was shown in one out of the three successful cases.

In chronological terms, there was a shift from attention to individual strategies towards attention to institutional strategies, with the GGD obviously having learned from previous experience. Exactly these institutional strategies which focus on organizational development contributed to success. The benefits of these learning processes should be disseminated within the field of public health to prevent others from making the same mistakes.

Our study revealed that there was a lack of clarity about who should assume the role of process manager at the nstitutional level: the civil servant who is in charge of public health, the civil service manager, or the GGD? In addition, the corresponding tasks and responsibilities of the process manager were neither clearly defined nor allocated.

Performance indicators represent an important link between the goalsetting process and the reward system. Our study revealed that performance indicators combined with professional development to encourage professionals to collaborate with partners were undervalued by management. Strikingly, this was in direct contrast to the opinion of experts who judged that the strategy of using performance indicators was highly significant. There was a substantial missing in the use of performance indicators transcending the own organization, although improvements were realized from 1998 onwards.

The differences in time frames of the work cycles between practice, policy and research were not consciously handled beforehand. None of the cases showed anticipated planning procedures to better fit the paces of the policy, practice and research work cycles. The word coincidence is most appropriate to describe the correspondence in pacing if it occurred. Planning interconnections between the work cycles requires conscious prior planning by the professionals involved.

The assumption that successful collaboration between the three niches contributes to enhanced effectiveness, efficiency and efficacy of public health could not (or at least not yet) be unequivocally confirmed. The three successful cases showed more work cycle repetitions, associated with an incremental evolution towards improved effectiveness, efficacy and efficiency. The successful 
concerted actions between research, practice and policy seem to indicate an evolution towards improved public health evidence.

Chapter 6 contains a general discussion on the findings presented in the previous chapters and a consideration of the methodological quality issues. Despite some limitations the strength of the present study was the evaluation of the application of predefined practical strategies in six different public health cases. Using theoretical underpinning and experts' opinions we developed a set of practical strategies that was assumed to be effective in the promotion of collaboration between public health practice, policy and research. Based on a multiple-case study, we were able to draw conclusions about the potential of these practical strategies to overcome the barriers between the niches. The use of practical strategies to evaluate the collaboration process is innovative and has provided new insights, most importantly concerning the relevance of organizational development strategies.

Finally, this chapter draws conclusions and provides recommendations for practice, policy and research. Effective public health ideally requires that the policy, practice and research domains work as collaborative and mutually dependent partners, uniting many different disciplines. This study has shown that the barriers between the niches of practice, policy and research can be overcome when (1) a coherent application of practical strategies at the different system levels can be achieved; (2) organizational development strategies addressing collaboration are carried out within and across the niches which also clearly define and allocate the role of the process manager; (3) additional performance indicators are defined beyond one's own organization; (4) practical strategies are selected that stimulate reframing and are context-sensitive; and (5) more attention is given to the interconnections between the three work cycles well before implementation. Although this study did not involve sufficient follow-up time for the different collaborative projects to convincingly show that collaboration leads to more evidence-based public health, it did produce some indication of how more effective public health could be achieved. Efforts to increase the available knowledge on evidence-based public health would seem to benefit from a 'helical' approach, consisting of a complex set of interconnections between the research, practice and policy work cycles.

In the case of a programme that is intended to be sustained after a trial to achieve public health effects, we recommend that the decision to start should be linked to the decision to continue (or at least the intent to continue). Furthermore, at the institutional management level investing in time, priority setting and competencies is necessary to address collaboration strategies, particularly those that transcend the niches. A leading role for the GGD as process manager of collaboration programmes is proposed. A new kind of accountability in public health, which is related to the collaborative process, is recom- 


\section{SUMMARY}

mended. The creation of combined part-time positions within the research, policy and practice domains may yield mutual benefits. The Netherlands Organisation for Health Research and Development (ZonMw) is advised to offer the opportunity to incorporate the explicit goal of collaboration between the public health domains in their funding programmes. The set of practical strategies which was developed in our study should be considered as a practical, and constantly changing, instrument that fits the nature and the progress of the collaboration. The most important premise underlying all practical strategies is the willingness among the actors of the niches to reflect on their own frame of reference to overcome the barriers. For each work cycle, recognition of a problem is the starting point for further action. Therefore, immediately after local elections top-level consultations between the alderman, the professor of public health and the GGD-director are recommended to decide on concerted actions for the period until the next elections. Consensus about the first step of the work cycle, i.e. the problem, will result in running the work cycles simultaneously and allowing them to connect with each other when needed. Finally, we recommend achieving agreement on long-term follow-up procedures, in order to develop potentially effective public health programmes that can be sustained within public health policy and practice. Such long-term collaboration would correspond with the gradual developments in evidence-based public health.

The creation of 'Academic Collaborative Centres for Public Health' (In Dutch: Academische Werkplaats) throughout the Netherlands offers valuable opportunities to achieve collaborative efforts in which the strengthening of local public health policy forms a starting point for action. 


\section{Samenvatting}

Centraal in dit proefschrift staat de afstemming tussen beleid, praktijk en onderzoek op het terrein van de openbare gezondheidszorg. Openbare gezondheid wordt in het Engels aangeduid met de term 'public health' (= publieke gezondheid). De termen openbare gezondheidszorg, publieke gezondheid en publieke gezondheidszorg worden in dit proefschrift afwisselend gebruikt, steeds met dezelfde betekenis.

De openbare gezondheidszorg is vooral gericht op gezondheidsbevordering, gezondheidsbescherming, ziektepreventie en acute hulpverlening in geval van ongevallen en rampen. Uitgangspunt is telkens: 'Voorkomen is beter dan genezen'. Het gaat om het verbeteren van de volksgezondheid, en dus om het bevorderen van de kwaliteit van leven, het streven naar een lang én gezond leven, en het voorkomen van bedreiging of verslechtering van de gezondheid van risicogroepen. De openbare gezondheidszorg onderscheidt zich van de reguliere gezondheidszorg in die zin dat de reguliere zorg zich richt op behandeling ter genezing ('cure') en via verzorging ('care') van patiënten op het moment dat hun gezondheid al is geschaad. Het initiatief tot het aanbieden van openbare gezondheidszorg ligt bij de overheid. De overheid treedt actief en ongevraagd op. Op landelijk niveau bestaat de Wet Collectieve Preventie Volksgezondheid, waarin in algemene bewoordingen is aangegeven waaraan lokale overheden uit het oogpunt van volksgezondheid dienen te voldoen. De concrete uitvoering van deze wet wordt overgelaten aan de gemeenten. De gemeenten financieren deze collectieve en preventieve zorg zelf, in tegenstelling tot de reguliere, op het individu gerichte gezondheidszorg, die volledig uit het zorgverzekeringsstelsel wordt betaald. De Inspectie voor de Gezondheidszorg controleert of gemeenten de wet naar behoren uitvoeren. Op gemeentelijk niveau raakt de zorg voor de volksgezondheid aan allerlei andere beleidsterreinen, zoals wonen, werken, welzijn, onderwijs, milieu en verkeer. Beslissingen die op deze terreinen worden genomen, beïnvloeden tevens de publieke gezondheid. Door het voeren van facetbeleid probeert de overheid zich hiervan rekenschap te geven. De openbare gezondheidszorg begeeft zich hiermee ook buiten de keten van gezondheidszorginstellingen. Sterker nog: zonder facetbeleid en samenwerking met andere partners heeft de openbare gezondheidszorg maar beperkt resultaat. Cruciaal voor een adequate samenwerking is een voldoende onderlinge afstemming tussen beleid, praktijk en onderzoek. Dat de samenwerking op gemeentelijk niveau kan en moet worden verbeterd is het uitgangspunt van dit proefschrift. Deze samenwerking is geen doel op zich, maar een voor- 


\section{SAMENVATTING}

waarde voor een betere volksgezondheid in de toekomst. De in dit proefschrift beschreven studie richt zich op de vraag welke belemmeringen een betere afstemming en samenwerking tussen beleid, praktijk en onderzoek op het terrein van de openbare gezondheidszorg in de weg staan, en wat er voor nodig is om deze belemmeringen op te heffen.

De studie is gebaseerd op de analyse van een zestal voorbeelden uit de periode 1991 tot 2005. Elk voorbeeld betreft een onderzoek naar een 'public health' probleem met uitdrukkelijke praktijk- en beleidsrelevantie. Voor een deel zijn de opzet en uitkomsten van deze onderzoeken reeds in een eerder stadium in artikelvorm gepubliceerd. In deze oorspronkelijke publicaties stond het vakinhoudelijke perspectief centraal. Pas achteraf zijn deze zes onderzoeken geselecteerd om dienst te doen als 'casus' (voorbeeld-materiaal) voor de onderhavige studie. Als gevalsbeschrijvingen illustreren ze de zwakke en sterke punten in de afstemming en samenwerking tussen beleid, praktijk en onderzoek in de openbare gezondheidszorg: wat er mis gaat, en waarom dat gebeurt; maar ook hoe het goed kan gaan, en waarom. De zes voorbeelden behandelen de volgende thema's:

1. Probleemjongeren die regelmatig spijbelen, agressief zijn, regelmatig stelen uit winkels, overmatig alcohol en drugs gebruiken en thuis problemen hebben waardoor ze weglopen, vormen een verborgen groep. Om preventief jeugdbeleid te kunnen opzetten moet de omvang van de groep bekend zijn en dient er inzicht te zijn in de ernst en aard van hun problemen. Daarna kan de gemeente een programma opzetten en uitvoeren om deze problemen terug te dringen. Het onderzoek toonde aan dat 2 tot $3 \%$ van de Maastrichtse jongeren tot de risicogroep van probleemjongeren behoort, hetgeen overeenkomt met circa 300 tot 500 autochtone jongeren. De allochtone jongeren konden niet worden bereikt.

2. Sexueel overdraagbare aandoeningen (SOA) komen in toenemende mate voor, vooral onder jongeren beneden de 25 jaar. Hoeveel mensen jaarlijks last hebben van dergelijke aandoeningen is echter niet bekend. Evenmin is bekend welke artsen de meeste patiënten zien en waar de beste mogelijkheden liggen om voorlichting te geven ter preventie van herhaling van de klachten. Daarom werd onder alle huisartsen, gynaecologen, dermatologen en urologen in Limburg een schriftelijke enquête gehouden om exacte aantallen te achterhalen. Geconcludeerd werd dat de meeste patiënten (bijna $80 \%$ ) hun huisarts consulteren. Verder bleken de diagnostiek, de registratie en de behandeling van SOA's voor verbetering vatbaar. Zo werden partners vaak niet gewaarschuwd en werd vaak blind behandeld, dat wil zeggen dat medicatie werd gegeven zonder aanvullend onderzoek.

3. Door een onrustbarende toename van het aantal personen dat met de politie in aanraking komt als gevolg van gokproblematiek, nam de burgemeester van Maastricht in 1990 het initiatief om het gemeentelijke gokbe- 
leid te herzien. Onderzoek werd gedaan naar het aantal gokgelegenheden in de stad en de leeftijd van de bezoekers. Besloten werd voortaan geen gokautomaten meer toe te staan in zogenaamde laagdrempelige gelegenheden zoals sportkantines en snackbars, waar jeugd beneden de 18 jaar toegang heeft. Gokken is volgens de wet alleen toegestaan boven de 18 jaar. In 1999 vroegen de bestaande speelautomatenhallen een vergunning aan voor een verlenging van de openingstijden van 11 uur 's avonds tot 1 uur 's nachts. De gemeente Maastricht wilde graag inzicht hebben in de mogelijke negatieve effecten van deze maatregel, voordat tot honorering van de verzoeken om langer open te mogen blijven zou worden overgegaan. Onderzoek wees op een gering negatief effect. Een afweging van de economische, recreatieve en volksgezondheidsbelangen resulteerde uiteindelijk in het besluit de openingstijden te verlengen tot 1 uur 's nachts.

4. Hepatitis B is een ernstige en veel voorkomende aandoening onder homoen biseksuele mannen, prostituees, prostituanten en spuitende druggebruikers. Vanuit het Ministerie van Volksgezondheid, Welzijn en Sport (VWS) werd een landelijke proefcampagne gelanceerd om deze risicogroepen te vaccineren tegen hepatitis B. Onderzoek werd gedaan naar hoe deze moeilijk bereikbare groepen gemotiveerd kunnen worden tot een hepatitis B vaccinatie. Via diverse kanalen werd de doelgroep bereikt. De campagne bleek doeltreffend en doelmatig. Het landelijke beleid werd als reguliere gemeentelijke taak gecontinueerd.

5. Het bevorderen van de gezondheid van de jeugd is een belangrijke voorwaarde voor gezondheid op latere leeftijd. De school kan hierbij een belangrijke rol vervullen. Echter, in Nederland worden onderwijsinstellingen door tal van organisaties benaderd om uiteenlopende thema's onder de aandacht van de jeugd te brengen. Dit leidt tot versnippering en ineffectiviteit. Het programma Schoolslag is bedoeld om het aanbod van voorlichtingsprogramma's richting scholen te kanaliseren en kwalitatief te optimaliseren. De school wordt geïnformeerd over de feitelijke gezondheidstoestand van de leerlingen. Vervolgens krijgt de school ondersteuning bij het verhelderen van de prioriteiten en het maken van schoolgezondheidsplannen. Hiervoor is samenwerking tussen diverse partijen noodzakelijk. Onderzocht wordt hoe deze samenwerking kan worden vormgegeven en of de veranderingen ten goede komen aan de kwaliteit van het schoolgezondheidsbeleid en de gezondheid van de jeugd.

6. Hart- en vaatziekten vormen nog steeds een belangrijke oorzaak van ziekte en sterfte in Nederland, vooral in Zuid-Limburg. Dit gegeven vormde in 1998 de aanleiding om Hartslag Limburg te starten, een preventieprogramma voor mensen met risicogedrag (overgewicht, onvoldoende beweging, ongezonde voeding en roken) en voor patiënten die zich met hartklachten melden bij de huisarts of het ziekenhuis. In Maastrichtse achter- 


\section{SAMENVATTING}

standsbuurten werden veel laagdrempelige activiteiten uitgevoerd, variërend van dans-sessies tot voorlichting over de tekst op etiketten van voedingsmiddelen in de supermarkt. Patiënten kregen extra ondersteuning bij het aanleren van een gezonde leefstijl. Het programma bleek effectief. Binnen het gemeentelijke gezondheidsbeleid werd zes jaar lang prioriteit gegeven aan dit programma. Uit financiële en politieke overwegingen werd uiteindelijk afgezien van structurele uitvoering ervan. Alleen de leefstijladvisering in het ziekenhuis kon worden gecontinueerd.

Bij elk van de bovenstaande zes casus was sprake van naast elkaar opererende beleidsprocessen, uitvoeringspraktijken en onderzoeksactiviteiten. De afstemming daartussen was soms gebrekkig en de samenwerking verliep niet altijd succesvol. Op basis van een vooraf ontwikkeld theoretische raamwerk werden deze gevalsbeschrijvingen in een multiple-case study aan een gemeenschappelijke analyse onderworpen. De opeenvolgende hoofstukken van het proefschrift beschrijven dit analyseproces.

Hoofdstuk 1 introduceert het onderwerp van dit proefschrift: het gebrek aan afstemming tussen beleid, praktijk en onderzoek op het terrein van de publieke gezondheid. De overwegingen en motieven die aan dit onderzoek ten grondslag hebben gelegen, worden belicht. Meestal wordt de publieke gezondheid beschreven in termen van intenties of streefdoelen. Publieke gezondheid refereert niet naar een eigenstandig kennisgebied maar ontleent zijn identiteit aan een combinatie van diverse disciplines. Publieke gezondheidszorg omvat: 1. beleid, omdat het domein intrinsiek verbonden is met politieke keuzen, prioriteiten en beslissingen ten aanzien van de inrichting van de zorg; 2. praktijk, omdat het beleid uitgevoerd moet worden, bijvoorbeeld via het organiseren van voorlichting, sociale actie, dienstverlening en voorzieningen; en 3. onderzoek, om de aard en omvang van publieke gezondheidsproblemen in kaart te brengen, de determinanten ervan te identificeren, en het (potentiële) effect van het beleid en het handelen in de praktijk vast te stellen. Waar Nederland wat betreft de (gezonde) levensverwachting jarenlang tot de top vijf van de Europese landen behoorde, worden we de laatste jaren geconfronteerd met een onrustbarende achteruitgang op de ranglijst. We nemen tegenwoordig een middenpositie in in de rij van de 27 EU-landen. Om hierin verandering te kunnen aanbrengen is effectief beleid nodig op het terrein van de publieke gezondheidszorg. Echter, er is onvoldoende inzicht in wat er nodig is om van een effectieve publieke gezondheidszorg te kunnen spreken. Bovendien is onvoldoende duidelijk welke parameters bij het beoordelen van de effectiviteit in beschouwing genomen dienen te worden, en wat hierbij de rol is van bijvoorbeeld ethische, uitvoeringstechnische, praktische, organisatorische en economische aspecten. Zo is de discussie over de effectiviteit van het huidige rookbeleid gevoerd vanuit allerlei verschillende invalshoeken en met allerlei verschillende argumenten, variërend 
van individuele vrijheidsberoving tot economische gevolgen en praktische uitvoerbaarheid van de naleving van de nieuwe wetgeving. De bewijsvoering inzake de effectiviteit is dus uitermate complex. Juist omdat de effectiviteit van de publieke gezondheidszorg zo moeilijk valt aan te tonen, zeker op korte termijn, lijkt een langdurige en meer structurele samenwerking tussen beleid, praktijk en onderzoek geïndiceerd. Deze breed gedragen visie stoelt op de aanname dat een dergelijke samenwerking binnen de publieke gezondheidszorg vanzelf zal leiden tot een hogere kwaliteit en effectiviteit, en tevens tot beter inzicht hierin. Echter, de geschiedenis herbergt tal van voorbeelden waaruit blijkt dat deze samenwerking maar moeizaam tot stand komt. Ook momenteel is een dergelijke samenwerking tussen de drie domeinen relatief zeldzaam.

Tegen deze achtergrond worden in dit hoofdstuk de onderzoeksvraag, het onderzoeksontwerp en de methoden van onderzoek beschreven. Een kort historische schets van de ontwikkelingen binnen de publieke gezondheidszorg wordt gepresenteerd, van het overheidsbeleid in deze, de praktijkuitvoering en het onderzoek. De centrale vraagstelling van dit proefschrift gaat over de barrières tussen beleid, praktijk en onderzoek die een gezamenlijke ontwikkeling belemmeren en over hoe deze barrières geslecht kunnen worden. De vraagstelling van het onderzoek beperkt zich tot het lokale niveau, oftewel het gemeentelijke volksgezondheidsbeleid.

Hoofdstuk 2 analyseert de werkcycli van respectievelijk beleid, praktijk en onderzoek zoals deze door de binnen deze domeinen opererende professionals worden uitgevoerd. Een werkcyclus beschrijft de achtereenvolgende stappen die men doorgaans maakt om een probleem aan te pakken. Het gaat hierbij om vier stappen. Stap 1 begint met de (h)erkenning van een probleem. Vervolgens wordt in stap 2 het probleem ontrafeld, waarbij er vragen gesteld worden zoals: hoe vaak komt het probleem voor, bij wie, waarom, waardoor, wat kunnen we eraan doen? Er wordt een plan van aanpak opgesteld waarin wordt vastgelegd wie wat gaat doen, waar, wanneer, en hoe. In stap 3 wordt dit plan daadwerkelijk uitgevoerd. Stap 4 beschrijft de resultaten en mondt uit in een nieuwe beoordeling van probleem: is het probleem verminderd of niet? Samengevat bestaat de werkcyclus uit: probleemdefinitie, probleemanalyse en plan van aanpak, uitvoering, en interpretatie. Op het eerste gezicht lijken de werkcycli van praktijk, beleid en onderzoek sterk op elkaar. Ze zijn opgebouwd uit dezelfde stappen. Maar bij nadere analyse blijken er per stap grote verschillen en tegengestelde belangen te bestaan. Deze tegengestelde belangen zijn te herleiden tot het zogenaamde niche-karakter van beleid, praktijk en onderzoek. De term 'niche' komt uit de biologie en heeft betrekking op omgevingscondities die noodzakelijk zijn voor het instandhouden van de eigen soort. Naar analogie van het biologische niche-concept kunnen praktijk, beleid en onderzoek worden opgevat als aparte niches. Elke niche moet zichzelf in stand houden en creëert hiervoor 


\section{SAMENVATTING}

bepaalde omstandigheden waardoor samenwerking die zich uitstrekt over de eigen grenzen wordt bemoeilijkt. Zo heeft elke niche een eigen referentiekader, een eigen taal, eigen gedragscodes, een eigen tempo etcetera. De analyse van de verschillen tussen de werkcycli van beleid, praktijk en onderzoek bracht dertien ongelijkwaardigheden aan het licht. Deze varieerden van de wijze van prioriteitstelling van problemen tot het hebben van de formele macht, de waardering van bewezen effectiviteit, de werkattitude van de professionals, tempoverschillen in de werkcycli, transparantie van de te bereiken einddoelen, en aandacht voor evaluatie en continuiteit. De werkcyclus van het beleid duurt vier jaar. De cyclus vangt aan met de benoeming van een verantwoordelijke wethouder na de lokale verkiezingen en eindigt na vier jaar, als in de aanloop naar de nieuwe verkiezingen de beleidseffecten zichtbaar moeten zijn. De werkcyclus van een academisch (promotie) onderzoekstraject kan in gemiddeld vier jaar worden afgerond, maar is niet onderhevig aan de druk van politieke besluitvormingsprocessen. De werkcyclus van de praktijk is doorgaans veel korter, liefst zo kort mogelijk, aangezien praktijkprofessionals geconfronteerd worden met acute, dagelijkse problemen die bij voorkeur zo snel mogelijk opgelost moeten worden. Deze verschillen in duur van de werkcycli die kenmerkend zijn voor beleid, praktijk en onderzoek zorgen ervoor dat samenwerking uitermate ingewikkeld is.

Geconcludeerd wordt dat de individuele gedragingen van beleidsmakers, onderzoekers en praktijkprofessionals van nature moeilijk met elkaar verenigbaar zijn, tenzij er een samenwerkingsverband wordt aangegaan om de barrières te bespreken en te zoeken naar oplossingen. Binnen zo'n samenwerkingsverband is een plan van aanpak nodig om de partijen tot elkaar te brengen. Dit dient zowel op bestuurlijk (oftewel strategisch) niveau te geschieden, als op institutioneel (oftewel tactisch) en individueel (oftewel operationeel) niveau. Gegeven het hiërarchische karakter van de besluitvormingsprocedures over het gemeentelijke beleid is het noodzakelijk onderscheid te maken tussen deze drie systeemniveaus, aangezien op elk van de niveaus veranderingen nodig zijn.

In Hoofdstuk 3 wordt een zogenaamd 'logisch model' beschreven waarin de relevante factoren die bepalend zijn voor (het gebrek aan) samenwerking worden onderscheiden op de drie systeemniveaus, te weten het bestuurlijke, institutionele en individuele niveau. De methode van Intervention Mapping is gebruikt om systematisch theorieën en empirische gegevens te gebruiken voor de selectie van praktische strategieën. Praktische strategieën geven concreet inhoud aan het plan van aanpak uit hoofdstuk 2 en geven aan hoe men tot samenwerking kan komen. In dit hoofdstuk worden tevens de belangrijkste actoren op de drie onderscheiden niveaus beschreven. Op bestuurlijk niveau zijn gemeentebestuur, gemeenteraad, directies van instellingen en hoogleraren uit de onderzoekswereld, directeuren van onderzoeksinstituten, en/of programmaleiders 
binnen de onderzoeksorganisatiestructuur de aangewezen actoren. Op institutioneel niveau spelen directeuren van gemeentelijke diensten en van uitvoerende instellingen een belangrijke rol naast de vakgroepvoorzitters van de universiteit. Individuele praktijkprofessionals, onderzoekers en beleidsambtenaren zorgen voor de feitelijke werkuitvoering.

De belangrijke factoren op bestuurlijk niveau zijn macht, leiderschap en middelen. Op institutioneel niveau hebben de relevante factoren betrekking op ondersteuning van het management voor organisatie ontwikkeling, leiderschap, het klimaat van de organisatie, de cultuur van de organisatie en het opbouwen van capaciteit om tot samenwerking te komen. Of op dit niveau voldaan wordt aan de voorwaarden voor samenwerking, wordt in de eerste plaats bepaald door (het gedrag van) de managers. Op individueel niveau zijn de belangrijkste voorwaardenscheppende factoren voor samenwerking kennis, houding, ervaren sociale norm, uitkomstverwachting, bekwaamheid en vertrouwen in eigen kunnen.

Voor elk van de factoren op bestuurlijk, institutioneel en individueel niveau wordt beschreven welke theoretische kennis er beschikbaar is, en hoe op basis van deze kennis de selectie van een serie praktische strategieën gericht op bevordering van de samenwerking tot stand is gekomen. We presenteren een pakket van dertig, op theorie gebaseerde, praktische strategieën waarvan we verwachten dat deze de kansen op samenwerking bevorderen. Het gaat hierbij niet om een vast pakket dat past bij elke vorm van samenwerking, maar om een variabel pakket, waarvan de invulling afhankelijk is van de specifieke context waarin de samenwerking plaatsvindt. Een belangrijk aspect van Intervention Mapping is juist het op-maat maken van het plan van aanpak. Dit impliceert dat elke situatie waarbij de samenwerkingsverhoudingen in het geding zijn, afzonderlijk geanalyseerd moet worden, om te kunnen beoordelen welke combinatie van praktische strategieën in de gegeven sitatie het meest geëigend is.

In Hoofdstuk 4 wordt een vergelijking gemaakt tussen de theoretisch gefundeerde praktische strategieën zoals voorgesteld in hoofdstuk 3 en de opvattingen van experts uit het praktijkveld, de beleidssector en de onderzoekswereld over essentiële samenwerkingsstrategieën. Het doel van de expertbijeenkomst was te toetsen of de door experts voorgestelde praktische strategieën voor samenwerking tussen beleid, praktijk en onderzoek overeenkomen met de theoretisch gefundeerde praktische strategieën, zowel qua inhoud als prioriteitstelling. De opzet van de expertbijeenkomst wordt beschreven, en vervolgens de resultaten en de manier waarop consensus is bereikt over de praktische strategieën. Hoewel de door de experts voorgestelde en de uit de theorie afgeleide praktische strategieën verschillend waren geformuleerd, bracht een inhoudsanalyse een grote mate van overeenstemming aan het licht ten aanzien van de achterliggende betekenis van de strategieën. Indien nodig werd daarom gekozen voor 


\section{SAMENVATTING}

een herdefinitie van een bepaalde strategie, zodanig dat deze voldoende recht deed aan zowel de theorie als de mening van de experts. Het uiteindelijke resultaat was een pakket van dertig geherformuleerde praktische strategieën. Deze lijst met strategieën is vervolgens gebruikt als checklist om de kwaliteit van de samenwerking tussen beleid, praktijk en onderzoek te beoordelen in de context van de multiple-case study (hoofdstuk 5).

Hoofdstuk 5 beschrijft de belangrijkste uitkomsten van de multiple-case study. Gerapporteerd wordt in welke mate dertig eerder geïdentificeerde praktische strategieën gehanteerd zijn in zes verschillende casus. De zes casus zijn ontleend aan beoogde samenwerkingsverbanden tussen de niches van beleid, praktijk en onderzoek gedurende de periode 1991 tot 2005, binnen de gemeenten Maastricht, Margraten, Meerssen, Eysden en Valkenburg. De zes gekozen voorbeelden zijn exemplarisch voor de samenwerkingsproblemen die zich in de Nederlandse openbare gezondheidszorg kunnen voordoen. De toepassing van de praktische strategieën is in algemene termen geëvalueerd, aan de hand van de volgende beoordelingscategorieën: intensieve/goede implementatie (+; 2 punten), gemengde implementatie ( $+-; 1$ punt), geen implementatie ( $-; 0$ punten). Op basis van de scores per strategie is voor elke casus een totaal-score berekend. Interpretatie van deze overall-score vond plaats tegen de achtergrond van de betreffende casus.

De resultaten van de multiple-case study laten zien dat er in drie van de zes casussen succesvol is samengewerkt. Succesvolle samenwerking betekende dat de samenwerking ook na de proefperiode en na afloop van de tijdelijke projectfinanciering gecontinueerd werd. De resultaten laten verder duidelijk zien dat samenwerking niet beklijft na de proefperiode als veranderingen op individueel niveau niet gepaard gaan met institutionele en bestuurlijke veranderingen. Als bestuurlijke, institutionele en individuele veranderingen met elkaar in de pas blijven lijkt dit de samenwerking te bevorderen. Succesvolle samenwerking tussen de verschillende niches lijkt tot stand te kunnen komen indien meerdere en bovendien verschillende praktische strategieën worden toegepast op elk van de drie systeemniveaus. Het beslissende aantal en de specifieke soort kan per casus verschillen en is mede afhankelijk van de context. Succesvolle samenwerking impliceert tevens dat er herhaaldelijk verbindingen tussen de onderscheiden werkcycli tot stand komen. Geconcludeerd wordt dat samenhang tussen de systeemniveaus noodzakelijk is. Samenhang betekent hier dat er zowel verticale communicatieverbindingen zijn tussen de actoren die opereren binnen de drie systeemniveaus, als horizontale verbindingen tussen beleid, praktijk en onderzoek. Samenhang tussen het bestuurlijke, institutionele en individuele niveau wordt ook wel omschreven als strategische, tactische en operationele aansturing. Een dergelijke samenhang tussen de niches leidt ertoe dat de desbetreffende actoren met elkaar in dialoog raken. Als gemeentebestuur, hoog- 
leraren, directeuren van onderzoeksinstituten en directies van instellingen gezamenlijk de resultaten van bijvoorbeeld de onderzoekswerkcyclus bespreken kunnen zij meer gefundeerd besluiten of zij de onderzoeksuitkomsten in praktsche of beleidsmaatregelen willen vertalen. Om het onderzoek naar de probleemjongeren als voorbeeld te nemen: op basis van de uitkomst dat 2-3\% van de Maastrichtse jeugd tot de risicogroep van probleemjongeren lijkt te horen, kan in onderlinge samenspraak besloten worden of specifieke aandacht voor deze categorie in het kader van het gemeentelijke jeugdbeleid al dan niet wenselijk is. Andersom kunnen praktijkervaringen ook worden ingebracht in beslissingen over beleid en onderzoek. Zo laat het Schoolslag-voorbeeld zien dat op basis van signalen uit de praktijk besloten kan worden het schoolgezondheidsbeleid te profileren als apart onderdeel van het gemeentelijke gezondheidsbeleid en tevens dat het initiëren van onderzoek om de effectiviteit van dit beleid te evalueren hoge prioriteit krijgt.

Alle positieve bevindingen en potenties ten spijt, heeft dit onderzoek ook uitgewezen dat zelfs in geval van het realiseren van een geslaagde afstemming tussen strategische, tactische en operationele aansturing in beleid, praktijk en onderzoek, succes op de langere termijn nog niet gegarandeerd is. Eén van de drie casus die als succesvol werden bestempeld, het Hartslagproject, maakte dit duidelijk.

Gedurende de periode 1991-2005 is door de Zuid-Limburgse GGD het accent verschoven van individuele naar institutionele en bestuurlijke strategieën. Vooral de institutionele samenwerkingsstrategieën met aandacht voor organisatieontwikkeling blijken bij te dragen aan succes. Een lerende organisatie waar managers voorzien in de randvoorwaarden om te kunnen samenwerken biedt de beste kansen. Kennelijk heeft de GGD leergeld betaald voor eerdere ervaringen. Landelijke verspreiding van deze inzichten is noodzakelijk om te voorkomen dat GGD-en in hun ontwikkeling geremd worden.

Dit onderzoek laat verder zien dat er grote onduidelijkheid bestaat over welke partij op institutioneel niveau de rol van procesmanager dient in te vullen: de betreffende beleidsambtenaar, de directeur van de desbetreffende gemeentelijke dienst, of de GGD? Daarnaast zijn de bijbehorende taken en verantwoordelijkheden van de procesmanager niet helder geformuleerd.

Prestatie-indicatoren dienen om de doelstellingen van de organisatie en het waarderings- en beloningssysteem voor het personeel met elkaar in overeenstemming te brengen. Ons onderzoek laat zien dat er vanuit het management weinig aandacht is voor prestatie-indicatoren in combinatie met persoonlijke ontwikkelingsplannen om zo het personeel aan te moedigen tot samenwerking. Dit gebrek aan aandacht staat in schril contrast met de mening van experts, die aangaven zeer veel belang te hechten aan het hanteren van heldere prestatieindicatoren gericht op samenwerking. Prestatie-indicatoren die het niveau van 


\section{SAMENVATTING}

de eigen organisatie overstijgen waren hoegenaamd afwezig, hoewel er na verloop van tijd wel verbetering optrad.

Het probleem van de verschillende planningscycli en werktempo's compliceert de samenwerking. Beleid, praktijk en onderzoek werken elk met een eigen tijdshorizon. In geen van de casus is bij de planning van de werkprocessen in de verschillende domeinen voldoende geanticipeerd op deze tempoverschillen. Voor zover praktijkbevindingen al werden ingebracht bij de besluitvorming over beleid en onderzoek, ging het meestal om een toevalstreffer. Om verbindingen tussen de werkcycli van beleid, praktijk en onderzoek te kunnen realiseren is het noodzakelijk dat de professionals de werkprocessen vooraf op elkaar afstemmen. In het voorbeeld van schoolgezondheidsbeleid is dit nog het meest expliciet geprobeerd. De vierjaars termijn van gemeentelijk beleid is als uitgangspunt gebruikt voor de planning van de activiteiten in de verschillende domeinen. Dit betekent dat de presentatie van de schoolgezondheidsgegevens, het formuleren van prioriteiten, het maken en uitvoeren van schoolgezondheidsplannen en het evalueren ervan moet plaatsvinden gedurende een periode van vier jaar, die overeenkomt met de gemeentelijke beleidscyclus.

De aanname dat succesvolle samenwerking tussen de drie niches bijdraagt aan het vergroten van de kennis over de effectiviteit, doelmatigheid en doeltreffendheid van de publieke gezondheidszorg is niet - of ten minste nog niet overtuigend aangetoond in dit onderzoek. Bij de drie succesvolle casus was er wel sprake van terugkerende verbindingen tussen de werkcycli, waarbij resultaten van de ene werkcyclus werden ingevoerd in een andere werkcyclus, zodat er langzaam aan verbeteringen tot stand kunnen komen wat betreft de effectiviteit, doelmatigheid en doeltreffendheid. Zo zijn in het voorbeeld van hepatitis B de praktijkgegevens over het bereiken van de doelgroep betrokken bij het herformuleren van het beleid. Vervolgens is het aangepaste beleid uitgevoerd en op effectiviteit onderzocht. De onderzoeksgegevens hiervan zijn op hun beurt gebruikt voor een aanpassing van het landelijke en lokale beleid wat betreft de vaccinatie van risicogroepen. De succesvol georkestreerde interacties tussen beleid, praktijk en onderzoek lijken inderdaad gepaard te gaan met een verbetering van het effect van de publieke gezondheidszorg.

Hoofdstuk 6 bevat een algemene discussie over de onderzoeksresultaten en een kritische beschouwing over de methodologische kwaliteit van het onderzoek. Ondanks enkele tekortkomingen in de onderzoeksopzet schuilt de kracht van deze studie in het feit dat de bruikbaarheid van een aantal praktische samenwerkingsstrategieën is getest bij zes verschillende voorbeelden uit de publieke gezondheid. Een pakket van strategieën is samengesteld op geleide van theoretische kaders en meningen van experts. De verwachting was dat bij toepassing van deze strategieën de samenwerking tussen beleid, praktijk en onderzoek zou worden bevorderd. Op basis van een multiple-case study konden conclusies 
worden getrokken over het potentiële vermogen van deze strategieën om de barrières tussen de drie niches te slechten. Het gebruik van praktische strategieen om een samenwerkingsproces te evalueren is vernieuwend. Deze werkwijze heeft nieuw inzicht opgeleverd, vooral met betrekking tot het belang van organisatieontwikkelingsstrategieën.

Tot slot bevat dit hoofdstuk een aantal conclusies en worden aanbevelingen voor de toekomst gedaan. Om te komen tot effectieve publieke gezondheidszorg is het zaak te zorgen voor voldoende wisselwerking tussen beleid, praktijk en onderzoek, waarbij tal van disciplines elkaar kunnen ontmoeten. Dit onderzoek heeft aangetoond dat de kloof tussen beleid, praktijk en onderzoek overbrugbaar is als er 1 . een samenhangende toepassing van praktische strategieën op de drie systeemniveaus gerealiseerd kan worden; 2. organisatieontwikkelingsstrategieën worden toegepast die gericht zijn op samenwerking en het toewijzen en formuleren van de rol van procesmanager op institutioneel niveau; 3. prestatie-indicatoren worden geformuleerd die de eigen organisatie overstijgen; 4. praktische strategieën op maat worden vastgesteld, gericht op het kritisch beschouwen van het referentiekader van de niches; en 5. voorafgaand aan de start van samenwerking aandacht wordt gegeven aan de gewenste verbindingen tussen beleid, praktijk en onderzoek.

Op basis van de onderzoeksresultaten worden de volgende aanbevelingen gedaan. Indien het voor het bereiken van gezondheidseffecten nodig is dat een nieuw programma na de proefperiode gecontinueerd wordt, bevelen wij aan om hiermee reeds rekening te houden tijdens de initiële besluitvorming op bestuurlijk niveau. Het besluit om te starten dient, bij gebleken succes, tevens een besluit tot continuering in te houden of ten minste de intentie ertoe. Daarnaast bevelen wij aan om op institutioneel niveau prioriteit te geven aan en competenties te ontwikkelen gericht op samenwerkingstrategieën die de eigen organisatie overstijgen. Voorgesteld wordt om bij intersectorale samenwerking de directeur van de GGD, of een daartoe gemandateerde GGD-professional, als procesmanager op te laten treden. Nieuwe prestatie-indicatoren gericht op samenwerking zijn nodig. Als voorbeeld wordt de constructie genoemd waarbij onderzoekers, beleidsmakers en praktijkprofessionals op 50-50 basis worden aangesteld bij twee verschillende instellingen. Dit kan wederzijds voordeel opleveren voor de betrokken instellingen. Aan Zorgonderzoek Nederland ( $\mathrm{ZonMw}$ ) wordt het advies gegeven om samenwerking binnen het domein van de publieke gezondheid als expliciet doel in te bouwen in bestaande en nieuw te starten onderzoeksprogramma's. Praktische samenwerkingsstrategieën dienen afgestemd te zijn op de context waarin deze worden toegepast. De belangrijkste premisse is dat de toepassing van een praktische strategie bijdraagt aan een grotere bereidheid tot het kritisch reflecteren op de reguliere denk- en werkwijze om de kloof tussen praktijk, beleid en onderzoek te kunnen overbruggen. Bij elke werkcyclus is de (h)erkenning van een probleem het startpunt van hande- 


\section{SAMENVATTING}

len. Daarom wordt aanbevolen om direct na elke nieuwe ronde van lokale verkiezingen op bestuurlijk niveau gezamenlijk - dat wil zeggen met verantwoordelijke wethouder, de hoogleraar publieke gezondheid en/of de directeur van het 'public health' onderzoeksinstituut, en de GGD-directeur - onderhandelingen te voeren over het lokale gezondheidsbeleid en besluiten te nemen over gezamenlijke plannen voor de komende vier jaar. Consensus over de maatschappelijke, wetenschappelijke en praktische relevantie van een probleem vormt de eerste stap in een gezamenlijke aanpak. De werkcycli kunnen gelijktijdig starten of elkaar opvolgen waarbij resultaten van de ene werkcyclus benut kunnen worden in de andere werkcyclus. Ten slotte bevelen we aan om overeenstemming te bereiken over de vervolgstappen voor de lange termijn, zodat effectieve programma's kunnen worden ontwikkeld en geprolongeerd. Dit onderzoek heeft vooralsnog geen overtuigend bewijs geleverd voor de veronderstelling dat samenwerking automatische leidt tot 'evidence-based public health', hoewel er wel aanwijzingen in die richting zijn. Pogingen om de beschikbare kennis met betrekking tot evidence-based public health te vergroten lijken voordeel te hebben van een helix-achtige benadering. De helix bestaat uit regelmatig terugkerende verbindingen tussen de werkcycli van beleid, praktijk en onderzoek, vergelijkbaar met de waterstofbruggen tussen de baseparen in de DNA-helix. Een lange termijn programmering in de vorm van een helix spoort met de geleidelijke vorderingen die binnen de publieke gezondheidszorg gebruikelijk zijn.

De vorming van Academische Werkplaatsen voor de Publieke Gezondheid in Nederland biedt een uitstekende gelegenheid om samenwerking tussen beleid, praktijk en onderzoek te realiseren, waarbij versterking van het lokale gezondheidsbeleid het uitgangspunt moet zijn zijn.

Voor een uitvoerigere Nederlandstalige samenvatting, zie:

www.academischewerkplaatslimburg.nl 


\section{Dankwoord}

promoveren is fietsen op de Mont Ventoux, waar Tommy Simpson nog is overleden. onder zo tragiese omstandigheden werd hier de wereldkampioen doodmoe.

op deze col zijn velen losgereden, eerste categorie, sindsdien tabu. het ruikt naar dennegeur, Sunsilk Shampoo, die je wel nodig hebt, eenmaal beneden.

alles is onuitsprekelijk vermoeiend, de Mont Ventoux opfietsen wel heel erg, waarvoor ook geldt: bezint eer gij begint.

toch haal ik, ook al is de hitte schroeiend, de top van deze kaalgeslagen berg: ijdelheid en het najagen van wind.

Vrij naar Jan Kal (Haarlem 1946), Fietsen op de Mont Ventoux, 1974 (sonnettenbundel)

Rust roest. Daarom nam ik me een aantal jaren geleden voor ooit fietsend de Mont Ventoux te beklimmen, liefst vóór mijn 50. Daarom ook besloot ik op

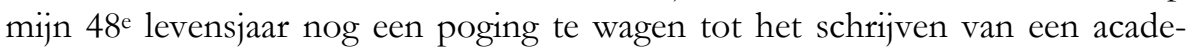
misch proefschrift. Met beide uitdagingen lijkt het uiteindelijk goed te komen. Tot op zekere hoogte bleken dit proefschrift en de Mont Ventoux vergelijkbare klimkoersen.

Menigeen zal zich afvragen waar al dat geklauter op die leeftijd nog goed voor is. Wat de dissertatie betreft had ik een duidelijk oogmerk. Ik wilde in de toekomst vooral blijven werken op het snijvlak van beleid, praktijk en onderzoek in de openbare gezondheidszorg. De vestiging van een Academische Werkplaats voor de GGD in de regio Zuid-Limburg bood hiertoe een unieke gelegenheid. Dat de nog aan te stellen kartrekker van deze Academische Werkplaats aan de promotie-eis diende te voldoen, was een voor de hand liggende voorwaarde. Dus stelde ik een plan op. Gerjo Kok, Nanne de Vries en Hans 


\section{DANKWOORD}

van Oers, thans mijn promotoren, hielpen mij over de eerste drempel van onzekerheid heen. Ook in de fasen erna bleven zij mij steunen. Nanne, bedankt voor je gedetailleerde commentaren en kritische noten, die je altijd positief wist in te kleden. Ik benijd het gemak waarmee je je werk lijkt te doen. Je beschikt bovendien over veel van iets, waar ik misschien te weinig van bezit: humor. Ook in andere opzichten kan ik nog veel van je leren. Gerjo, bedankt voor de helikopterblik waarmee je mijn concepten beoordeelde. Vooral op hoofdlijnen wist je sterk commentaar te leveren. Jij weet precies wat écht belangrijk is! Het uiteindelijke resultaat is er aanzienlijk door verbeterd. Hans, jij bedankt voor het meedenken, in de allereerste fase vooral, toen het idee nog vorm moest krijgen. Schuchter maakte ik je deelgenoot van mijn plannen. Je was meteen bereid om mee te denken over een meer concrete invulling van de op dat moment nog tamelijk vage contouren. Je optimistische kijk leidde ertoe dat ik zelf goesting bleef houden, ondanks de onvermijdelijke hobbels. Beste Nanne, Gerjo en Hans, als drie wijze heren hebben jullie mij geweldig terzijde gestaan. Ik voelde me bij jullie thuis, en dat is het beste wat je als promovenda kan overkomen.

Het karwei moest in twee jaar geklaard zijn, vond ik. Het schrijven van het proefschrift werd daarmee een individuele race tegen de klok, vergelijkbaar met een klimtijdrit tegen de flanken van de Mont Ventoux. Niet dat ik totaal onvoorbereid aan beide exercities begon. Eerder had ik reeds enkele tochten gefietst. Meestal ging ik onbevangen van start, zonder al te ver vooruit te kijken. Zo ook zijn de artikelen die als fundament voor dit proefschrift fungeren tot stand gekomen: in een eerder stadium, zonder het doel van een promotie voor ogen.

Een aantal jaren geleden fietste ik, daartoe aangespoord door Riekie, in Frankrijk de eendaagse variant van de Ardéchoise, een tocht over de uitlopers van de Franse Alpen. In totaal fietste ik die dag $166 \mathrm{~km}$. Tegen alle verwachtingen in werd ik met zilver bekroond. Later fietste ik de 13 kilometer lange klim naar Alpe d'Huez, met zijn 21 haarspeldbochten. In een vloek en een zucht, zo leek het, was ik boven, in een tijd van 1 uur en 9 minuten. Het stevige tempo en het ogenschijnlijke gemak waarmee ik het artikel over probleemjongeren heb geschreven, roepen herinneringen op aan deze klim. Riekie, jij was de initiator van mijn racefietscarrière, en tevens rolmodel voor het durven aangaan van nieuwe uitdagingen. Riekie, bedankt hiervoor! Door het fietsen heb ik geleerd wat doorzetten betekent, vooral op momenten dat je er helemaal door lijkt te zitten, 'uitgewoond' bent, om met wijlen De Kneet te spreken. De dood of de gladiolen! Sinds je vertrek uit Maastricht mis ik onze gezamenlijke tochten natuurlijk, en met weemoed denk ik terug aan de ideeën die geboren en de ervaringen die uitgewisseld werden, op de fiets.

Ook fietste ik ooit de Shimano Challenge. Een rit van $100 \mathrm{~km}$ voor 'liefhebbers', dwars door de Voerstreek, met 11 fikse hellingen. Dat was eens maar nooit weer. Waarlijk een barre tocht. Het was begin mei, maar het kwik bleef 
die dag steken bij 4 graden Celsius, en de regen kwam met bakken uit de hemel. Menige fietser werd met bevriezingsverschijnselen afgevoerd. Ik ontsprong maar net de dans, en werd uiteindelijk tweede in mijn categorie. Deze memorabele Shimano Challenge vertoont gelijkenis met de route waarlangs de artikelen over hepatitis B en sexueel overdraagbare aandoeningen tot stand zijn gekomen. Dankzij Harm hield ik het vol. Afstappen is mijn eer te na, maar het scheelde niet veel of ik had de pijp aan Maarten gegeven. Harm, bedankt voor je voortdurende stimulans om het artikel over hepatitis B af te maken. Het moest tussen alle bedrijven door gebeuren, en net als ik er weer in zat vroegen andere, dringender verplichtingen om voorrang. Jij had daar begrip voor, toonde geduld. Los van dit artikel ben ik ontzettend blij dat ik met jou heb mogen samenwerken. Reeds aan het begin van mijn carrière bij de GGD hadden we een gezamenlijk project over verkeersveiligheid bij ouderen. Wat hebben we gelachen! En daarna is dat altijd zo doorgegaan.

Verder heb ik regelmatig de Mergelheuvelland Tweedaagse gefietst. Eén keer, toen de Keutenberg beklommen moest worden nadat we al $98 \mathrm{~km}$ en 10 steile beklimmingen erop hadden zitten, beging ik een zonde. Ik kon niet meer en moest even, hooguit een minuut, van mijn fiets af. De man met de hamer, afgesneden benen, parcheggio. In deze - onvermijdelijke - zonde zie ik een parallel met het onderzoek naar het effect van verlengde openingstijden van gokhallen. Om pragmatische reden stemden we destijds in met een bij nader inzien dubieuze onderzoeksopzet, waarmee we feitelijk geen afdoende bewijs konden leveren voor het effect van de verlengde openingsduur. Ronald, ondanks alle methodologische beperkingen stimuleerde jij me met creatieve ideeën om toch een wetenschappelijk verantwoord artikel te schrijven over dit onderwerp. Je hebt me enorm geholpen bij deze klus. Onze discussies hebben me aangezet tot grotere prestaties. Ronald, bedankt!

Tussendoor rende ik nog tweemaal voor mijn plezier de $5 \mathrm{~km}$ loop van Maastrichts Mooiste. Kort maar krachtig. Achteloos werd ik 1e, respectievelijk $3 e$ in mijn klasse. Ik fietste de Petit Ballon en de Grand Ballon in de Vogezen. Eveneens korte, maar tevens zeer steile klimmetjes. Maar toch niet zulke kuitenbijters als de Gulpenerberg, dicht bij huis, die ik een tijdlang wekelijks 'deed'. Rechtdoor naar school en kantoor! Ik was dus wat gewend. Dankzij Mariken werd voor mij de totstandkoming van het Schoolslag-artikel een kort, maar krachtig traject. De voorbereidingen hadden reeds in een eerder stadium plaatsgevonden. We kwamen goed getraind aan de start. Mariken, jou wil ik bedanken voor je collegiale steun. Wij vormden een koppel in een américain en deelden de ervaringen van werkdruk, gezinszorgen en inspanningen om jezelf in balans te houden. Jouw tempo was mij soms toch te snel, dan moest ik lossen. Dat ik hier nu eerder sta dan jij heeft niets met tanende tempohardheid bij jou te maken, laat dat helder wezen. Ik kan je nog steeds niet bijbenen en dat wil wat zeggen. Ik hoop dat jij jouw boekje ook spoedig kunt afronden. 


\section{DANKWOORD}

In jaren dat het zo uitkwam fietste ik ook Limburgs Mooiste. Meestal koos ik voor de $100 \mathrm{~km}$. Nu is het met fietstochten zo dat een succesvolle afloop het bereiken van de finish - mede wordt bepaald door de afstand waarop je je vooraf instelt. Als je voor $100 \mathrm{~km}$ gaat, zul je dat ook wel halen. Maar als die afstand onverwachts tot $131 \mathrm{~km}$ oploopt, omdat de bewegwijzering niet klopt, wordt het plots een hele toer om aan de meet te geraken. Natuurlijk speelt mee dat je in zo'n geval vaak onvoldoende eten en drinken bij je hebt en ligt de hongerklop op de loer. Maar de haalbaarheid van een bepaalde afstand zit toch ook 'tussen je oren'. Dit fenomeen deed zich voor bij het schrijven van het Hartslag-artikel. Ik meende dat vrij snel klaar te kunnen hebben, maar de inhoud moest bij nader inzien anders. Kortom, het parcours pakte heel anders uit dan gepland. Janneke, jou wil ik in dit verband speciaal noemen. Een betere fietsmaat dan jij had ik niet kunnen treffen. Je dacht met me mee over de opzet en keer op keer becommentarieerde je zeer nauwgezet mijn concepten. Jouw inbreng heb ik zeer gewaardeerd. Dat ik je misschien soms onder tijdsdruk heb gezet spijt mij oprecht en was nooit de bedoeling.

Tot slot wachtte de Mont Ventoux. De Mont Ventoux is een berg in het departement Vaucluse in Zuid-Frankrijk. Het is de enige serieuze berg in de streek de Provence, die daarom ook wel de Reus van de Provence wordt genoemd. De naam van de berg is afgeleid van het Franse woord vent, dat wind betekent. Op de top waait het vaak erg hard, door de mistral. Er zijn windsnelheden tot $300 \mathrm{~km}$ per uur geregistreerd. De top ligt op 1912 meter hoogte. Het hoogteverschil met de voet van de berg bedraagt 1614 meter. Vooral de beklimming vanuit Bedoin is erg zwaar, door de lengte van de $\mathrm{klim}(21 \mathrm{~km})$, het stijgingspercentage (gemiddeld 7,6\%, met regelmatige uitschieters tot $11 \%$ ), de zomerse temperaturen in Zuid-Frankrijk, en het onherbergzame maanlandschap na het passeren van Chalet Reynard. Tijdens de zomervakantie van 2005, kort nadat ik m'n promotietraject had ingezet en luttele maanden voor mijn 50e verjaardag, was het dan zover. Min of meer bij toeval zetten we onze tenten op nabij Petrarca's Fontaine de Vaucluse, op fietsafstand van de Mont Ventoux. De vélo's stonden op de trekhaak, de mistral hield zich nog even gedeisd, en dus moest het er maar van komen. Natuurlijk kozen Martien en ik voor de weg van de meeste weerstand: de route vanuit Bedoin.

Gelukkig kreeg ik veel bijstand. Om te beginnen stemde de GGD-directie ermee in dat ik mijn stuwmeer van verlofdagen opnam. Fons, jou met name wil ik bedanken voor het behartigen van mijn belang in deze. Zonder die drie maanden zouden het raamwerk en de basistekst van het proefschrift nu nog lang niet zijn klaar geweest.

Monique zorgde ervoor dat mijn fiets geprepareerd werd: de bandjes werden opgepompt tot 7 bar atmosferische druk, de ketting voorzien van voldoende olie, de voorraad fietsbidons met water gevuld, reserve tubes in het fietstasje, remmen en versnelling gecontroleerd, loshangend stuurlint vastgeplakt en toe- 
clips ontdaan van modderresten. Tijdens de rit zorgde jij ervoor dat ik een tempo onderhield dat ik lang kon volhouden. Monique, jouw assistentie was goud waard! Als ik met grote haast kwam binnenrennen en iets uitgetypt moest hebben was jij steevast de reddende engel. Jij typte vele pagina's voor me uit en terwijl je dat deed redigeerde je de tekst en dacht je geïnteresseerd mee. Je warme karakter en je inlevingssvermogen waren een geweldige compensatie voor de stress die het proefschrift in wording op gezette tijden met zich meebracht.

Het begin ging in een aardig tempo. Eerlijkheidshalve moet ik bekennen dat de eerste vijf kilometer van de klim meer weg hadden van vals plat. Na dit stuk 'plakkende weg' werd het stijgingspercentage allengs groter. Floor bleef mij in elke bocht voorzien van gezoet water en veel bananen, onmisbare energiebronnen tijdens zo'n klim. Floor, bedankt voor je niet aflatende steun en belangstelling, zowel voor de inhoud van het proefschrift als voor de privéomstandigheden. Onze telefoongesprekken op de zaterdagavond, soms tot na middernacht, waren voor mij zowel een bron van inspiratie als een morele steun.

Mayke, jij stond langs dit parcours regelmatig en vaak geheel onverwacht met een verse bidon klaar, terwijl het zweet van mijn gezicht droop. Water, levensreddend water was het enige waar ik op die momenten naar snakte. Beste Mayke, met een nuchtere verwijzing naar de steun en ontspanning die jij me bood zou ik je tekort doen: we hebben de afgelopen 20 jaar samen heel wat afgekletst en -gelachen. En dat blijven we doen, lijkt mij!

De klim vorderde gestaag. Ik passeerde de boomgrens. De bossen maakten plaats voor kale rotsen. Daarom wordt de Mont Ventoux ook wel 'de kale berg' genoemd. Het werd steeds spannender of het wel zou lukken. Ik naderde het gedenkteken voor Tom Simpson. Hij heeft destijds het loodje gelegd. Aangezien ik een te groot verzet moest wegtrappen - ik heb een oude racefiets zonder triple en flappers - moest ik op de macht naar boven. Van moulineren was geen sprake meer. Stilaan voelde ik mijn kniegewrichten pijnlijker worden. Ik moest al mijn duivels ontbinden. Gelukkig stond op 5 kilometer vóór de finish een heel peloton supporters mij aan te moedigen en te adviseren over de laatste loodjes, juist op het moment dat ik bijna van mijn fiets werd gezwiept door de aanzwellende rukwinden. De $300 \mathrm{~km}$ per uur werd weliswaar niet gehaald, maar voor iemand in mijn gewichtsklasse kunnen aanzienlijk lagere windsnelheden al rampzalige gevolgen hebben. Ik wil op deze plaats het team van experts van harte bedanken. Jolanda, Jacomine, Hans, Theo, Fons, Anja, Willy, Nanne, Jacques, Roger, Evelien, Jeu, Hilde, Marleen, Lea, Carola, Kim en Angelique: bedankt! Ik heb het geweldig gevonden dat jullie allen voor mij speciaal naar de Mont Ventoux zijn afgereisd om mij met raad en daad bij te staan. Dank jullie wel!

Gesterkt door deze aanmoedigingen wist ik nu dat ik het zou gaan halen, ondanks de wind en de pijnlijke gewrichten. Ik fietste met een snelheid van nog 


\section{DANKWOORD}

maar amper $7 \mathrm{~km} /$ uur. Goed om om te vallen. Er leek maar geen einde aan te komen. In de verte ontwaarde ik Sophie, Désirée en Wouter met een spandoek: "Hup Maria, hup. Bridge the gap". Zij ontwierpen de omslag van dit proefschrift. Ondanks hun drukke onderwijsprogramma gingen zij vele malen naar tekenles bij Marijke. Marijke, jouw inzet, zorgvuldigheid, invoelingsvermogen en warme uitstraling hebben ertoe bijgedragen dat dit kaftontwerp met heel veel plezier en bezieling gemaakt is. Sophie, Désirée en Wouter, ik vind het geweldig wat jullie hebben gemaakt! Dit proefschrift kan met recht een gezinswerkstuk genoemd worden. De symboliek druipt ervan af. Naar de aard van het beestje vertegenwoordigt Wouter het beleid, Désirée de praktijk en Sophie het onderzoek. Het kan natuurlijk ook best een slag anders zijn. Hoe dan ook, jullie kunnen het heel goed vinden met elkaar! Dat jullie tot in lengte van jaren op dezelfde voet mogen blijven samenwerken.

Met een gemiddelde van $10 \mathrm{~km} /$ uur passeerde ik na 2 uur en 9 minuten de finish. Martien fungeerde gedurende de hele tocht als maatje en als steun in de rug. Martien, je kent dat wel, het gebruikelijke duwtje als ik er even 'doorzit'. De eerste kilometers leidde jij nog de dans en schudde aan de boom, daarna hing je aan de rekker, speelde accordeon, en kreeg je een patat. Piano, piano! Uiteindelijk kwam jij 8 minuten na mij binnen. Te weinig kilometers in de benen en te veel -grammen elders. Naar eigen zeggen arriveerde je op reserve, uit angst jezelf op te blazen, met twee vingers in de neus (als je niet beide handen aan het stuur nodig had gehad om niet van de weg geblazen te worden), dat weer wel. Ik heb weliswaar de tijdrit en de bolletjestrui voor de snelste klimmer gewonnen, maar zonder jou was dat niet gelukt! Ik ben intussen tot programmaleider van de Academische Werkplaats benoemd. Tot het moment van de openbare verdediging en de uitspraak van de voorzitter van de corona ben ik inderdaad als enige van de Academische Werkplaatsleiders in ons land nog niet in het bezit van de doctorstitel. Of ik de eindstreep definitief ga halen zal op 28 juni blijken. Eerst zien, dan geloven.

Bij de finish wachtte de fanclub van supporters me op. Yvonne, zonder jouw assistentie bij de huishoudelijke taken had ik deze tocht nooit kunnen uitrijden. Na 15 jaar trouwe dienst hoor je gewoon bij ons. Duizendmaal dank voor al je inzet! Marguérite, met jouw verrassingspakketten bij geboorten, verjaardagen en andere feestmomenten, of gewoon zomaar, raakte je vaak een gevoelige snaar bij mij. Ik verbaas me nog steeds over jouw creatieve momenten. Dank voor al je hartelijkheid en de geweldige steun op momenten waarop wij dat nodig hadden. Verder wil ik Birgitte, Ruud, Loek, Nicole, Han en alle andere UM-sportdocenten bedanken. Sporten is mijn uitlaatklep. Het verstand gaat dan even op nul en de blik op oneindig. Het lunchpauzeprogramma waarin we kunnen skippen, spinnen of conditietrainen, helpt mij bij het relativeren van het belang van het werk en van de werkdruk. Dank dus aan het UMsportdocententeam. Erik, bedankt voor de inspirerende gesprekken die we 
samen hebben gevoerd. Je hebt me enorm aangemoedigd! Jan, jij bedankt voor het redigeren van mijn steenkolen engels. Qua leesbaarheid is het boekje er dankzij jou aanzienlijk beter op geworden. Ook wil ik graag mijn allerbeste vrienden en mijn naaste familie bedanken, die allemaal op hun eigen manier hebben gezorgd voor de broodnodige ontspanning. Marga, Peter, Ina, Ton, Riekie, Floor en Rob, reuze bedankt voor de gezelligheid die we jaar in jaar uit in de Ardennen beleven. Vader ( $\dagger$ ), Moeder, Rieneke, Harrie, Jan, Nel, Piet, Ivonne, Frank, Edith, Marianne, en aanhang, en natuurlijk ook schoonvader, moeder(†) en -familie: bedankt voor jullie betrokkenheid! Tot slot dank ik de leden van de beoordelingscommissie voor hun werk. Ik realiseer me dat het een hele klus is om een proefschrift als dit te beoordelen. Ik heb serieus geprobeerd het 'boekje' binnen de 200 pagina's te houden, maar door het opnemen van zes artikelen is me dat niet gelukt. Sorry!

De beklimming zit erop, alle etappes zijn verreden. Iedere etappe was zwaarder dan de vorige. Daarom nu eerst even recupereren. 


\section{Curriculum vitae}

Maria Jansen werd geboren op 26 januari 1956 in Druten, Gelderland. In 1974 behaalde zij haar Atheneum B diploma aan het Pax Christi College te Druten. In hetzelfde jaar startte zij met de studie Humane Voeding aan de Landbouw Universiteit Wageningen. Deze opleiding sloot zij in september 1981 af, met als doctoraal vakken Voedingsleer, Gezondheidsleeer en Sociologische aspecten van de ontwikkelingsplanning in niet-westerse gebieden. Tevens behaalde zij het bewijs van voldoende pedagogisch-didactische scholing. Van oktober 1981 tot april 1989 was Maria werkzaam als docent. Zij doceerde voedingsleer en gezondheidskunde aan de Hogere Beroepsopleiding Diëtetiek te Heerlen en daarna patiëntenvoorlichting en gezondheidszorgonderzoek aan de Hogere Beroepsopleiding voor Verpleegkunde te Amsterdam. In april 1989 werd Maria aangesteld als gezondheidsvoorlichtster bij de GGD Zuidelijk Zuid-Limburg (thans GGD Zuid Limburg), waar zij in samenwerking met de Universiteit Maastricht onderzoek deed naar de verkeersveilige mobiliteit van 55-plussers. Hierna werd zij coördinator verslavingszorg en aidspreventie. In deze functie initieerde zij diverse activiteiten, zoals de spuitenomruil voor drugsverslaafden, aidspreventieprogramma's voor homo- en biseksuelen, een geslachtsziektenpoli met aandacht voor preventie, bijstelling van het lokale gokautomatenbeleid, surveillance van infectieziekten onder risicogroepen en preventieprogramma's voor (risico)jeugdigen gericht op gezond gedrag. Als praktijkprofessional kreeg zij de mogelijkheid om multidisciplinaire en intersectorale samenwerking aan te moedigen. In 1996 werd zij clustercoördinator bij de afdeling Algemene Gezondheidszorg van de GGD, waar zij een team van 25 medewerkers leidde. Vanuit deze positie wist zij de samenwerking met gemeenten, Universiteit Maastricht, Academisch Ziekenhuis Maastricht en diverse uitvoeringsinstanties te intensiveren. In 2000 werd zij benoemd tot senior beleidsmedewerker innovatie en adviseerde zij de directie ten aanzien van innovatieve trajecten binnen de openbare gezondheidszorg. In deze rol initieerde zij schoolSlag, Hartslag 2, Bewegen Zonder Zorgen (om spanningsklachten bij vrouwen in achterstandsbuurten te verminderen) en de Academische Werkplaats voor Publieke Gezondheid Limburg. Sinds augustus 2006 bekleedt zij de functie van programmaleider van deze Academische Werkplaats, een samenwerking tussen gemeenten, GGD, Universiteit Maastricht en Academisch Ziekenhuis Maastricht en geassocieerde partners waaronder de GGD Noord en Midden Limburg en de Thuiszorgorganisaties voor de 0-19 jarige jeugdgezondheidszorg.

Maria heeft het geluk met Martien van Dongen getrouwd te zijn en moeder te zijn van drie kinderen: Wouter (1987), Désirée (1990) en Sophie (1990). 
